\title{
Deficiency of MMP17/MT4-MMP Proteolytic Activity Predisposes to Aortic Aneurysm in Mice
}

Mara Martín-Alonso ${ }^{1}$, Ana B. García-Redondo ${ }^{2}$, Dongchuan Guo ${ }^{3}$, Emilio Camafeita ${ }^{4}$, Fernando Martínez ${ }^{5}$, Arántzazu Alfranca ${ }^{1}$, Nerea Méndez-Barbero ${ }^{1}$, Ángela Pollán ${ }^{1}$, Cristina Sánchez-Camacho ${ }^{6}$, David T.

Denhardt $^{7}$, Motoharu Seiki ${ }^{8}$, Jesús Vázquez ${ }^{1,4}$, Mercedes Salaices ${ }^{2}$, Juan Miguel Redondo ${ }^{1}$, Dianna Milewicz ${ }^{3}$ and Alicia G. Arroyo ${ }^{1}$

${ }^{1}$ Vascular Biology and Inflammation, Centro Nacional de Investigaciones Cardiovasculares Carlos III (CNIC), 28029 Madrid Spain; ${ }^{2}$ Department of Pharmacology/Nephrology, Faculty of Medicine, Universidad Autónoma de Madrid, 28029 Madrid, Spain; ${ }^{3}$ Department of Internal Medicine, University of Texas Health Science Center at Houston, Houston, 77030 Texas, USA; ${ }^{4}$ Proteomics Unit; ${ }^{5}$ Bioinformatics Unit, Centro

Nacional de Investigaciones Cardiovasculares Carlos III (CNIC), 28029 Madrid Spain; ${ }^{6}$ Universidad

Europea de Madrid, Villaviciosa de Odón, 28670 Madrid, Spain; ${ }^{7}$ Department of Cell Biology and Neuroscience, Rutgers University, Piscataway, New Jersey, USA, and; ${ }^{8}$ Division of Cancer Cell Research, Institute of Medical Science, University of Tokyo, Minato-ku, 108-8639 Tokyo, Japan.

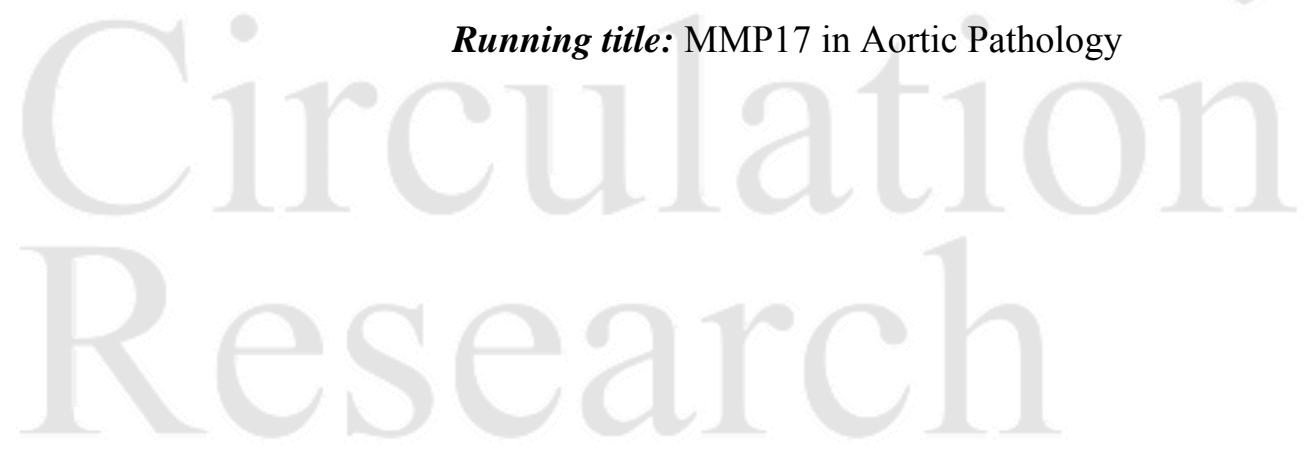

\section{Subject codes:}

[162] Smooth muscle proliferation and differentiation

[130] Animal models of human disease

\section{Address correspondence to:}

Dr. Alicia G. Arroyo

Matrix Metalloproteinases Lab

Centro Nacional de Investigaciones Cardiovasculares (CNIC)

Melchor Fernández Almagro 3

28029 Madrid

Spain

Tel 34914531241 ext 1159

Fax 34914531265

agarroyo@cnic.es

In April 2015, the average time from submission to first decision for all original research papers submitted to Circulation Research was 13.84 days. 


\section{ABSTRACT}

Rationale: Aortic dissection or rupture resulting from aneurysm causes $1-2 \%$ of deaths in developed countries. These disorders are associated with mutations in genes that impact vascular smooth muscle cell (VSMC) differentiation and contractility or extracellular matrix (ECM) composition and assembly. However, up to $75 \%$ of patients with a family history of aortic aneurysms do not have an identified genetic syndrome.

Objective: To determine the role of the protease MMP17/MT4-MMP in the arterial wall and its possible relevance in human aortic pathology.

Methods and Results: Screening of patients with inherited thoracic aortic aneurysm and dissections (TAAD) identified a missense mutation $(\mathrm{R} 373 \mathrm{H})$ in the $M M P 17$ gene that prevented expression of the protease in human transfected cells. Using a loss-of-function genetic mouse model, we demonstrated that the lack of Mmp17 resulted in the presence of dysfunctional VSMCs and altered ECM in the vessel wall; and it led to increased susceptibility to angiotensin II-induced thoracic aortic aneurysm (TAA). We also showed that Mmp17mediated Opn cleavage regulated VSMC maturation via JNK signaling during aorta wall development. Some features of the arterial phenotype were prevented by reexpression of catalytically active Mmp17 or the Nterminal osteopontin (Opn) fragment in Mmp17-null neonates.

Conclusions: Mmp17 proteolytic activity regulates VSMC phenotype in the arterial vessel wall, and its absence predisposes to thoracic aortic aneurysm in mice. The rescue of part of the vessel wall phenotype by a lentiviral strategy opens avenues for therapeutic intervention in these life-threatening disorders.

\section{Keywords:}

MMP17, aorta, cardiovascular diseases, aneurysms, osteopontin, matrix metalloproteinases. 
Nonstandard Abbreviations and Acronyms:

AAA

abdominal aortic aneurysm

COMP

ECM

cartilage oligomeric matrix protein

ERK

FBN1

GO

GPI

HEK

IRES

iTRAQ

JNK

Ki67

extracellular matrix

LDS

LV

MFS

extracellular signal-regulated kinases

fibrillin-1

gene ontology

glycosylphosphatidylinositol

human embryonic kidney

internal ribosome entry site

isobaric tag for relative and absolute quantification

c-Jun N-terminal kinase

protein encoded by MKI67 gene

Loeys-Dietz syndrome

lentivirus

MMP

Marfan syndrome

MT-MMP

matrix metalloproteinase

mTOR

OPN

PDGF

$\mathrm{pH} 3$

Q-RT-PCR

RGD

membrane-type MMP

mammalian target of rapamycin

osteopontin

platelet-derived growth factor

phosphorylated histone $\mathrm{H} 3$

quantitative-reverse transcriptase PCR

SFFV

SILAC

SM-MHC

TAAD

TEM

arginine (R), glycine (G), aspartic acid (D)

spleen focus-forming virus

stable isotope labeling of amino acids in culture

smooth muscle myosin heavy chain

thoracic aortic aneurysms and dissections

transmission electron microscopy

TGF

TIMP

THBS

VSMC

transforming growth factor

tissue Inhibitor of metalloproteinase

thrombospondin

WES

vascular smooth muscle cell

whole exome sequencing 


\section{INTRODUCTION}

Aortic aneurysm predisposes the aorta to dissection or rupture, and causes $1-2 \%$ of deaths in developed countries. ${ }^{1}$ Thoracic aortic aneurysms and acute aortic dissections (TAADs) that affect young patients result primarily from genetic mutations. ${ }^{2}$ The vulnerability of the aortic wall is mainly due to its poor ability to appropriately accommodate hemodynamic changes. The aortic wall, particularly in the thoracic aorta, is able to distend without requiring subsequent contraction of vascular smooth muscle cells (VSMCs) because of the elastic recoil provided by the layers of elastin lamellae. ${ }^{3}$ These mechanical properties depend on the intimate physical and mechanical connections of differentiated VSMCs with the extracellular matrix (ECM, mostly elastin and collagen) that they produce and organize, connections that are established through forcedependent adhesion proteins (integrins) and the cytoskeleton (actin and myosin). ${ }^{3}$ During aortic development, VSMCs differentiate from neural crest and second heart field progenitors, and also from mesenchymal progenitors recruited to the nascent endothelial tube; during this process proper interaction needs to be established between VSMCs and the ECM. ${ }^{4}$ Detailed analysis of pulmonary artery development demonstrated that formation of the vessel wall involves the induction of successive layers of VSMCs and the invasion of the outer layers through the reorientation and radial migration of the inner ones. ${ }^{5}$ Transforming growth factor (TGF) B has been extensively studied as an inducer of VSMC maturation, and more recent studies have identified gradients of platelet-derived growth factor (PDGF) B and other not-yet identified signals as regulators of VSMC differentiation, providing new insights into artery wall development. ${ }^{5}$

This intimate connection between VSMCs and the ECM ensures that alterations to one impact the other, and can have similar effects on the overall mechanical behavior of the vessel. Thus TAADs can be caused by mutations that affect VSMC function or that alter ECM composition and assembly. ${ }^{2}$ For example Marfan syndrome (MFS), a genetic syndrome that predisposes to TAAD, is caused by mutations in the fibrillin1 (FBN1) gene, which encodes an ECM protein necessary for proper elastin formation, ${ }^{6}$ whereas in LoeysDietz syndrome (LDS) TGFB receptors type 1 and 2 (TGFBR1 and TGFBR2) are mutated, ${ }^{7}$ resulting in impeded VSMC differentiation. ${ }^{8}$ Predisposition to TAAD is also conferred by mutations predicted to disrupt the ability of the VSMC to contract. Nonetheless, as many as $75 \%$ of patients with a family history of aortic aneurysms do not have an identified genetic syndrome. ${ }^{9}$ Recent efforts to identify novel cellular processes and molecular pathways relevant to these disorders have focused on the application of large-scale omics technologies in mouse models of aortic disease. The availability of whole exome sequence (WES) data allows the genes identified in these analyses to be assessed for mutations in patients with inherited TAAD., ${ }^{910}$

Matrix metalloproteinases (MMPs), part of the metzincin family of proteases, are able to cleave all ECM components and can also process transmembrane receptors and matrix-bound soluble factors. ${ }^{11}$ MMPs are proposed to actively participate in vessel wall remodeling, ${ }^{12}$ either through direct degradation of elastin, for example by the elastase Mmp12, or by promoting dedifferentiation and activation of VSMC, for example by Mmp14 (Mt1-mmp). ${ }^{13-15}$ Several MMPs are expressed in mouse embryonic aorta; ${ }^{16}$ however, their potential role in arterial vessel wall development and function has not been addressed.

Here we report that Mmp17 (also called Mt4-mmp, membrane-type 4-matrix metalloproteinase), is essential for VSMC maturation and function in the arterial vessel wall. Mmp17, which anchors to the plasma membrane through a glycosylphosphatidylinositol (GPI) link, is expressed in adult aortic VSMCs ${ }^{17}$ but its substrates and function are poorly characterized..$^{18}$ By genomic analysis we have identified a missense mutation in MMP17 in a patient with TAAD. A genetic model of Mmp17 loss-of-function in mice demonstrated the requirement of Mmp17 activity for VSMC maturation and arterial wall function in vivo and predisposition to thoracic aortic aneurysm in its absence. We also show that osteopontin (Opn) is a required Mmp17 substrate during embryonic development of the aorta. Expression of active Mmp17 or the N-terminal Opn fragment rescued several phenotypic features in Mmp17-null aortas suggesting avenues for therapeutic intervention in TAADs. 


\title{
METHODS
}

\begin{abstract}
Mouse line.
Mmp17 $7^{-/}$mice are in the C57BL/6 background. ${ }^{17}$ Mice were handled under pathogen-free conditions in accordance with CNIC institutional guidelines. Experiments were performed in accordance with Spanish legislation on animal protection and were approved by the local governmental animal care committee. Experiments were conducted on mice in three age groups: embryos (E10.5 to E18.5), neonates (P0 to P30), and adults (8-to-16 weeks old).
\end{abstract}

A detailed methods section is provided in the Online Data Supplement.

\section{RESULTS}

Genetic analysis of patients affected by TAAD identified an MMP17 missense mutation.

Previous analysis of the relationship of MMP17 mRNA levels with aortic aneurysms and other

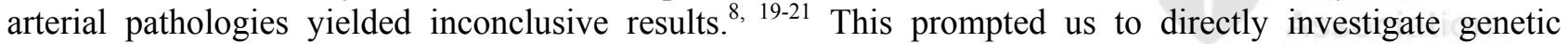
mutations in MMP17 in individuals with acute aortic dissection involving the ascending or descending aorta. We performed WES in samples from 58 probands from families with an inherited predisposition to TAAD. This analysis identified the missense mutation R373H (p.Arg373His) in the MMP17 gene (Figure 1A) of a European American male who had a type A aortic dissection (ascending aorta) at the age of 43 years with an aortic root diameter of $5.5 \mathrm{~cm}$ and who also suffered a stable chronic type B dissection (non-ascending aorta). Unfortunately no DNA samples were available from the proband's family to determine segregation of the R373H variant with disease. However, two out of three of the proband's siblings developed ascending aortic aneurysm and his father had a type A dissection. Polyphen analysis indicated that the identified mutation was likely to be deleterious, and in silico modeling predicted that the R373H mutation in human MMP17 would cause a conformational change in the C-terminal region (Figure 1B); such a change would presumably impede GPI-tail binding by transamidases during synthesis of the MMP17 variant on the endoplasmic reticulum, and therefore likely compromise its expression at the plasma membrane. Because MMP17 forms functional dimers at the plasma membrane, ${ }^{22}$ we modeled the MMP17 homodimer conformation in silico, finding that the R373H mutation might also affect the arrangement of the catalytic center of the homodimer (Figure 1B). In silico modeling of a heterodimer formed by wild-type MMP17 and R373H mutant monomers yielded no energetically stable conformation, suggesting that the mutation in heterozygosity might behave as a functional homozygous loss.

To test the impact of the R373H mutation on MMP17 expression, we transfected human embryonic kidney (HEK) 293 cells with wild-type or R373H mutant MMP17. Levels of MMP17 mRNA were similar in wild-type- and R373H-transfected HEK293 cells (Figure 1C), but the mutant MMP17 protein was undetectable by western-blot or immunostaining (Figure 1D and 1E). Mutant protein was, however, observed in intracellular vesicles upon treatment of R373H-MMP17-transfected HEK293 cells with the proteasome inhibitor bortezomib (Figure 1E), suggesting that the mutant MMP17 gets degraded during its synthesis in the endoplasmic reticulum.

\section{Mmp17-deficient mice are more susceptible to thoracic aortic aneurysm.}

These data suggested the possibility that the MMP17 mutation might predispose to aortic pathology; however since no DNA samples were available from the family to determine segregation of the R373H variant with disease and the co-existence of other pathogenic gene mutations was not thoroughly ruled out, we decided to directly analyze the impact of MMP17 absence on the arterial vasculature in vivo in a loss-of-function 
animal model, Mmp17 knock-out/lacZ knock-in mice $\left(M m p 17^{\text {lacZ/lacZ }}\right.$, referred to here as $\left.M m p 17^{-/ /}\right){ }^{17}$ After confirming the expression of Mmp17 in the smooth muscle cells of different arterial vascular beds of adult wild-type mice (Online Figure I) ${ }^{17}$ we explored the effect of its absence on arterial parameters in $M m p 17^{-/-}$ mice. Magnetic resonance imaging of live mice revealed that the diameter of the lumen of the descending aorta was $14 \%$ larger in $M m p 17^{-/}$mice $\left(1.076 \pm 0.026 \mathrm{~mm}\right.$ in wild-type versus $1.159 \pm 0.018 \mathrm{~mm}$ in $\mathrm{Mmp}^{-1 /-}$ ) (Figure 2A). The ascending aorta transverse area was slightly but not significantly larger in histological sections, as this was similarly observed by ultrasound analysis, which also revealed an altered movement in the ascending aortas of $M m p 17^{-/}$mice with respect to wild-types (Online Figure II; Online Movies I and II). Moreover, $\mathrm{Mmp} 17^{-/-}$mice became hypotensive 8 weeks after birth without showing differences in heart rate (Figure 2B).

Susceptibility to aneurysm in mice can be masked by hypotension; therefore to analyze this susceptibility we treated 16-week-old $M m p 17^{-/-}$and wild-type mice with angiotensin II and monitored blood pressure and aortic diameter in the aortic arch and the abdominal area. Angiotensin II increased blood pressure in both groups (unpublished data). Three out of $14 \mathrm{Mmp} 17^{-/-}$mice died by aortic rupture or dissection $(21.43 \%)$, whereas no deaths were registered in the wild-type group. Consistent with this finding, Mmp17-null mice developed TAA at a higher incidence than wild-types (42.86\% in Mmp17-null mice vs $7.14 \%$ in wild-type; $\mathrm{p}=0.0384$ by one-tailed Fisher's exact test); thoracic aortas in Mmp17-deficient mice were also significantly more dilated after 4 weeks treatment with angiotensin II (Figures $2 \mathrm{C}, 2 \mathrm{D}$, and $2 \mathrm{E}$ ). Although $M m p 17^{-/}$mice showed no overall increased incidence of abdominal aortic aneurysm (AAA) in response to angiotensin II (33.33\% in $\mathrm{Mmp}_{17^{-/}}$mice vs $28.54 \%$ in wild-type; $\mathrm{p}=0.5629$ by one-tailed Fisher's exact test), abdominal aortic diameter was significantly larger than in wild-types (Figures $2 \mathrm{~F}, 2 \mathrm{G}$ and $2 \mathrm{H}$ ).

We next analyzed the response of $M m p 17^{-/}$vasculature to another model of vascular injury, triggered by ligation of the carotid artery. The carotid vessel of Mmp17-null mice displayed outward remodeling, contrasting with inward remodeling in wild-type carotid arteries (Online Figure IIIA and IIIC). Moreover, after 4 weeks of ligation, neointima formation was more-prominent in $M m p 17^{-/}$carotid arteries, correlating with greater numbers of proliferative VSMCs (Online Figure IIIB and IIID). These data confirm the pathological response of Mmp17-null vasculature to injury.

\section{Mmp17 absence affects VSMC orientation and function.}

Transmission electron microscopy (TEM) of transverse and longitudinal sections of the aortic vessel wall revealed an aberrant phenotype in the VSMCs of Mmp17-null aortas in basal conditions (Figure 3A). ${ }^{23}$ These VSMCs were rounder, disconnected from the elastin lamellae, multilayered between elastin lamellae, and helically/radially oriented in contrast to wild-types (Figure 3A). Similar VSMC and ECM alterations were observed in the aortas of heterozygous mice (unpublished data).

These changes were accompanied by altered distributions of the VSMC markers calponin and smooth muscle myosin heavy chain (SM-MHC, also called Myh11) in Mmp17 ${ }^{-/}$aortas (Figure 3B), and the rounded morphology and increased size were confirmed in VSMCs freshly isolated from $M m p 17^{-/}$aortas (Online Figure IV). Histological analysis of vessel wall structure in the descending aortas of $M m p 17^{-/-}$mice also revealed higher density of VSMC nuclei than found in wild-type counterparts (Figure 3C), a finding confirmed by counting isolated aortic VSMCs (Figure 3D). However, the number of elastin lamellae was unaltered, and immunostaining of phosphorylated histone $\mathrm{H3}$ (pH3) or Ki67 (protein encoded by MKI67 gene) showed no proliferation in $M m p 17^{-/-}$adult aorta (unpublished data).

Mmp17 $17^{-/-}$aortas also showed more abundant fibrillar ECM deposits and frayed elastin lamellae (Figure 3A). Verhoeff-van Gieson and Alcian blue staining showed no major differences in the abundance of elastin or glucosaminoglycans (Online Figure VA). However, picrosirius red and second harmonic generation multi-photon microscopy revealed a greater abundance of collagen fibers in Mmp17-null aortas, particularly 
in the adventitia (Online Figure VB). Analysis of non-pressure-fixed aortas revealed a significantly thicker vessel wall in $M m p 17^{-/-}$mice that was not apparent in pressure-fixed vessels (Figure 3E), indicating a different vessel-wall structure and behavior in response to pressure, in line with the angiotensin II experiment. Consistently, Mmp17/- mesenteric arteries, which showed similar VSMC and ECM features in TEM analysis to those seen in aorta, underwent higher stress under pressure in ex vivo assays and contained a below-normal fenestra area in the internal elastic lamina, indicative of altered structure (Online Figure VI) ${ }^{24}$

\section{Early postnatal onset of the arterial phenotype in Mmp17-deficient mice.}

TEM analysis of Mmp17-null neonatal aortas revealed rounder and differently distributed VSMCs as early as postnatal day 0 (P0) in transverse and longitudinal sections (Figure 4A). Compared with stagematched wild-type VSMCs, Mmp17 ${ }^{-/-}$VSMCs contained fewer contractile filaments, more rough endoplasmic reticulum and were disconnected from the elastin lamellae (Figure 4A). Moreover, immunostaining for $\mathrm{pH} 3$ revealed significantly more VSMCs proliferating in the aortic media layer in $M m p 17^{-/}$mice at P0 to P7 (Figures 4B and 4C). Analysis of Tgfb2 and Tgfb1, master regulators of VSMC differentiation, ${ }^{1,25}$ also revealed below normal levels of Tgfb2 mRNA in aorta extracts from $M m p 17^{-/-} \mathrm{P} 7$ mice, with no alteration in the levels of Tgfb1 mRNA (Figure 4D).

Consistent with the early (P0) phenotype of Mmp17-null mice, LacZ/ $\beta$-galactosidase expression (indicating Mmp17 promoter activation) in developing embryos was evident in peri-aortic VSMC progenitors of heterozygotes $\left(\mathrm{Mmp} \mathrm{7}^{+/ \mathrm{lacZ}}\right)$ at E10.5-11.5, with the highest number of $\beta$-galactosidase-positive VSMCs detected in the developing aortic media layer at E14.5. (Online Figure VII). Mmp17 promoter activation subsequently declined at later embryonic stages (E16.5 and E18.5, Online Figure VII).

Proteome analysis of the early and late aortic phenotype in Mmp17-deficient mice.

To understand the molecular mechanisms affected in the absence of Mmp17, we performed a multiplexed quantitative proteomics analysis of protein extracts from neonatal (P7) and adult (8-week-old) aortas obtained from Mmp17 $17^{--}$and wild-type mice. We determined the relative abundance between the null and wild-type mice of 2096 proteins in neonates and 2767 proteins in adults. Significant abundance differences between the two genotypes were found for 219 proteins in neonates and 207 proteins in adults. Functional category analysis (Online Table I) identified alterations related to improper VSMC maturation (increased ribonucleoprotein complex/protein synthesis and decreased muscle contraction and intermediate filaments) as the early event in neonatal Mmp17-null aortas (Figure 5A), whereas adult Mmp17-null aortas were characterized by an overrepresentation of categories related to ECM (in particular collagen) and cellular shape and adhesion (Figure 5B). Notably, neonatal and adult $M m p 17^{-/-}$aortas both had a below normal representation of the mitochondrion category.

\section{Lentivirus-based gene therapy rescues the arterial phenotype of Mmp17-null mice.}

We next tested whether the arterial phenotype of $M m p 17^{-/-}$mice could be rescued by lentivirus-driven restoration of Mmp17 function in VSMCs. Lentivirus encoding wild-type mouse Mmp17 (Figure 6A) was injected into $M m p 17^{-/-}$mice on postnatal day 1 (P1). After confirming lentivirus-derived Mmp17 protein expression and corresponding Mmp17 mRNA levels in aortic VSMCs 7 days after injection (Online Figure VIIIA and VIIIB), ${ }^{26}$ we analyzed early (P8) and late (8 weeks) phenotypic features (Figure 6B). Expression of catalytically active Mmp17 from P1 restored VSMC proliferation to normal levels at P8 (Figure 6C) and prevented aortic wall alterations in adult $M m p 17^{-/-}$mice, including diastolic pressure, density of VSMC nuclei, wall thickness in non-pressure-fixed aortas, VSMC morphology and orientation and ECM organization, and distribution of the contractility marker calponin (Figures 6D to 6H). No recovery of phenotypic features was observed in Mmp17-null aortas expressing lentivirus encoding the catalytically inactive E248A Mmp17 mutant, demonstrating that Mmp17 catalytic activity is necessary for its function in the vessel wall. 
To analyze the possible benefit of this strategy in already established disease, we injected lentivirus into adult mice (8-week-old) and analyzed aortas 8 weeks later. Reexpression of catalytically active Mmp17 in adult $\mathrm{Mmp} 17^{-/}$mice rescued some of the aortic features, in particular density of nuclei, while the inactive E248A Mmp17 had no effect (Online Figures VIIIC, VIIID and VIIIE and unpublished data).

\section{Mmp17-mediated cleavage of Opn during aorta development is required for c-Jun N-terminal kinase (JNK) signaling in VSMCs.}

MMP17 substrates are poorly characterized, with no vascular substrate proposed so far. The dependence of the vascular action of Mmp17 on its catalytic activity prompted us to conduct a SILAC (stable isotope labeling of amino acids in culture) screen for possible substrates in supernatants of cultured cells derived from wild-type or null mice. From a list of putative substrates including osteopontin (Opn), thrombospondin 4 (Thbs4) and cartilage oligomeric matrix protein (Comp), we selected Opn, a matricellular protein expressed in the vessel wall — including embryonic aorta - and associated with aortic aneurysm and VSMC migration and differentiation. ${ }^{27-30}$ We validated human MMP17-mediated OPN cleavage by in vitro digestion (Figure 7A), and N-terminal sequencing determined the cleavage site in human OPN to be between $\mathrm{Asp}^{210}$ and $\mathrm{Leu}^{211}$ (Figure 7B). We next modeled the possible apposition of OPN to dimeric MMP17 by in silico docking, obtaining a stable model of human OPN in the catalytic center of the MMP17 dimer (Figure 7C).

We next analyzed the putative relevance of Mmp17-mediated Opn cleavage in mouse aorta. Opn expression was detected by immunostaining in embryo aortas, with particularly strong expression at E14.5, correlating with the observed Mmp17 expression by lacZ and anti- $\beta$-galactosidase staining (Figure 7D; Online Figure VII). More intense Opn immunostaining was observed in Mmp17-null aortas at E14.5 (Figure 7D) and western-blot showed accumulation of full length Opn in E14.5 aorta extracts from Mmp17-null embryos with no changes in Opn mRNA levels (Figure 7E and 7F). Opn accumulation was also observed in E14.5 cartilage extracts, a tissue in which Mmp17 is also expressed, but not in aortas or cartilage from P7 Mmp17-null neonates (unpublished data). Moreover, the N-terminal Opn fragment was significantly less abundant in Mmp17-null E14.5 aortas (Figure 7E). This N-terminal Opn fragment generated by Mmp17 cleavage in the aorta at E14.5 would contain an active RGD (arginine (R), glycine (G), aspartic acid (D)) site for binding to $\alpha v \beta 3$ integrin, known to regulate VSMC adhesion and migration. ${ }^{31}$ Immunostaining analysis of several signaling pathways potentially triggered by this Opn fragment showed selectively fewer phospho-JNK-positive VSMCs in E14.5 Mmp17-null aortas (Figure 7G). These data suggest that regulated and timely cleavage of Opn by Mmp17 is required for proper activation of JNK signaling in the VSMCs of the developing aortic vessel wall.

To investigate the possible link between the reduction of Opn processing and the aortic wall phenotype of Mmp17-null mice, we generated lentiviruses encoding full length Opn and the predicted cleaved $\mathrm{N}$-terminal Opn fragment (Figure 8A) and injected them into P1 Mmp17-null neonates. Production and secretion of Opn protein forms was confirmed in vitro by infection of HEK293 and western-blot of culture supernatants (Online Figure IXA). On day 7 after lentivirus injection, GFP signal was detected in the aortas of neonatal $\mathrm{Mmp17^{-/ }}$ mice (Online Figure IXB). Expression of the N-terminal Opn fragment, but not full length Opn, in Mmp17-null mice restored normal proliferation of VSMCs (pH3-positive cells) in the aortic vessel wall 7 days after injection (Figures 8B and 8C). Immunostaining of aortas from neonate null mice injected with the N-terminal Opn fragment lentivirus also showed significantly increased phospho-JNK in the arterial media (Figure 8D and 8E), indicating that the N-terminal Opn fragment is required for proper activation of JNK signaling in the vessel wall. Finally, as shown in Online Figure IXC, overexpression of the N-terminal Opn fragment rescued the area and roundness in cultured Mmp17-null aortic VSMC, reinforcing the role of N-terminal Opn-mediated signaling in determining a normal VSMC phenotype. 


\section{DISCUSSION}

Despite the identification and characterization of several mutations responsible for familial TAAD, as many as $75 \%$ of inherited syndromes have no known origin. Although MMP17 mRNA levels have been analyzed in tissue samples and VSMCs from patients with aortic aneurysms and other arterial pathologies, these previous studies yielded inconsistent results regarding a pathogenic effect of altered MMP17 levels in these disorders. ${ }^{8,19-21}$ Our exome sequencing data point to missense mutations in human MMP17 gene as a predisposing factor for some of these cases; however, larger population samples, segregation of the mutation with the disease, and coexistence with other mutations should be analyzed in order to estimate the pathogenic relevance of mutations in this gene and the percentage of TAAD patients affected by them.

In this study we demonstrate the causative role of Mmp17 in aortic pathology through the use of a loss-of-function genetic mouse model. Mmp17-null mice had dilated aortas, with VSMCs rendered dysfunctional by altered morphology and distribution and associated changes to the ECM. These animals were hypotensive and displayed an adaptive adventitial fibrosis, as described in other mouse models of aortic aneurysm and dissection. ${ }^{32}$ Unlike other mouse models with reported human TAAD mutations, no spontaneous aneurysm was observed in the Mmp17-deficient mice, a finding likely related to the mild hypotension in these animals. However, increased vessel wall stress induced by infusion of angiotensin II increased the susceptibility of Mmp17-null mice to thoracic (but not abdominal) aortic aneurysm. In several mouse models of aortic aneurysm, although VSMC are dedifferentiated or have synthetic-like phenotype, no significant effects are found on the protein or mRNA expression of true VSMC differentiation markers such as SM-MHC or calponin. ${ }^{32}$ Likewise, we found no significant effect on the expression levels of these markers in Mmp17null VSMCs, in spite of their striking alterations revealed by TEM. Our data demonstrate that VSMCs in the mouse aorta can express normal levels of contractile markers and yet be dysfunctional due to their misorientation within the arterial vessel wall, thus impacting VSMC-ECM mechanical coupling. Mechano-sensing defects in the aortic wall have recently been proposed as a common mechanism to explain the variety of genetic mutations leading to similar pathological outcomes in aortic pathology, ${ }^{3}$ and findings in Mmp17-null arteries in fact indicate a defective elastic recoil/compliance. Impaired VSMC differentiation in $M m p 17^{-/}$mice could also be related to the altered ECM organization, which might directly decrease Tgfb 1 availability. ${ }^{33}$ Proteome analysis did not reveal changes in the levels of the contractile proteins SM-MHC or calponin, but did indicate an important contribution to the phenotype from alterations to actomyosin and cell-adhesion pathways likely related to matrix/force-dependent responses. Proteomics analysis also identified mitochondrial dysfunction in the de-differentiated phenotype of Mmp17-null aortic VSMCs, both in neonates and adults. Given the links between mitochondrial function and TGFB signaling in arterial tortuosity syndrome, ${ }^{34}$ the putative contribution of impaired mitochondrial fitness to aortic aneurysms deserves further investigation.

MMPs are often found expressed at high levels in aneurysms, and are proposed to contribute to disease progression by direct cleavage of ECM components or by affecting VSMC phenotype. ${ }^{12}$ A wellestablished example is Mmp12 (elastase), which is directly related to aneurysm formation in mouse models. ${ }^{15}$ Also, the membrane-type MMP Mmp14 (Mt1-mmp) is upregulated during pathological vascular remodeling and contributes to PDFGB/PDGFR $\beta$-dependent VSMC de-differentiation. ${ }^{13,14,35}$ MMP17 overexpression in tumor cells affects the covering of vascular endothelial cells by pericytes, perivascular cells related to VSMC, ${ }^{36}$ and the zebrafish homolog Mmp17b is required for migration of neural crest cells, which contribute to VSMCs in certain aortic regions. ${ }^{37}$ Despite these findings and the reported expression of several MMPs during mouse aorta development, ${ }^{16}$ no other MMP mutations or MMP-deficient mouse models have been shown to produce aortic aneurysm. ${ }^{1,38}$ Moreover, we observed no major changes in other MMP family members (Mmp2, Mmp3, Mmp7, Mmp14, Mmp19, and Mmp25) or in tissue inhibitors of MMPs (TIMPs) in Mmp17-null aortas at E14.5, $\mathrm{P} 0$, or P7, indicating that the lack of Mmp17 activity is not compensated by related proteases (unpublished data). 
Our lentivirus rescue experiments showed that Mmp17 function in the arterial wall required its catalytic activity. This action occurred mainly during embryonic development and involved the spatiotemporal orchestration of signals through the cleavage of Opn, a matricellular protein that can bind cell receptors such as integrins and matrix proteins. ${ }^{27}$ The role of OPN in the vessel wall has mainly been recognized in the context of vascular pathologies such as aneurysm, atherosclerosis and vascular calcification. ${ }^{27}$ Opn-null and Opnoverexpressing transgenic mice have vascular phenotypes that resemble those of Mmp17-null aortas: pressureinduced increased lumen diameter and low blood pressure in Opn-null mice and media thickening and high numbers of VSMCs without injury in Opn-transgenic mice. ${ }^{28,39}$ Despite these observations and the reported expression of Opn in embryonic rat aorta, ${ }^{30}$ its function in vessel wall development has remained unclear. We detected Opn mainly in the peripheral layer of the embryonic mouse aorta from E14.5 to E18.5, where scattered Mmp17 positive cells are also found. N-terminal and C-terminal Opn fragments modulate migration and proliferation via distinct signaling pathways and in fact we observed below-normal JNK activation in Mmp17null embryonic VSMCs. ${ }^{27,40}$ The rescue in neonates, by lentiviral expression of the N-terminal Opn fragment, of JNK signaling and normal VSMC proliferation, demonstrated that spatiotemporally defined cleavage of Opn by Mmp17 is required for proper VSMC migration and distribution during aorta development. The cellular events required for the formation of a multilayered arterial vessel wall are proposed to be regulated by a gradient of soluble signals including PDFGB. ${ }^{5}$ Since PDGFB-induced migration of arterial VSMCs requires Opn secretion, ${ }^{41}$ our data are consistent with a regulatory circuit in which PDGFB stimulates Opn production in embryonic VSMCs, followed by Mmp17-dependent Opn cleavage to ensure proper VSMC migration and reorientation. Binding of $\alpha v \beta 3$ integrin to the Opn N-terminal RGD site will lead to mechanical stretch stimulation of $\mathrm{JNK}_{;}^{.42}$ in this regard, inhibition of phospho-JNK has been shown to decrease Opn-induced VSMC migration. ${ }^{43}$

Genetic understanding of TAAD etiology is shifting therapies toward gene-tailored management by interfering either with the angiotensin/TGFB axis or the non-canonical TGFB signaling with ERK (extracellular signal-regulated kinases) or mTOR (mammalian target of rapamycin) inhibitors. ${ }^{1,32}$ In addition, gene therapy for human disease was recently approved, and gene therapy-based interventions are being considered as a viable alternative for treatment of certain cardiovascular diseases. ${ }^{44,45}$ The present study shows the therapeutic potential of reexpressing Mmp17 or the Opn N-terminal fragment in Mmp17-null VSMCs both as an early intervention and also in established lesions in mice and the possible application in TAAD patients with mutations in MMP17. The use of a loss-of-function mouse model and lentiviral-rescue approaches provide evidence of direct contributions by Mmp17 and Opn to arterial vessel wall development and function, and it is possible that Mmp17-dependent processing of undetectable amounts of Opn, or other potential substrates such as Thbs4 (unpublished data), would be sufficient to partially recover the structure and function of the adult vasculature, resulting in reestablishment of vessel wall properties after lentiviral injection. In addition, we propose that diagnosis of MMP17 mutations could help to identify patients who might eventually benefit from such tailored-gene therapy approaches. 


\section{ACKNOWLEDGEMENTS}

We thank the SIdI-UAM Electron Microscopy Unit, the CNIC Advanced Imaging Unit for MRI studies, Cristina Clemente and Francesc Canals for SILAC analysis, Pilar Gonzalo for technical support and manuscript editing, Jesús Borreguero and Lorena Flores Ruiz for ultrasound analysis, Rubén A. Mota, Ángel Colmenar, Laura Balonga, Raquel Sánchez Díaz and Silvia Lorrio for technical support, the Brant Isakson laboratory and Robert P. Mecham for helpful discussions, Per Fogelstrand for the VSMC isolation protocol, Juan M. Zapata for providing bortezomib, and Simon Bartlett for English editing.

\section{SOURCES OF FUNDING}

This work was supported by grants from the Ministerio de Economía y Competitividad (RD12/0042/0022 to JMR, RD12/0042/0024 to MS, and RD12/0042/0023 to AGA [FEDER cofounded], and SAF2011-25619 to AGA). MM-A was funded by a MINECO fellowship. SILAC analysis was performed in the Proteomics Laboratory at Vall d'Hebron Institute of Oncology (VHIO), a member of ProteoRed, PRB2-ISCIII, supported by grant PT13/0001. The CNIC is supported by the Ministerio de Economía y Competitividad and the ProCNIC Foundation.

\section{DISCLOSURES}

None.

\section{AUTHOR CONTRIBUTIONS}

M.M-A. designed experiments, performed research and wrote the paper; A.B.G-R. and M.S. performed myography studies; D.G. and D.M. performed human genetic analysis; N.M-B. provided technical support in angiotensin II experiments; A.P. provided technical support; C.S-C. performed embryo LacZ staining; F.M. performed in silico modeling; E.C. and J.V. performed proteomics analysis; A.A. and J.M.R. helped with the lentiviral approach; and A.G.A. designed the research and wrote the paper. 


\section{REFERENCES}

1. Gillis E, Van Laer L, Loeys BL. Genetics of thoracic aortic aneurysm: At the crossroad of transforming growth factor-beta signaling and vascular smooth muscle cell contractility. Circ Res. 2013;113:327-340

2. Milewicz DM, Guo DC, Tran-Fadulu V, Lafont AL, Papke CL, Inamoto S, Kwartler CS, Pannu H. Genetic basis of thoracic aortic aneurysms and dissections: Focus on smooth muscle cell contractile dysfunction. Annu Rev Genomics Hum Genet. 2008;9:283-302

3. Humphrey JD, Milewicz DM, Tellides G, Schwartz MA. Cell biology. Dysfunctional mechanosensing in aneurysms. Science. 2014;344:477-479

4. Majesky MW. Developmental basis of vascular smooth muscle diversity. Arterioscler Thromb Vasc Biol. 2007;27:1248-1258

5. Greif DM, Kumar M, Lighthouse JK, Hum J, An A, Ding L, Red-Horse K, Espinoza FH, Olson L, Offermanns S, Krasnow MA. Radial construction of an arterial wall. Dev Cell. 2012;23:482-493

6. Dietz HC, Cutting GR, Pyeritz RE, Maslen CL, Sakai LY, Corson GM, Puffenberger EG, Hamosh A, Nanthakumar EJ, Curristin SM, et al. Marfan syndrome caused by a recurrent de novo missense mutation in the fibrillin gene. Nature. 1991;352:337-339

7. Loeys BL, Schwarze U, Holm T, Callewaert BL, Thomas GH, Pannu H, De Backer JF, Oswald GL, Symoens S, Manouvrier S, Roberts AE, Faravelli F, Greco MA, Pyeritz RE, Milewicz DM, Coucke PJ, Cameron DE, Braverman AC, Byers PH, De Paepe AM, Dietz HC. Aneurysm syndromes caused by mutations in the tgf-beta receptor. N Engl J Med. 2006;355:788-798

8. Inamoto S, Kwartler CS, Lafont AL, Liang YY, Fadulu VT, Duraisamy S, Willing M, Estrera A, Safi H, Hannibal MC, Carey J, Wiktorowicz J, Tan FK, Feng XH, Pannu H, Milewicz DM. Tgfbr2 mutations alter smooth muscle cell phenotype and predispose to thoracic aortic aneurysms and dissections. Cardiovasc Res. 2010;88:520-529

9. Milewicz DM, Regalado ES, Shendure J, Nickerson DA, Guo DC. Successes and challenges of using whole exome sequencing to identify novel genes underlying an inherited predisposition for thoracic aortic aneurysms and acute aortic dissections. Trends Cardiovasc Med. 2014;24:53-60

10. Regalado ES, Guo DC, Villamizar C, Avidan N, Gilchrist D, McGillivray B, Clarke L, Bernier F, Santos-Cortez RL, Leal SM, Bertoli-Avella AM, Shendure J, Rieder MJ, Nickerson DA, Project NGES, Milewicz DM. Exome sequencing identifies smad3 mutations as a cause of familial thoracic aortic aneurysm and dissection with intracranial and other arterial aneurysms. Circ Res. 2011;109:680-686

11. Page-McCaw A, Ewald AJ, Werb Z. Matrix metalloproteinases and the regulation of tissue remodelling. Nat Rev Mol Cell Biol. 2007;8:221-233

12. Theruvath TP, Jones JA, Ikonomidis JS. Matrix metalloproteinases and descending aortic aneurysms: Parity, disparity, and switch. J Card Surg. 2012;27:81-90

13. Lehti K, Allen E, Birkedal-Hansen H, Holmbeck K, Miyake Y, Chun TH, Weiss SJ. An mt1-mmppdgf receptor-beta axis regulates mural cell investment of the microvasculature. Genes Dev. 2005;19:979-991

14. Lehti K, Rose NF, Valavaara S, Weiss SJ, Keski-Oja J. Mt1-mmp promotes vascular smooth muscle dedifferentiation through lrp1 processing. J Cell Sci. 2009;122:126-135

15. Longo GM, Buda SJ, Fiotta N, Xiong W, Griener T, Shapiro S, Baxter BT. Mmp-12 has a role in abdominal aortic aneurysms in mice. Surgery. 2005;137:457-462

16. McLean SE, Mecham BH, Kelleher CM, Mariani TJ, Mecham RP. Extracellular matrix gene expression in the developing mouse aorta. In: Miner JH, ed. Extracellular Matrix in Development and Disease. San Diego, CA: Elsevier; 2005. Advances in Developmental Biology; vol. 15:81-128

17. Rikimaru A, Komori K, Sakamoto T, Ichise H, Yoshida N, Yana I, Seiki M. Establishment of an mt4-mmp-deficient mouse strain representing an efficient tracking system for $\mathrm{mt} 4-\mathrm{mmp} / \mathrm{mmp}-17$ expression in vivo using beta-galactosidase. Genes Cells. 2007;12:1091-1100 
18. Sohail A, Sun Q, Zhao H, Bernardo MM, Cho JA, Fridman R. Mt4-(mmp17) and mt6-mmp (mmp25), a unique set of membrane-anchored matrix metalloproteinases: Properties and expression in cancer. Cancer Metastasis Rev. 2008;27:289-302

19. Jackson V, Olsson T, Kurtovic S, Folkersen L, Paloschi V, Wagsater D, Franco-Cereceda A, Eriksson P. Matrix metalloproteinase 14 and 19 expression is associated with thoracic aortic aneurysms. J Thorac Cardiovasc Surg. 2012;144:459-466

20. Carrell TW, Burnand KG, Wells GM, Clements JM, Smith A. Stromelysin-1 (matrix metalloproteinase-3) and tissue inhibitor of metalloproteinase-3 are overexpressed in the wall of abdominal aortic aneurysms. Circulation. 2002;105:477-482

21. Lenk GM, Tromp G, Weinsheimer S, Gatalica Z, Berguer R, Kuivaniemi H. Whole genome expression profiling reveals a significant role for immune function in human abdominal aortic aneurysms. BMC Genomics. 2007;8:237

22. Sohail A, Marco M, Zhao H, Shi Q, Merriman S, Mobashery S, Fridman R. Characterization of the dimerization interface of membrane type 4 (mt4)-matrix metalloproteinase. J Biol Chem. 2011;286:33178-33189

23. Scholz D, Ito W, Fleming I, Deindl E, Sauer A, Wiesnet M, Busse R, Schaper J, Schaper W. Ultrastructure and molecular histology of rabbit hind-limb collateral artery growth (arteriogenesis). Virchows Arch. 2000;436:257-270

24. O'Connell MK, Murthy S, Phan S, Xu C, Buchanan J, Spilker R, Dalman RL, Zarins CK, Denk W, Taylor CA. The three-dimensional micro- and nanostructure of the aortic medial lamellar unit measured using 3d confocal and electron microscopy imaging. Matrix Biol. 2008;27:171-181

25. Owens GK, Kumar MS, Wamhoff BR. Molecular regulation of vascular smooth muscle cell differentiation in development and disease. Physiol Rev. 2004;84:767-801

26. Esteban V, Mendez-Barbero N, Jimenez-Borreguero LJ, Roque M, Novensa L, Garcia-Redondo AB, Salaices M, Vila L, Arbones ML, Campanero MR, Redondo JM. Regulator of calcineurin 1 mediates pathological vascular wall remodeling. J Exp Med. 2011;208:2125-2139

27. Scatena M, Liaw L, Giachelli CM. Osteopontin: A multifunctional molecule regulating chronic inflammation and vascular disease. Arterioscler Thromb Vasc Biol. 2007;27:2302-2309

28. Isoda K, Nishikawa K, Kamezawa Y, Yoshida M, Kusuhara M, Moroi M, Tada N, Ohsuzu F. Osteopontin plays an important role in the development of medial thickening and neointimal formation. Circ Res. 2002;91:77-82

29. Gao H, Steffen MC, Ramos KS. Osteopontin regulates alpha-smooth muscle actin and calponin in vascular smooth muscle cells. Cell Biol Int. 2012;36:155-161

30. Giachelli C, Bae N, Lombardi D, Majesky M, Schwartz S. Molecular cloning and characterization of $2 \mathrm{~b} 7$, a rat mrna which distinguishes smooth muscle cell phenotypes in vitro and is identical to osteopontin (secreted phosphoprotein i, 2ar). Biochem Biophys Res Commun. 1991;177:867-873

31. Bilato C, Curto KA, Monticone RE, Pauly RR, White AJ, Crow MT. The inhibition of vascular smooth muscle cell migration by peptide and antibody antagonists of the alphavbeta3 integrin complex is reversed by activated calcium/calmodulin- dependent protein kinase ii. $J$ Clin Invest. 1997; 100:693-704

32. Li W, Li Q, Jiao Y, Qin L, Ali R, Zhou J, Ferruzzi J, Kim RW, Geirsson A, Dietz HC, Offermanns $\mathrm{S}$, Humphrey JD, Tellides G. Tgfbr2 disruption in postnatal smooth muscle impairs aortic wall homeostasis. J Clin Invest. 2014; 124:755-767

33. Klingberg F, Chow ML, Koehler A, Boo S, Buscemi L, Quinn TM, Costell M, Alman BA, Genot E, Hinz B. Prestress in the extracellular matrix sensitizes latent tgf-beta1 for activation. J Cell Biol. 2014;207:283-297

34. Willaert A, Khatri S, Callewaert BL, Coucke PJ, Crosby SD, Lee JG, Davis EC, Shiva S, Tsang M, De Paepe A, Urban Z. Glut10 is required for the development of the cardiovascular system and the notochord and connects mitochondrial function to tgfbeta signaling. Hum Mol Genet. 2012;21:1248-1259 
35. Filippov S, Koenig GC, Chun TH, Hotary KB, Ota I, Bugge TH, Roberts JD, Fay WP, BirkedalHansen H, Holmbeck K, Sabeh F, Allen ED, Weiss SJ. Mt1-matrix metalloproteinase directs arterial wall invasion and neointima formation by vascular smooth muscle cells. J Exp Med. 2005;202:663-671

36. Chabottaux V, Sounni NE, Pennington CJ, English WR, van den Brule F, Blacher S, Gilles C, Munaut C, Maquoi E, Lopez-Otin C, Murphy G, Edwards DR, Foidart JM, Noel A. Membranetype 4 matrix metalloproteinase promotes breast cancer growth and metastases. Cancer Res. 2006;66:5165-5172

37. Leigh NR, Schupp MO, Li K, Padmanabhan V, Gastonguay A, Wang L, Chun CZ, Wilkinson GA, Ramchandran R. Mmp17b is essential for proper neural crest cell migration in vivo. PLoS One. 2013;8:e76484

38. Hoffjan S. Genetic dissection of marfan syndrome and related connective tissue disorders: An update 2012. Mol Syndromol. 2012;3:47-58

39. Myers DL, Harmon KJ, Lindner V, Liaw L. Alterations of arterial physiology in osteopontin-null mice. Arterioscler Thromb Vasc Biol. 2003;23:1021-1028

40. Christensen B, Klaning E, Nielsen MS, Andersen MH, Sorensen ES. C-terminal modification of osteopontin inhibits interaction with the alphavbeta3-integrin. J Biol Chem. 2012;287:3788-3797

41. Jalvy S, Renault MA, Leen LL, Belloc I, Bonnet J, Gadeau AP, Desgranges C. Autocrine expression of osteopontin contributes to pdgf-mediated arterial smooth muscle cell migration. Cardiovasc Res. 2007; 75:738-747

42. Katsumi A, Naoe T, Matsushita T, Kaibuchi K, Schwartz MA. Integrin activation and matrix binding mediate cellular responses to mechanical stretch. J Biol Chem. 2005;280:16546-16549

43. Yu HW, Liu QF, Liu GN. Positive regulation of the egr-1/osteopontin positive feedback loop in rat vascular smooth muscle cells by tgf-beta, erk, jnk, and p38 mapk signaling. Biochem Biophys Res Commun. 2010;396:451-456

44. Salmon F, Grosios K, Petry H. Safety profile of recombinant adeno-associated viral vectors: Focus on alipogene tiparvovec (glybera(r)). Expert Rev Clin Pharmacol. 2014; 7:53-65

45. Zsebo K, Yaroshinsky A, Rudy JJ, Wagner K, Greenberg B, Jessup M, Hajjar RJ. Long-term effects of aav1/serca2a gene transfer in patients with severe heart failure: Analysis of recurrent cardiovascular events and mortality. Circ Res. 2014;114:101-108 


\section{FIGURE LEGENDS}

Figure 1. R373H mutation in MMP17 in human TAAD impairs protein expression. A, Protein domain structure of human MMP17. The arrow marks the mutation found in a TAAD patient at Arg373. B, 3D tertiary protein structures of the lowest energy models for human wild-type MMP17 (left panels) and the R373H mutant (right). Monomers (upper panels) and homodimers (lower panels) are shown. The same colors used for the domain structure (A) are used in the 3D models (orange, catalytic center; red, C-terminal residue). Domains with a different orientation in the wild-type and mutant versions are shown in blue. C, Quantitative-reverse transcriptase-PCR (Q-RT-PCR) analysis of MMP17 mRNA levels in HEK293 cells non-transfected (control) or transfected with wild-type or R373H mutated human MMP17, presented as calibrated normalized relative quantity (CNRQ) obtained in qBase analysis; $\mathrm{n}=4$ independent experiments, mean \pm s.e.m. $\mathbf{D}$, Representative western-blot of MMP17 protein expression in HEK293 cells transfected with wild-type or R373H mutated human MMP17. E, Representative confocal microscope images of transfected HEK293 cells with or without treatment with bortezomib. Cells were stained with anti-MMP17 antibody (green) and Hoechst (blue). Scale bar $20 \mu \mathrm{m} . \mathrm{n}=5$ independent experiments.

Figure 2. $\mathrm{Mmp17}^{-/-}$mice are more susceptible to thoracic aortic aneurysm in response to angiotensin II. A, Representative magnetic resonance images of thoracic descending aorta (left) and lumen diameter quantification (right) in wild-type or $M m p 17^{-/}$adult mice (12 weeks old; $\mathrm{n}=8$ mice per genotype). A, aorta lumen; scale bar $1 \mathrm{~mm}$. B, Systolic and diastolic blood pressure and heart rate in 8-week-old wild-type and Mmp1 $17^{-/-}$mice (representative data are shown from one of 4 independent experiments with similar results; $\mathrm{n}=$ 10 mice per genotype). C, Ultrasound-measured lumen diameter of thoracic ascending aorta (aortic arch) $(n=14$ mice per genotype tested in two independent experiments). D, Representative pictures of thoracic aortas from

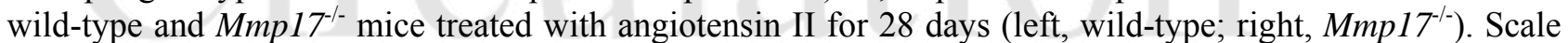
bar $2000 \mu \mathrm{m}$. E, Histological transverse cuts of ascending thoracic aortas (aortic arch) from angiotensin IItreated wild-type (left) and $M m p 17^{-/-}$mice (right). Scale bar $500 \mu \mathrm{m}$. F, Ultrasound-measured lumen diameter of abdominal aorta ( $\mathrm{n}=14$ mice per genotype tested in two independent experiments). G, Representative pictures of abdominal aortas from wild-type and $M m p 17^{-/-}$mice treated with angiotensin II for 28 days (left, wild-type; right, $\mathrm{Mmp} \mathrm{7}^{-/}$). Scale bar $2000 \mu \mathrm{m}$. H, Histological transverse cuts of abdominal aortas from angiotensin II-treated wild-type (left) and $\mathrm{Mmp} \mathrm{7}^{-/ \mathrm{r}}$ mice (right). Scale bar $500 \mu \mathrm{m}$. Numerical data are means \pm s.e.m. Data in $\mathbf{A}$ and $\mathbf{B}$ were tested by Student's $t$-test $\left(* \mathrm{p}<0.05,{ }^{*} \mathrm{p}<0.01, * * * \mathrm{p}<0.001\right)$ and data in $\mathbf{C}$ and $\mathbf{F}$ were compared by two-way ANOVA followed by Bonferroni post-test.

Figure 3. Arteries from $M m p 17^{-/-}$mice contain aberrant and mis-oriented VSMCs. A, Representative TEM images of ultrathin transverse (top) and longitudinal (bottom) sections of thoracic aorta. Note the differences in VSMC morphology, cell-ECM connections (arrows), and cell-cell connections (arrowheads) between wild-type and $M m p 17^{-/}$aortas. $M m p 17^{-/}$mice also showed excess ECM (asterisks) and frayed elastin layers (inset, right panel). Scale bar $4 \mu \mathrm{m} . \mathrm{n}=10$ mice per genotype. IEL, internal elastic lamina; N, nuclei. B, Representative confocal microscopy images of SM-MHC and calponin staining (white), elastin autofluorescence (green) and nuclear Hoechst staining (blue) in transverse aortic sections from wild-type or Mmp $17^{-/}$mice. Scale bar $20 \mu \mathrm{m} . \mathrm{n}=7$ mice per genotype. C, Density of cell nuclei in transverse sections of wild-type and $M m p 17^{-/}$aorta stained with Hoechst and elastin auto-fluorescence (nuclei from endothelial and adventitial cells were excluded) ( $\mathrm{n}=30$ mice per genotype analyzed in 5 independent experiments). D, Numbers of VSMCs freshly isolated from wild-type or $M m p 17^{-/}$aortas ( $\mathrm{n}=8$ aortas per experiment and genotype in 7 independent experiments). E, Representative images of transverse sections of thoracic aorta from wild-type and $M m p 17^{-/-}$mice stained with hematoxylin and eosin (left) (scale bar $50 \mu \mathrm{m}$ ) fixed under pressure (top) or fixed after perfusion (bottom) and media layer thickness (right) and its quantification; $\mathrm{n}=15$ mice per genotype analyzed in 3 independent experiments. Numerical data are means \pm s.e.m., and were tested by unpaired Student's $t$-test $(* \mathrm{p}<0.05, * * \mathrm{p}<0.01)$. 
Figure 4. Perinatal onset of the arterial phenotype of $\mathbf{M m p 1 7}^{-/-}$mice. A, Representative TEM images of ultrathin transverse (top) and longitudinal (bottom) aortic sections from neonate (P0) wild-type and Mmp17 ${ }^{-/-}$ mice. Note the cell-ECM connections (arrows) in wild-type tissue, contrasting with the abundance of microfibril bundles (arrows) and cellular debris (asterisks) in $M m p 17^{-/-}$aortas. Scale bars, 5 and $0.5 \mu \mathrm{m}$ for transverse and 5 and $2 \mu \mathrm{m}$ for longitudinal sections ( $\mathrm{n}=3$ mice per genotype). $\mathbf{B}$, Representative confocal microscope images of aortas from 7-day-old (P7) wild-type and $\mathrm{Mmp}^{-/ /}$mice, showing anti-pH3 immunostaining (red), elastin auto-fluorescence (green) and Hoechst nuclear staining (blue). Scale bar $20 \mu \mathrm{m}$. White arrowheads highlight $\mathrm{pH} 3$ positive nuclei. $\mathbf{C}$, Numbers of $\mathrm{pH} 3$-positive nuclei in the media layer of aortas from P0 to P30; $\mathrm{n}=15-17$ mice per genotype and time point analyzed in 3 independent experiments. D, Q-RT-PCR analysis of Tgfb2 mRNA levels in P7 total aorta extracts, presented as fold change from calibrated normalized relative quantity (CNRQ) obtained in qBase analysis; $\mathrm{n}=21$ mice per genotype analyzed in 3 independent experiments. Numerical data are means \pm s.e.m., and were tested by one-way ANOVA in $\mathbf{C}(* * *$ $\mathrm{p}<0.001)$ and one-sample $t$-test in $\mathbf{D}(* * \mathrm{p}<0.01)$.

Figure 5. Quantitative proteomics comparison of the proteomes of aortas from wild-type and $M m p 17^{-/-}$ neonatal (A) and adult (B) mice. Proteins were extracted from 7 pooled aortas per genotype at P7 and from 3 pooled aortas per genotype in adults. Protein pools were digested, and the resulting peptides were subjected to relative quantification by mass spectrometry in multiplexed mode using isobaric tag for relative and absolute quantification (iTRAQ) labeling. The quantitative protein data were grouped into gene ontology (GO) functional categories and the data were analyzed to determine significant category changes. Cumulative frequency distributions of standardized $\log 2$ ratios of proteins $(\mathrm{Zq})$ were plotted to show the coordinated alterations (up or down) in proteins belonging to the changing categories. These functional categories showed an abundance decrease $(\mathrm{Zq}>0$, right shift of sigmoidal curves, blue) or an abundance increase $(\mathrm{Zq}<0$, left shift of curves, red) in Mmp17-null mice relative to the wild-type mice. The dashed lines represent the nullhypothesis distribution, and the black lines show the distribution of all proteins quantified in each experiment.

Figure 6. Expression of catalytically active Mmp17 in $M m p 17^{-/-}$mice prevents aortic phenotype. A, Design of the lentiviral (LV) vector. The vector directs SFFV (spleen focus-forming virus)-driven Mmp17 expression and IRES (internal ribosome entry site)-driven expression of GFP. B, Lentiviral rescue strategy. Lentivirus was injected one day after birth (P1) and mice were sacrificed and analyzed for different parameters 7 days or 8 weeks later. C, Numbers of pH3-positive VSMCs 7 days after lentiviral injection of mock (empty vector encoding GFP), wild-type Mmp17 or E248A mutant Mmp17 into wild-type or Mmp17 ${ }^{-/ 2}$ mice $(\mathrm{n}=10$ 19 mice per condition analyzed in 3 independent experiments). D-F, Diastolic blood pressure, density of cell nuclei, and media thickness in non-pressure fixed aortas 8 weeks after injection with mock, wild-type Mmp17 or E248A mutant Mmp17 lentivirus ( $\mathrm{n}=12-20$ mice per condition analyzed in 4 independent experiments). G, Representative TEM images of thoracic aortic sections 8 weeks after injection with mock, wild-type Mmp17 or E248A mutant Mmp17 lentivirus ( $n=4$ mice per condition analyzed in 3 independent experiments). Scale bar $5 \mu \mathrm{m}$. H, Representative confocal microscopy images of calponin immunostaining (white), elastin auto-fluorescence (green), and Hoechst nuclear staining (blue) in thoracic aortic sections 8 weeks after injection with mock, wild-type Mmp17 or E248A mutant Mmp17 lentivirus ( $\mathrm{n}=10$ mice per condition analyzed in 3 independent experiments). Scale bar $20 \mu \mathrm{m}$. Data in $\mathbf{C - F}$ are presented as means \pm s.e.m., and were tested by one-way ANOVA followed by Tukey's multiple comparison test $\left(^{*} \mathrm{p}<0.05,{ }^{*} \mathrm{p}<0.01,{ }^{* * *}\right.$ $\mathrm{p}<0.001)$.

Figure 7. Osteopontin (Opn) cleavage by Mmp17 is required for JNK activation in VSMC during aorta development. A, Representative western-blot of human OPN after in vitro digestion by human recombinant MMP17. Full length OPN and its C-terminal fragment are detected with the OPN antibody anti-C-terminal (1H3F7). $\mathrm{n}=7$ independent experiments. B, Scheme of human OPN protein. The cleavage site for human MMP17 is localized between 210 and 211 residues (arrow). C, In silico model of OPN (purple) bound to human MMP17 homodimer (green). Note the location of the OPN cleavage site (yellow) close to the MMP17 catalytic center (orange). D, Representative confocal microscopy images of E14.5 Mmp17-null aortas stained 
for Opn (red), with nuclear Hoechst staining in blue. Scale bar $20 \mu \mathrm{m}$. (n = 5-7 embryos per genotype). E, Representative western-blot of Opn in aortic extracts from E14.5 wild-type (+/+) and Mmp1 $7^{-/-}(-/-)$embryos (pool of 8-10 embryonic aortas or sternal cartilage pieces per genotype). The chart shows average fold-change of the quantification of the bands corresponding to full length (FL) and the N-terminal Opn fragment (N-term) as indicated in the western-blot; $\mathrm{n}=3$ independent experiments. F, Graph shows quantification (fold change) of Opn mRNA levels by Q-RT-PCR in aortic extracts from E14.5 wild-type (+/+) and Mmp17/- (-/-) embryos (pool of 4-10 embryonic aortas; $n=4$ independent experiments). $\mathbf{G}$, Representative confocal microscopy images of E14.5 wild-type and Mmp17-null aortas stained for phospho-JNK (red) and CD31 (white), with nuclear Hoechst staining in blue ( $\mathrm{n}=5-7$ embryos per genotype). Scale bar $50 \mu \mathrm{m}$ (left) and $20 \mu \mathrm{m}$ (right). Numerical data in $\mathbf{E}$ and $\mathbf{F}$ are means \pm s.e.m. and were tested by one-sample t-test.

Figure 8. Lentiviral injection of $\mathrm{N}$-terminal Opn fragment rescues VSMC proliferation and JNK signaling in $\mathbf{M m p 1 7}^{-/-}$neonatal aortas. A, LV design showing full length and N-terminal mouse Opn constructs. B, Representative confocal images of $\mathrm{pH} 3$ staining (red) in P8 aortas from Mmp17-null mice injected at P1 with N-terminal (N-term), full length (FL) Opn or control LV (left) and quantification of $\mathrm{pH} 3$ positive cells (right). ( $\mathrm{n}=16$ to 20 mice per condition, analyzed in two independent experiments). D, Representative confocal images of P8 aortas from null mice injected with LV as in $\mathbf{B}$, stained for phosphoJNK (green) (left) and quantification of average fluorescence intensity in the arterial media (right); nuclear Hoechst staining in blue. ( $\mathrm{n}=16$ to 20 mice were analyzed per condition in two independent experiments). Numerical data are means \pm s.e.m. and were tested by one-way ANOVA followed by Tukey's multiple comparison test $(* * \mathrm{p}<0.01, * * * \mathrm{p}<0.001)$.

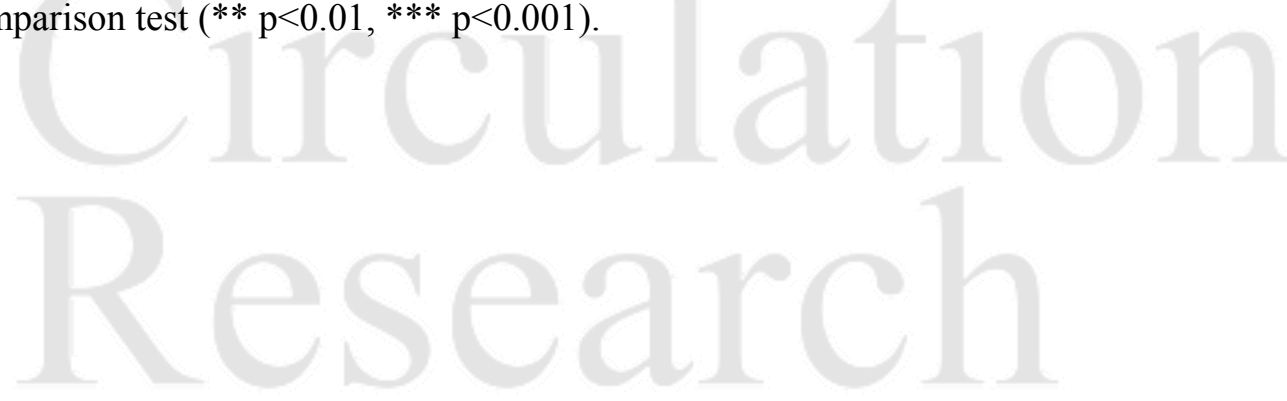




\section{Novelty and Significance}

\section{What Is Known?}

- Aortic aneurysms result from alterations to extracellular matrix (ECM) assembly and/or vascular smooth muscle cell (VSMC) maturation in the aortic vessel wall.

- Matrix metalloproteinases (MMP) are considered active players in the progression of pathological vascular remodeling.

- Osteopontin (OPN) is a matricellular protein with recognized functions in vascular pathology.

\section{What New Information Does This Article Contribute?}

- The absence of the matrix metalloproteinase Mmp17 affects the vascular smooth muscle cell phenotype in the aorta and results in increased predisposition to thoracic aortic aneurysms.

- A novel molecular axis involving Mmp17/Opn cleavage and phospho-JNK signaling is shown to be required for normal VSMC maturation and distribution during aorta development.

- Lentiviral-based expression of active Mmp17 or the N-terminal OPN fragment rescues the aortic VSMC phenotype in Mmp17-null mice.

Aortic aneurysms are associated with high mortality due to increased risk of aorta dissection and rupture. These disorders are often associated with mutations in genes that impact VSMC differentiation and contractility or ECM composition and assembly, thus affecting the mechanics of the vessel wall. Novel therapies are mostly focused on the inhibition of components of the angiotensin II/ TGFbeta signaling pathway. In this study we identify the protease Mmp17 as required for proper VSMC maturation and distribution in the aortic wall. Mutations found in the MMP17 gene in thoracic aortic aneurysm patients suggested that the absence of MMP17 increases predisposition to TAA, and this was confirmed in a genetic loss-of-function model in mice. We have also delineated a novel molecular pathway in which Mmp17dependent cleavage of the matricellular protein OPN is required for activation of phospho-JNK in VSMC, regulating their normal maturation and distribution in the aorta during embryonic development. Lentiviralbased expression of active Mmp17 or the N-terminal fragment of Opn rescues the VSMC phenotype in the aortas of MMP17-deficient mice, opening potential options for personalized gene therapy in these lifethreatening disorders. 
A

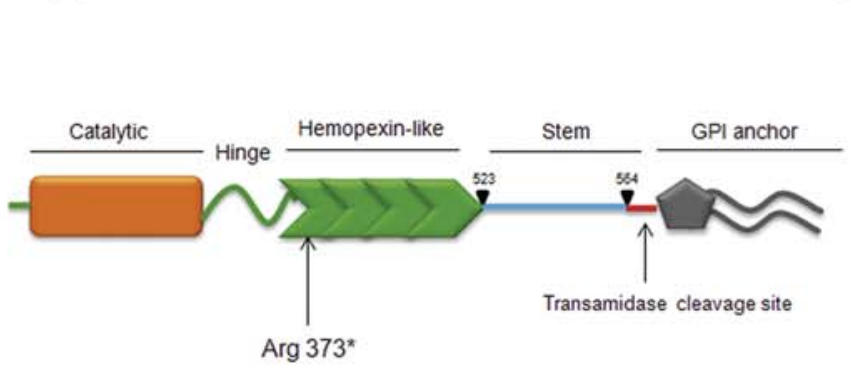

C

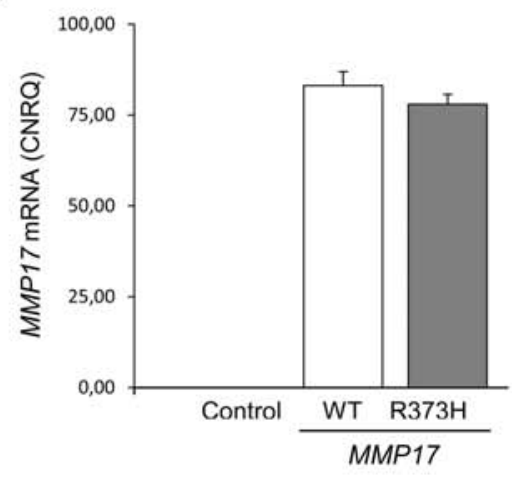

B

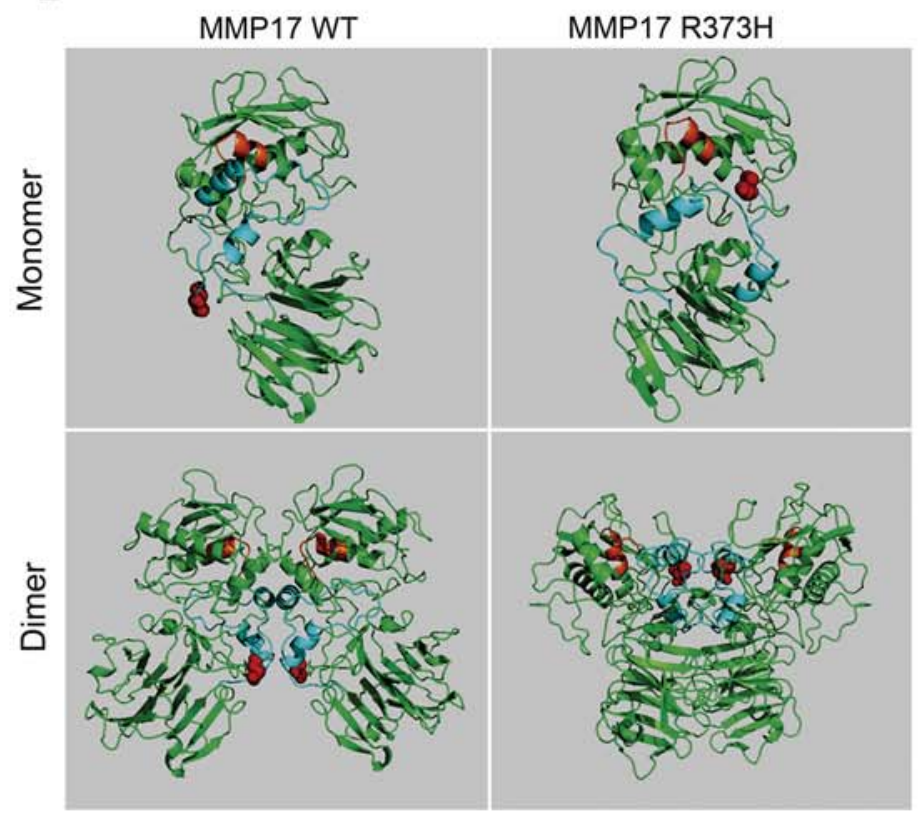

E

D
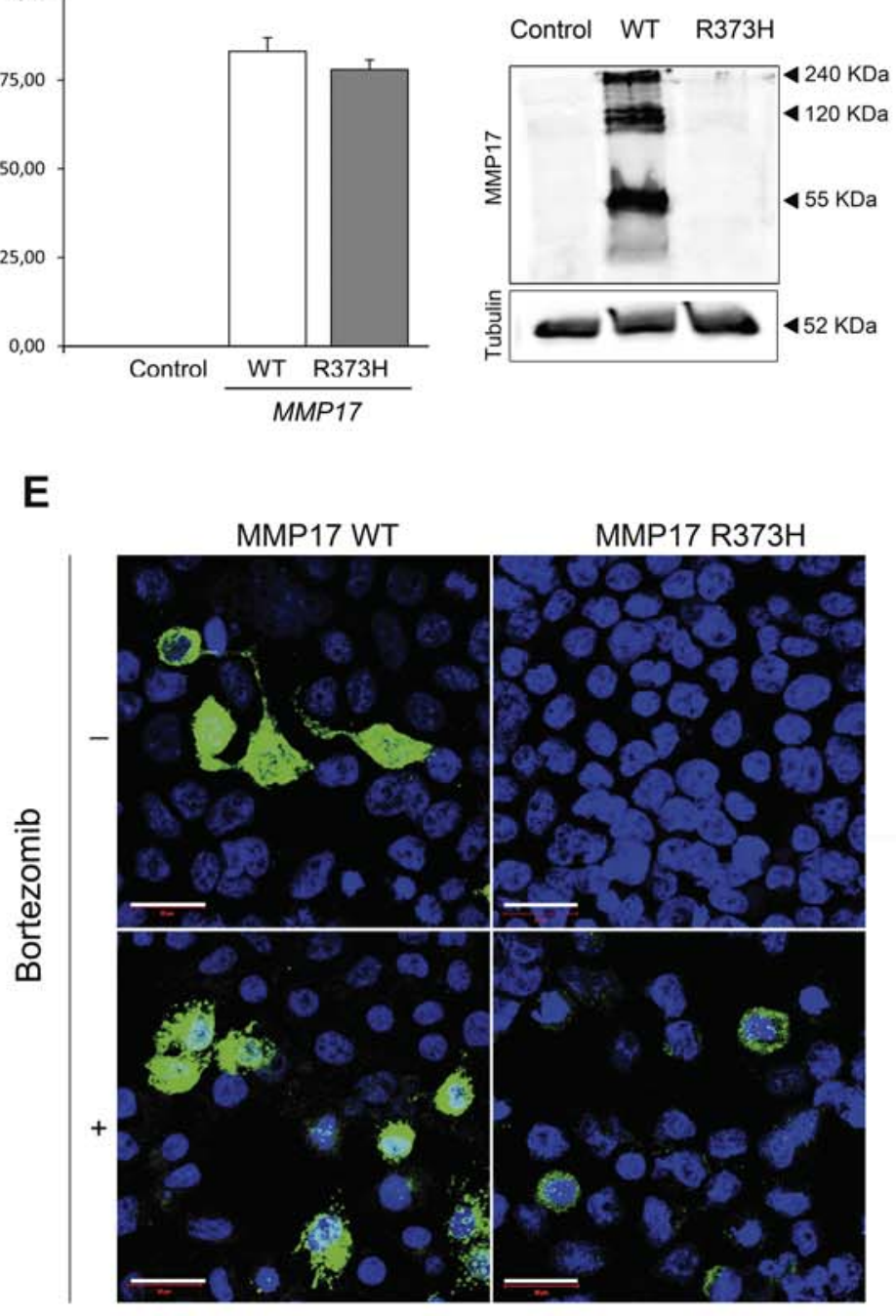

Figure 1-R3 

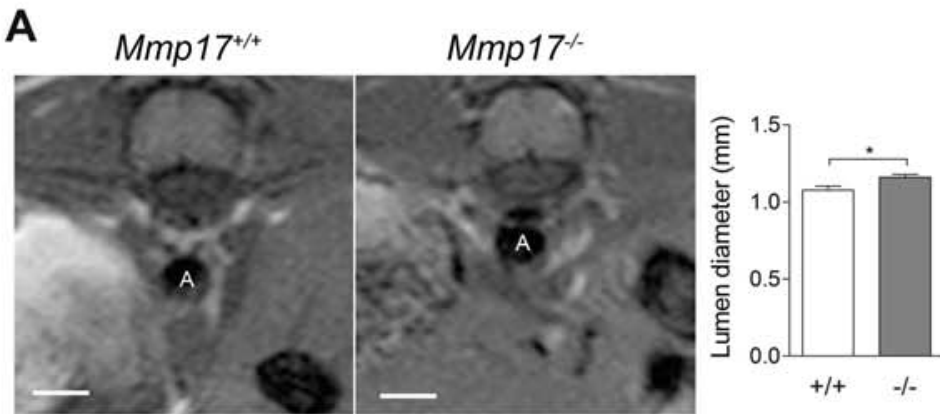

C

F

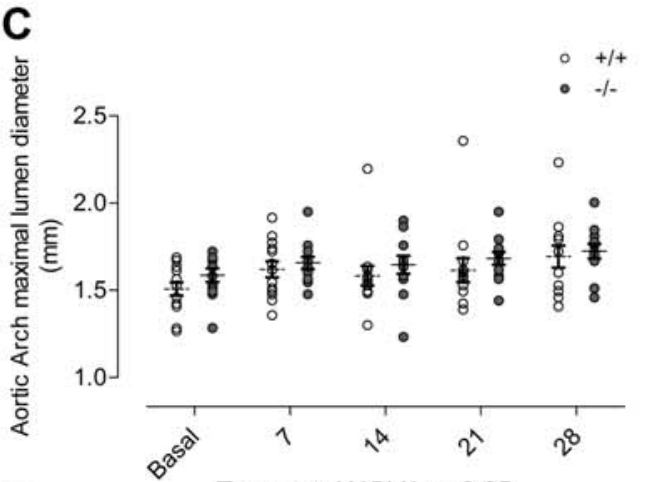

D

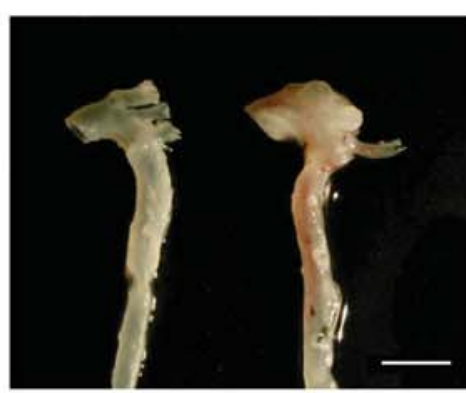

E

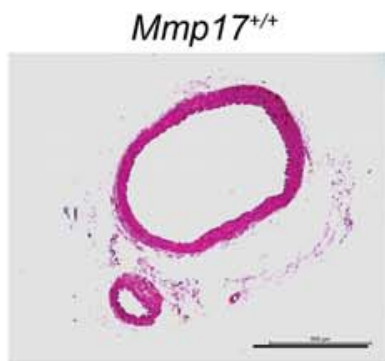

H

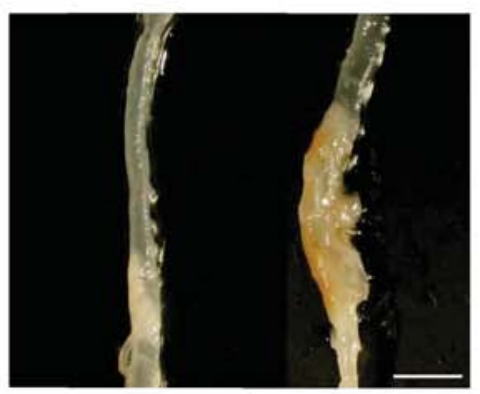

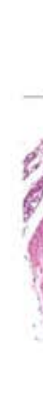

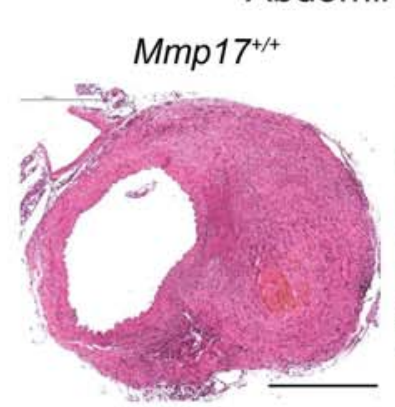

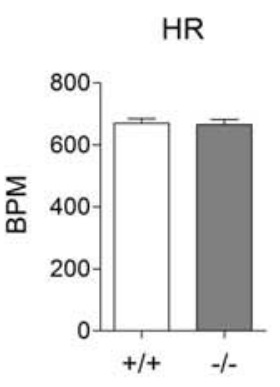

Aortic Arch

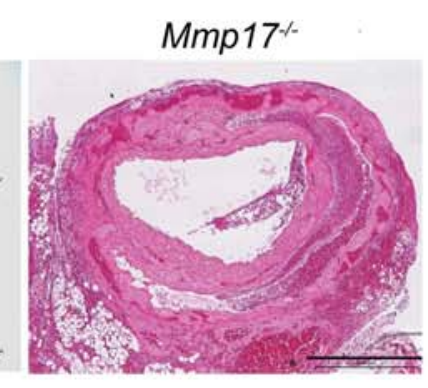

Abdominal Aorta

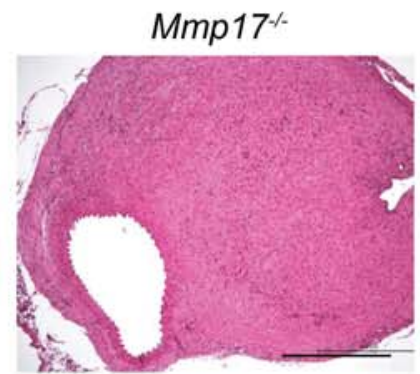

Figure 2-R3 
A
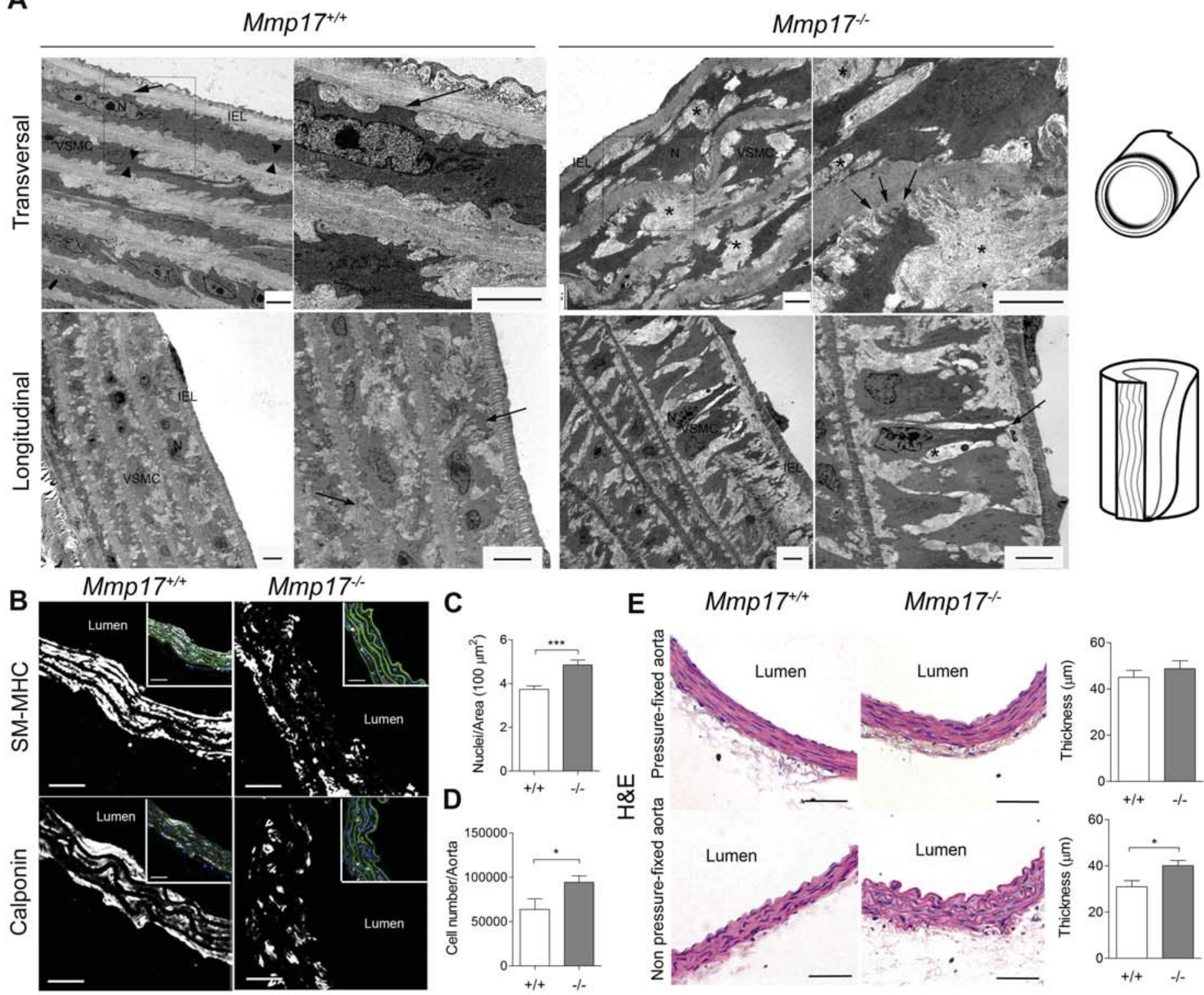

Mmp17\%
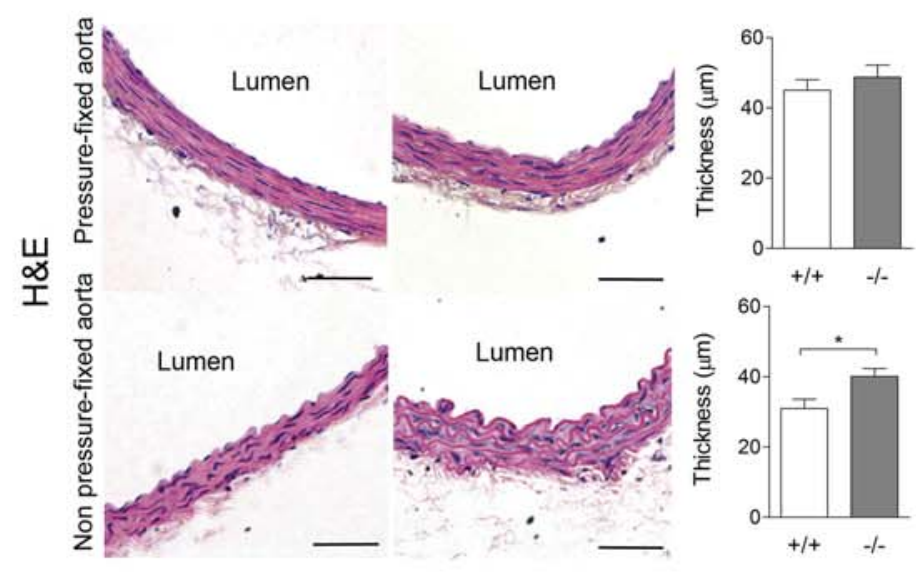

Figure 3-R3 
A $\quad M m p 17^{+/+}$

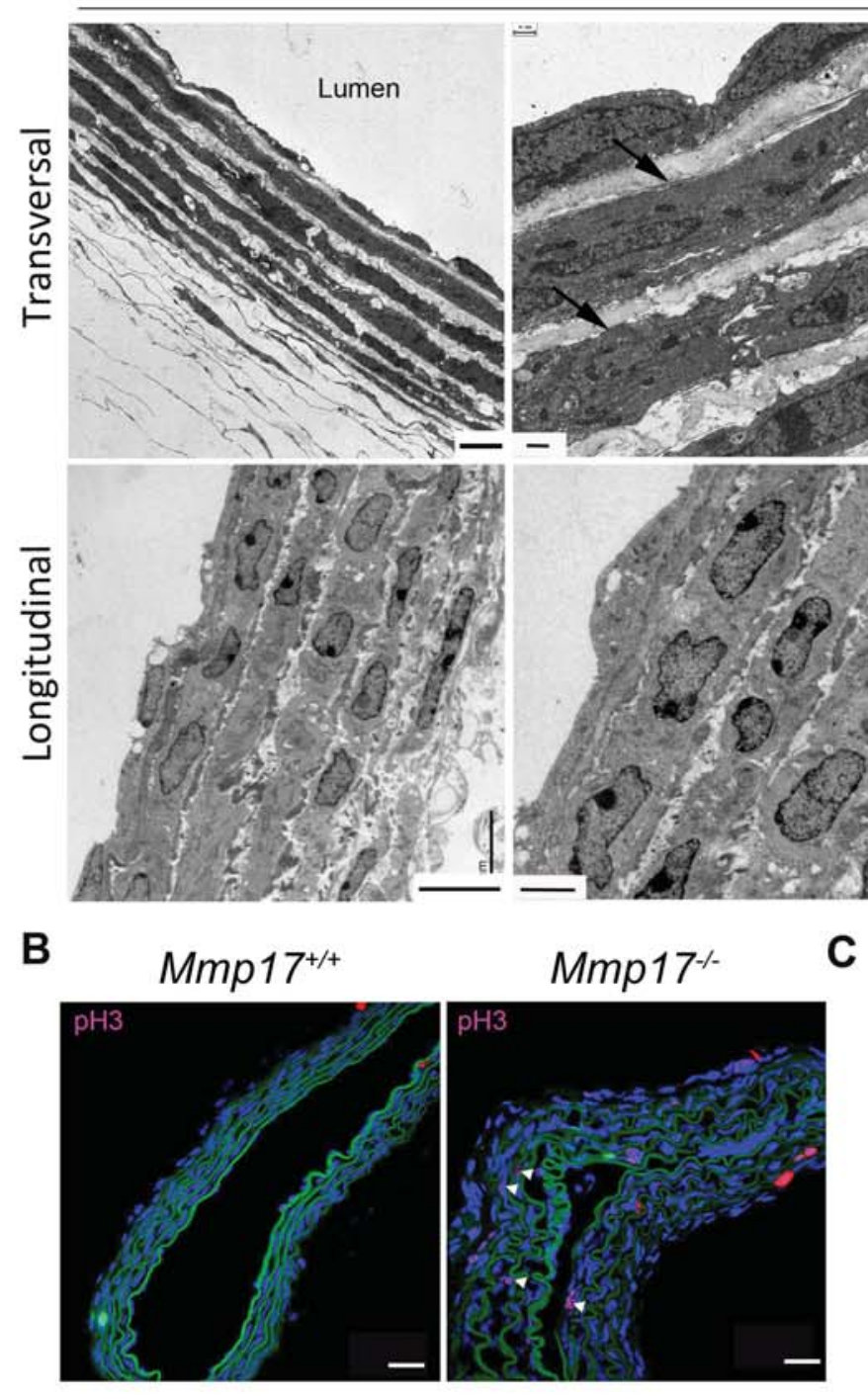

$M m p 17^{-/}$

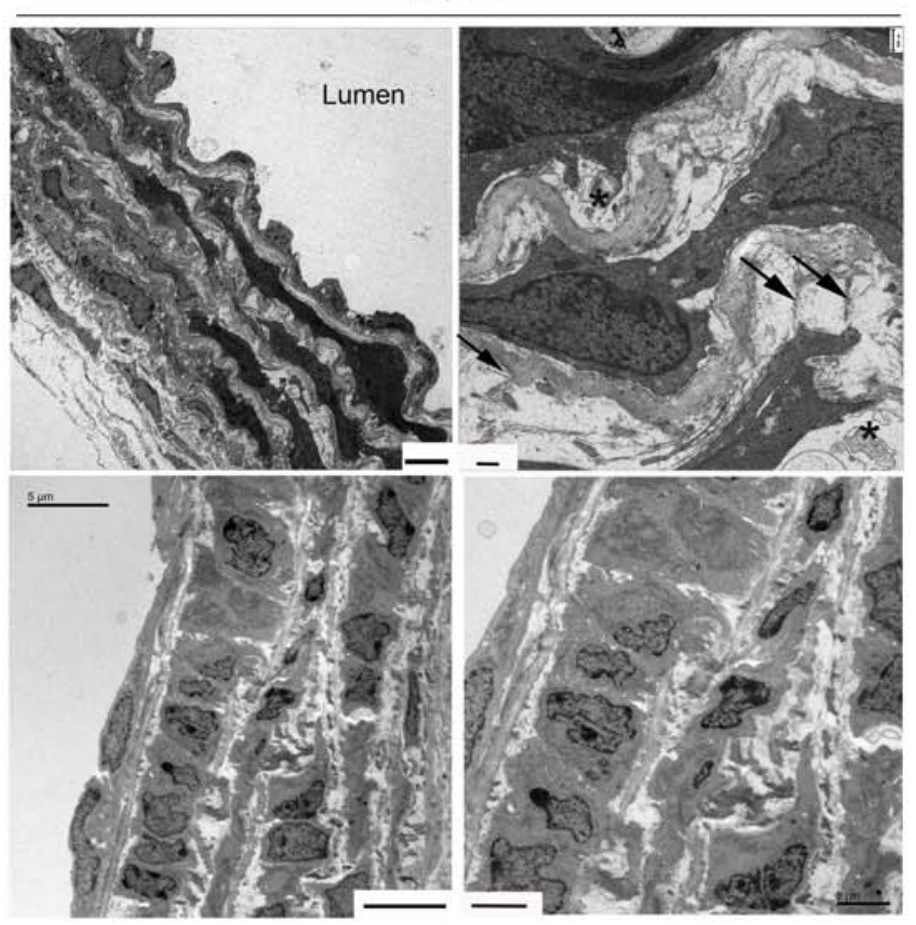

D

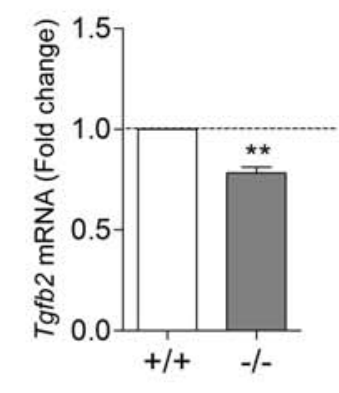

Figure 4-R3 
A

\section{Neonate}

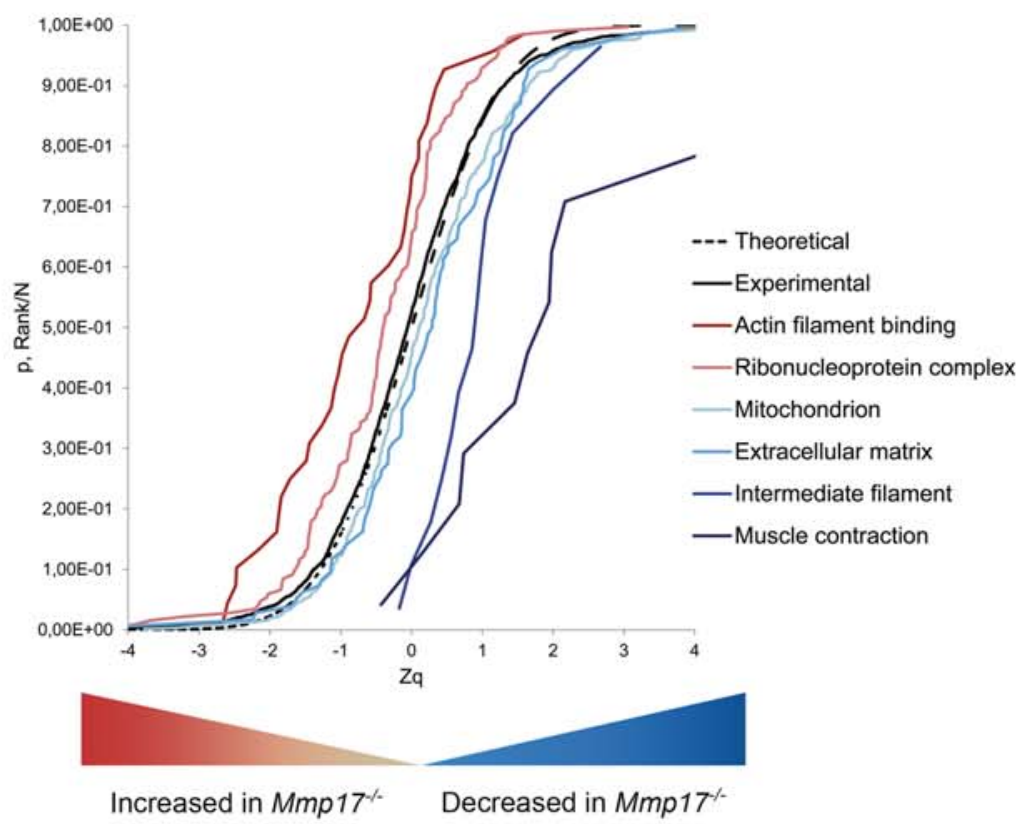

B

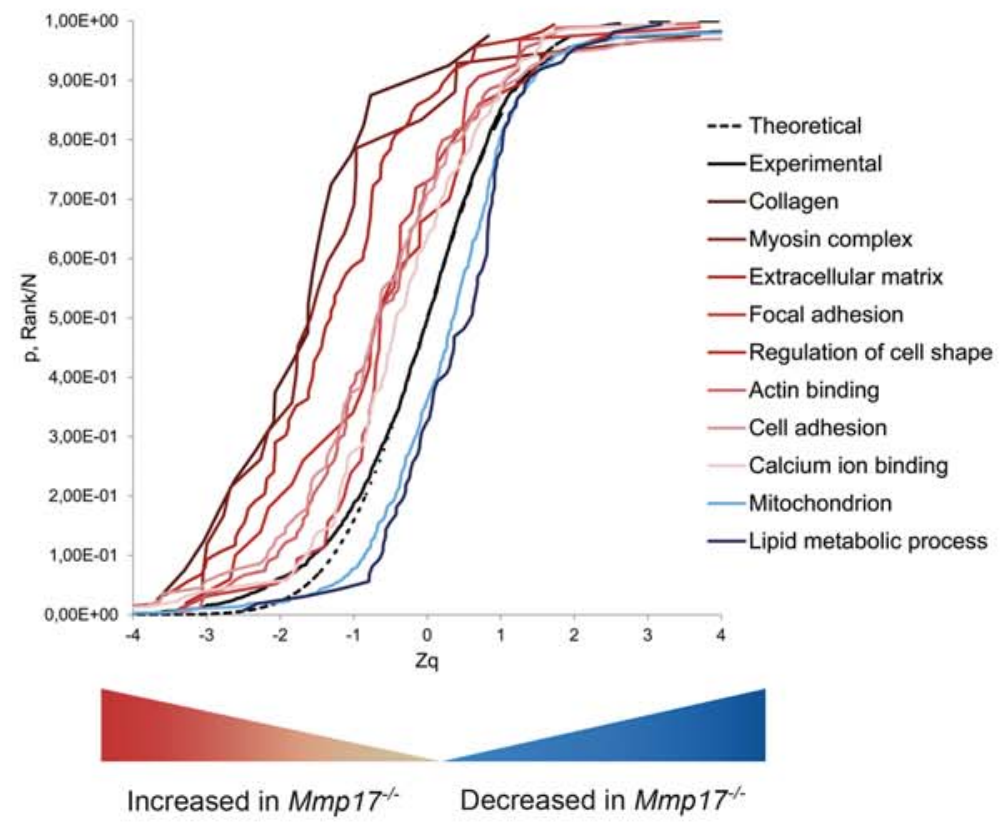

Figure 5R3 
A

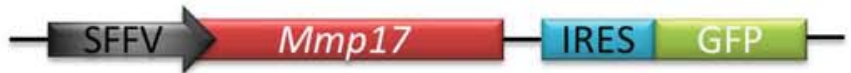

B

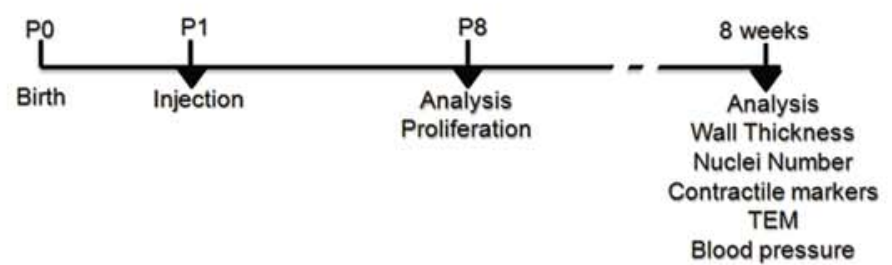

D

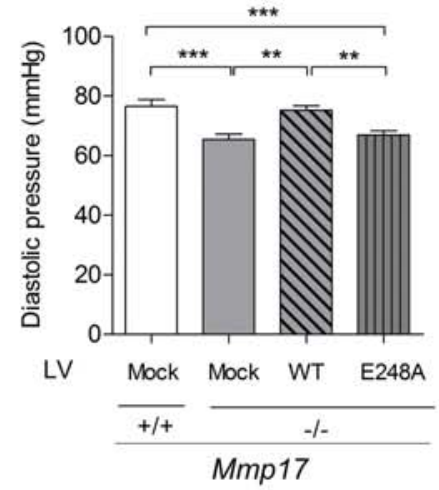

G

H

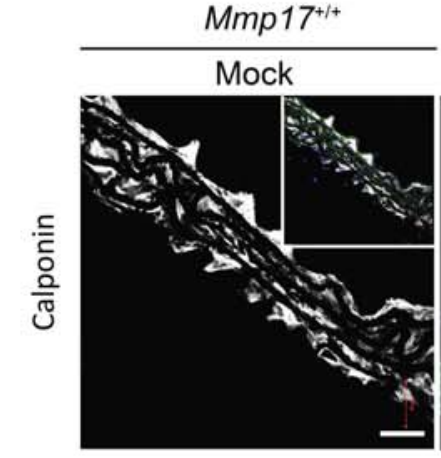

E

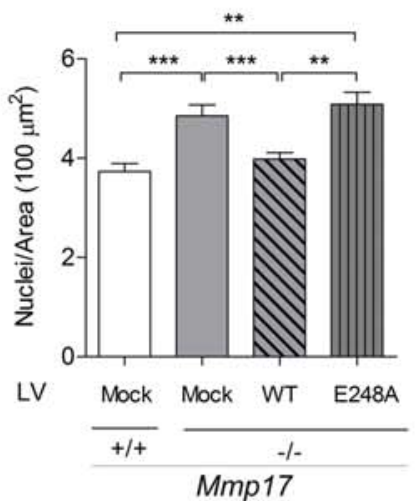

H

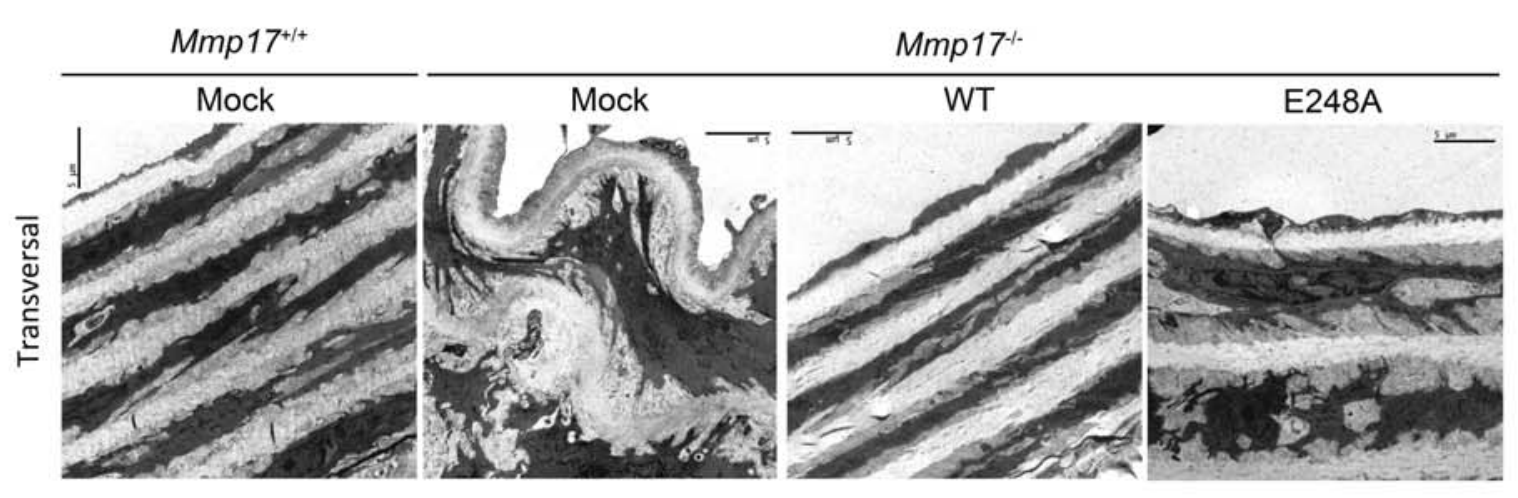

C

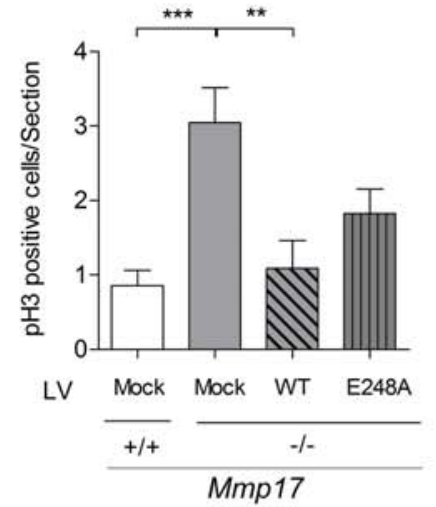

F

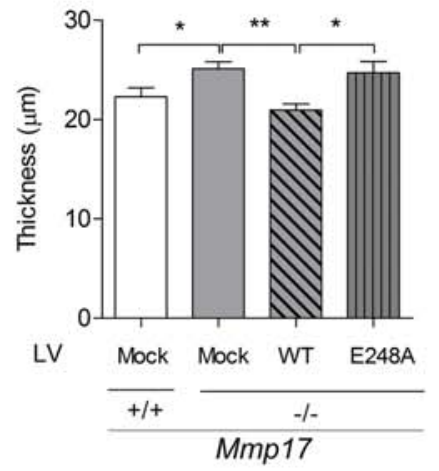

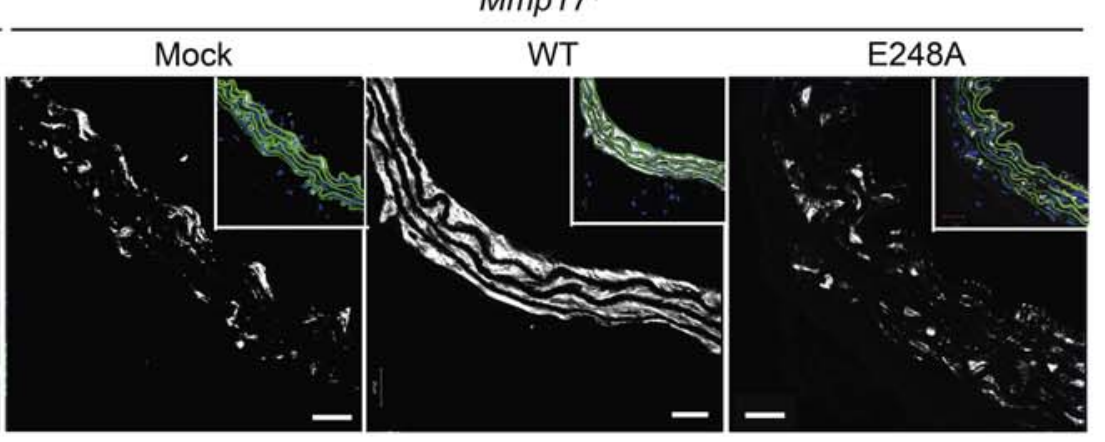


A

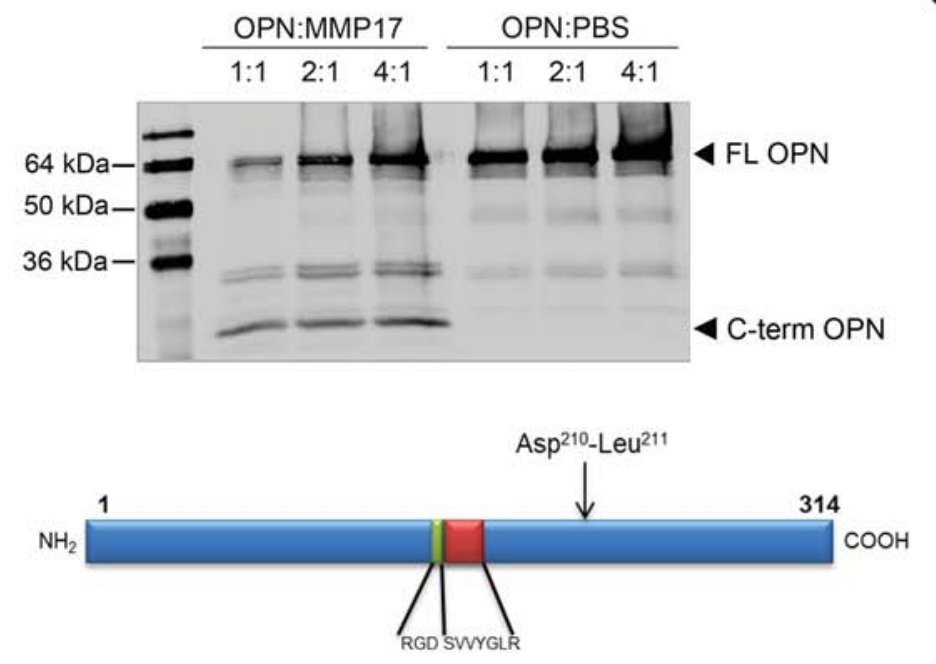

C

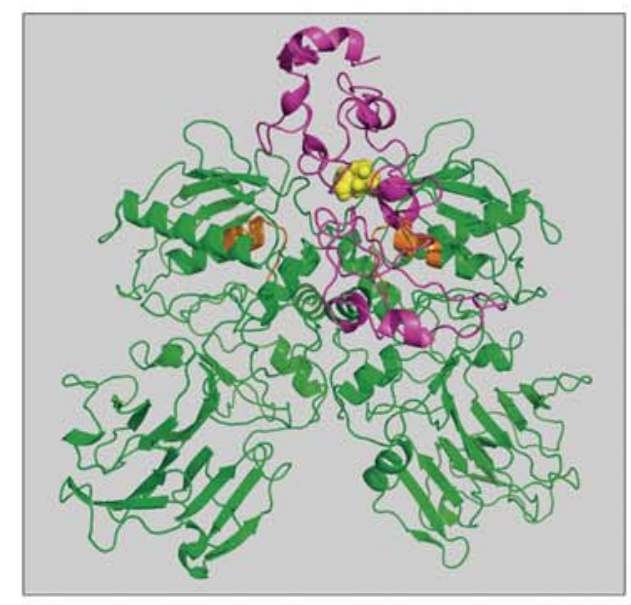

D

$M m p 17^{+/+}$

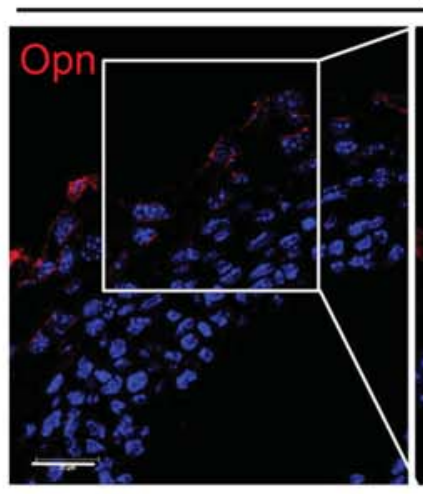

E

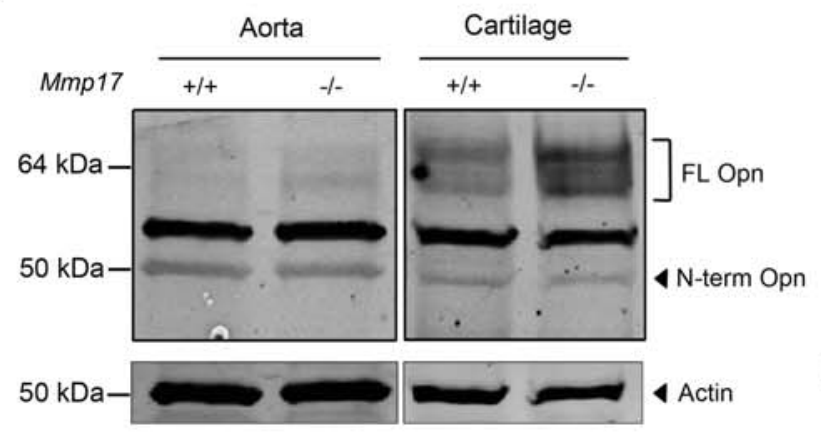

G
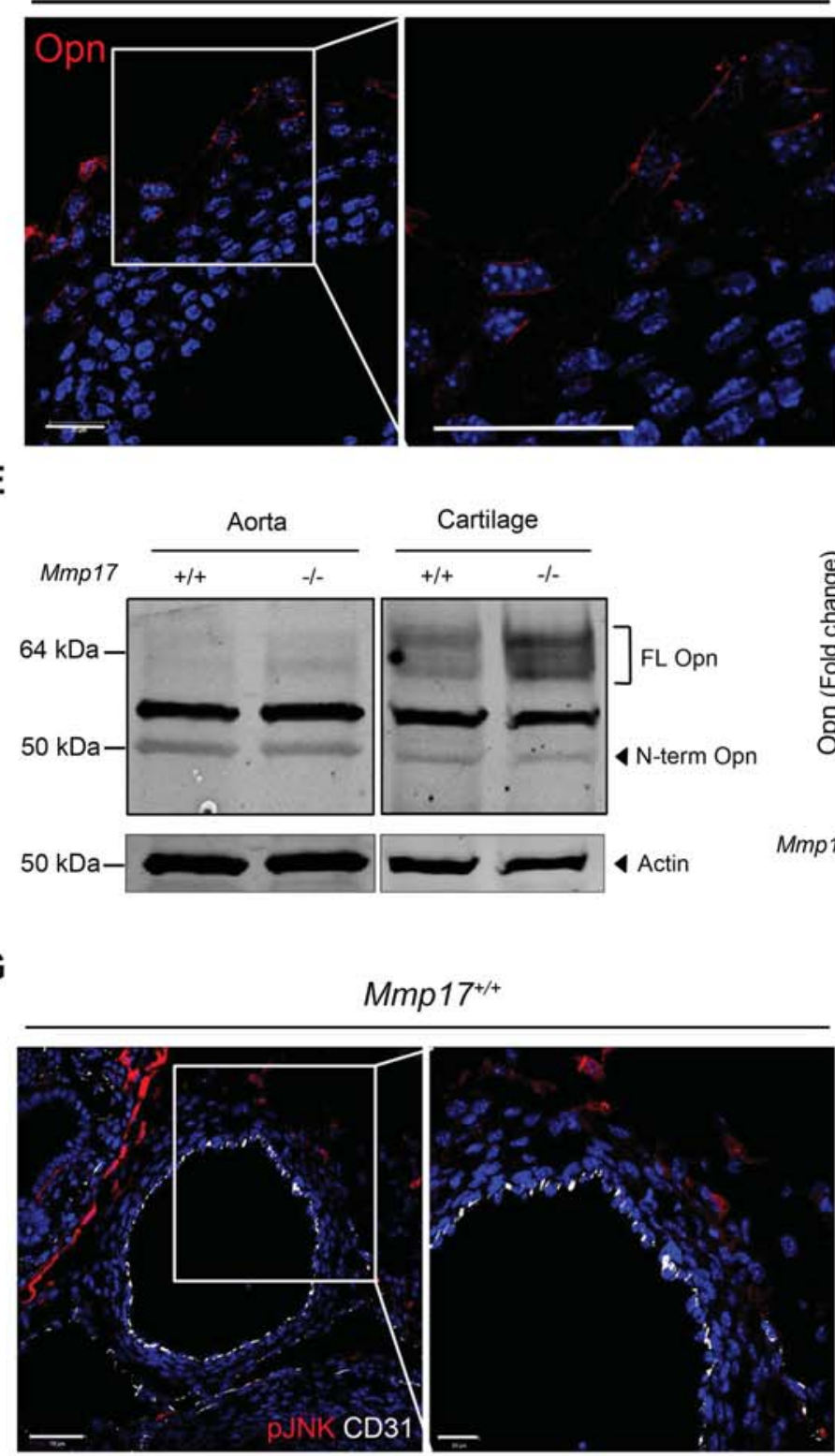
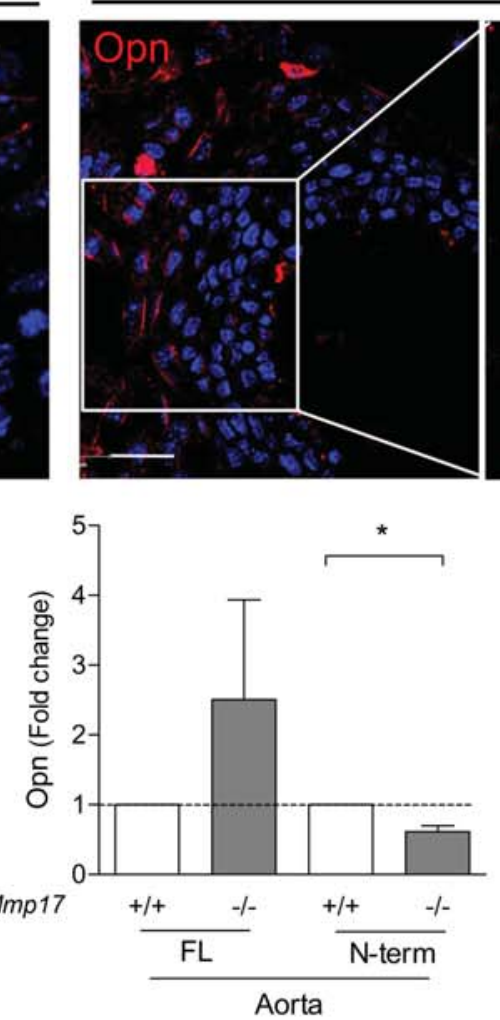

$M m p 17^{\%}$

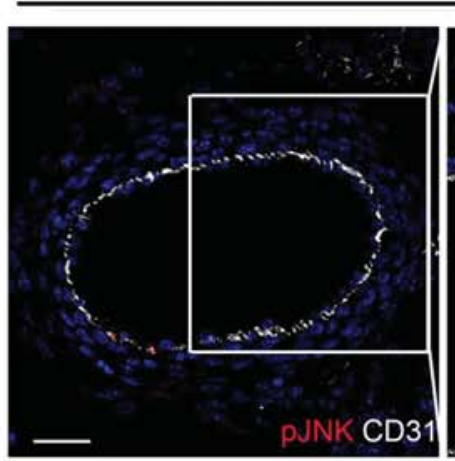

F

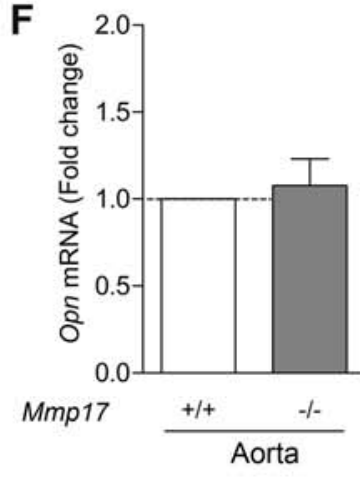

Mmp17
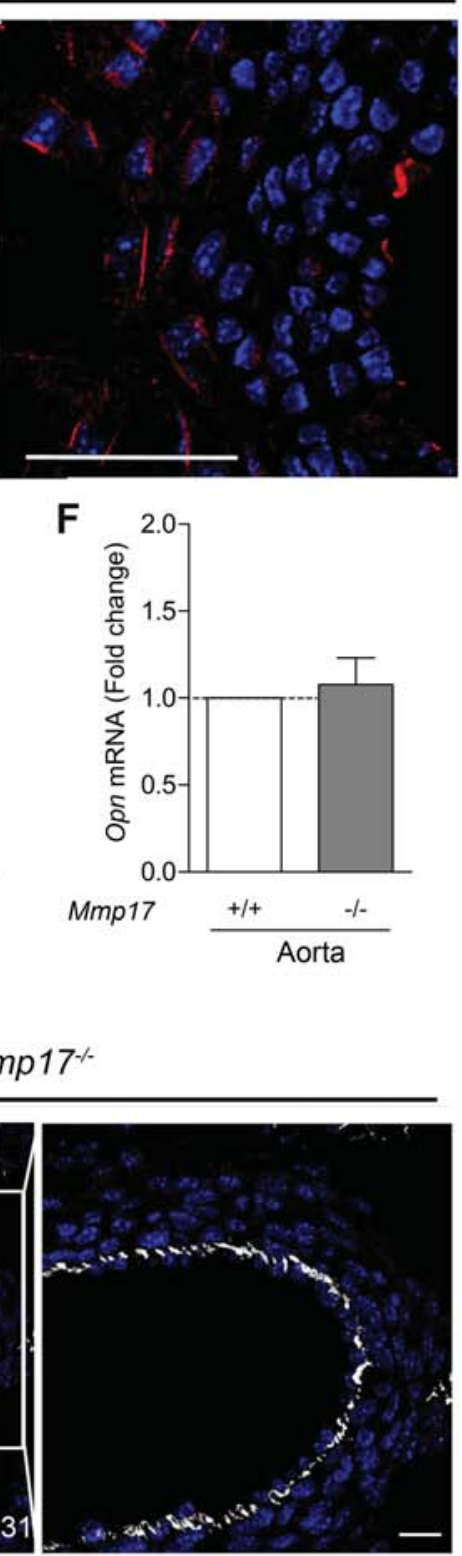

Figure 7-R3 
A

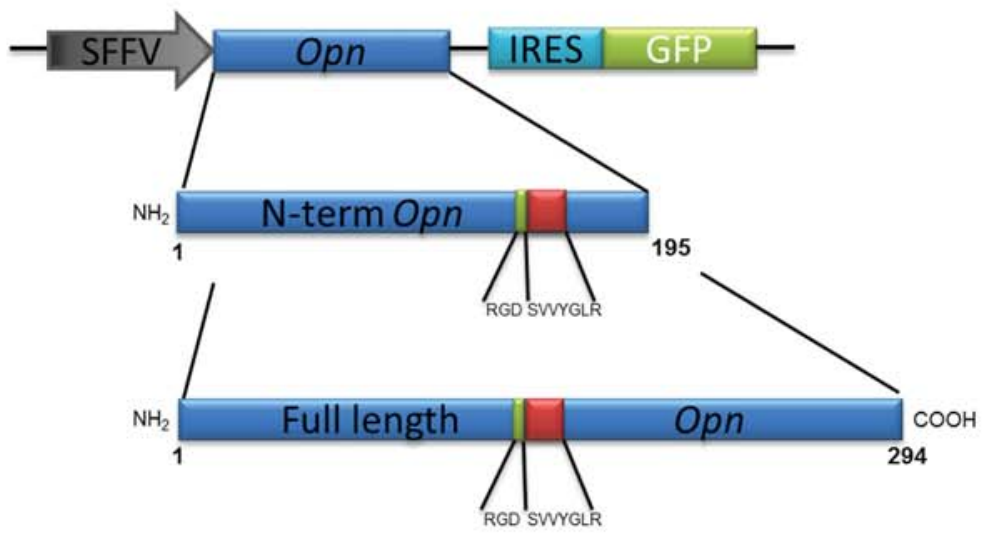

B

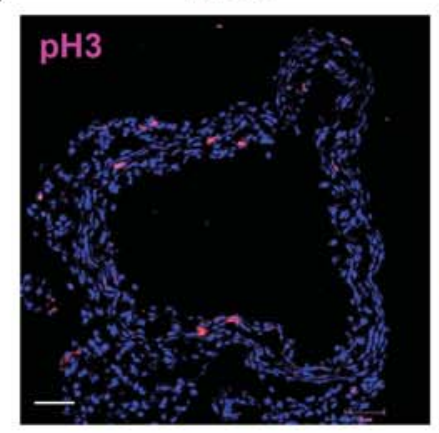

D

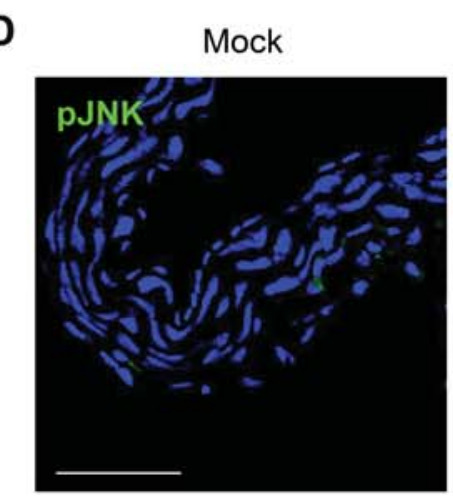

N-term Opn

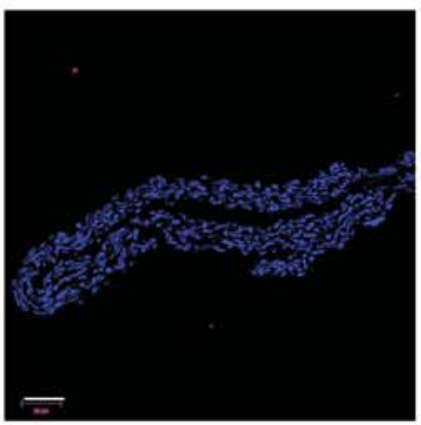

N-term Opn

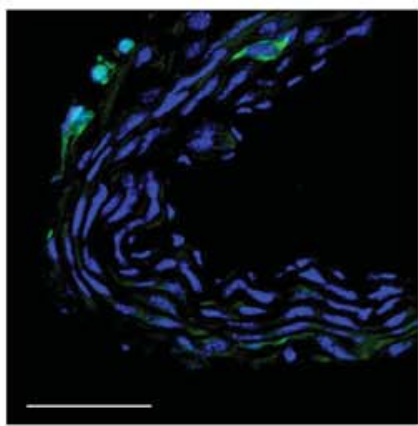

Full length Opn

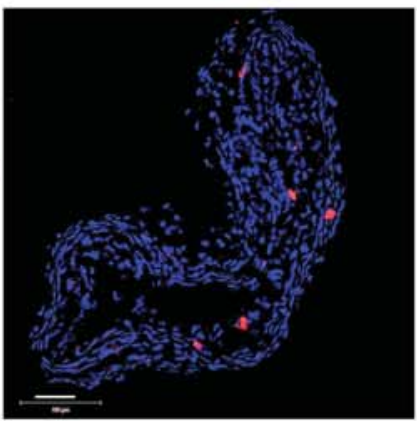

Full length Opn

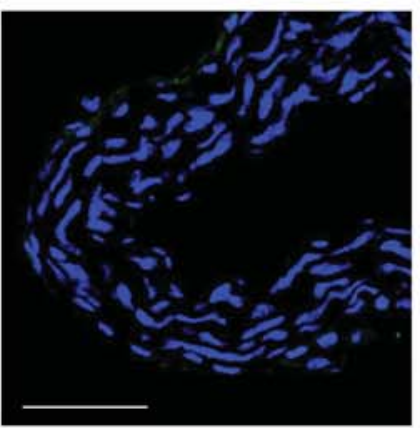

C

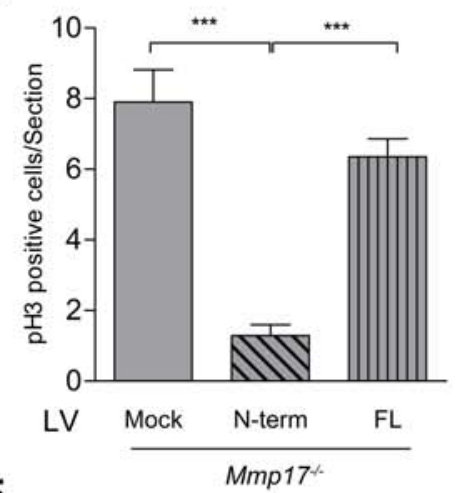

E

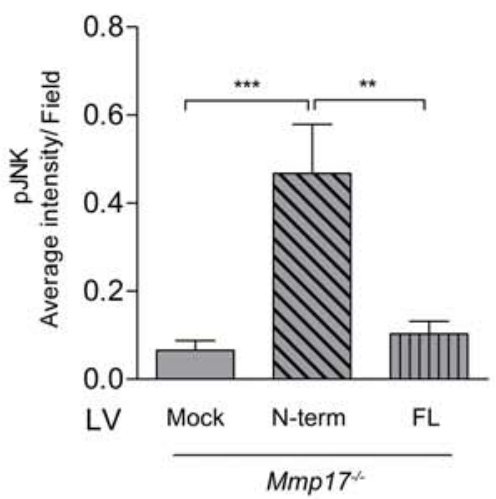

Figure 8-R3 


\section{Supplemental Material}

\section{Supplemental Methods}

Exome sequencing. The study protocol was approved by the Committee for the Protection of Human Subjects at the University of Texas Health Science Center at Houston, and the study participants gave informed consent. Genomic DNA was extracted from peripheral blood lymphocytes using standard protocols. Five $\mu$ g samples of DNA from probands were used for construction of the shotgun sequencing library using adaptors for paired-end sequencing. ${ }^{1}$ Exome sequences were captured with SeqCap EZ. Exome probes version 1.0 (Roche, Basilea, Switzerland) and recovered according to manufacturer's directions. Enriched libraries were then sequenced on an Illumina (San Diego, USA) GAIIx. Reads were mapped to the reference human genome (UCSC hg19, Santa Cruz, USA) with BWA (Burrows-Wheeler Aligner), ${ }^{2}$ and variants called with SAMtools. $^{3}$

MMP17 in silico modeling. A theoretical model of the MMP17 protein was calculated using the de novo prediction method with the structural homologs comparison approach. The Fasta sequence (603 aminoacids (aa)) of the MMP17 protein precursor (UniProtKB: Q9ULZ9.4) was processed to eliminate the signal peptide and pro-peptide sequences and yield a new sequence for the mature wild-type protein (440 aa: positions 126 to 565 of the precursor protein). A similar process was used with a point mutation (R373H) in the precursor protein sequence. Both MMP17 sequences were submitted to the iTASSER server (http://zhanglab.ccmb.med.umich.edu/I-TASSER/). ${ }^{4}$ We selected the PDB (Protein Data Bank) model with highest probability and minimal energy compatible with biochemical properties (di-S intrachain, accessible and exposed glycosylation sites and accessible active region [active site and neighboring zinc binding sites] C-score $=-0.43$ for wild-type (WT) and C-score $=-0.18$ for R373H-mutant). Both models were aligned, visualized and decorated using PyMol v1.6.0.0. In silico models of wild-type and R373H-mut MMP17 homodimers were calculated by submitting PDB models of monomeric MMP17 or monomeric MMP17-R373H to Hex server v 6.9 (http://hexserver.loria.fr/). ${ }^{5}$ Docking in dimer mode was predicted with shape and electrostatic restrictions to select the best (minimal energy) model of each 
dimer type compatible with biochemical properties (di-S interchain). ${ }^{6}$ Docking predictions for the MMP17 dimer (WT) as receptor and OPN as ligand were submitted to the ClusPro server (http://cluspro.bu.edu), ${ }^{7}$ and the best model compatible with the biological topology of the MMP17 binding site was selected. The model was then refined by closer pre-alignment of the MMP17 dimer to the binding site using PyMol v 1.6.0.0, and this refined preliminary model was submitted to the docking application of the ROSIE server (http://rosie.rosettacommons.org) with default values. From the ten models with the lowest energy, we selected the model with best topology (and smallest binding distance, score $=-770.875)$.

MMP17 R373H generation and HEK293 transfection. The human MMP17-PG6 vector was mutated with the QuikChange Site-Directed Mutagenesis Kit (Agilent Technologies, Santa Clara, USA) to yield the hMMP17 R373H mutant. The following primers were used: Fw 5'GGCACAGATGCACCACTTCTGGCGGG-3’ and Rv 5’CCCGCCAGAAGTGGTGCATCTGTGCC-3’. HEK293 cells (80\% confluence) were transfected over 3 hours with wild-type or R373H-mutated MMP17 vectors using Lipofectamine 2000 (Invitrogen, Waltham, USA), and expression was analyzed during the subsequent 24 hours. The transfected cells were treated with $100 \mu \mathrm{g} / \mathrm{ml}$ bortezomib (Sigma-Aldrich, St. Louis, USA) for 24 hours. Protein and RNA were extracted as described later. For immunofluorescence assay, HEK293 cells were fixed for 10 minutes in 4\% paraformaldehyde (PFA) in PBS and permeabilized with $0.1 \%$ Triton X-100 for 5 minutes. The anti-human MMP17 antibody (M3684, Sigma-Aldrich) was used at 1:100 dilution followed by secondary anti-rabbit antibody and cells were visualized under a Zeiss LSM 700 microscope. Figure panels show maximal projections of confocal images.

Mouse Line. $M m p 17^{/-}$mice in the C57BL/6 background have been described previously. ${ }^{8}$ Mice were handled under pathogen-free conditions in accordance with CNIC institutional guidelines. Experiments were performed in accordance with Spanish legislation on animal protection and were approved by the local governmental animal care committee. All mice were genotyped by PCR of tail samples using the following primers: Mmp17 SK1 5'-TCAGACACAGCCAGATCAGG-3' 
SK2 5'- AGCAACACGGCATCCACTAC-3' and SK3 5'-AATATGCGAAGTGGACCTGG-3'. Experiments were conducted on mice in three age groups: embryos (E10.5 to E18.5), neonates (P0 to P30), or adults (8- to16-weeks old).

Magnetic resonance imaging. Magnetic resonance images were obtained in anesthetized adult mice (12 weeks old) using a Bruker Biospec 47/40 instrument (60 mm gradient, 35 mm internal diameter quadrature coil). Images were taken every millimeter in the descending thoracic and

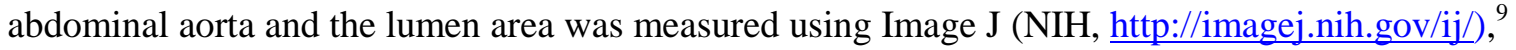
and OsiriX software (Geneva, Switzerland).

Hemodynamic measurements. Tail cuff measurements of blood pressure were taken in groups of 10 mice per genotype and condition, using BP2000 (Visitech Systems, Apex, USA). Ten recordings of each conscious mouse were obtained after one week of training over 5 consecutive days at the same hour of the day. Average values were calculated for each mouse from the measurements recorded over the 5 days.

Angiotensin II infusion by miniosmotic pump implantation. 16-week-old mice were administered with angiotensin II by subcutaneous osmotic minipump infusion (Alzet DURECT Corporation, Cupertino, USA) as described previously. ${ }^{10}$ Angiotensin II (Sigma-Aldrich) was administrated at $1 \mu \mathrm{g} / \mathrm{kg} / \mathrm{min}$ during 28 days and blood pressure was monitored once a week by tail cuff. Aortic lumen diameter of ascending thoracic aorta and abdominal aorta was analyzed by high-frequency ultrasound with the VEVO770 system (VisualSonics, Toronto, Canada). TAA was defined as $\geq 25 \%$ increase in aortic arch intraluminal diameter together with the presence of the following histological features: breakage and loss of elastin fibers, inflammatory infiltrate in the adventitia and/or media, VSMC loss, and thickening of the media on one side and thinning on the opposite site of the aortic section. ${ }^{11}$ AAA was defined by intraluminal diameter of the abdominal aorta (suprarenal area) $\geq 1.3 \mathrm{~mm}$ together with the presence of 
pronounced ECM remodeling and thrombus. ${ }^{12}$ After perfusion with 4\% PFA and 2\% sucrose in PBS, aortas were embedded in paraffin for histological analysis.

Histological, LacZ and immunohistochemical studies. Aortas were excised after perfusion under constant pressure with 4\% PFA, 2\% sucrose in PBS (pressure-fixed aortas) or perfusion with PBS alone (non-pressure-fixed aortas). Aortas were then either embedded directly in OCT or further fixed for 12 hours and embedded in paraffin. Paraffin-embedded aorta cross-sections $(7 \mu \mathrm{m})$ were stained with H\&E, picrosirius red, alcian blue or Verhoeff-van Gieson stain. Aortic medial thickness, density of nucleus, lumen area, and elastin lamellae number were measured independently by two researchers blinded to sample identity, and were quantified using MetaMorph software (Molecular Devices LLC. Sunnyvale, California). Endothelial and adventitial cells were excluded from the analysis. For LacZ analysis, OCT frozen sections (5 $\mu \mathrm{m})$ of vessels and embryos were fixed in $0.125 \%$ glutaraldehyde in PBS for 5 minutes, rinsed in PBS and incubated at $37^{\circ} \mathrm{C}$ overnight in X-gal buffer ( $5 \mathrm{mM}$ potassium ferrocyanide, $5 \mathrm{mM}$ potassium ferricyanate, $2 \mathrm{mM} \mathrm{MgCl}_{2}$ and 0.1\% X-gal in PBS). Immunohistochemical staining for Mmp17 (PAB4785, Abnova, Taipei, Taiwan) was performed on OCT sections fixed in 4\% PFA, 2\% sucrose for 7 minutes; for SM-MHC (BT-562, Biomedical Technologies Inc., Alfa Aesar, Ward Hill, USA), calponin (E798Y clone, ab46794, Abcam, Cambridge, UK), and anti- pH3 (06-570, Millipore, Billerica, USA), OCT sections were first permeabilized with $0.1 \%$ Triton X-100 for 5 minutes. Sections were then processed for incubation with specific secondary antibody and Hoechst stain. pH3-positive medial aortic cells were quantified by counting the number of positive VSMCs per transverse section. One to five sections were analyzed per mouse. For GFP analysis, overnight fixed (4\% PFA) aortas were embedded in OCT and sections were stained with anti-GFP antibody (ab13970, Abcam). For analysis of aortas in embryos, E14.5 embryos were perfused and fixed overnight in 4\% PFA, 2\% sucrose solution followed by 30\% sucrose solution for 3 hours. OCT sections were stained for Opn (AF808 polyclonal antibody, R\&D, Minneapolis, USA), $\beta$ galactosidase (ab4761, Abcam), phospho-JNK (9255, Cell signaling, Danvers, USA) and CD31 (MAB1398Z, Millipore) followed by the specific secondary antibody (Alexa Fluor, Invitrogen). 
Preparations were mounted in Fluoromount G imaging medium (4958-02, Affymetrix eBioscience, San Diego, USA). Images were acquired with Zeiss LSM 700 confocal microscope at $21-23^{\circ} \mathrm{C}$ using 25X (LD LCI Plan Apo 25X/0.8) and 63X (Plan Apo 63X/1.4 oil DICII) objectives. Images were analyzed using Zen 2011 software (Zeiss, Jena, Germany) and maximal projections are shown (25X magnification). Quantification of phospho-JNK was performed using MetaMorph software.

\section{RNA purification and quantitative reverse transcriptase PCR (Q-RT-PCR). Total RNA} was isolated from E14.5 embryonic aortas, neonate or adult descending aortas, or cultured cells with the RNeasy® Mini Kit (Qiagen, Venlo, Netherlands). Aortas were first placed in lysis buffer and disrupted with sterile ceramic beads using a MagNA Lyser (Roche), with two rounds of $6500 \mathrm{rpm}$ for 30 seconds each, with care taken to maintain the sample cold. RNA was quantified by spectrophotometry (ND1000 Spectrophotometer, NanoDrop, (Thermo Scientific, Wilmington, USA)) and RNA integrity was monitored by ethidium bromide staining after agarose gel electrophoresis. cDNA was synthetized from $1 \mu \mathrm{g}$ of total RNA using the Qiagen Omniscript RT Kit (Qiagen). Q-RT-PCR was performed using TaqMan probes for mouse Mmp17 (Mm00449292), human MMP17 (Hs00211734) and mouse Tgfb2 (Mm00436955) in an ABI PRISM ${ }^{\circledR} 7900 H T$ FAST Q-RT-PCR System. All samples were analyzed in triplicate, and RNA levels (CNRQ; calibrated normalized relative quantity) were obtained with Biogazelle qBase PLUS software. For human analysis, hTFRC (00951083_m1), hHMBS (00609297) and hTBP (00427621) housekeeping genes were used. For mouse analysis, mGapdh (99999915), mTbp (00446973) and mHprt (00446968) probes were used for normalization (Taqman). SYBR green (Applied Biosystems, Carlsbad, USA) was used in mOpn mRNA analysis. mOpn probes were as follow: FW 5'-AGCAAGAAACTCTTCCAAGCAA-3'; RV 5'GTGAGATTCGTCAGATTCATCCG-3'. mGapdh and mTbp were used for normalization (mGapdh: FW 5’-AATGCATCCTGCACCACCAA-3'; RV 5’GTGGCAGTGATGGCATGGA-3' and mTbp: FW 5'-GCTCTGGAATTGTACCGCAG-3'; RV 5'-CTGGCTCATAGCTCTTGGCTC-3’). 
Protein extraction and western-blot analysis. Protein extracts were obtained from cell cultures as described. ${ }^{6}$ Briefly, cell monolayers were covered with cold lysis buffer (25 mM Tris- $\mathrm{HCl}$ [pH 8.0], $100 \mathrm{mM} \mathrm{NaCl}, 1 \%$ IGEPAL CA-630 and $60 \mathrm{mM}$ Octyl glucoside) supplemented with $20 \mathrm{mM}$ N-ethylmaleimide (NEM, Sigma-Aldrich) and protease inhibitors (Complete, Mini, EDTA-free, Roche). Descending aortas were disrupted with MagNA Lyser (Roche) in cold regular RIPA buffer or the specific Tris-based lysis buffer described above. Proteins (50 to $150 \mu \mathrm{g}$ ) were resolved by 712\% SDS-PAGE in reducing or non-reducing conditions and transferred onto nitrocellulose membranes (0.45 $\mu \mathrm{m}$, Bio-Rad, Hercules, California). Antibodies against MMP17 (EP1270Y clone, ab51075, Abcam,), tubulin (ab6160, Abcam,) and $\beta$-actin (A5441, Sigma-Aldrich,) were used at 1:1000 dilution. For Opn analysis in E14.5 embryos a pool of 8-to-10 aortas or a piece of cartilage were disrupted as before and resolved on 4-20\% gradient gels (Bio-Rad). Antibodies to Opn (18621, IBL, Fujioka, Japan) and calponin (E798Y clone, ab46794, Abcam) were used at a 1:1000 dilution overnight. Immunoreactive proteins were visualized with corresponding fluorochrome-conjugated secondary antibodies (680 or 800 ODYSSEY IRDye ${ }^{\circledR}$ ) and recorded by Licor Odyssey technology. Quantification of western-blot bands was performed using ODYSSEY software (LI-COR Biosciences, Lincoln, USA).

Transmission electron microscopy. Male mice were anesthetized and trans-cardially perfused with Sörensen solution [pH 7.3] at $100 \mathrm{mmHg}$ pressure (50 ml) followed by fixation solution (50 ml PBS containing 4\% PFA and 1\% glutaraldehyde) using a peristaltic pump (Dynamax, Rainin RP1, Mettler Toledo, Greifensee, Switzerland). Dissected arteries were additionally fixed in the same solution for 16 hours. Arteries were then embedded in resin (Durcupan ACM Fluka, SigmaAldrich) for transverse or longitudinal sectioning, and ultrathin sections were cut and counterstained by the Electron Microscopy core (SIdI service) at the Universidad Autónoma de Madrid (Madrid). Sections were imaged using a transmission electron microscope (Jeol Jem 1010, 80Kv, Jeol Ltd. Tokyo, Japan) and recorded with a Gatan camera (Orius, SC200, Pleasenton, California) at the indicated magnifications. VSMC phenotype was determined according to reported criteria. ${ }^{13}$ Thoracic descending aortas and mesenteric arteries of adults or neonatal mice of 
each genotype and condition were visualized and used for morphological and ultrastructural analysis and for quantification of medial thickness with MetaMorph software.

VSMC isolation and culture. Six to eight aortas from 8-week-old male mice were pooled to obtain a cell suspension. ${ }^{14}$ Briefly, after fat removal under a microscope, aortas were incubated in collagenase solution (collagenase type I, $3.33 \mathrm{mg} / \mathrm{ml}$, Worthington, Lakewood, USA) for 5 minutes at $37^{\circ} \mathrm{C}$, allowing the removal of the adventitia with forceps. The aortas were then cut into small pieces and a cell suspension was obtained by incubation for 45 minutes at $37^{\circ} \mathrm{C}$ in $6 \mathrm{mg} / \mathrm{ml}$ type I collagenase and $2.5 \mathrm{mg} / \mathrm{ml}$ elastase (Worthington) diluted in DMEM. VSMCs were counted in a Neubauer chamber and cultured on 0.02\% gelatin-coated plates in DMEM supplemented with 20\% fetal bovine serum (FBS) (Gibco, Invitrogen), 2 mM L-glutamine (Lonza, BioWhittaker, Basel, Switzerland), $0.1 \mathrm{mM}$ nonessential amino acids (Sigma-Aldrich) and penicillin (50 UI/ml), streptomycin $(50 \mu \mathrm{g} / \mathrm{ml})(\mathrm{P} / \mathrm{S})$ (Lonza, BioWhittaker). Endothelial cells (mouse aortic endothelial cells, MAEC) were positively selected from the VSMC culture with an antibody to intercellular adhesion molecule 2 (anti-ICAM-2; 553325 BD Biosciences, Franklin Lakes, USA) coupled to magnetic beads, and were cultured as indicated. ${ }^{15}$ Macrophages were differentiated from bone marrow cells by treatment for 7 days with interleukin-3 (IL-3, PeproTech, Rocky Hill, USA), granulocyte macrophage colony-stimulating factor (GM-CSF, PeproTech) and stem cell factor (SCF, PeproTech) (20 ng/ml) in IMDM medium (Lonza) supplemented with 10\% FBS, followed by incubation for 5 days with M-CSF (PeproTech) $(10 \mathrm{ng} / \mathrm{ml})$ in the same medium. VSMC morphology was analyzed at passage 0 by fixing cultures in 4\% PFA, 2\% sucrose for 10 minutes and staining with Texas Red ${ }^{\circledR}$-X-phalloidin (1:200; Invitrogen). Total cell area and shape factor or roundness $\left(4 \pi\right.$ Area/Perimeter $\left.{ }^{2}\right)$ were calculated by masking with MetaMorph software.

Lentiviruses encoding GFP (Mock), N-term Opn or full-length Opn (see below) were incubated for 5 hours at 1-10 MOI (multiplicity of infection) with cultured VSMC (passage 0). After virus removal, GFP signal was analyzed at $24 \mathrm{~h}$ and $48 \mathrm{~h}$ (maximum of protein generation) after infection. Cells were fixed $48 \mathrm{~h}$ after virus infection in 4\% PFA for 10 min and washed with PBS. VSMC area and shape factor were evaluated in GFP-positive cells by analysis of confocal maximal 
projections (Zeiss LSM 700 confocal microscope) with Image J (NIH, http://imagej.nih.gov/ij/) ${ }^{9}$. Shape factor (or roundness) values close to 1 indicate a circular form, whereas values closer to 0 indicate an elongated object.

Myography experiments. The structural and mechanical properties of mesenteric resistance arteries were studied with a pressure myograph (Danish Myo Tech, Model P100, J.P. Trading I/S, Aarhus, Denmark). ${ }^{16}$ Briefly, the vessel was placed on two glass microcannulae and secured with a nylon surgical suture. After tying off any small branches, vessel length was adjusted so that the vessel walls were parallel without stretch. Intraluminal pressure was then raised to $120 \mathrm{mmHg}$ and the artery was unbuckled by adjusting the cannula. The segment was then set to a pressure of 70 mmHg and allowed to equilibrate for 30 minutes at $37^{\circ} \mathrm{C}$ in calcium-free $\mathrm{KHS}\left(0 \mathrm{Ca}^{2+}-\mathrm{KHS}\right.$, prepared by omitting calcium and adding 10 mM EGTA). Intraluminal pressure was reduced to 3 mmHg. A pressure-diameter curve was obtained by increasing intraluminal pressure in $20 \mathrm{mmHg}$ steps between 3 and $120 \mathrm{mmHg}$. Internal and external diameters were continuously measured under passive conditions $\left(\mathrm{D}_{\mathrm{i} 0 \mathrm{Ca}}, \mathrm{D}_{\mathrm{e} 0 \mathrm{Ca}}\right)$ for 3 minutes at each intraluminal pressure. The final value used was the mean of the measurements taken during the last 30 seconds, when the measurements reached a steady state. Finally, the artery was set to $70 \mathrm{mmHg}$ in $0 \mathrm{Ca}^{2+}-\mathrm{KHS}$, pressure-fixed with 4\% PFA in $0.2 \mathrm{mM}$ phosphate buffer [pH 7.2-7.4] at $37^{\circ} \mathrm{C}$ for 60 minutes, and kept in $4 \%$ PFA at $4^{\circ} \mathrm{C}$ for confocal microscopy studies. Passive structural and mechanical parameters were calculated from internal and external diameter measurements in passive conditions as follows: Wall thickness, $\mathrm{WT}=\left(\mathrm{D}_{\mathrm{e} 0 \mathrm{Ca}}-\mathrm{D}_{\mathrm{i} 0 \mathrm{Ca}}\right) / 2$ and Circumferential wall stress $\left(\left(\sigma=\left(\mathrm{P} \times D_{\mathrm{i} 0 \mathrm{Ca}}\right) /(2 \mathrm{WT})\right.\right.$, where $\mathrm{P}$ is the intraluminal pressure $\left(1 \mathrm{mmHg}=1.334 \times 10^{3}\right.$ dynes $\left./ \mathrm{cm}^{2}\right)$ and WT is wall thickness at each intraluminal pressure in $0 \mathrm{Ca}^{2+}$-KHS. The content and organization of elastin and the number of VSMCs in mesenteric arteries were studied in pressure-fixed $(70 \mathrm{mmHg})$, maximally relaxed intact segments using a confocal microscope. ${ }^{16}$ Briefly, stacks of images from the adventitia to the lumen (z step $=0.5 \mu \mathrm{m}$ ) were captured using the $488-\mathrm{nm}$ line of the confocal microscope. A minimum of 2 image stacks from different regions was captured in each arterial segment. All the images were taken under identical conditions of laser intensity, brightness and contrast. Quantitative analysis 
was performed with MetaMorph image analysis software. ${ }^{16}$ Internal elastic lamina (IEL) thickness was measured from each stack of serial images. Thereafter, individual projections of the IEL were reconstructed and total fenestrae area was measured.

Second harmonic generation multi-photon microscopy. Autofluorescence of organized collagen (425-465 nm filter) and elastin auto-fluorescence (478-573 nm filter) was visualized in fresh aortic sections from wild-type or $M m p 17^{\wedge}$ adult mice with a Zeiss LSM 780 microscope (Spectra-Physics Mai Tai DS [pulse $<70$ ps] Laser). 3D rendering reconstruction of aorta thickness (Z stack between 35 and $54 \mu \mathrm{m}$ ) was performed using Imaris software (Bitplane, Switzerland).

Carotid artery ligation. The left carotid artery was ligated as described previously. ${ }^{17}$ Twenty-eight days later, mice were anesthetized and perfused with PBS (5 ml) followed by $4 \%$ PFA (5 ml, for 3 minutes). Left and right carotid arteries were excised, further fixed overnight and embedded in paraffin or in OCT. Ligated vessels were sectioned from the aortic arch to the beginning of the ligature, and morphometric analysis was performed along the whole vessel length (approximately 9 mm). H\&E-stained paraffin-embedded sections were quantified for neointima or external area (external elastic layer area) using MetaMorph software. OCT sections were stained for Ki67 (ab16667, Abcam) proliferation marker at 1:100 dilution, followed by a fluorescent anti-Rabbit secondary antibody.

\section{Proteomic analysis based on isobaric tags for relative and absolute quantitation (iTRAQ).} Samples $(200 \mu \mathrm{g})$ were trypsin-digested separately using the whole proteome in-gel digestion protocol. ${ }^{18}$ Peptide iTRAQ labeling was performed essentially according to the manufacturer's instructions. After labeling, the samples were vacuum-dried and redissolved in $1 \%$ trifluoroacetic acid for both desalting and removal of excess labeling reagent in reversed-phase C-18 extraction cartridges (Oasis, Waters Corporation, Milford, MA, USA). High-resolution analysis of iTRAQlabeled peptides was carried out on an Easy nLC 1000 nano-HPLC apparatus (Thermo Scientific, San Jose, CA, USA) coupled to a linear ion trap Orbitrap mass spectrometer (Orbitrap Elite, 
Thermo Scientific). Peptides were suspended in $0.1 \%$ formic acid and then loaded onto a homemade C-18 reversed-phase nano-column (100 $\mu \mathrm{m}$ I.D., $45 \mathrm{~cm})$ and separated in a continuous gradient consisting of 8-31\% B for $180 \mathrm{~min}$ and 31-90\% B for 2 min (B = 90\% acetonitrile, 0.1\% formic acid) at $300 \mathrm{~nL} / \mathrm{min}$. Peptides were ionized using a Picotip emitter nanospray needle (New Objective, Woburn, MA, USA). Each MS run consisted of enhanced FT-resolution spectra (120,000 resolution) in the 390-1,200 m/z range followed by data-dependent MS/MS spectra of the 20 most intense parent ions acquired during the chromatographic run. The AGC target value in the Orbitrap for the survey scan was set to $1,000,000$. Fragmentation in the linear ion trap was performed at 32\% normalized collision energy with a target value of 10,000 ions. The full target was set to 30,000, with 1 microscan and a 100 ms injection time, and the dynamic exclusion was set to $0.5 \mathrm{~min}$. For peptide identification the MS/MS spectra were searched with the Sequest algorithm implemented in Proteome Discoverer 1.3 (Thermo Scientific). The results were analyzed using the probability ratio method, ${ }^{19}$ and a false discovery rate (FDR) for peptide identification was calculated based on the search results against a decoy database using the refined method. ${ }^{20}$ The iTRAQ reporter ion intensities were retrieved from MS/MS scans by QuiXoT software, ${ }^{21,22}$ and used as inputs to the weighted spectrum, peptide and protein statistical (WSPP) model, ${ }^{20}$ to obtain peptide and protein abundance changes. In a further step, proteins were classified into ontological categories according to the GO database, and abundance changes at the protein function level were analyzed as described. ${ }^{20}$ Protein or functional abundance changes were considered statistically significant when they had an FDR below 5\%.

\section{Proteomic analysis based on stable isotope labeling by amino acids in cell culture (SILAC).}

SILAC assay was performed as previously described, ${ }^{15}$ in highly proliferative cells. Briefly, bonemarrow-derived macrophages from wild-type or $M m p 17^{--}$mice were cultured in p100 plates (10 plates per genotype; pool of 3 mice per genotype) by seeding $6.5 \times 10^{6}$ cells per plate in SILAC medium (Invitrogen) supplemented with penicillin/streptomycin, L-glutamine, Hepes and 10\% bovine fetal serum, and isotopic amino acids (heavy Lysine and Arginine in wild-type, and light in $M m p 17^{\prime-}$ ). Supernatants (SN) were collected and processed as per the manufacturer's instructions. 
In brief, the SN of both genotypes were mixed 1:1 and enriched for glycosylated proteins by incubation with lectin WGA beads. Proteins were then reduced and alkylated, resolved by SDSPAGE, and stained with colloidal Coomassie, and 20 bands were cut. Bands were processed at the Vall d'Hebron Research Institute (Barcelona, Spain), where proteins were digested with trypsin and the obtained peptides analyzed by mass spectrometry. The data obtained were analyzed with Protein Scape 2.1 and WARP-LC 1.2 software platform (Bruker, Billerica, USA).

In vitro digestion assay and $\mathbf{N}$-terminal sequencing. Human recombinant OPN (500 to $2 \mu \mathrm{g}$; hrOPN, R\&D, 1433-OP/CF) were incubated with $500 \mathrm{ng}$ of the catalytic domain of human recombinant MMP17 (hrMMP17, 475940, Calbiochem, Billerica, USA) in digestion buffer (50 $\mathrm{mM}$ Tris-HCl, $10 \mathrm{mM} \mathrm{CaCl}$, $80 \mathrm{mM} \mathrm{NaCl}$, [pH7.4]) for 2 hours at $37^{\circ} \mathrm{C}$. Samples were separated by $12 \%$ SDS-PAGE and transferred to nitrocellulose membranes (Bio-Rad $45 \mu \mathrm{m}$ ). Full-length OPN and fragments were detected with anti-C-terminal OPN antibody recognizing aminoacids 234 to 240 of the human OPN sequence $(1 \mathrm{H} 3 \mathrm{~F} 7) .{ }^{23}$ An anti-mouse secondary antibody was used. Nterminal sequencing was performed according to Alphalyse guidelines (www.alphalyse.com/nterm_preparation_guideline.html, Palo Alto, USA). Briefly, 6 g of hrOPN and $3 \mu \mathrm{g}$ of hrMMP17 catalytic domain were incubated as described and the digestion mixture separated on a 4-16\% Tricine gel (Mini-Protean ${ }^{\circledR}$ Tris-Tricine precast gel; Bio-Rad). Proteins were transferred to a PVDF membrane (Immobilon-PSQ, Millipore), and the obtained OPN C-terminal cleavage-derived band in the membrane was visualized by Coomassie staining and used for Nterminal sequencing.

Lentiviral vector generation and infection. Lentiviral vectors were generated using the mouse full-length Mmp17 vector (ID 5716458, Invitrogen) or the E248A mutant, which was created using the QuikChange Site-Directed mutagenesis Kit (Agilent Technologies, Santa Clara, USA) with the following primers: Fw 5'-GTGGCCGTCCATGCGTTTGGTCATGCC-3' and RV 5'GGCATGACCAAACGCATGGACGGCCAC-3’. Vectors were cloned into the SFFV-IRESGFP lentiviral backbone and sequenced to check proper sequence mutation. Lentiviruses 
expressing Mock-GFP, Mmp17-GFP or Mmp17 E248A-GFP were prepared and titered as previously described. ${ }^{24}$ Mouse full length Opn was obtained from Sino Biological Inc. (MG50116-M), and subcloned into the same lentivirus backbone. N-terminal Opn were obtained with the following primers: FW 5' GGATCCGCATGAGATTGGCAGTGA 3', RV 5' CTAAAGCTGGGGAACAG 3’. Analysis of lentivirus-driven protein production was first analyzed by infection in HEK293 cells. Lentiviruses were injected intravenously at approximately $1 \times 10^{8} \mathrm{pfu} / \mathrm{ml}$. P1-stage mice were injected with $\sim 10 \mu \mathrm{l}$ of virus solution into the jugular vein after ice anesthesia, using a Hamilton syringe inserted through the neck skin. Mice were sacrificed 1 week (P8) or 8 weeks after injection. For injection into adult mice (8 weeksold), $100 \mu \mathrm{l}$ of virus solution $\left(1 \times 10^{8} \mathrm{pfu} / \mathrm{ml}\right)$ was injected into the jugular vein after skin excision, and mice were sacrificed 8 weeks later. Aortas were collected and processed for protein extraction, RNA extraction, microscopy or electron microscopy analysis according to the protocols described above.

Statistical analysis. The statistical analysis performed in each case is explained in detail in the corresponding figure legend. Data were analyzed by two-tailed Student's $t$-test or by one- or twoway ANOVA followed by the indicated comparison test for data with Gaussian normal distribution. Non-normal distributions were analyzed by Wilcoxon test or by one sample t-test for fold-induction comparisons. The one-tailed Fisher's exact test was used to analyze the incidence of aneurysm. For comparison of different groups or conditions, one- or two-way ANOVA was used, followed by the indicated comparison test in each case (Bonferroni or Tukei multiple comparison tests). Statistical tests were conducted with Prism 5 software (GraphPad Software, Inc., La Jolla, USA). Data are presented as mean \pm s.e.m. and differences were considered statistically significant at $\mathrm{p}<0.05:{ }^{*} \mathrm{p}$ value $<0.05$, ${ }^{* *} \mathrm{p}$ value $<0.01$, and ${ }^{* * *} \mathrm{p}$ value $<0.001$. 


\section{Supplemental References}

1. Regalado ES, Guo DC, Villamizar C, Avidan N, Gilchrist D, McGillivray B, Clarke L, Bernier F, Santos-Cortez RL, Leal SM, Bertoli-Avella AM, Shendure J, Rieder MJ, Nickerson DA, Project NGES, Milewicz DM. Exome sequencing identifies smad3 mutations as a cause of familial thoracic aortic aneurysm and dissection with intracranial and other arterial aneurysms. Circ Res. 2011;109:680-686

2. Li H, Durbin R. Fast and accurate long-read alignment with burrows-wheeler transform. Bioinformatics. 2010;26:589-595

3. Li H, Handsaker B, Wysoker A, Fennell T, Ruan J, Homer N, Marth G, Abecasis G, Durbin R, Genome Project Data Processing S. The sequence alignment/map format and samtools. Bioinformatics. 2009;25:2078-2079

4. Roy A, Kucukural A, Zhang Y. I-tasser: A unified platform for automated protein structure and function prediction. Nat Protoc. 2010;5:725-738

5. Ghoorah AW, Devignes MD, Smail-Tabbone M, Ritchie DW. Protein docking using case-based reasoning. Proteins. 2013;81:2150-2158

6. Sohail A, Marco M, Zhao H, Shi Q, Merriman S, Mobashery S, Fridman R. Characterization of the dimerization interface of membrane type 4 (mt4)-matrix metalloproteinase. J Biol Chem. 2011;286:33178-33189

7. Comeau SR, Gatchell DW, Vajda S, Camacho CJ. Cluspro: A fully automated algorithm for protein-protein docking. Nucleic Acids Res. 2004;32:W96-99

8. $\quad$ Rikimaru A, Komori K, Sakamoto T, Ichise H, Yoshida N, Yana I, Seiki M. Establishment of an mt4-mmp-deficient mouse strain representing an efficient tracking system for mt4-mmp/mmp-17 expression in vivo using beta-galactosidase. Genes Cells. 2007;12:1091-1100

9. Schneider CA, Rasband WS, Eliceiri KW. Nih image to imagej: 25 years of image analysis. Nature methods. 2012;9:671-675

10. Esteban V, Mendez-Barbero N, Jimenez-Borreguero LJ, Roque M, Novensa L, GarciaRedondo AB, Salaices M, Vila L, Arbones ML, Campanero MR, Redondo JM. Regulator of calcineurin 1 mediates pathological vascular wall remodeling. J Exp Med. 2011;208:2125-2139

11. Kanematsu Y, Kanematsu M, Kurihara C, Tsou TL, Nuki Y, Liang EI, Makino H, Hashimoto T. Pharmacologically induced thoracic and abdominal aortic aneurysms in mice. Hypertension. 2010;55:1267-1274

12. Daugherty A, Manning MW, Cassis LA. Antagonism of at2 receptors augments angiotensin ii-induced abdominal aortic aneurysms and atherosclerosis. British journal of pharmacology. 2001;134:865-870

13. Boettger T, Beetz N, Kostin S, Schneider J, Kruger M, Hein L, Braun T. Acquisition of the contractile phenotype by murine arterial smooth muscle cells depends on the mir143/145 gene cluster. J Clin Invest. 2009;119:2634-2647

14. Fogelstrand P, Feral CC, Zargham R, Ginsberg MH. Dependence of proliferative vascular smooth muscle cells on cd98hc (4f2hc, slc3a2). J Exp Med. 2009;206:23972406

15. Koziol A, Gonzalo P, Mota A, Pollan A, Lorenzo C, Colome N, Montaner D, Dopazo J, Arribas J, Canals F, Arroyo AG. The protease mt1-mmp drives a combinatorial proteolytic program in activated endothelial cells. FASEB J. 2012;26:4481-4494

16. Briones AM, Rodriguez-Criado N, Hernanz R, Garcia-Redondo AB, Rodrigues-Diez RR, Alonso MJ, Egido J, Ruiz-Ortega M, Salaices M. Atorvastatin prevents angiotensin ii-induced vascular remodeling and oxidative stress. Hypertension. 2009;54:142-149

17. Kumar A, Lindner V. Remodeling with neointima formation in the mouse carotid artery after cessation of blood flow. Arterioscler Thromb Vasc Biol. 1997;17:2238-2244

18. Bonzon-Kulichenko E, Perez-Hernandez D, Nunez E, Martinez-Acedo P, Navarro P, Trevisan-Herraz M, Ramos Mdel C, Sierra S, Martinez-Martinez S, Ruiz-Meana M, Miro-Casas E, Garcia-Dorado D, Redondo JM, Burgos JS, Vazquez J. A robust method 
for quantitative high-throughput analysis of proteomes by 18o labeling. Mol Cell Proteomics.10:M110 003335

19. Martinez-Bartolome S, Navarro P, Martin-Maroto F, Lopez-Ferrer D, Ramos-Fernandez A, Villar M, Garcia-Ruiz JP, Vazquez J. Properties of average score distributions of sequest: The probability ratio method. Mol Cell Proteomics. 2008;7:1135-1145

20. Navarro P, Vazquez J. A refined method to calculate false discovery rates for peptide identification using decoy databases. J Proteome Res. 2009;8:1792-1796

21. Lopez-Ferrer D, Ramos-Fernandez A, Martinez-Bartolome S, Garcia-Ruiz P, Vazquez J. Quantitative proteomics using 16o/18o labeling and linear ion trap mass spectrometry. Proteomics. 2006;6 Suppl 1:S4-11

22. Ramos-Fernandez A, Lopez-Ferrer D, Vazquez J. Improved method for differential expression proteomics using trypsin-catalyzed 180 labeling with a correction for labeling efficiency. Mol Cell Proteomics. 2007;6:1274-1286

23. Kazanecki CC, Kowalski AJ, Ding T, Rittling SR, Denhardt DT. Characterization of anti-osteopontin monoclonal antibodies: Binding sensitivity to post-translational modifications. Journal of cellular biochemistry. 2007;102:925-935

24. Garaulet G, Alfranca A, Torrente M, Escolano A, Lopez-Fontal R, Hortelano S, Redondo JM, Rodriguez A. Il10 released by a new inflammation-regulated lentiviral system efficiently attenuates zymosan-induced arthritis. Molecular therapy : the journal of the American Society of Gene Therapy. 2013;21:119-130 


\section{Online Figures}

A

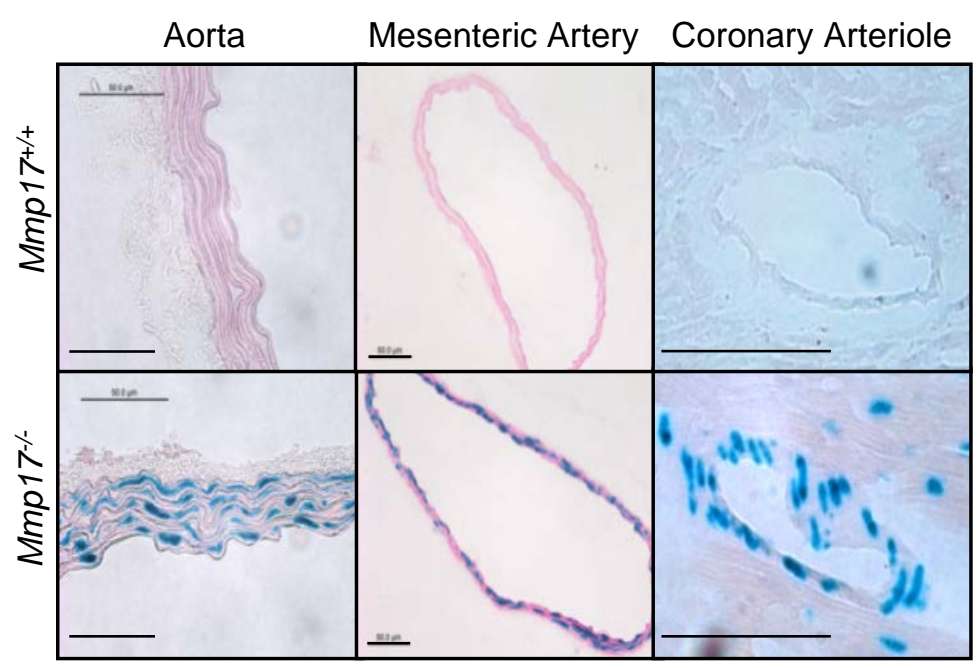

D

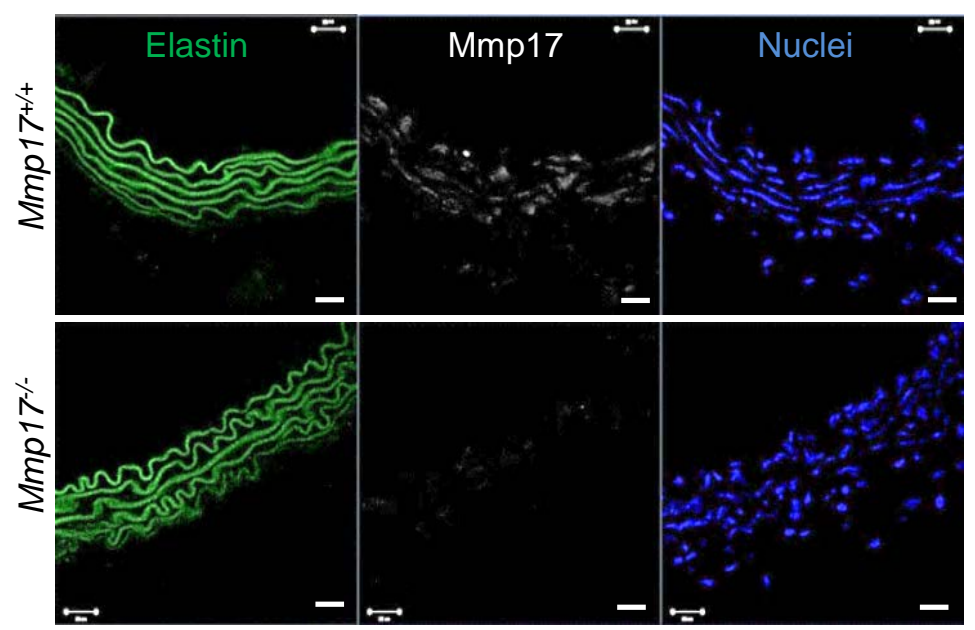

B

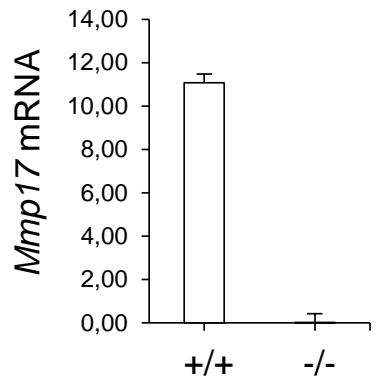

C

$\mathbf{E}$
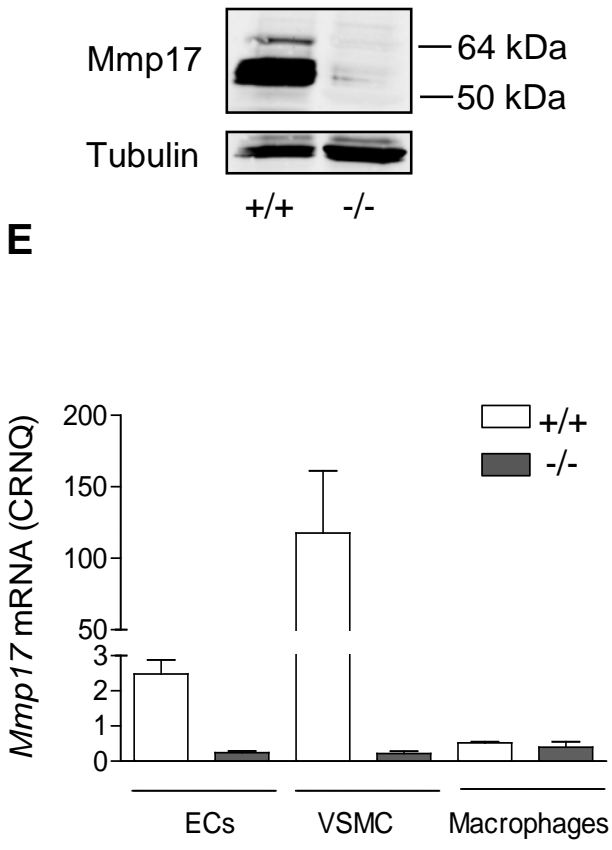

Online Figure I. Mmp17 is expressed in VSMCs of several arteries. A, Representative images of transverse sections of aortas, mesenteric arteries and coronary arterioles from wild-type and $M m p 17^{-}$ /- mice stained for LacZ ( $\mathrm{n}=$ 5-10 mice per genotype in 3 independent experiments). Scale bar 50 $\mu$. B-D, Mmp17 mRNA levels (Q-RT-PCR), protein expression (western-blot) and immunostaining in aortas from wild-type and $M m p 17^{-/-}$mice $(\mathrm{n}=30$ mice per genotype in 9 independent experiments in $\mathbf{B}$ and $\mathbf{C}$; $\mathrm{n}=5$ mice in D). Scale bar $20 \mu \mathrm{m}$. E, Mmp17 mRNA levels in VSMCs, endothelial cells (ECs) and macrophages from wild-type and Mmp17-null mice ( $\mathrm{n}=24$ mice per genotype in 3 independent experiments). Bars in $\mathbf{B}$ and $\mathbf{E}$ are means \pm s.e.m of calibrated relative normalized quantity (CRNQ) of RNA, obtained with qBase software. One representative experiment is shown in $\mathbf{B}$ and $\mathbf{E}$. 


\section{A}
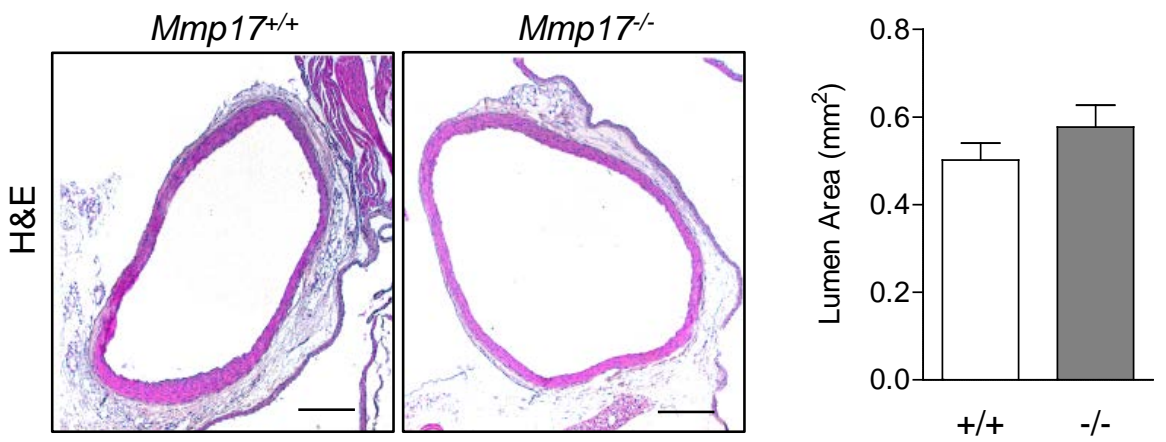

B

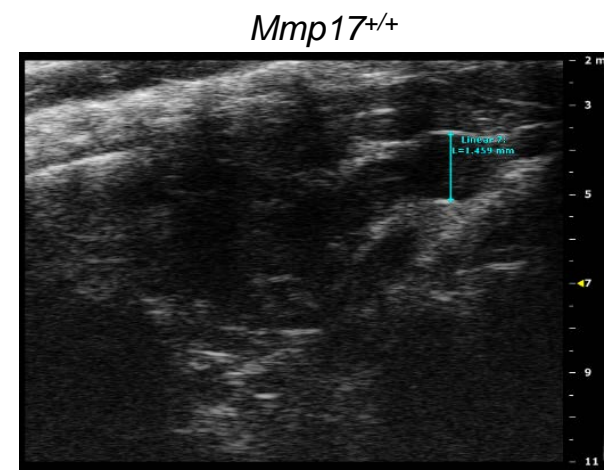

Mmp17\%-

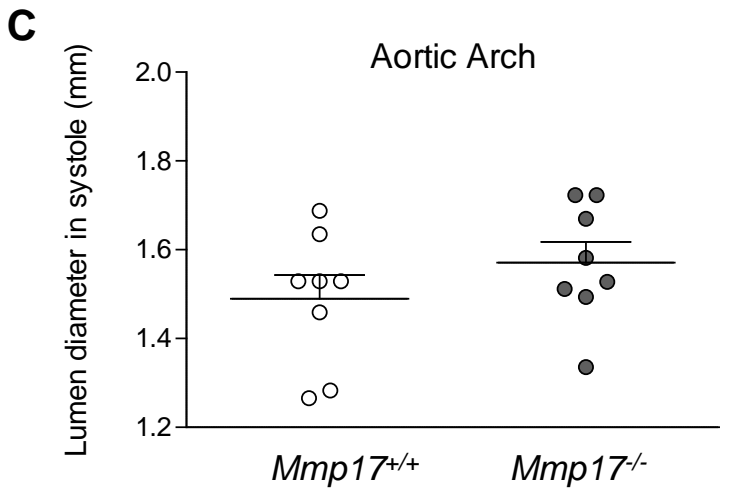

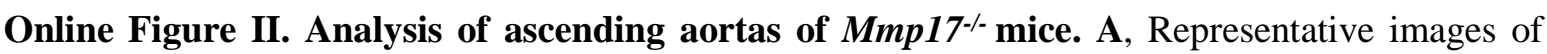
ascending aortas in transverse cut. The graph shows lumen area (right). $\mathrm{n}=5$ mice per genotype. Scale bar $200 \mu \mathrm{m}$. B, Echocardiography in adult mice showing larger aortic arch diameter in basal conditions in the Mmp17-null animal. C, Quantification of aortic arch diameter ( $\mathrm{n}=8$ mice per genotype). Data are means \pm s.e.m. and were tested by unpaired Student $t$-test in $\mathbf{A}(\mathrm{p}=0.3070)$ and C $(\mathrm{p}=0.1609)$. 

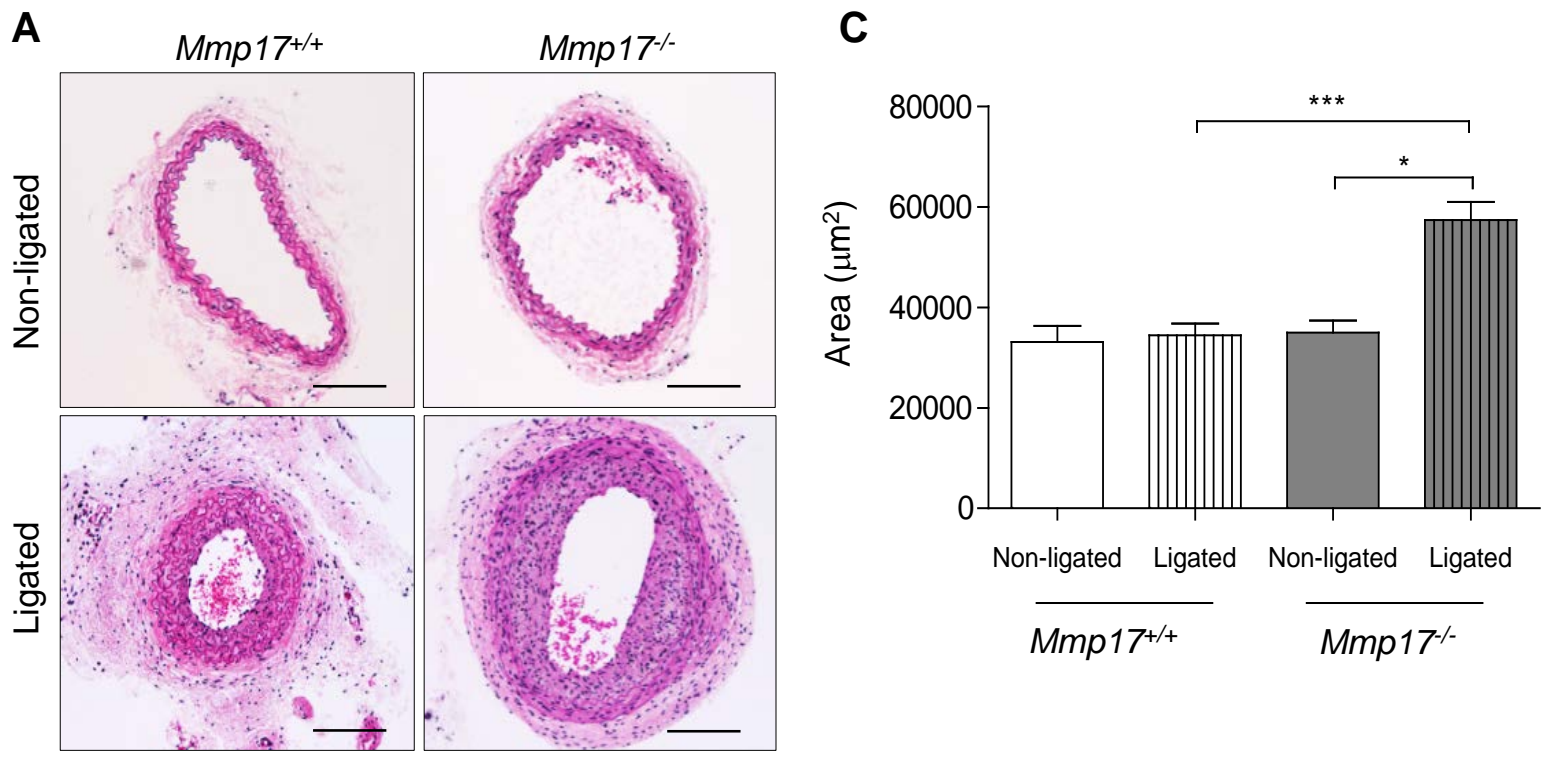

B

D
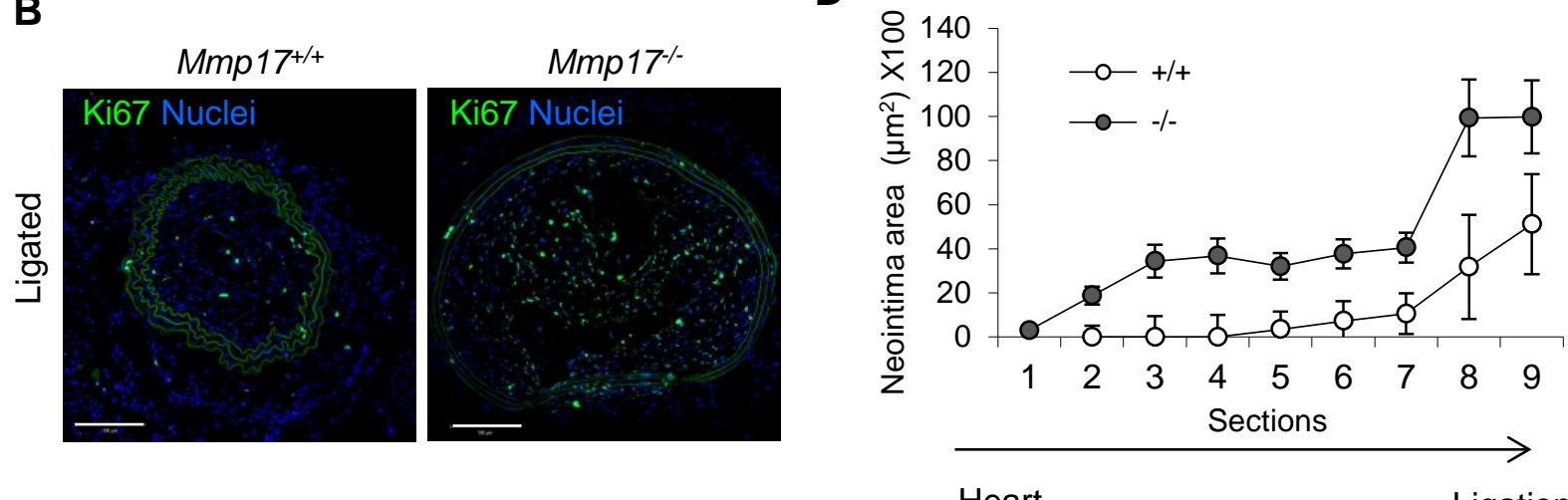

Heart

Ligation

Online Figure III. Carotid ligation induces outward remodeling and formation of a larger neointima in $\mathrm{Mmp17}^{-/}$mice. A, Representative images of sections stained with H\&E from right non-ligated (upper panel) and left ligated carotid arteries (lower panel) 28 days after ligation. Scale bar $100 \mu \mathrm{m}$. B, Representative confocal images of carotid arteries 28 days after ligation immunostained with anti-Ki67 (green). Elastin (auto-fluorescence, green) and nuclei (Hoechst, blue) are also shown. Scale bar $100 \mu \mathrm{m}$. C, External elastic lamina (EEL) area in wild-type and Mmp17 ${ }^{-/}$carotid arteries and $\mathbf{D}$, neointima area quantified along the carotid artery from the aortic arch to the ligation site. One representative experiment is shown of representative of 2 independent experiments. $\mathrm{n}=10$ mice per genotype. Numerical data in $\mathbf{C}$ and $\mathbf{D}$ are presented as means \pm s.e.m. and were tested by one-way ANOVA (*** $\mathrm{p}<0.001)$ followed by Tukey's multiple comparison, (* $\left.\mathrm{p}<0.05,{ }^{* * *} \mathrm{p}<0.001\right)$ in $\mathbf{C}$ and two-way ANOVA $(* * * \mathrm{p}<0.001)$ in $\mathbf{D}$. 
A
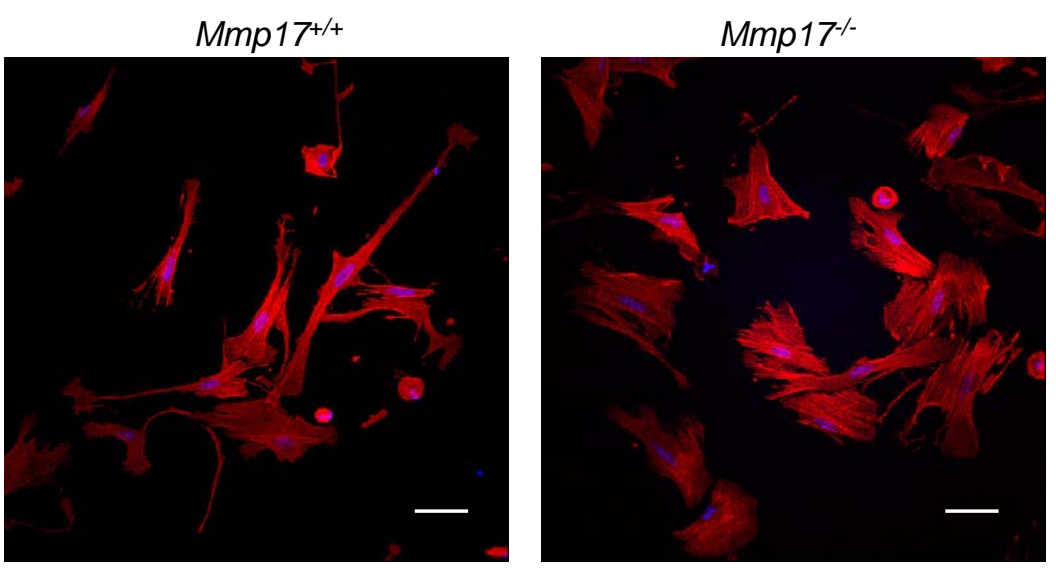

B

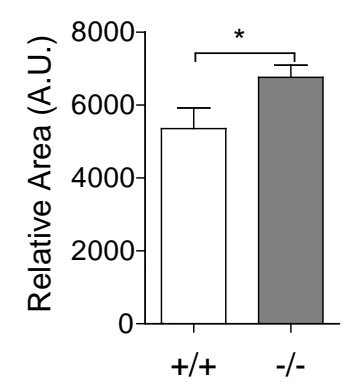

C

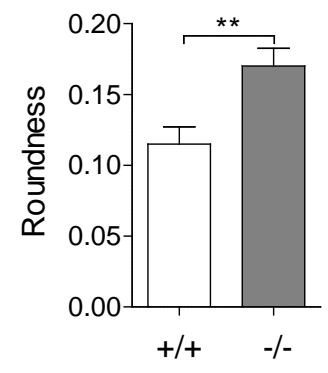

Online Figure IV. Mmp17 ${ }^{-/}$VSMCs display increased size and rounder morphology. A, Representative epifluorescence microscopy images from cultured VSMCs stained for F-actin (phalloidin, red). Scale bar $10 \mu \mathrm{m}$. B-C, Cellular area and roundness (cellular shape factor) quantified in cultured VSMCs isolated from wild-type and Mmp17-null aortas (A.U., arbitrary units). Data are presented as means of \pm s.e.m.; and were tested by unpaired Student's $t$-test $(*$ $\left.\mathrm{p}<0.5,{ }^{* *} \mathrm{p}<0.01\right)(\mathrm{n}=5$ independent experiments performed; 100 cells quantified per genotype). 
A

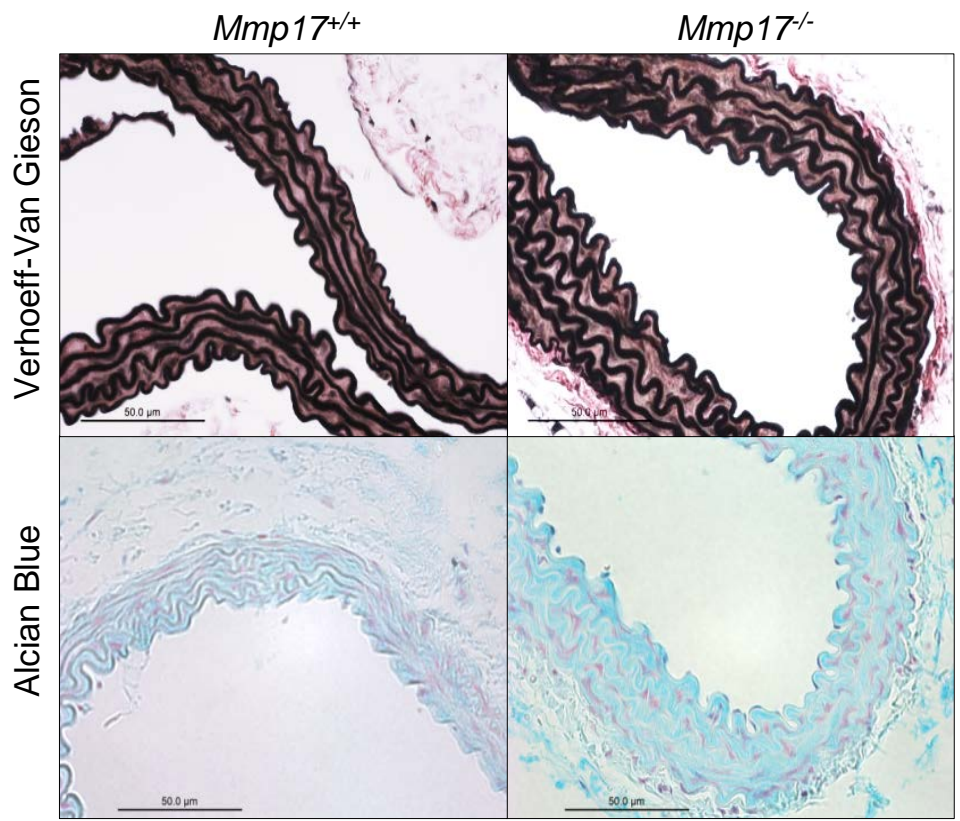

B
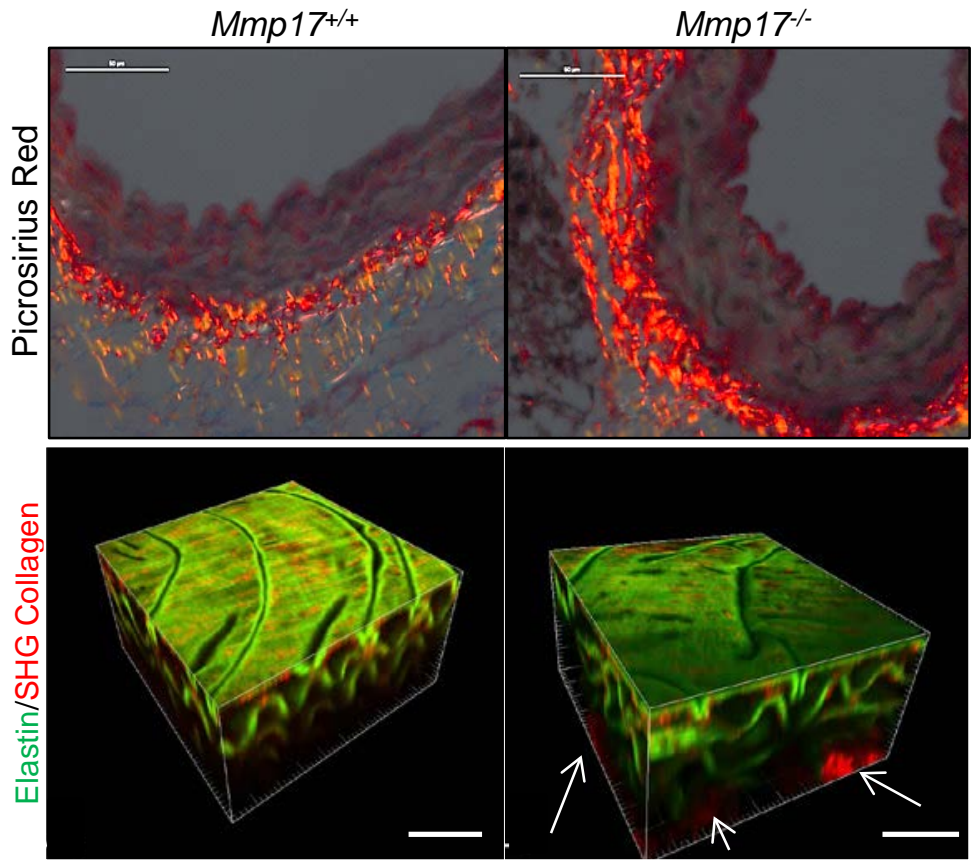

Online Figure V. ECM composition and distribution in Mmp17 ${ }^{-/}$aortas. A, Representative images of transverse sections of thoracic aortas from wild-type and Mmp17-/ mice stained for elastin (Verhoeff-van Gieson) and proteoglycans (Alcian blue) ( $\mathrm{n}=8$ to 12 mice per genotype analyzed in 2 independent experiments). Scale bar $50 \mu \mathrm{m}$. B, Representative images of transverse sections of thoracic aortas from wild-type and Mmp17/- mice stained for collagen (picrosirius red). Scale bar $50 \mu \mathrm{m}$. 3D-rendering reconstruction of second harmonic generation multi-photon microscopy images from wildtype and Mmp17- aortas (SHG fibrillar collagen in red, and auto-fluorescent elastin in green). Arrows point to collagen deposits in the adventitia of null mice ( $\mathrm{n}=4$ mice per genotype analyzed in 3 independent experiments). Scale bar $30 \mu \mathrm{m}$. 
A

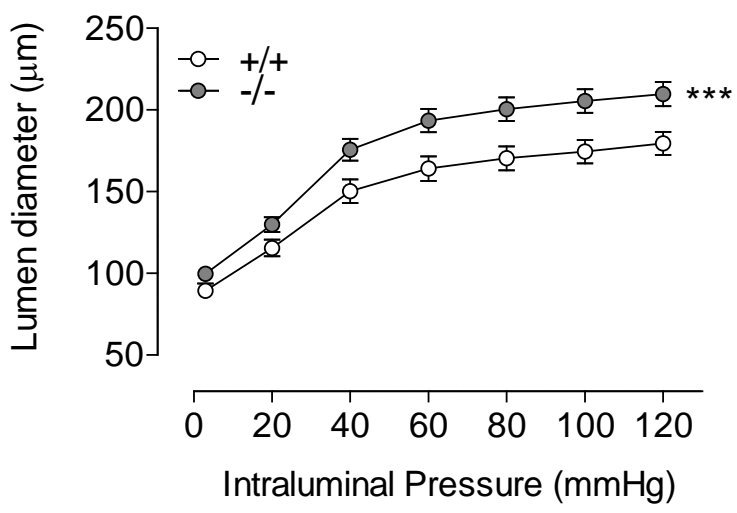

B

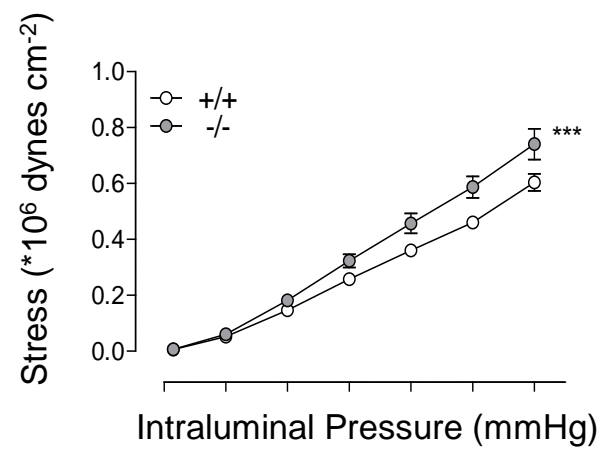

D

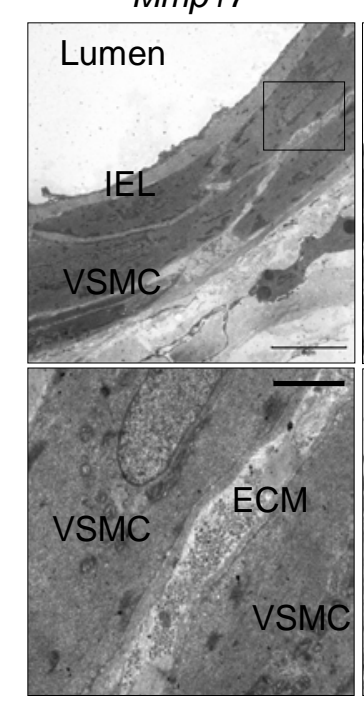

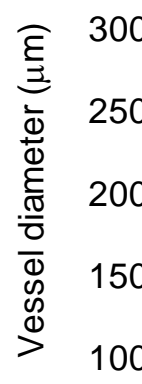

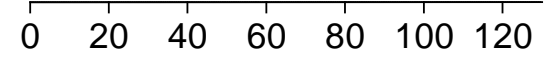

Intraluminal Pressure $(\mathrm{mmHg})$

C

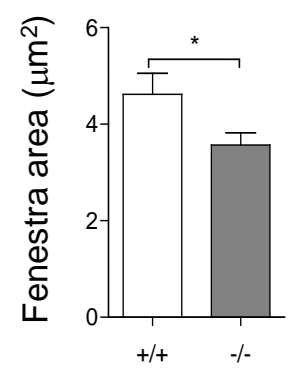

$\mathbf{E}$

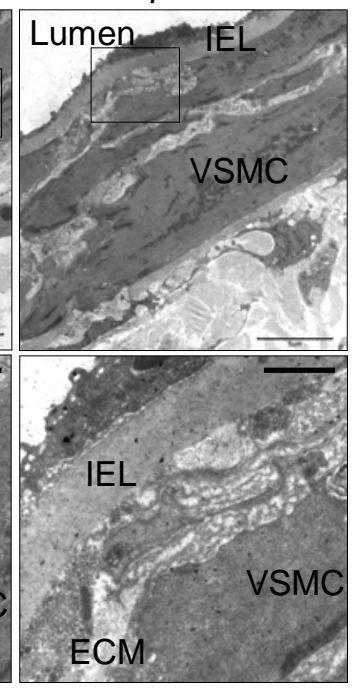

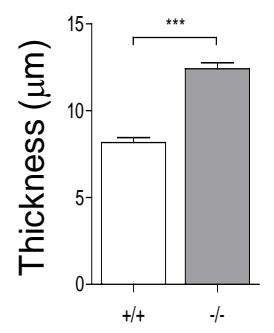

$\mathbf{F}$

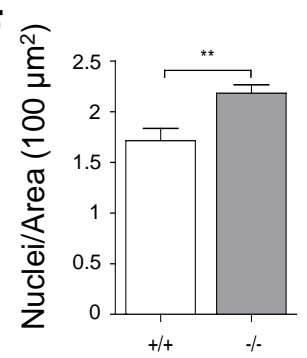

Online Figure VI. Mesenteric artery phenotype in Mmp17 ${ }^{-/-}$mice. A, Lumen and external diameter of mesenteric artery in wild-type and $M m p 17^{-/-}$mice in response to intraluminal pressures applied ex vivo between 0 and $120 \mathrm{mmHg}$ ( $\mathrm{n}=9$ mice per genotype). B, Vessel wall stress in response to intraluminal pressure in mesenteric arteries from wild-type and Mmp17-null mice and $\mathbf{C}$, fenestra area in the internal elastic lamina ( $\mathrm{n}=$ 9 mice per genotype analyzed in 3 independent experiments). D, Representative TEM images of mesenteric artery transverse sections from wild-type and $M m p 17^{-/-}$mice. IEL, internal elastic lamina. $\mathrm{n}=5$ mice per genotype. Scale bar $5 \mu \mathrm{m}$ and $0.5 \mu \mathrm{m}$ in inset (bottom). E-F, Vessel wall thickness and VSMC nuclear density in mesenteric arteries from wild-type or Mmp17-null mice. $\mathrm{n}=3$ mice per genotype (thickness) and 9 mice per genotype analyzed in 4 independent experiments (VSMC nuclear density). Data are means \pm s.e.m. and were tested by unpaired Student $t$-test in $\mathbf{C}, \mathbf{E}$ and $\mathbf{F}\left({ }^{*} \mathrm{p}<0.05\right.$, $\left.{ }^{* *} \mathrm{p}<0.01,{ }^{* * *} \mathrm{p}<0.001\right)$ and by two-way ANOVA $(* * * \mathrm{p}<0.001)$ in $\mathbf{A}$ and $\mathbf{B}$. 
A $M \operatorname{Mmp} 17^{+/ L a c Z}$

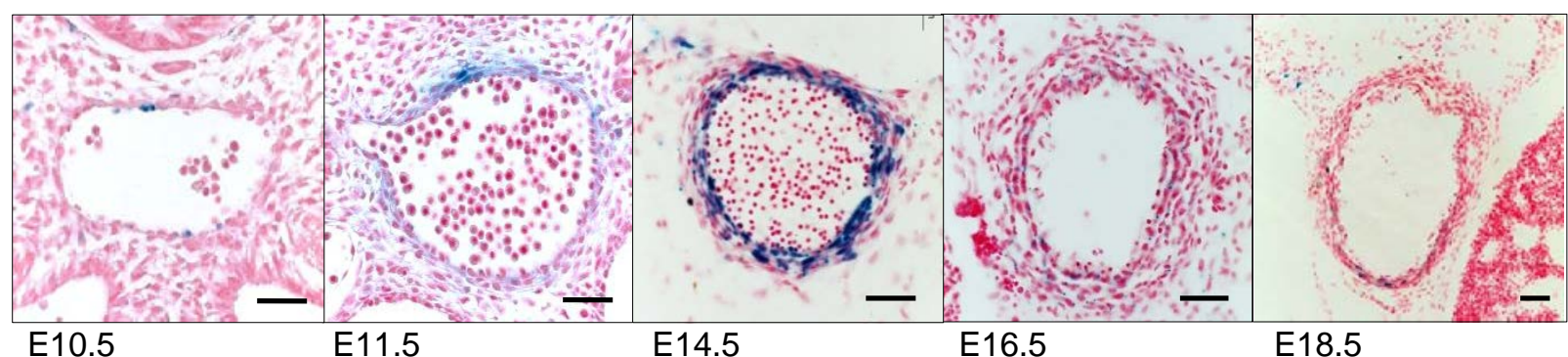

B
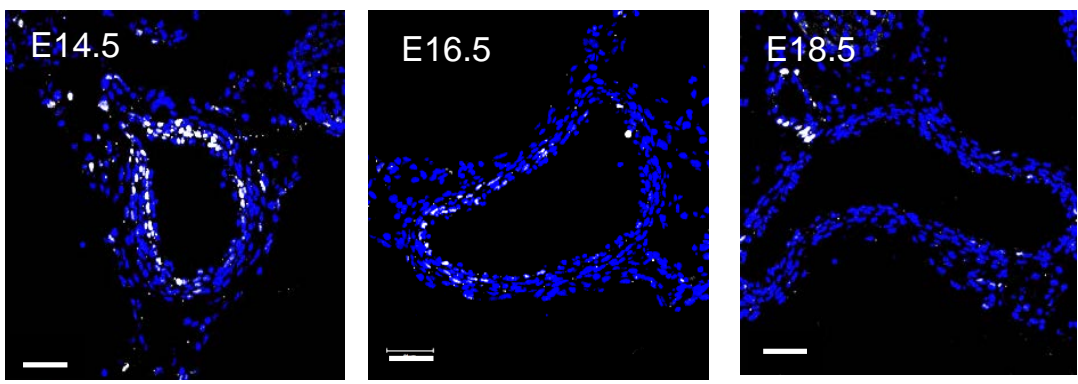

Online Figure VII. Mmp17 expression during embryonic aorta development. A, Representative images of LacZ staining in aortas from E10.5, E11.5, E14.5, E16.5 and E18.5 Mmp17+/LacZ embryos. $\mathrm{n}=4$ mice per time point. Scale bar $20 \mu \mathrm{m}$ (E10.5 and E11.5) and $50 \mu \mathrm{m}$ (E14.5 to 18.5). B, Representative confocal microscopy images of E14.5, E16.5 and E18.5 Mmp17 ${ }^{+/ L a c Z}$ embryo aortas showing anti- $\beta$-galactosidase nuclear immunostaining (white) and nuclei Hoechst (blue). Note abundant positive cells in the E14.5 medial layer compared to the few positive cells in E18.5 aorta. Scale bar $50 \mu \mathrm{m} . \mathrm{n}=2$ to 3 mice per genotype. 

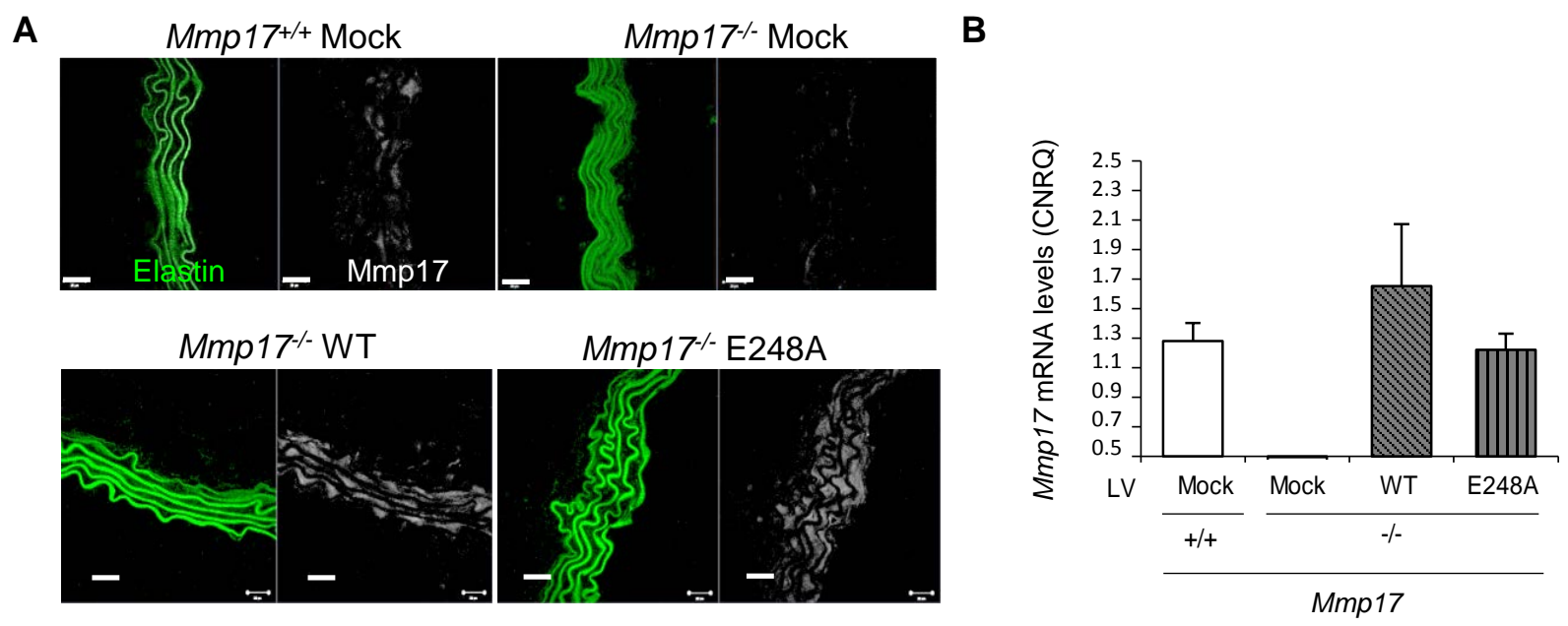

C
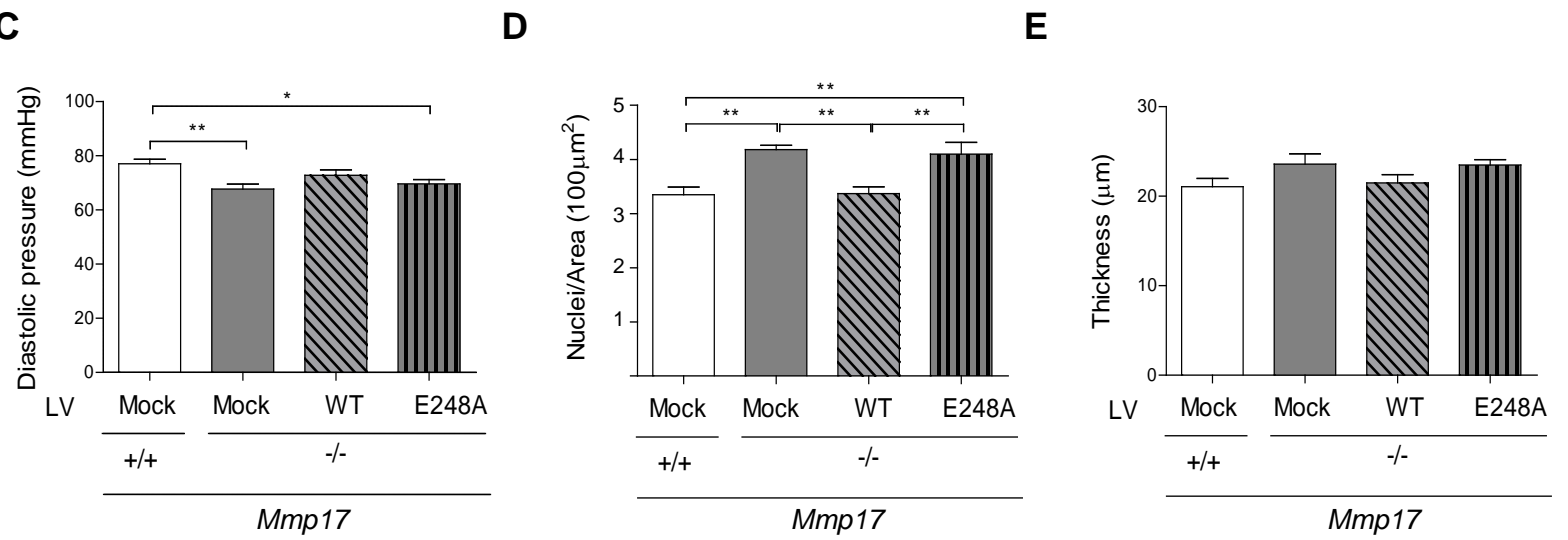

Online Figure VIII. Expression of catalytically active Mmp17 in Mmp17-null VSMCs of adult mice ameliorates the vascular phenotype. A, Representative immunofluorescence images of aortic sections stained for Mmp17 (white) 8 weeks after lentiviral injection of mock vector (SFFV-driven, IRES-GFP), Mmp17-WT or Mmp17-E248A vector at P1. $\mathrm{n}=19$ to 21 mice in 3 independent experiments. Scale bar $20 \mu \mathrm{m}$. Auto-fluorescent elastin, green. B, Mmp17 mRNA levels 8 weeks after injection at P1 of lentivirus encoding for Mmp17 ( $\mathrm{n}=12$ mice per genotype analyzed in 3 independent experiments). C-E, Diastolic pressure, VSMC nucleus density and media thickness, 8 weeks after lentiviral injection in adults (8-week-old). $n=9$ to 11 mice per condition in 3 independent experiments. Numerical data are means \pm s.e.m. of mRNA levels obtained in qBase analysis in B. Data in C-E were tested by one-way ANOVA $\left(\mathbf{C},{ }^{* *} \mathrm{p}<0.01, \mathbf{D},{ }^{* * *} \mathrm{p}<0.001\right)$ followed by Tukey's multiple comparison test $(* \mathrm{p}<0.05, * * \mathrm{p}<0.01)$. 


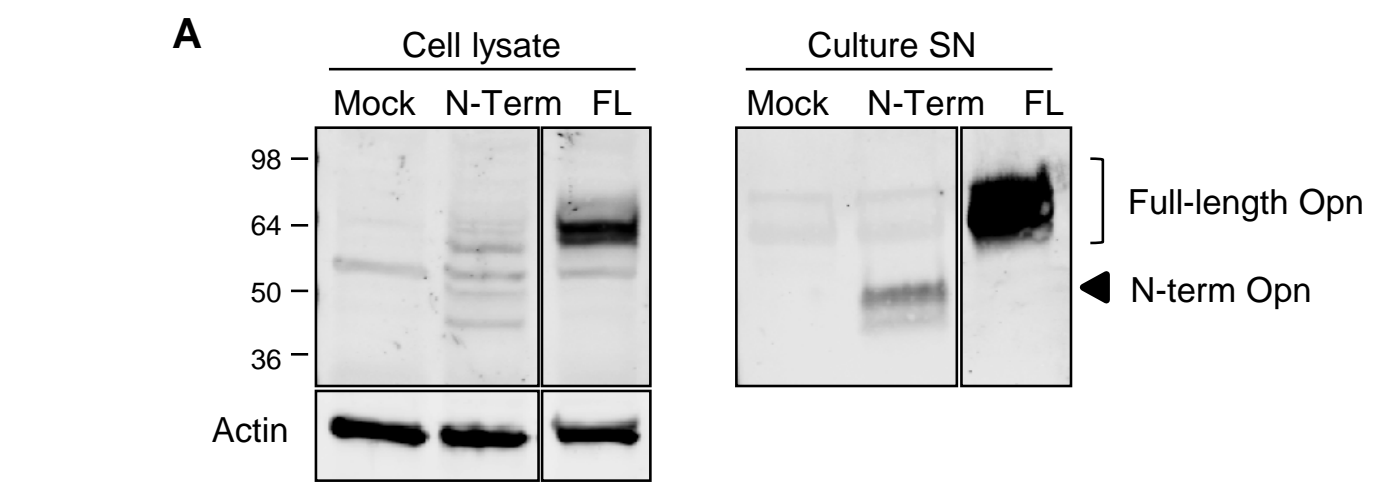

B
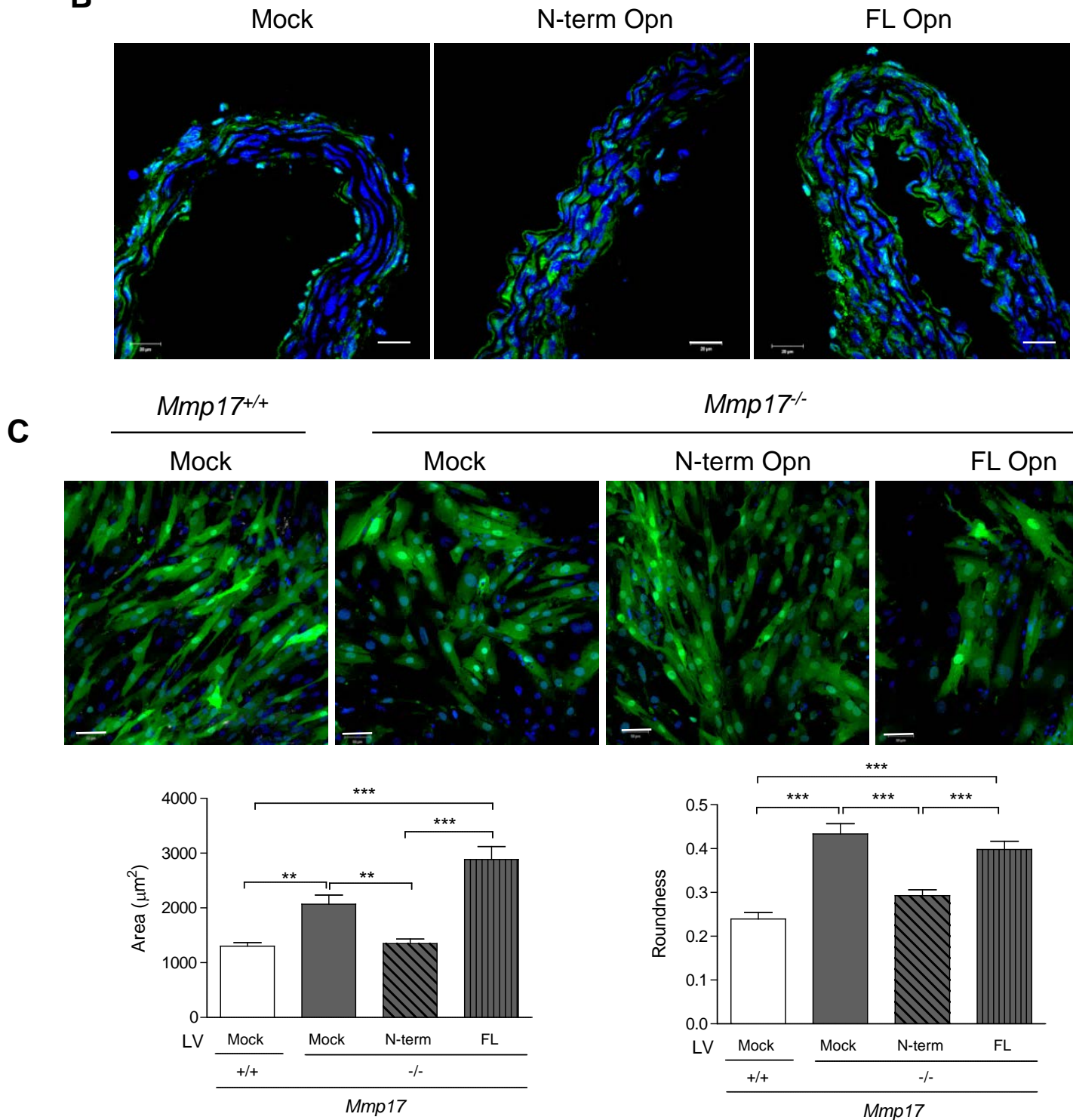

$\mathrm{Mmp} 17^{-/-}$
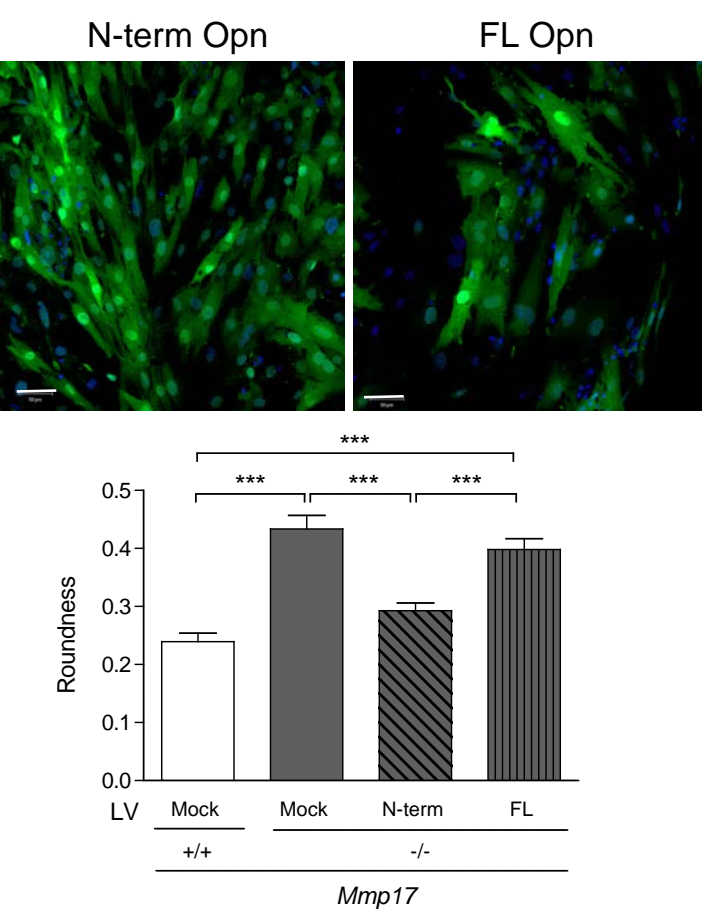

Online Figure IX. Expression of N-terminal Opn fragment in Mmp17-null VSMCs rescues VSMC morphological alterations. A, Western-blot of cell lysates and supernatants (SN) of HEK293 cells infected with mock, N-term or FL Opn lentivirus. B, Confocal images of anti-GFP immunostaining (green) of transverse aortic sections from P8 neonates 1 week after injection with mock, N-term or FL Opn lentivirus. Scale bar $20 \mu \mathrm{m}$. C, Representative pictures of wild-type and null VSMC infected with mock, Nterm or FL Opn lentivirus showing GFP-positive-infected cells. Scale bar $50 \mu \mathrm{m}$. Quantification of area and roundness are depicted. ( $\mathrm{n}=2$ independent experiments. One representative experiment is shown with 35 to 50 cells quantified per condition). Data are means \pm s.e.m. and were analyzed by one-way ANOVA $(* * * \mathrm{p}<0.001)$ followed by Bonferroni post-test $(* * \mathrm{p}<0.01, * * * \mathrm{p}<0.001)$. 
Online Movies I and II. Movies show echocardiography recording of the aortic arch of wild-type (Movie I) and Mmp17-null (Movie II) mice. 
Neonate (P7) and adult protein components of the GO categories found significantly altered in the iTRAQ proteomics experiment. Proteins were classified into GO categories and statistically significant differences at the protein function level were determined as explained in Supplemental Methods. Proteins are sorted according to Zq, the standardized log2-ratio of protein abundance, which describes the variation of protein log2-ratio around the grand mean of the experiment in units of standard deviation, according to the formulation of the WSPP model.

$>$ sp|Q9JI91|ACTN2_MOUSE Alpha-actinin-2 OS=Mus musculus GN=Actn2 PE=1 SV=2

$>$ sp|O89053|COR1A_MOUSE Coronin-1A OS=Mus musculus GN=Coro1a PE=1 SV=5

$>$ sp|Q8BTM8|FLNA_MOUSE Filamin-A OS=Mus musculus GN=Flna $P E=1 \mathrm{SV}=5$

tr|A1BN54|A1BN54_MOUSE Alpha actinin 1a OS=Mus musculus GN=Actn1 PE=2 SV=1

$>$ sp|Q01279|EGFR_MOUSE Epidermal growth factor receptor OS=Mus musculus GN=Egfr PE=1 SV=

$>$ sp|P26040|EZRI_MOUSE Ezrin OS=Mus musculus GN=Ezr PE $=1$ SV=3

$>$ sp|Q7TMB8|CYFP1_MOUSE Cytoplasmic FMR1-interacting protein 1 OS=Mus musculus GN=Cyfip

$>$ sp|P57780|ACTN4_MOUSE Alpha-actinin-4 OS=Mus musculus GN=Actn4 PE=1 SV=1

$>$ sp|Q61233|PLSL_MOUSE Plastin-2 OS=Mus musculus GN=Lcp1 PE=1 SV=4

$>$ sp|P28650|PURA1_MOUSE Adenylosuccinate synthetase isozyme 1 OS=Mus musculus GN=Adssl1 F

$>$ sp|Q9WUM4|COR1C_MOUSE Coronin-1C OS=Mus musculus GN=Coro1c PE=1 SV=2

$>$ sp|P26231|CTNA1_MOUSE Catenin alpha-1 OS=Mus musculus GN=Ctnna1 PE=1 SV=1

$>\mathrm{SP}$ Q9QYCO|ADDA_MOUSE Alpha-adducin OS=Mus musculus $\mathrm{GN}=\mathrm{Add} 1 \mathrm{PE}=1 \mathrm{SV}=2$

$>$ sp|Q6PB66|LPPRC_MOUSE Leucine-rich PPR motif-containing protein, mitochondrial OS=Mus mus

$>$ sp|Q7TPR4|ACTN1_MOUSE Alpha-actinin-1 OS=Mus musculus GN=Actn1 PE=1 SV=1

$>$ sp|A2ALU4|SHRM2_MOUSE Protein Shroom2 OS=Mus musculus GN=Shroom2 PE=1 SV=1

$>$ sp|Q9WUM3|COR1B_MOUSE Coronin-1B OS=Mus musculus GN=Coro1b PE=1 SV=1

sp|Q62418|DBNL_MOUSE Drebrin-like protein OS=Mus musculus GN=Dbnl PE=1 SV=2

$>$ sp|Q9QYB8|ADDB MOUSE Beta-adducin OS=Mus musculus GN=Add2 PE $=1 \mathrm{SV}=4$

$>$ sp|Q8K4L3|SVIL_MOUSE Supervillin OS=Mus musculus GN=Svil PE=1 SV=1

$>$ sp|Q6ZWR6|SYNE1_MOUSE Nesprin-1 OS=Mus musculus GN=Syne1 PE=1 SV=2

$>$ sp|Q61533|FSCN1_MOUSE Fascin OS=Mus musculus GN=Fscn1 PE=1 SV=4

sp|Q9ERG0|LIMA1_MOUSE LIM domain and actin-binding protein 1 OS=Mus musculus GN=Lima1 sp|P47757|CAPZB_MOUSE F-actin-capping protein subunit beta OS=Mus musculus GN=Capzb PE= sp|Q64331|MYO6_MOUSE Unconventional myosin-VI OS=Mus musculus GN=Myo6 PE=1 SV=1

$>$ sp|Q7TPW1|NEXN_MOUSE Nexilin OS=Mus musculus GN=Nexn PE $=1 \mathrm{SV}=3$

sp|Q6URW6|MYH14_MOUSE Myosin-14 OS=Mus musculus GN=Myh14 PE=1 SV=1

$>$ sp|P47753|CAZA1_MOUSE F-actin-capping protein subunit alpha-1 OS=Mus musculus GN=Capza1 1

$>$ sp|Q91XC0|AJUBA_MOUSE LIM domain-containing protein ajuba OS=Mus musculus GN=Ajuba PF

$>$ tr|Q3TN34|Q3TN34_MOUSE JRAB OS=Mus musculus GN=Micall2 PE=2 SV=1

sp|Q61792|LASP1_MOUSE LIM and SH3 domain protein 1 OS=Mus musculus GN=Lasp1 PE=1 SV= $\begin{aligned} & 0.137 \\ & 1.596\end{aligned}$

\begin{tabular}{|c|c|}
\hline $\mathrm{Zq}$ & rank/N \\
\hline-2.659 & 0.015 \\
-2.598 & 0.044 \\
-2.481 & 0.074 \\
-2.471 & 0.103 \\
-2.163 & 0.132 \\
-1.903 & 0.162 \\
-1.873 & 0.191 \\
-1.839 & 0.221 \\
-1.714 & 0.250 \\
-1.485 & 0.279 \\
-1.441 & 0.309 \\
-1.265 & 0.338 \\
-1.133 & 0.368 \\
-1.090 & 0.397 \\
-1.026 & 0.426 \\
-0.981 & 0.456 \\
-0.883 & 0.485 \\
-0.667 & 0.515 \\
-0.588 & 0.544 \\
-0.573 & 0.574 \\
-0.323 & 0.603 \\
-0.152 & 0.632 \\
-0.101 & 0.662 \\
-0.067 & 0.691 \\
-0.020 & 0.721 \\
0.000 & 0.750 \\
0.099 & 0.779 \\
0.103 & 0.809 \\
0.219 & 0.838 \\
0.273 & 0.868 \\
0.345 & 0.897 \\
0.459 & 0.926 \\
1.137 & 0.956 \\
1.596 & 0.985 \\
\hline
\end{tabular}

Sp|P28481|CO2A1_MOUSE Collagen alpha-1(II) chain OS=Mus musculus GN=Col2a1 PE

sp|Q3U962 $\mid$ CO5A2_MOUSE Collagen alpha-2(V) chain OS=Mus musculus GN=Col5a2 PE tr|B1AVK5|B1AVK5 MOUSE Procollagen type IV alpha 6 OS=Mus musculus GN=Col4a sp|P39061|COIA1_MOUSE Collagen alpha-1(XVIII) chain OS=Mus musculus GN=Col18a sp|P28301|LYOX_MOUSE Protein-lysine 6-oxidase OS=Mus musculus GN=Lox PE=1 SV sp|P11087|CO1A1_MOUSE Collagen alpha-1(I) chain OS=Mus musculus GN=Col1a1 PE=1 sp $\mid$ O88207|CO5A1_MOUSE Collagen alpha-1(V) chain OS=Mus musculus GN=Col5a1 PE $>$ sp|Q01149|CO1A2_MOUSE Collagen alpha-2(I) chain OS=Mus musculus GN=Col1a2 PE= sp|Q04857|CO6A1_MOUSE Collagen alpha-1(VI) chain OS=Mus musculus GN=Col6a1 PE sp|Q80X19|COEA1_MOUSE Collagen alpha-1(XIV) chain OS=Mus musculus GN=Col14a $>$ sp|Q8C6K9|CO6A6_MOUSE Collagen alpha-6(VI) chain OS=Mus musculus GN=Col6a6 P sp|A6H584|CO6A5_MOUSE Collagen alpha-5(VI) chain OS=Mus musculus GN=Col6a5 P sp|O35206|COFA1_MOUSE Collagen alpha- $1(\mathrm{XV})$ chain OS=Mus musculus GN=Col15a1 sp|P08122|CO4A2_MOUSE Collagen alpha-2(IV) chain OS=Mus musculus GN=Col4a2 PE $>$ sp|Q99K41|EMIL1_MOUSE EMILIN-1 OS=Mus musculus GN=Emilin1 PE=1 SV=1

sp|P02463|CO4A1_MOUSE Collagen alpha-1(IV) chain OS=Mus musculus GN=Col4a1

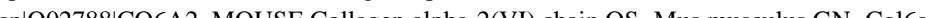
tr|B7ZNH7|B7ZNH7_MOUSE Collagen alpha-1(XIV) chain OS=Mus musculus GN=Col14 $>$ sp|Q60847|COCA1_MOUSE Collagen alpha-1(XII) chain OS=Mus musculus GN=Col12a1 $\mathrm{Zq}$ rank/N 


\begin{tabular}{|c|c|c|c|c|c|}
\hline Protein identification & $\mathrm{Zq}$ & rank/N & & -1.122 & \\
\hline$>$ tr|Q5FWB6|Q5FWB6_MOUSE MCG17387, isoform CRA_a OS=Mus musculus GN=Rplp0 PE=2 SV & -4.122 & 0.003 & >tr|Q8BWY8|Q8BWY8_MOUSE Putative uncharacterized protein (Fragment) OS=Mus musc & -0.997 & \\
\hline$>$ >sp|P62702|RS4X_MOUSE 40S ribosomal protein S4, $\mathrm{X}$ isoform OS=Mus musculus GN=Rps4x PE=2 & -3.903 & 0.009 & >sp|Q9WTI7|MYO1C_MOUSE Unconventional myosin-Ic OS=Mus musculus GN=Myo1c PI & -0.969 & \\
\hline >sp|Q9JJI8|RL38_MOUSE 60S ribosomal protein L38 OS=Mus musculus GN=Rpl38 PE=2 SV=3 & -3.675 & 0.016 & sp|O08638|MYH11_MOUSE Myosin-11 OS=Mus musculus GN=Myh11 PE=1 SV=1 & -0.965 & \\
\hline$>$ sp|Q6ZWV3|RL10_MOUSE 60S ribosomal protein L10 OS=Mus musculus GN=Rpl10 PE=2 SV=3 & -3.197 & 0.022 & >sp|Q6AW69|CGNL1_MOUSE Cingulin-like protein 1 OS=Mus musculus GN=Cgnl1 PE=1 ؛ & -0.084 & \\
\hline >sp|Q8BP67|RL24_MOUSE 60S ribosomal protein L24 OS=Mus musculus GN=Rpl24 PE=2 SV=2 & -2.545 & 0.028 & >sp|P59242|CING_MOUSE Cingulin OS=Mus musculus GN=Cgn PE=1 SV=1 & 0.379 & 81 \\
\hline$>$ tr|Q3TPD2|Q3TPD2_MOUSE Putative uncharacterized protein (Fragment) OS=Mus musculus GN=Iq: & -2.211 & 0.035 & >sp|Q9D0M5|DYL2_MOUSE Dynein light chain 2, cytoplasmic OS=Mus musculus GN=Dyn & 0.403 & \\
\hline >sp|P60122|RUVB1_MOUSE RuvB-like 1 OS=Mus musculus GN=Ruvbl1 PE=1 SV=1 & -2.159 & 0.041 & >sp|Q02566|MYH6_MOUSE Myosin-6 OS=Mus musculus GN=Myh6 PE=1 SV=2 & 3.701 & \\
\hline >sp|P62862|RS30_MOUSE 40S ribosomal protein S30 OS=Mus musculus GN=Fau PE=3 SV=1 & -2.137 & 0.047 & & & \\
\hline >sp|P62900|RL31_MOUSE 60S ribosomal protein L31 OS=Mus musculus GN=Rpl31 PE=2 SV=1 & -2.042 & 0.053 & Extracellular matrix GO:0031012 & & \\
\hline >sp|Q8CGC7|SYEP_MOUSE Bifunctional glutamate/proline--tRNA ligase OS=Mus musculus GN= & -2.025 & 0.060 & Protein identification & $\mathrm{Zq}$ & rank/N \\
\hline >sp|P62082|RS7_MOUSE 40S ribosomal protein S7 OS=Mus musculus GN=Rps7 PE=2 SV=1 & -1.846 & 0.066 & $>$ sp|Q61282|PGCA_MOUSE Aggrecan core protein OS=Mus musculus GN=Acan PE=1 $\mathrm{s}$ & -4.710 & \\
\hline >sp|O70133|DHX9_MOUSE ATP-dependent RNA he & -1.830 & 0.072 & >sp|P28481|CO2A1_MOUSE Collagen alpha-1(II) chain OS= & -3.672 & \\
\hline >sp|P62849|RS24_MOUSE 40S ribosomal protein S24 OS=Mus musculus GN=Rps24 PE=1 SV=1 & -1.829 & 0.079 & >sp|Q9QZZ6|DERM_MOUSE Dermatopontin OS=Mus musculus GN=Dpt PE=2 SV=1 & -3.617 & 0.031 \\
\hline$>$ tr|Q80UW7|Q80UW7_MOUSE IQ motif containing GTPase activating protein 1 OS=Mus musculus G & -1.810 & 0.085 & >sp|Q8BPB5|FBLN3_MOUSE EGF-containing fibulin-like extracellular matrix prote & -3.415 & 0.043 \\
\hline >sp|P62830|RL23_MOUSE 60S ribosomal protei & -1.682 & 0.091 & >sp|Q06890|CLUS_MOMOUSE Clusterin OS=Mus musc & -3.265 & \\
\hline >sp|Q9CQI7|RU2B_MOUSE U2 s & -1.656 & 0.097 & |>sp|P29788|V & -3.072 & \\
\hline >sp|Q6ZWY3|RS27L_MOUSE 40S ribosomal & -1.622 & 0.104 & >sp|Q91V88|NPNT_MOUSE Nephronectin OS=Mı & -3.021 & \\
\hline >sp|P97351|RS3A_MOUSE 40S ribosomal protein S3a OS=Mus musculus GN=Rps3a PE=1 SV=3 & -1.590 & 0.110 & >sp|Q3UTY6|THSD4_MOUSE Thrombospondin typ & -3.002 & \\
\hline >sp|Q9Z204|HNRPC_MOUSE Heterogeneous nuclear ribonuc & -1.588 & 0.116 & >sp|P39061|COIA1_MOUSE Collage & -2.832 & \\
\hline$>$ sp|P6 & -1.512 & 0.123 & >sp|P11276|FINC_MOUSE Fibronectin OS=Mus musculus GN= & -2.610 & \\
\hline >sp|P62281|RS11_MOUSE 40S ribosomal protein S11 OS=Mus musculus GN=Rps11 PE=2 SV=3 & -1.505 & 0.129 & Ius musculus GN=SfrF & -2.608 & \\
\hline >tr|Q8CDT3|Q8CDT3_MOUSE Pu & -1.465 & 0.135 & |>sp|O09164|SODE_MOL & -2.564 & \\
\hline MOUSE Exportin-1 OS & -1.461 & 0.142 & sp|Q8K4G1|LTBP4_MOUSE Latent-transformi & -2.490 & \\
\hline$>$ sp|Q3! & -1.457 & 0.148 & $>\operatorname{tr} \mid Q_{3} U_{H}^{3}$ & -2.457 & \\
\hline$>$ sp|P62855|RS26_MOUSE 40S ribosomal & -1.453 & 0.154 & >sp|Q99JR5|TINAL_MOUSE Tubulointerstitial nephritis antigen-lik & -2.434 & \\
\hline$>$ sp|P57780|ACTN4_MOUSE Al & -1.441 & 0.160 & >sp|P11087|CO1A1_M & -2.341 & \\
\hline$>$ sp|P27048|RSMB_MOUSE Sm & -1.434 & 0.167 & sp|P97873|LOXL1_MC & -2.209 & \\
\hline$>$ sp|Q61656|D & -1.424 & 0.173 & sp|Q9R118|HTRA1_MOUSE Serine pro & -2.193 & \\
\hline >sp|P57784|RU2A_MOUSE U2 small nuclear ribonucleop & -1.423 & 0.179 & Lactadherin OS=Mus musculus GN=N & -2.184 & \\
\hline$>\mathrm{sp} \mid \mathrm{Q} \mathrm{\theta}$ & -1.361 & 0.186 & sp $\mid 08820$ & -2.086 & \\
\hline$>$ sp|P62301|RS13_MOUSE 40S ribosomal & -1.341 & 0.192 & sp|P50608|FMC & -2.085 & \\
\hline$>$ sp|P32067|LA_MOUSE Lupus La protein & -1.326 & 0.198 & sp|Q05793|PGBM_M & -2.073 & \\
\hline$>$ sp|P62751|RL23A_MOUSE 60S ribosomal protein L23a OS=Mus musculus GN=Rpl23a PE=1 SV=1 & -1.272 & 0.204 & >sp|Q01339|APOH_MOUSE Beta-2-glycoprotein 1 OS=Mus musculus GN=Apoh PE=1 SV= & -2.071 & 0.278 \\
\hline$>$ sp|P09405| & -1.270 & 0.211 & & -2.071 & \\
\hline R4|RL17 MOUSE 60 & -1.257 & 0.217 & Lam & -1.923 & \\
\hline R9|RL34_MOUSE 60S r & -1.220 & 0.223 & sp|P21844|CM & -1.887 & \\
\hline$>$ sp|P14115|RL27A_MOUSE 60S ribosomal protein L27a OS=Mus musculus GN=Rpl27a PE=2 SV=5 & -1.139 & 0.230 & >tr|B1B0C7|B1B0C7_MOUSE Basement membrane-specific heparan sulfate proteoglycan cor & -1.887 & 0.327 \\
\hline >sp|P29341|PABP1_M & -1.106 & 0.236 & & -1.838 & \\
\hline$>\mathrm{sp} \mid \mathrm{Q} 6$ & -1.084 & 0.242 & & -1.782 & \\
\hline$>\mathrm{sp} \mid \mathrm{Q} 8$ & -1.067 & 0.248 & sp|Q8C6K9|CC & -1.617 & \\
\hline$>$ sp|Q921F4|HNRLL_MOUSE Heterogeneous nuclear ribonucleoprotein L-like OS=Mus muscul & -1.045 & 0.255 & sp|P28654|PGS2_MOUSE Decorin OS=Mus musculus GN=Dcn PE=2 SV=1 & -1.615 & \\
\hline & -1.036 & 0.261 & sp|Q640N1|AEBP1_MOUSE Adipocyte enhancer-binding protein 1 OS=Mus & -1.583 & \\
\hline$>$ sp|Q9CXW4|RL11_MOUSE 60S ribosc & -1.029 & 0.267 & sp|Q9JK & -1.559 & \\
\hline & -1.009 & 0.274 & 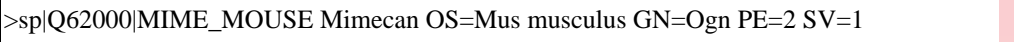 & -1.550 & \\
\hline$>$ sp|P19253|RL13A_MOUSE 60S ribosomal protein L13a OS=Mus musculus GN=Rpl13a PE=1 SV=4 & -0.910 & 0.280 & tr|A2AQ53|A2AQ53_MOUSE Fibrillin 1 OS=Mus musculus GN=Fbn1 PE=4 SV=1 & -1.539 & \\
\hline$>$ sp|P62717|RL18A_MOUSE 60S ribosomal protein L18a OS=Mus mus & -0.901 & 0.286 & sp|O35206|COFA1_MOUSE Collagen alpha-1(XV) chain OS=Mus musculus GN=c & -1.486 & \\
\hline & -0.898 & 0.292 & & 1.437 & \\
\hline & & & & & \\
\hline
\end{tabular}


sp|P47964|RL 36 MOUSE 60S ribosomal protein L36 OS=Mus musculus GN=Rpl36 PE=2 SV=2 sp|P12970|RL7A_MOUSE 60S ribosomal protein L7a OS=Mus musculus GN=Rpl7a PE=2 SV=2 $>$ sp|Q91VR5|DDX1_MOUSE ATP-dependent RNA helicase DDX1 OS=Mus musculus GN=Ddx1 PE $>$ sp|P62245|RS15A_MOUSE 40S ribosomal protein S15a OS=Mus musculus GN=Rps15a PE=2 SV=2 $>$ sp|Q9D0E1|HNRPM_MOUSE Heterogeneous nuclear ribonucleoprotein M OS=Mus musculus GN=H sp|P63017|HSP7C_MOUSE Heat shock cognate $71 \mathrm{kDa}$ protein OS=Mus musculus GN=Hspa8 PE=1 >sp|P62754|RS6_MOUSE 40S ribosomal protein S6 OS=Mus musculus GN=Rps6 PE=1 SV=1 sp|P62313|LSM6_MOUSE U6 snRNA-associated Sm-like protein LSm6 OS=Mus musculus GN=Lsm tr|Q3TT41|Q3TT41_MOUSE Putative uncharacterized protein OS=Mus musculus GN=Ncl PE=2 SV= sp|P62320|SMD3_MOUSE Small nuclear ribonucleoprotein Sm D3 OS=Mus musculus GN=Snrpd3 P sp|P47915|RL29_MOUSE 60S ribosomal protein L29 OS=Mus musculus GN=Rpl29 PE=2 SV=2 $>$ tr|Q91V55|Q91V55_MOUSE 40S ribosomal protein S5 OS=Mus musculus GN=Rps5 PE=2 SV=1 $>$ sp|P62274|RS29_MOUSE 40S ribosomal protein S29 OS=Mus musculus GN=Rps29 PE=2 SV=2 $>$ sp|Q6NVF9|CPSF6_MOUSE Cleavage and polyadenylation specificity factor subunit 6 OS=Mus musc $>$ sp|P97461|RS5_MOUSE 40S ribosomal protein S5 OS=Mus musculus GN=Rps5 PE=2 SV=3 tr|Q3TF92|Q3TF92_MOUSE Putative uncharacterized protein (Fragment) OS=Mus musculus GN=Dh: $>$ sp|P47962|RL5_MOUSE 60S ribosomal protein L5 OS=Mus musculus GN=Rpl5 PE=1 SV=3 $>$ sp|P62317|SMD2_MOUSE Small nuclear ribonucleoprotein Sm D2 OS=Mus musculus GN=Snrpd2 Pl $>$ sp|Q60668|HNRPD_MOUSE Heterogeneous nuclear ribonucleoprotein D0 OS=Mus musculus GN=H $>$ sp|Q921S7|RM37 MOUSE 39S ribosomal protein L37, mitochondrial OS=Mus musculus GN=Mrpl37 $>$ sp|P53026|RL10A_MOUSE 60S ribosomal protein L10a OS=Mus musculus GN=Rpl10a PE=1 SV=3 $>$ sp|P62305|RUXE_MOUSE Small nuclear ribonucleoprotein E OS=Mus musculus GN=Snrpe PE=2 ST sp|Q9CXY6|ILF2_MOUSE Interleukin enhancer-binding factor 2 OS=Mus musculus GN=Ilf2 PE=1 S $>$ sp|P14148|RL7_MOUSE 60S ribosomal protein L7 OS=Mus musculus GN=Rpl7 PE=2 SV=2 $>$ sp|P62264|RS14_MOUSE 40S ribosomal protein S14 OS=Mus musculus GN=Rps14 PE=2 SV=3 $>$ sp|P99027|RLA2_MOUSE 60S acidic ribosomal protein P2 OS=Mus musculus GN=Rplp2 PE=1 SV= $>$ sp|P58252|EF2_MOUSE Elongation factor 2 OS=Mus musculus GN=Eef2 PE $=1 \mathrm{SV}=2$

sp|P62918|RL8_MOUSE 60S ribosomal protein L8 OS=Mus musculus GN=Rpl8 PE=2 SV=2

sp|Q8R081|HNRPL_MOUSE Heterogeneous nuclear ribonucleoprotein L OS=Mus musculus GN=H $>$ sp|P62852|RS25_MOUSE 40S ribosomal protein S25 OS=Mus musculus GN=Rps25 PE=2 SV=1 $>$ sp|P62960|YBOX1_MOUSE Nuclease-sensitive element-binding protein 1 OS=Mus musculus GN=Yt $>$ sp|Q8VEK3|HNRPU_MOUSE Heterogeneous nuclear ribonucleoprotein U OS=Mus musculus GN=H tr|A2AW41|A2AW41_MOUSE Heterogeneous nuclear ribonucleoprotein R (Fragment) OS=Mus mus $>\operatorname{tr} \mid$ Q6P4T2|Q6P4T2 MOUSE Activating signal cointegrator 1 complex subunit 3-like 1 OS=Mus musc $>$ sp|Q61990|PCBP2_MOUSE Poly(rC)-binding protein 2 OS=Mus musculus GN=Pcbp2 PE=1 SV=1 $>$ sp|Q9EQK5|MVP_MOUSE Major vault protein OS=Mus musculus GN=Mvp PE $=1 \mathrm{SV}=4$ $>$ sp|Q9WTM5|RUVB2_MOUSE RuvB-like 2 OS=Mus musculus GN=Ruvbl2 PE=2 SV=3 $>$ sp|P35980|RL18_MOUSE 60S ribosomal protein L18 OS=Mus musculus GN=Rpl18 PE=2 SV=3 $>$ sp|Q6ZWN5|RS9_MOUSE 40S ribosomal protein S9 OS=Mus musculus GN=Rps9 PE=2 SV=3 $>$ sp|Q99020|ROAA_MOUSE Heterogeneous nuclear ribonucleoprotein A/B OS=Mus musculus GN= $>$ sp|P63276|RS17_MOUSE 40S ribosomal protein S17 OS=Mus musculus GN=Rps17 PE=1 SV=2 $>$ sp|O09167|RL21_MOUSE 60S ribosomal protein L21 OS=Mus musculus GN=Rpl21 PE=2 SV=3 $>$ sp|Q62376|RU17_MOUSE U1 small nuclear ribonucleoprotein $70 \mathrm{kDa}$ OS=Mus musculus GN=Snrnp tr|E9QNN1|E9QNN1_MOUSE ATP-dependent RNA helicase A OS=Mus musculus GN=Dhx9 PE=4 $>$ sp|P97499|TEP1_MOUSE Telomerase protein component 1 OS=Mus musculus GN=Tep1 PE=1 SV=1 >sp|Q9CR57|RL14_MOUSE 60S ribosomal protein L14 OS=Mus musculus GN=Rpl14 PE=2 SV=3 $>$ sp|P27659|RL3_MOUSE 60S ribosomal protein L3 OS=Mus musculus GN=Rpl3 PE=2 SV=3

$>$ sp|P62984|RL40 MOUSE Ubiquitin-60S ribosomal protein L40 OS=Mus musculus GN=Uba52 PE=

$>$ sp|Q99PV0|PRP8_MOUSE Pre-mRNA-processing-splicing factor 8 OS=Mus musculus GN=Prpf8 PE
$0.305 \mid>$ sp $\mid$ P08122|CO4A2_MOUSE Collagen alpha-2(IV) chain OS=Mus musculus GN=Co

$0.318>$ tr|J3QQ16|J3QQ16_MOUSE Protein Col6a3 OS=Mus musculus GN=Col6a3 PE=4 SV

. $324>$ sp|Q99K41|EMIL1_MOUSE EMILIN-1 OS=Mus musculus GN=Emilin1 $\mathrm{PE}=1 \mathrm{SV}=1$

.330 >tr|Q8BP04|Q8BP04_MOUSE Putative uncharacterized protein OS=Mus musculus GN=Loxl

6 >sp|O88322|NID2_MOUSE Nidogen-2 OS=Mus musculus GN=Nid2 PE=1 SV=2

$>\mathrm{sp|Q08879|FBLN1 \_ MOUSE} \mathrm{Fibulin-1} \mathrm{OS=Mus} \mathrm{musculus} \mathrm{GN=Fbln1} \mathrm{PE=1} \mathrm{SV=2}$

>sp|Q9DCT8|CRIP2_MOUSE Cysteine-rich protein 2 OS=Mus musculus GN=Crip2 PE=1

>tr|D3YWD1|D3YWD1_MOUSE Protein Col6a3 (Fragment) OS=Mus musculus GN=Col6a3 $>$ sp|Q8VEE1|LMCD1_MOUSE LIM and cysteine-rich domains protein 1 OS=Mus musculus $>$ sp|Q61292|LAMB2_MOUSE Laminin subunit beta-2 OS=Mus musculus GN=Lamb2 PE=2 >sp|Q02788|CO6A2_MOUSE Collagen alpha-2(VI) chain OS=Mus musculus GN=Col6a2 PE $>$ sp|Q80YX1|TENA_MOUSE Tenascin OS=Mus musculus GN=Tnc PE $=1 \mathrm{SV}=1$

$>$ sp|Q8CG19|LTBP1_MOUSE Latent-transforming growth factor beta-binding protein 1 OS= $>$ sp|Q9WVH9|FBLN5_MOUSE Fibulin-5 OS=Mus musculus GN=Fbln5 PE=2 SV=1 $>$ sp|P20352|TF_MOUSE Tissue factor OS=Mus musculus GN=F3 PE=1 SV=2

0.406 >tr|B7ZNH7|B7ZNH7_MOUSE Collagen alpha-1(XIV) chain OS=Mus musculus GN=Col14

>tr|Q59IW6|Q59IW6_MOUSE Protein Abi3bp OS=Mus musculus GN=Abi3bp PE=2 SV=1

0.418 >tr|B9EHT6|B9EHT6_MOUSE Fn1 protein OS=Mus musculus GN=Fn1 PE=2 SV=1

$0.425>\operatorname{tr|}|\mathrm{F} 8 \mathrm{VQJ} 3| \mathrm{F} 8 \mathrm{VQJ} 3 \_$MOUSE Laminin subunit gamma-1 OS=Mus musculus GN=Lamc1 PE=

0.431 >sp|P51885|LUM_MOUSE Lumican OS=Mus musculus GN=Lum PE=1 SV=2

$437>>$ sp|Q62219|TGFI1_MOUSE Transforming growth factor beta-1-induced transcript 1 protein $0.443>$ sp|P28653|PGS1_MOUSE Biglycan OS=Mus musculus GN=Bgn PE=2 SV=1

$0.450>$ sp|Q8CIZ8|VWF_MOUSE von Willebrand factor OS=Mus musculus GN=Vwf PE=1 SV=2

$456>$ >sp|P97298|PEDF_MOUSE Pigment epithelium-derived factor OS=Mus musculus GN=Serp

$.462>$ >p |O70503|DHB12_MOUSE Estradiol 17-beta-dehydrogenase $12 \mathrm{OS}=$ Mus musculus GN=H 0.469 > $>$ Q 0.475 >sp|Q9QUP5|HPLN1_MOUSE Hyaluronan and proteoglycan link protein $1 \mathrm{OS}=$ Mus muscul P07214|SPRC_MOUSE SPARC OS=Mus musculus GN=Sparc $\mathrm{PE}=1 \mathrm{SV}=1$ 
sp|P62270|RS18_MOUSE 40S ribosomal protein S18 OS=Mus musculus GN=Rps18 PE=1 SV=3 tr|E9Q3T0|E9Q3T0_MOUSE Uncharacterized protein OS=Mus musculus GN=Gm10073 PE=4 SV $>$ sp|P61358|RL27_MOUSE 60S ribosomal protein L27 OS=Mus musculus GN=Rp127 PE=2 SV=2 sp|Q91VM5|RMXL1_MOUSE RNA binding motif protein, X-linked-like-1 OS=Mus musculus GN=R $>$ sp |O35737|HNRH1_MOUSE Heterogeneous nuclear ribonucleoprotein $\mathrm{H}$ OS=Mus musculus GN=Hn sp|P62843|RS15 MOUSE 40S ribosomal protein S15 OS=Mus musculus GN=Rps15 PE=2 SV=2

sp|Q6ZWM4|NAA38_MOUSE N-alpha-acetyltransferase 38, NatC auxiliary subunit OS=Mus muscul $>$ sp|P86048|RL10L_MOUSE 60S ribosomal protein L10-like OS=Mus musculus GN=Rpl10l PE=2 SV= $>$ sp|Q9D823|RL37_MOUSE 60S ribosomal protein L37 OS=Mus musculus GN=Rpl37 PE=2 SV=3 tr|Q3U1C4|Q3U1C4_MOUSE MCG1271 OS=Mus musculus GN=Secisbp2 PE=2 SV=1

$>$ sp|P14131|RS16_MOUSE 40S ribosomal protein S16 OS=Mus musculus GN=Rps16 PE=2 SV=4 $>$ sp|Q9CZM2|RL15 MOUSE 60S ribosomal protein L15 OS=Mus musculus GN=Rpl15 PE=2 SV=4 SPIP2309|RUXG_MOUSE Small $>$ sp|P14206|RSSA_MOUSE 40S ribosomal protein SA OS=Mus musculus GN=Rpsa $P E=1$ SV=4 $>$ sp|P62311|LSM3_MOUSE U6 snRNA-associated Sm-like protein LSm3 OS=Mus musculus GN=Lsm $>$ sp|Q64012|RALY_MOUSE RNA-binding protein Raly OS=Mus musculus GN=Raly PE=1 SV=3 $>$ sp|P61255|RL26_MOUSE 60S ribosomal protein L26 OS=Mus musculus GN=Rpl26 PE=2 SV=1 $>$ sp|Q9CXW2|RT22_MOUSE 28S ribosomal protein S22, mitochondrial OS=Mus musculus GN=Mrps $>$ sp|Q9D0T1|NH2L1_MOUSE NHP2-like protein 1 OS=Mus musculus GN=Nhp2l1 PE=2 SV=4 $>$ sp|P62908|RS3_MOUSE 40S ribosomal protein S3 OS=Mus musculus GN=Rps3 PE=1 SV=1

$>$ sp|O35900|LSM2_MOUSE U6 snRNA-associated Sm-like protein LSm2 OS=Mus musculus GN=Lsm $>$ sp|Q9Z2X1|HNRPF_MOUSE Heterogeneous nuclear ribonucleoprotein F OS=Mus musculus GN=Hn $>$ sp|P35550|FBRL_MOUSE rRNA 2'-O-methyltransferase fibrillarin OS=Mus musculus GN=Fbl PE= Sp|P84099|RL19_MOUSE 60S ribosomal protein L19 OS=Mus musculus GN=Rpl19 PE=1 SV=1 sp|P14869|RLA0_MOUSE 60S acidic ribosomal protein P0 OS=Mus musculus GN=Rplp0 PE=1 SV=: $>$ sp|P62242|RS8_MOUSE 40S ribosomal protein S8 OS=Mus musculus GN=Rps8 PE=1 SV=2 $>$ sp|P47911|RL6_MOUSE 60S ribosomal protein L6 OS=Mus musculus GN=Rpl6 PE=1 SV=3 tr|Q6ZWZ6|Q6ZWZ6_MOUSE 40S ribosomal protein S12 OS=Mus musculus GN=Rps12 PE=2 SV $>$ sp|P67984|RL22_MOUSE 60S ribosomal protein L22 OS=Mus musculus GN=Rpl22 PE=2 SV=2 sp|Q9Z1X4|ILF3_MOUSE Interleukin enhancer-binding factor 3 OS=Mus musculus GN=Ilf3 PE=1 $>$ sp|P25444|RS2_MOUSE 40S ribosomal protein S2 OS=Mus musculus GN=Rps2 PE=1 SV=3 sp|Q5SSK3|TEFM_MOUSE Transcription elongation factor, mitochondrial OS=Mus musculus GN=T $>$ sp|P47955|RLA1_MOUSE 60S acidic ribosomal protein P1 OS=Mus musculus GN=Rplp1 PE=1 SV= $>$ sp|P49962|SRP09_MOUSE Signal recognition particle $9 \mathrm{kDa}$ protein OS=Mus musculus GN=Srp9 PE tr|Q8VHM5|Q8VHM5_MOUSE Heterogeneous nuclear ribonucleoprotein R OS=Mus musculus GN= $>$ sp|P63325|RS10_MOUSE 40S ribosomal protein S10 OS=Mus musculus GN=Rps10 PE=1 SV=1 $>$ sp|P35979|RL12_MOUSE 60S ribosomal protein L12 OS=Mus musculus GN=Rpl12 PE=1 SV=2 $>$ sp|P49312|ROA1_MOUSE Heterogeneous nuclear ribonucleoprotein A1 OS=Mus musculus GN=Hnr $>$ sp|Q9CX86|ROA0 MOUSE Heterogeneous nuclear ribonucleoprotein A0 OS=Mus musculus GN=Hn $>$ sp|Q9D8E6|RL4 MOUSE 60S ribosomal protein L4 OS=Mus musculus GN=Rpl4 PE=1 SV=3

$>$ sp|Q8BG05|ROA3_MOUSE Heterogeneous nuclear ribonucleoprotein A3 OS=Mus musculus GN=Hn $>$ sp $\mid$ O88569|ROA2_MOUSE Heterogeneous nuclear ribonucleoproteins A2/B1 OS=Mus musculus GN= $>$ sp|P60335|PCBP1_MOUSE Poly(rC)-binding protein $1 \mathrm{OS}=$ Mus musculus GN=Pcbp1 PE=1 SV=1 $>$ sp|P62889|RL30_MOUSE 60S ribosomal protein L30 OS=Mus musculus GN=Rpl30 PE=2 SV=2 sp|P70333|HNRH2_MOUSE Heterogeneous nuclear ribonucleoprotein H2 OS=Mus musculus GN=H sp|Q9JIK9|RT34_MOUSE 28S ribosomal protein S34, mitochondrial OS=Mus musculus GN=Mrps34 $>$ sp|P61979|HNRPK_MOUSE Heterogeneous nuclear ribonucleoprotein K OS=Mus musculus GN=Hn $>$ sp $\mid$ P60710|ACTB_MOUSE Actin, cytoplasmic 1 OS=Mus musculus GN=Actb PE $=1 \mathrm{SV}=1$

$>$ sp|P50580|PA2G4_MOUSE Proliferation-associated protein 2G4 OS=Mus musculus GN=Pa2g4 PE=1
$-0.0410 .613 \mid>$ sp $\mid$ Q5SX39|MYH4 MOUSE Myosin-4 OS=Mus musculus GN=Myh4 PE=1 SV=1

$-0.0290 .619>$ sp|Q03173|ENAH_MOUSE Protein enabled homolog OS=Mus musculus GN=Enah PE=1 $\begin{array}{ll}-0.024 & 0.626\end{array}>$ sp|Q91XD2|LIMS2_MOUSE LIM and senescent cell antigen-like-containing domain protei -0.022 0.632 >sp|P11688|ITA5_MOUSE Integrin alpha-5 OS=Mus musculus GN=Itga5 PE=1 SV=3

$-0.0090 .638>$ > 1 |Q8CGB6|TENC1_MOUSE Tensin-like C1 domain-containing phosphatase OS=Mus mus $-0.008 \quad 0.645>\mathrm{sp}|\mathrm{P} 26039| \mathrm{TLN} 1$ MOUSE Talin-1 OS=Mus musculus GN=Tln1 PE=1 SV=2

$0.0040 .651>$ sp|P09055|ITB1_MOUSE Integrin beta-1 OS=Mus musculus GN=Itgb1 PE=1 SV=1

$0.006 \quad 0.657>$ sp|Q7TPR4|ACTN1_MOUSE Alpha-actinin-1 OS=Mus musculus GN=Actn1 PE=1 SV=1

$0.056 \quad 0.664>$ sp|Q99JW4|LIMS1_MOUSE LIM and senescent cell antigen-like-containing domain protei $0.0560 .670>$ sp|Q9R1Z8|VINEX_MOUSE Vinexin OS=Mus musculus GN=Sorbs3 PE=1 SV=1

$0.0660 .676>$ sp $\mid$ P26041|MOES_MOUSE Moesin OS=Mus musculus GN=Msn PE=1 SV=3

$0.0720 .682>$ sp $\mid$ Q8VI36|PAXI_MOUSE Paxillin OS=Mus musculus GN=Pxn PE $=1 \mathrm{SV}=1$

$0.0740 .689>\operatorname{tr} \mid$ E9Q0S6|E9Q0S6_MOUSE Protein Tns1 OS=Mus musculus GN=Tns1 PE=4 SV=1

$0.0760 .695>>$ sp|P26040|EZRI_MOUSE Ezrin OS=Mus musculus GN=Ezr PE=1 SV=3

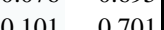

$\begin{array}{ll}0.101 & 0.701 \\ 0.126 & 0.708 \\ 0.153 & 0.714\end{array}$

$0.153 \quad 0.714$

0.174 0.720

$0.190 \quad 0.733$

$0.190 \quad 0.733$

作

作 sp|P26043|RADI_MOUSE Radixin OS=Mus musculus GN=Rdx PE=1 SV=3

sp|Q9EPC1|PARVA_MOUSE Alpha-parvin OS=Mus musculus GN=Parva PE=1 SV=1

Q61792|LASP1_MOUSE LIM and SH3 domain protein 1 OS=Mus musculus GN=Lasp1 |Q3UTJ2|SRBS2 MOUSE Sorbin and SH3 domain-containing protein $2 \mathrm{OS}=$ Mus muscul PBBFW7|LPP_MOUSE Lipoma-preferred partner homolog OS=Mus musculus GN=Lpp sp|Q62219|TGFI1_MOUSE Transforming growth factor beta-1-induced transcript 1 protein $>$ sp|Q71FD7|FBLI1_MOUSE Filamin-binding LIM protein 1 OS=Mus musculus GN=Fblim 作 sp|Q9JI91|ACTN2 MOUSE Alpha-actinin-2 OS=Mus musculus $\mathrm{GN}=\mathrm{Actn} 2 \mathrm{PE}=1 \mathrm{SV}=2$ D|O70309|ITB5_MOUSE Integrin beta-5 OS=Mus musculus GN=Itgb5 PE=1 SV=2 sp|Q64727|VINC_MOUSE Vinculin OS=Mus musculus GN=Vcl PE $=1 \mathrm{SV}=4$

sp|Q01768|NDKB_MOUSE Nucleoside diphosphate kinase B OS=Mus musculus GN=Nme tr|Q3U0Q9|Q3U0Q9_MOUSE Putative uncharacterized protein (Fragment) OS=Mus muscul sp|Q3TJD7|PDLI7_MOUSE PDZ and LIM domain protein 7 OS=Mus musculus GN=Pdlim 7 sp|Q62165|DAG1_MOUSE Dystroglycan OS=Mus musculus GN=Dag1 PE=1 SV=4 $>$ sp $|\mathrm{Q} 80 \mathrm{X} 90| \mathrm{FL} N \mathrm{~B}$ MOUSE Filamin-B OS=Mus musculus $\mathrm{GN}=\mathrm{Flnb} \mathrm{PE}=1 \mathrm{SV}=3$

sp|O55222|ILK_MOUSE Integrin-linked protein kinase OS=Mus musculus GN=Ilk PE=1 S sp|P34914|HYES_MOUSE Bifunctional epoxide hydrolase 2 OS=Mus musculus GN=Ephx2 $>$ sp|Q63844|MK03_MOUSE Mitogen-activated protein kinase 3 OS=Mus musculus GN=Ma sp|Q8CIB5|FERM2_MOUSE Fermitin family homolog 2 OS=Mus musculus GN=Fermt2 PE $>$ sp|Q9CVB6|ARPC2_MOUSE Actin-related protein 2/3 complex subunit 2 OS=Mus muscul $>\mathrm{sp}|\mathrm{P} 47226| T E S$ MOUSE Testin OS=Mus musculus GN $=$ Tes $P E=1 \mathrm{SV}=1$ sp|Q9ET54|PALLD_MOUSE Palladin OS=Mus musculus GN=Palld PE $=1 \mathrm{SV}=2$

sp|P31938|MP2K1_MOUSE Dual specificity mitogen-activated protein kinase kinase $1 \mathrm{OS}=$ sp|Q71LX4|TLN2_MOUSE Talin-2 OS=Mus musculus GN=Tln2 PE=1 SV=3 tr|Q3TN69|Q3TN69_MOUSE Putative uncharacterized protein (Fragment) OS=Mus muscul $0.890>$ sp|Q02566|MYH6_MOUSE Myosin-6 OS=Mus musculus GN=Myh6 PE=1 SV=2 
$>$ sp|O55142|RL35A_MOUSE 60S ribosomal protein L35a OS=Mus musculus GN=Rpl35a PE=2 SV=2 Sp|Q9ESX5|DKC1_MOUSE H/ACA ribonucleoprotein complex subunit 4 OS=Mus musculus GN=D >sp|P47963|RL13_MOUSE 60S ribosomal protein L13 OS=Mus musculus GN=Rpl13 PE=2 SV=3 $>$ sp|P83882|RL36A_MOUSE 60S ribosomal protein L36a OS=Mus musculus GN=Rpl36a PE=3 SV=2 $>$ sp|Q8CJG0|AGO2_MOUSE Protein argonaute-2 OS=Mus musculus GN=Eif2c2 PE=1 SV=3

$>$ sp|Q9CQR2|RS21_MOUSE 40S ribosomal protein S21 OS=Mus musculus GN=Rps21 PE=2 SV=1 $>$ sp|P62315|SMD1_MOUSE Small nuclear ribonucleoprotein Sm D1 OS=Mus musculus GN=Snrpd1 $>$ sp|P60867|RS20_MOUSE 40S ribosomal protein S20 OS=Mus musculus GN=Rps20 PE=1 SV=1 $>$ sp|Q8CJG1|AGO1_MOUSE Protein argonaute-1 OS=Mus musculus GN=Eif2c1 PE=1 SV=2 $>$ sp|P62858|RS28_MOUSE 40S ribosomal protein S28 OS=Mus musculus GN=Rps28 PE=2 SV=1 $>$ sp|P63323|RS12_MOUSE 40S ribosomal protein S12 OS=Mus musculus GN=Rps12 PE=1 SV=2 $>$ sp|P41105|RL 28_MOUSE 60S ribosomal protein L 28 OS=Mus musculus GN=Rpl28 PE=1 SV=2 $>$ sp|Q9CZX8|RS19 MOUSE 40S ribosomal protein $\mathrm{S} 19$ OS=Mus musculus GN=Rps19 PE=1 SV=3

\section{Mitochondrion GO:0005739}

sp|Q9CZT5|VASN_MOUSE Vasorin OS=Mus musculus GN=Vasn PE=2 SV=2

$>$ sp|Q9R257|HEBP1_MOUSE Heme-binding protein 1 OS=Mus musculus GN=Hebp1 PE=1 SV=2 $>$ sp|Q80U23|SNPH_MOUSE Syntaphilin OS=Mus musculus GN=Snph PE=1 SV=3

$>$ sp|P00329|ADH1_MOUSE Alcohol dehydrogenase $1 \mathrm{OS}=$ Mus musculus GN=Adh1 PE=2 SV=2

$>$ sp|Q924X2|CPT1B_MOUSE Carnitine O-palmitoyltransferase 1, muscle isoform OS=Mus musculus $>$ sp|Q8R2Y8|PTH2_MOUSE Peptidyl-tRNA hydrolase 2, mitochondrial OS=Mus musculus GN=Ptrh $>$ sp|O35459|ECH1_MOUSE Delta(3,5)-Delta(2,4)-dienoyl-CoA isomerase, mitochondrial OS=Mus mu sp|Q791T5|MTCH1_MOUSE Mitochondrial carrier homolog 1 OS=Mus musculus GN=Mtch1 PE=1 sp|O35658|C1QBP_MOUSE Complement component 1 Q subcomponent-binding protein, mitochondr $>$ sp|P98083|SHC1_MOUSE SHC-transforming protein 1 OS=Mus musculus GN=Shc1 PE=1 SV=3 $>$ sp|Q9CRD2|EMC2_MOUSE ER membrane protein complex subunit 2 OS=Mus musculus GN=Emc2 $>$ sp|P32020|NLTP_MOUSE Non-specific lipid-transfer protein OS=Mus musculus GN=Scp2 PE=1 SV sp|Q8CG76|ARK72_MOUSE Aflatoxin B1 aldehyde reductase member 2 OS=Mus musculus GN=Ak $>$ sp|Q8QZS1|HIBCH_MOUSE 3-hydroxyisobutyryl-CoA hydrolase, mitochondrial OS=Mus musculus sp|O09164|SODE_MOUSE Extracellular superoxide dismutase [Cu-Zn] OS=Mus musculus GN=Sod3 sp|Q9ERU9|RBP2_MOUSE E3 SUMO-protein ligase RanBP2 OS=Mus musculus GN=Ranbp2 PE=1 $>$ sp|Q9QX60|DGUOK_MOUSE Deoxyguanosine kinase, mitochondrial OS=Mus musculus GN=Dguok $>$ sp|P19157|GSTP1_MOUSE Glutathione S-transferase P 1 OS=Mus musculus GN=Gstp1 PE=1 SV=2 sp|P46638|RB11B_MOUSE Ras-related protein Rab-11B OS=Mus musculus GN=Rab11b PE=1 SV= $>$ sp|Q8BMP6|GCP60_MOUSE Golgi resident protein GCP60 OS=Mus musculus GN=Acbd3 PE=1 SV sp|O08756|HCD2_MOUSE 3-hydroxyacyl-CoA dehydrogenase type-2 OS=Mus musculus GN=Hsd17 sp|O55137|ACOT1_MOUSE Acyl-coenzyme A thioesterase 1 OS=Mus musculus GN=Acot1 PE=1 S $>$ sp|Q9Z1Q9|SYVC_MOUSE Valine--tRNA ligase OS=Mus musculus GN=Vars PE=2 SV=1

$>$ sp|P23927|CRYAB_MOUSE Alpha-crystallin B chain OS $=$ Mus musculus $G N=C$ ryab $P E=1 \mathrm{SV}=2$ $>$ sp|P59017|B2L13_MOUSE Bcl-2-like protein 13 OS=Mus musculus GN=Bcl2113 PE=1 SV=2

$>$ sp|Q8BWM0|PGES2_MOUSE Prostaglandin E synthase 2 OS=Mus musculus GN=Ptges2 PE $=1$ SV= $>$ sp|Q8BMD8|SCMC1_MOUSE Calcium-binding mitochondrial carrier protein SCaMC-1 OS=Mus mu sp $\mid$ P97807|FUMH_MOUSE Fumarate hydratase, mitochondrial OS=Mus musculus GN=Fh $P E=1 \mathrm{SV}=$ sp|P41216|ACSL1_MOUSE Long-chain-fatty-acid--CoA ligase 1 OS=Mus musculus GN=Acsl1 PE=1 sp|Q99LB6|MAT2B_MOUSE Methionine adenosyltransferase 2 subunit beta OS=Mus musculus GN= $>$ sp|P61222|ABCE1_MOUSE ATP-binding cassette sub-family E member 1 OS=Mus musculus GN=A sp|Q8R0Y6|AL1L1_MOUSE Cytosolic 10-formyltetrahydrofolate dehydrogenase OS=Mus musculus

>sp|Q9D0K2|SCOT1_MOUSE Succinyl-CoA:3-ketoacid coenzyme A transferase 1, mitochondrial OS=
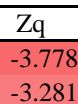
0.021
921 >sp|Q6URW6|MYH14_MOUSE Myosin-14 OS=Mus musculus GN=Myh14 PE=1 SV=1 sp|P11276|FINC_MOUSE Fibronectin OS=Mus musculus GN=Fn1 PE=1 SV=4 $0.934>$ tr|Q3UFT0|Q3UFT0_MOUSE Putative uncharacterized protein (Fragment) OS=Mus musc $0.940>$ >sp|Q8VDD5|MYH9_MOUSE Myosin-9 OS=Mus musculus GN=Myh9 PE=1 SV=4 0.947 >sp|Q3THE2|ML12B_MOUSE Myosin regulatory light chain 12B OS=Mus musculus GN=M (1) 959 >sp|Q61879|MYH10 MOUSE Myosin-10 OS=Mus musculus GN=Myh10 PE=1 SV=2 $>$ sp|Q07076|ANXA7_MOUSE Annexin A7 OS=Mus musculus GN=Anxa7 PE=2 SV=2 $>$ sp|Q8VI36|PAXI_MOUSE Paxillin OS=Mus musculus GN=Pxn PE=1 SV=1 sp|P26040|EZRI_MOUSE Ezrin OS=Mus musculus GN=Ezr PE=1 SV=3

sp|P19221|THRB_MOUSE Prothrombin OS=Mus musculus GN=F2 PE=1 SV=1 sp|Q9EPC1|PARVA_MOUSE Alpha-parvin OS=Mus musculus GN=Parva PE=1 SV=1 $>$ sp|Q71FD7|FBLI1_MOUSE Filamin-binding LIM protein $1 \mathrm{OS}=$ Mus musculus GN=Fblim 1 sp|Q9Z0P4|PALM_MOUSE Paralemmin-1 OS=Mus musculus GN=Palm PE=1 SV=1 sp|O08808|DIAP1_MOUSE Protein diaphanous homolog 1 OS=Mus musculus GN=Diaph1 sp|Q7TMB8|CYFP1_MOUSE Cytoplasmic FMR1-interacting protein 1 OS=Mus musculus sp|P27601|GNA13_MOUSE Guanine nucleotide-binding protein subunit alpha-13 OS=Mus sp|Q8BR92|PALM2_MOUSE Paralemmin-2 OS=Mus musculus GN=Palm2 PE=1 SV=1 $>$ sp|P39688|FYN_MOUSE Tyrosine-protein kinase Fyn OS=Mus musculus GN=Fyn PE=1 SI $>$ sp|Q8CIB5|FERM2_MOUSE Fermitin family homolog 2 OS=Mus musculus GN=Fermt2 PE $.009>$ >sp|Q8CIB5|FERM2_MOUSE Fermitin family homolog 2 OS=Mus musculus GN=Fermt2 PE $0.011>>$ sp $\mid$ O70318|E41L2_MOUSE Band 4.1-like protein 2 OS=Mus musculus GN=Epb4112 PE=1 0.014 >sp|O89053|COR1A_MOUSE Coronin-1A OS=Mus musculus GN=Coro1a PE=1 SV=5
$0.016>>$ sp|Q61738|ITA7_MOUSE Integrin alpha-7 OS=Mus musculus GN=Itga7 PE=1 SV=3 0.019 >sp|Q9JM96|BORG4_MOUSE Cdc42 effector protein 4 OS=Mus musculus GN=Cdc42ep4 PI 2.568

0.026

$0.028>s+Q$ $0.031>>033>$ |Q64331|MYO6_MOUSE Unconventional myosin-VI OS=Mus musculus GN=Myo6 PE= |Q69ZX3|Q69ZX3_MOUSE MKIAA0866 protein (Fragment) OS=Mus musculus GN=Myl Q5SX40|MYH1_MOUSE Myosin-1 OS=Mus musculus GN=Myh1 PE=1 SV=1 Q5SYD0|MYO1D_MOUSE Unconventional myosin-Id OS=Mus musculus GN=Myo1d |P13020|GELS_MOUSE Gelsolin OS=Mus musculus GN=Gsn PE=1 SV=3

Q9DAW9|CNN3_MOUSE Calponin-3 OS=Mus musculus GN=Cnn3 PE=2 SV=1 Pp|O70373|XIRP1_MOUSE Xin actin-binding repeat-containing protein 1 OS=Mus muscul Q8BVA4|LMOD1_MOUSE Leiomodin-1 OS=Mus musculus GN=Lmod1 PE=2 SV=1 Q8VDD5|MYH9_MOUSE Myosin-9 OS=Mus musculus GN=Myh9 PE=1 SV=4 Q08093|CNN2_MOUSE Calponin-2 OS=Mus musculus GN=Cnn2 PE=2 SV=1 Q6PDN3|MYLK_MOUSE Myosin light chain kinase, smooth muscle OS=Mus musculu P04919|B3AT_MOUSE Band 3 anion transport protein OS=Mus musculus GN=Slc4a1 |Q5SX39|MYH4_MOUSE Myosin-4 OS=Mus musculus GN=Myh4 PE=1 SV=1 p|Q03173|ENAH_MOUSE Protein enabled homolog OS=Mus musculus GN=Enah $P E=1$ |Q9R0Q6|ARC1A_MOUSE Actin-related protein 2/3 complex subunit 1A OS=Mus musc $\begin{aligned} & 0.078>\text { sp|P08032|SPTA1_MOUSE Spectrin alpha chain, erythrocytic } 1 \text { OS=Mus musculus GN=Spt. } \\ & 0.080\end{aligned}>$ sp|P26645|MARCS_MOUSE Myristoylated alanine-rich C-kinase substrate OS=Mus muscul 
$>$ sp|P05202|AATM_MOUSE Aspartate aminotransferase, mitochondrial OS=Mus musculus GN=Got 2 sp|P39688|FYN_MOUSE Tyrosine-protein kinase Fyn OS=Mus musculus GN=Fyn PE=1 SV=3 sp|P54869|HMCS2_MOUSE Hydroxymethylglutaryl-CoA synthase, mitochondrial OS=Mus musculus $>$ sp|Q9DCW4|ETFB_MOUSE Electron transfer flavoprotein subunit beta OS=Mus musculus GN=Etfb ] $>$ sp|Q9DCT2|NDUS3_MOUSE NADH dehydrogenase [ubiquinone] iron-sulfur protein 3, mitochondria $>$ sp|Q920E5|FPPS_MOUSE Farnesyl pyrophosphate synthase OS=Mus musculus GN=Fdps PE=2 SV= $>$ sp|Q64133|AOFA_MOUSE Amine oxidase [flavin-containing] A OS=Mus musculus GN=Maoa PE=1 $>$ sp|P56375|ACYP2_MOUSE Acylphosphatase-2 OS=Mus musculus GN=Acyp2 PE=2 SV=2 sp|P63328|PP2BA_MOUSE Serine/threonine-protein phosphatase 2B catalytic subunit alpha isoform C $>$ sp|P62897|CYC_MOUSE Cytochrome c, somatic OS=Mus musculus GN=Cycs PE=1 SV=2 $>$ sp|Q8BWT1|THIM_MOUSE 3-ketoacyl-CoA thiolase, mitochondrial OS=Mus musculus GN=Acaa2 $>$ sp|Q04447|KCRB_MOUSE Creatine kinase B-type OS=Mus musculus GN=Ckb PE=1 SV=1

sp|Q8BH95|ECHM_MOUSE Enoyl-CoA hydratase, mitochondrial OS=Mus musculus GN=Echs1 PE= $>$ sp|P97478|COQ7_MOUSE Ubiquinone biosynthesis protein COQ7 homolog OS=Mus musculus GN= $>$ sp|Q8K009|AL1L2_MOUSE Mitochondrial 10-formyltetrahydrofolate dehydrogenase OS=Mus muscu $>$ sp|P54071|IDHP_MOUSE Isocitrate dehydrogenase [NADP], mitochondrial OS=Mus musculus GN=I sp|Q62465|VAT1_MOUSE Synaptic vesicle membrane protein VAT-1 homolog OS=Mus musculus G $>$ sp|Q8CGK3|LONM_MOUSE Lon protease homolog, mitochondrial OS=Mus musculus GN=Lonp1 PI $>$ sp|Q8BMS1|ECHA_MOUSE Trifunctional enzyme subunit alpha, mitochondrial OS=Mus musculus $>$ sp|Q99JB2|STML2_MOUSE Stomatin-like protein 2 OS=Mus musculus GN=Stoml2 PE=1 SV=1

$>$ sp|Q9JM14|NT5C_MOUSE 5'(3')-deoxyribonucleotidase, cytosolic type OS=Mus musculus GN=Nt5c $>$ sp|Q78PY7|SND1_MOUSE Staphylococcal nuclease domain-containing protein 1 OS=Mus musculus sp|Q9D2G2|ODO2_MOUSE Dihydrolipoyllysine-residue succinyltransferase component of 2-oxoglut sp|P58281|OPA1_MOUSE Dynamin-like 120 kDa protein, mitochondrial OS=Mus musculus GN=Opa $>$ sp|Q99L13|3HIDH_MOUSE 3-hydroxyisobutyrate dehydrogenase, mitochondrial OS=Mus musculus ( $>$ sp|Q8BX02|KANK2_MOUSE KN motif and ankyrin repeat domain-containing protein 2 OS=Mus mu $>\operatorname{tr} \mid$ Q99K87|Q99K87_MOUSE Serine hydroxymethyltransferase OS=Mus musculus GN=Shmt2 PE=2 s $>\operatorname{tr}|\mathrm{Q} 3 \mathrm{U} 2 \mathrm{G} 2| \mathrm{Q} 3 \mathrm{U} 2 \mathrm{G} 2 \_$MOUSE Heat shock $70 \mathrm{kDa}$ protein $4 \mathrm{OS}=$ Mus musculus $\mathrm{GN}=\mathrm{Hspa} 4 \mathrm{PE}=2 \mathrm{SV}=$ $>$ sp|Q9D1G1|RAB1B_MOUSE Ras-related protein Rab-1B OS=Mus musculus GN=Rab1b PE=1 SV=1 $>$ sp|Q91ZP3-2|LPIN1_MOUSE Isoform 2 of Phosphatidate phosphatase LPIN1 OS=Mus musculus GN $>$ sp|P47738|ALDH2_MOUSE Aldehyde dehydrogenase, mitochondrial OS=Mus musculus GN=Aldh2 I >sp|Q6PB66|LPPRC_MOUSE Leucine-rich PPR motif-containing protein, mitochondrial OS=Mus mus $>$ sp|Q99JY0|ECHB_MOUSE Trifunctional enzyme subunit beta, mitochondrial OS=Mus musculus GN $>$ sp|P09671|SODM_MOUSE Superoxide dismutase [Mn], mitochondrial OS=Mus musculus GN=Sod2 $>$ sp|Q99MN9|PCCB_MOUSE Propionyl-CoA carboxylase beta chain, mitochondrial OS=Mus musculu $>$ sp|Q8BU85|MSRB3_MOUSE Methionine-R-sulfoxide reductase B3, mitochondrial OS=Mus musculu sp|Q8QZT1|THIL_MOUSE Acetyl-CoA acetyltransferase, mitochondrial OS=Mus musculus GN=Aca sp|Q05920|PYC_MOUSE Pyruvate carboxylase, mitochondrial OS=Mus musculus GN=Pc PE=1 SV= $>$ sp|Q9CZD3|SYG_MOUSE Glycine--tRNA ligase OS=Mus musculus GN=Gars PE=1 SV=1 $>$ sp|Q61191|HCFC1_MOUSE Host cell factor 1 OS=Mus musculus GN=Hcfc1 PE=1 SV=2 $>$ sp|Q07813|BAX_MOUSE Apoptosis regulator $\mathrm{BAX} O \mathrm{OS}=$ Mus musculus $\mathrm{GN}=\mathrm{Bax} \mathrm{PE}=1 \mathrm{SV}=1$

$>$ sp|Q3ULD5|MCCB_MOUSE Methylcrotonoyl-CoA carboxylase beta chain, mitochondrial OS=Mus n $>$ sp|Q9JHI5|IVD_MOUSE Isovaleryl-CoA dehydrogenase, mitochondrial OS=Mus musculus GN=Ivd P $>$ sp|P10639|THIO_MOUSE Thioredoxin OS=Mus musculus GN=Txn PE=1 SV=3

sp|Q63932|MP2K2_MOUSE Dual specificity mitogen-activated protein kinase kinase 2 OS=Mus mus sp|Q9JLN9|MTOR_MOUSE Serine/threonine-protein kinase mTOR OS=Mus musculus GN=Mtor PE $>$ sp|P50516|VATA_MOUSE V-type proton ATPase catalytic subunit A OS=Mus musculus GN=Atp6v1 $>$ sp|Q5U458|DJC11_MOUSE DnaJ homolog subfamily C member 11 OS=Mus musculus GN=Dnajc11

$>$ sp|Q9D880|TIM50_MOUSE Mitochondrial import inner membrane translocase subunit TIM50 OS=M
083 >sp|Q9JHJ0|TMOD3 MOUSE Tropomodulin-3 OS=Mus musculus GN=Tmod3 PE=1 SV=1 .085 >sp|Q62418|DBNL_MOUSE Drebrin-like protein OS=Mus musculus GN=Dbnl PE=1 SV=2 $0.088>\operatorname{tr} \mid \mathrm{D} 3 Y$ YV9|D3YVV9_MOUSE Synaptopodin-2 OS=Mus musculus GN=Synpo2 $\mathrm{PE}=4 \mathrm{~S}$ 0.090 >sp|P57780|ACTN4_MOUSE Alpha-actinin-4 OS=Mus musculus GN=Actn4 PE=1 SV=1 0.093 >sp|Q7TPW1|NEXN_MOUSE Nexilin OS=Mus musculus GN=Nexn PE=1 SV=3

0.095 >sp|P24452|CAPG_MOUSE Macrophage-capping protein OS=Mus musculus GN=Capg PE=1 $098>>$ sp|P21614|VTDB_MOUSE Vitamin D-binding protein OS=Mus musculus GN=Gc PE=1 SV $0.100>$ >sp|P40124|CAP1_MOUSE Adenylyl cyclase-associated protein $1 \mathrm{OS}=$ Mus musculus GN=C $0.102>$ sp|Q91YE8|SYNP2_MOUSE Synaptopodin-2 OS=Mus musculus GN=Synpo2 PE=1 SV=2 $0.105>$ sp|Q61879|MYH10_MOUSE Myosin-10 OS=Mus musculus GN=Myh10 PE=1 SV=2

$0.107>$ sp|Q9QXS6|DREB_MOUSE Drebrin OS=Mus musculus GN=Dbn1 PE=1 SV=4

$0.110>$ sp|P11531|DMD_MOUSE Dystrophin OS=Mus musculus GN=Dmd PE=1 SV=3

0.112 >sp|Q8VHX6|FLNC_MOUSE Filamin $-\mathrm{C} O \mathrm{OS}=$ Mus musculus $\mathrm{GN}=\mathrm{Fl} \mathrm{PE}=1 \mathrm{SV}=3$

0.115 >sp|Q3V0K9|PLSI_MOUSE Plastin-1 OS=Mus musculus GN=Pls1 PE=2 SV=1

0.117 >sp|P47754|CAZA2_MOUSE F-actin-capping protein subunit alpha-2 OS=Mus musculus GN $0.120>$ >sp|P26039|TLN1_MOUSE Talin-1 OS=Mus musculus GN=Tln1 PE=1 SV=2

0.122 >sp|P09055|ITB1_MOUSE Integrin beta-1 OS=Mus musculus GN=Itgb1 PE=1 SV=1

$>$ sp|P46735|MYO1B_MOUSE Unconventional myosin-Ib OS=Mus musculus GN=Myo1b PE $>$ tr|E9Q6R7|E9Q6R7_MOUSE Protein Utrn OS=Mus musculus GN=Utrn PE=4 SV=1 sp|Q8BTM8|FLNA_MOUSE Filamin-A OS=Mus musculus GN=Flna PE=1 SV=5 sp|Q91YR1|TWF1_MOUSE Twinfilin-1 OS=Mus musculus GN=Twf1 PE=1 SV=2 sp|Q7TPR4|ACTN1_MOUSE Alpha-actinin-1 OS=Mus musculus GN=Actn1 PE=1 SV=1 sp|P45591|COF2_MOUSE Cofilin-2 OS=Mus musculus GN=Cfl2 PE=1 SV=1 sp|P47753|CAZA1_MOUSE F-actin-capping protein subunit alpha-1 OS=Mus musculus GN $>$ sp|Q9CYT6|CAP2_MOUSE Adenylyl cyclase-associated protein 2 OS=Mus musculus GN= sp|Q08091|CNN1_MOUSE Calponin-1 OS=Mus musculus GN=Cnn1 PE=2 SV=1

$>\operatorname{tr} \mid$ Q99LB4|Q99LB4_MOUSE Capping protein (Actin filament), gelsolin-like OS=Mus mus tr|Q3TR59|Q3TR59_MOUSE Putative uncharacterized protein (Fragment) OS=Mus muscul $>$ sp|Q9WTI7|MYO1C_MOUSE Unconventional myosin-Ic OS=Mus musculus GN=Myo1c PI sp|O08638|MYH11_MOUSE Myosin-11 OS=Mus musculus GN=Myh11 PE=1 SV=1 $>$ sp|P26041|MOES_MOUSE Moesin OS=Mus musculus GN=Msn PE=1 SV=3 sp|Q99K51|PLST_MOUSE Plastin-3 OS=Mus musculus GN=Pls3 PE=1 SV=3 sp|Q8VD75|HIP1_MOUSE Huntingtin-interacting protein 1 OS=Mus musculus GN=Hip1 tr|E9Q0S6|E9Q0S6_MOUSE Protein Tns1 OS=Mus musculus GN=Tns1 PE=4 SV=1 sp|P58774|TPM2_MOUSE Tropomyosin beta chain OS=Mus musculus GN=Tpm2 PE=1 SV sp|P26040|EZRI_MOUSE Ezrin OS=Mus musculus GN=Ezr PE=1 SV=3

sp|P07091|S10A4_MOUSE Protein S100-A4 OS=Mus musculus GN=S100a4 PE=1 SV=1 sp|Q9JJ28|FLII_MOUSE Protein flightless-1 homolog OS=Mus musculus GN=Flii PE=1 S sp|P70460|VASP_MOUSE Vasodilator-stimulated phosphoprotein OS=Mus musculus GN= $>\mathrm{sp}|\mathrm{P} 26043| \mathrm{RADI}$ MOUSE Radixin OS=Mus musculus $\mathrm{GN}=\mathrm{Rdx} \mathrm{PE}=1 \mathrm{SV}=3$

0.181 >sp|Q9EPC1|PARVA_MOUSE Alpha-parvin OS=Mus musculus GN=Parva PE=1 SV=1

$184>>s p \mid$ Q61792|LASP1_MOUSE LIM and SH3 domain protein 1 OS=Mus musculus GN=Lasp1

$0.186>$ sp|Q99JY9|ARP3_MOUSE Actin-related protein 3 OS=Mus musculus GN=Actr3 PE=1 SV $0.189>$ sp|P15508|SPTB1_MOUSE Spectrin beta chain, erythrocytic OS=Mus musculus GN=Sptb P. $0.191>$ sp|P61161|ARP2_MOUSE Actin-related protein 2 OS=Mus musculus GN=Actr2 PE=1 SV= 0.194 >sp|Q61235|SNTB2_MOUSE Beta-2-syntrophin OS=Mus musculus GN=Sntb2 PE=1 SV=2 0.196 >sp|P47757-2|CAPZB_MOUSE Isoform 2 of F-actin-capping protein subunit beta $\mathrm{OS}=\mathrm{Mus}$ $0.199>$ sp|Q9JI91|ACTN2_MOUSE Alpha-actinin-2 OS=Mus musculus GN=Actn2 PE=1 SV=2

$0.201>$ sp|Q8K4L3|SVIL_MOUSE Supervillin OS=Mus musculus GN=Svil PE=1 SV=1
0.177
0.185
0.193
0.201
0.209
0.217
0.224
0.232
0.240
0.248
0.256
0.264
0.272
0.280
0.287
0.295
0.303
0.311
0.319
0.327
0.335
0.343
0.350
0.358
0.366
0.374
0.382
0.390
0.398
0.406
0.413
0.421
0.429
0.437
0.445
0.453
0.461
0.469
0.476
0.484
0.492
0.500
0.508
0.516
0.524
0.531
0.539
0.547
0.555 
$>$ sp|O55126|NIPS2_MOUSE Protein NipSnap homolog 2 OS=Mus musculus GN=Gbas PE=2 SV=1 $>$ sp|Q99MN1|SYK_MOUSE Lysine--tRNA ligase OS=Mus musculus GN=Kars $P E=1 \mathrm{SV}=1$ $>$ sp|Q99KI0|ACON_MOUSE Aconitate hydratase, mitochondrial OS=Mus musculus GN=Aco2 PE=1 S $>$ sp|Q8BP47|SYNC_MOUSE Asparagine--tRNA ligase, cytoplasmic OS=Mus musculus GN=Nars PE= $>$ sp|O54724|PTRF_MOUSE Polymerase I and transcript release factor OS=Mus musculus GN=Ptrf PE= $>$ sp|P38060|HMGCL_MOUSE Hydroxymethylglutaryl-CoA lyase, mitochondrial OS=Mus musculus G $>$ sp|P11499|HS90B_MOUSE Heat shock protein HSP 90-beta OS=Mus musculus GN=Hsp90ab1 PE=1 $>$ sp|Q9JMH6|TRXR1_MOUSE Thioredoxin reductase 1, cytoplasmic OS=Mus musculus GN=Txnrd1 $\mathrm{P}$ sp|Q9JHW2|NIT2_MOUSE Omega-amidase NIT2 OS=Mus musculus GN=Nit2 PE=1 SV=1 $>$ sp|P45952|ACADM_MOUSE Medium-chain specific acyl-CoA dehydrogenase, mitochondrial OS=M $>$ sp|Q8K1J6|TRNT1_MOUSE CCA tRNA nucleotidyltransferase 1, mitochondrial OS=Mus musculus C $>$ sp|Q9QXB9|DRG2_MOUSE Developmentally-regulated GTP-binding protein 2 OS=Mus musculus G. sp|Q8BYC6|TAOK3_MOUSE Serine/threonine-protein kinase TAO3 OS=Mus musculus GN=Taok3 $>$ sp|P07901|HS90A_MOUSE Heat shock protein HSP 90-alpha OS=Mus musculus GN=Hsp90aa1 PE= $>$ sp|Q9D0S9|HINT2_MOUSE Histidine triad nucleotide-binding protein 2, mitochondrial OS=Mus mus sp|Q6X893|CTL1_MOUSE Choline transporter-like protein 1 OS=Mus musculus GN=Slc44a1 PE=1 sp|P97742|CPT1A_MOUSE Carnitine O-palmitoyltransferase 1, liver isoform OS=Mus musculus GN= $>$ sp|Q91VR2|ATPG_MOUSE ATP synthase subunit gamma, mitochondrial OS=Mus musculus GN=AtF $>$ sp|Q8K4Z3|NNRE_MOUSE NAD(P)H-hydrate epimerase OS=Mus musculus GN=Apoa1bp PE=1 SV $>$ sp|P24270|CATA_MOUSE Catalase OS=Mus musculus GN=Cat PE $=1 \mathrm{SV}=4$

$>$ sp|Q9CWJ9|PUR9_MOUSE Bifunctional purine biosynthesis protein PURH OS=Mus musculus GN= $>$ sp|Q9Z2I0|LETM1_MOUSE LETM1 and EF-hand domain-containing protein 1, mitochondrial OS=M $>$ sp|Q922E6|FAKD2_MOUSE FAST kinase domain-containing protein 2 OS=Mus musculus GN=Fastk sp|Q922D8|C1TC_MOUSE C-1-tetrahydrofolate synthase, cytoplasmic OS=Mus musculus GN=Mthfo $>$ sp|P05132|KAPCA_MOUSE cAMP-dependent protein kinase catalytic subunit alpha OS=Mus muscul $>$ sp|Q921S7|RM37_MOUSE 39S ribosomal protein L37, mitochondrial OS=Mus musculus GN=Mrpl37 $>$ sp|P53026|RL10A_MOUSE 60S ribosomal protein L10a OS=Mus musculus GN=Rpl10a PE=1 SV=3 $>$ sp|O08553|DPYL2_MOUSE Dihydropyrimidinase-related protein 2 OS=Mus musculus GN=Dpysl2 P $>$ sp|P62264|RS14_MOUSE 40S ribosomal protein S14 OS=Mus musculus GN=Rps14 PE=2 SV=3 $>$ sp|P54320|ELN_MOUSE Elastin OS=Mus musculus GN=Eln PE $=2$ SV=2

$>$ sp|P70404|IDHG1_MOUSE Isocitrate dehydrogenase [NAD] subunit gamma 1, mitochondrial OS=M sp|P63038|CH60_MOUSE $60 \mathrm{kDa}$ heat shock protein, mitochondrial OS=Mus musculus GN=Hspd1 P $>$ sp|P08249|MDHM_MOUSE Malate dehydrogenase, mitochondrial OS=Mus musculus GN=Mdh2 PE= $>$ sp|Q9EQ20|MMSA_MOUSE Methylmalonate-semialdehyde dehydrogenase [acylating], mitochondria $>$ sp|P70296|PEBP1_MOUSE Phosphatidylethanolamine-binding protein 1 OS=Mus musculus GN=PebF $>$ sp|Q3UV70|PDP1_MOUSE [Pyruvate dehydrogenase [acetyl-transferring]]-phosphatase 1, mitochond sp|Q5SWU9|ACACA_MOUSE Acetyl-CoA carboxylase 1 OS=Mus musculus GN=Acaca PE=1 SV=1 sp|Q9Z2I9|SUCB1_MOUSE Succinyl-CoA ligase [ADP-forming] subunit beta, mitochondrial OS=Mu sp|Q91VM9-2|IPYR2_MOUSE Isoform 2 of Inorganic pyrophosphatase 2, mitochondrial OS=Mus mu $>$ SP|P17156|HSP72 MOUSE Heat shock-related $70 \mathrm{kDa}$ protein $2 \mathrm{OS}=$ Mus musculus $\mathrm{GN}=\mathrm{Hspa2} \mathrm{PE}=1$ $>$ sp|O55028|BCKD_MOUSE [3-methyl-2-oxobutanoate dehydrogenase [lipoamide]] kinase, mitochond $>$ sp|O55028|BCKD_MOUSE [3-methyl-2-oxobutanoate dehydrogenase [lipoamide]] kinase, mitochond $>$ sp|Q9QUJ7|ACSL4_MOUSE Long-chain-fatty-acid--CoA ligase 4 OS=Mus musculus GN=Acsl4 PE= $>$ sp|Q9QUJ7|ACSL4_MOUSE Long-chain-fatty-acid--CoA ligase $4 \mathrm{OS}=$ Mus musculus $\mathrm{GN}=\mathrm{Acsl} 4 \mathrm{PE}=$ $>$ sp|P51660|DHB4_MOUSE Peroxisomal multifunctional enzyme type 2 OS=Mus musculus GN=Hsd17 $>$ sp|P85094|ISC2A_MOUSE Isochorismatase domain-containing protein 2A, mitochondrial OS=Mus $>$ sp|P51175|PPOX_MOUSE Protoporphyrinogen oxidase OS=Mus musculus GN=Ppox PE=2 SV=1 $>$ sp|Q64433|CH10_MOUSE $10 \mathrm{kDa}$ heat shock protein, mitochondrial OS=Mus musculus GN=Hspe1 P $>$ sp|Q61462|CY24A_MOUSE Cytochrome b-245 light chain OS=Mus musculus $\mathrm{GN}=\mathrm{Cyba} \mathrm{PE}=1 \mathrm{SV}=3$

$>$ sp|Q9D051|ODPB_MOUSE Pyruvate dehydrogenase E1 component subunit beta, mitochondrial OS=A
204 >sp|O08579|EMD_MOUSE Emerin OS=Mus musculus GN=Emd PE=1 SV=1

$.206>$ sp|Q64727|VINC_MOUSE Vinculin OS=Mus musculus GN=Vcl PE $=1 \mathrm{SV}=4$

0.209 >sp|Q9D898|ARP5L_MOUSE Actin-related protein 2/3 complex subunit 5-like protein OS=M

$.211>$ >sp|Q61233|PLSL_MOUSE Plastin-2 OS=Mus musculus GN=Lcp1 PE=1 SV=4

214 >sp|Q9WV32|ARC1B_MOUSE Actin-related protein 2/3 complex subunit 1B OS=Mus mus

0.216 >sp|Q8CC35|SYNPO_MOUSE Synaptopodin OS=Mus musculus GN=Synpo PE=1 SV=2

$219>$ sp|P18760|COF1_MOUSE Cofilin-1 OS=Mus musculus GN=Cfl1 PE=1 SV=3

$0.221>$ sp|Q9CPW4|ARPC5_MOUSE Actin-related protein 2/3 complex subunit 5 OS=Mus musc

$223>$ sp|Q0GNC1|INF2_MOUSE Inverted formin-2 OS=Mus musculus GN=Inf2 PE=1 SV=1 26 >sp|P49813|TMOD1_MOUSE Tropomodulin-1 OS=Mus musculus GN=Tmod1 PE=2 SV=2

> >sp|P21107|TPM3_MOUSE Tropomyosin alpha-3 chain OS=Mus musculus GN=Tpm3 PE=1 $>$ tr|Q3U0Q9|Q3U0Q9 MOUSE Putative uncharacterized protein (Fragment) OS=Mus muscil 作 $0.236>\operatorname{tr} \mid$ A1BN54|A1BN54_MOUSE Alpha actinin 1a OS=Mus musculus GN=Actn1 $\mathrm{PE}=2 \mathrm{SV}=1$

$0.238>$ sp|Q9R0P5|DEST_MOUSE Destrin OS=Mus musculus GN=Dstn $\mathrm{PE}=1 \mathrm{SV}=3$

$0.241>$ >sp|O08808|DIAP1_MOUSE Protein diaphanous homolog 1 OS=Mus musculus GN=Diaph1 $0.243>$ >sp|Q7TMB8|CYFP1_MOUSE Cytoplasmic FMR1-interacting protein 1 OS=Mus musculus $246>$ sp|P97434|MPRIP_MOUSE Myosin phosphatase Rho-interacting protein OS=Mus musculus $0.248>$ sp|P59999|ARPC4_MOUSE Actin-related protein 2/3 complex subunit 4 OS=Mus musculus 
$>$ sp|P35486|ODPA_MOUSE Pyruvate dehydrogenase E1 component subunit alpha, somatic form, mitoc $>$ sp|P50544|ACADV_MOUSE Very long-chain specific acyl-CoA dehydrogenase, mitochondrial OS=N sp|P26638|SYSC_MOUSE Serine--tRNA ligase, cytoplasmic OS=Mus musculus GN=Sars PE=2 SV= $>$ sp|O35855|BCAT2_MOUSE Branched-chain-amino-acid aminotransferase, mitochondrial OS=Mus m $>$ sp|P56382|ATP5E_MOUSE ATP synthase subunit epsilon, mitochondrial OS=Mus musculus GN=Atp $>$ sp|Q3U9G9|LBR_MOUSE Lamin-B receptor OS=Mus musculus GN=Lbr $\mathrm{PE}=1 \mathrm{SV}=2$

$>$ sp|Q2TPA8|HSDL2_MOUSE Hydroxysteroid dehydrogenase-like protein 2 OS=Mus musculus GN=H $>$ sp|Q9CZW5|TOM70_MOUSE Mitochondrial import receptor subunit TOM70 OS=Mus musculus GN sp|Q8C0V9|FRMD6_MOUSE FERM domain-containing protein 6 OS=Mus musculus GN=Frmd6 PE sp|Q8BHC4|DCAKD_MOUSE Dephospho-CoA kinase domain-containing protein OS=Mus musculus sp|Q9DBF1|AL7A1_MOUSE Alpha-aminoadipic semialdehyde dehydrogenase OS=Mus musculus GP $>$ sp|Q9DAR7|DCPS_MOUSE m7GpppX diphosphatase OS=Mus musculus GN=Dcps PE=1 SV=1

$>$ sp|P84091|AP2M1_MOUSE AP-2 complex subunit mu OS=Mus musculus GN=Ap2m1 PE=1 SV=1 $>$ sp|Q99LC5|ETFA_MOUSE Electron transfer flavoprotein subunit alpha, mitochondrial OS=Mus musc $>$ sp|O88587|COMT_MOUSE Catechol O-methyltransferase OS=Mus musculus GN=Comt PE=1 SV=2 $>$ sp|Q9JK48|SHLB1_MOUSE Endophilin-B1 OS=Mus musculus GN=Sh3glb1 PE=1 SV=1

sp|Q8K1M6|DNM1L_MOUSE Dynamin-1-like protein OS=Mus musculus GN=Dnm1l PE=1 SV=2

sp|Q99LD8|DDAH2_MOUSE N(G),N(G)-dimethylarginine dimethylaminohydrolase 2 OS=Mus musc $>$ sp|Q9D8B4|NDUAB_MOUSE NADH dehydrogenase [ubiquinone] 1 alpha subcomplex subunit 11 Os $>$ sp|P28271|ACOC MOUSE Cytoplasmic aconitate hydratase OS=Mus musculus GN=Aco1 PE=1 SV= $>$ sp|O35857|TIM44_MOUSE Mitochondrial import inner membrane translocase subunit TIM44 OS=M $>$ sp|Q9Z1J3|NFS1_MOUSE Cysteine desulfurase, mitochondrial OS=Mus musculus GN=Nfs1 PE=2 S sp|P16460|ASSY_MOUSE Argininosuccinate synthase OS=Mus musculus GN=Ass1 PE=1 SV=1 sp|O35683|NDUA1_MOUSE NADH dehydrogenase [ubiquinone] 1 alpha subcomplex subunit 1 OS= $>$ sp|Q9JHR7|IDE_MOUSE Insulin-degrading enzyme OS=Mus musculus GN=Ide PE=1 SV=1 $>$ sp|Q99LX0|PARK7_MOUSE Protein DJ-1 OS=Mus musculus GN=Park7 PE=1 SV=1

$>$ sp|Q8CHT0|AL4A1_MOUSE Delta-1-pyrroline-5-carboxylate dehydrogenase, mitochondrial OS=Mus $>$ sp|Q9CZU6|CISY_MOUSE Citrate synthase, mitochondrial OS=Mus musculus GN=Cs $P E=1 \mathrm{SV}=1$ $>$ sp|P03930|ATP8_MOUSE ATP synthase protein 8 OS=Mus musculus GN=Mtatp8 PE=1 SV=1 sp|Q9DCC8|TOM20_MOUSE Mitochondrial import receptor subunit TOM20 homolog OS=Mus musc sp|Q60936|ADCK3_MOUSE Chaperone activity of bc1 complex-like, mitochondrial OS=Mus muscul $>$ sp|Q9Z2Y8|PROSC_MOUSE Proline synthase co-transcribed bacterial homolog protein OS=Mus mus $>$ SP|A2ARZ3|FSIP2 MOUSE Fibrous sheath-interacting protein $2 \mathrm{OS}=$ Mus musculus GN=Fsip2 $\mathrm{PE}=1$ sp|Q91XE0|GLYAT_MOUSE Glycine N-acyltransferase OS=Mus musculus GN=Glyat $>$ sp|Q9QZD8|DIC_MOUSE Mitochondrial dicarboxylate carrier OS=Mus musculus GN=Slc25a10 PE sp|P11352|GPX1_MOUSE Glutathione peroxidase 1 OS=Mus musculus GN=Gpx1 PE=1 SV=2 sp|P06801|MAOX_MOUSE NADP-dependent malic enzyme OS=Mus musculus GN=Me1 PE=1 SV= sp|P47740|AL3A2_MOUSE Fatty aldehyde dehydrogenase OS=Mus musculus GN=Aldh3a2 PE=2 SV $>$ sp|Q60597|ODO1_MOUSE 2-oxoglutarate dehydrogenase, mitochondrial OS=Mus musculus GN=Ogc $>$ SP|Q9CWSO|DDAH1_MOUSE N(G) N(G)-dimethylarginine dimethylaminohydrolase 1 OS=Mus mus $>$ sp|Q9Z2I8|SUCB2_MOUSE Succinyl-CoA ligase [GDP-forming] subunit beta, mitochondrial OS=M $>$ sp|P42859|HD_MOUSE Huntingtin OS=Mus musculus GN=Htt $\mathrm{PE}=1 \mathrm{SV}=2$

sp|Q9CZ42|NNRD_MOUSE ATP-dependent (S)-NAD(P)H-hydrate dehydratase OS=Mus musculus $\mathrm{C}$ sp|Q9Z1Q5|CLIC1_MOUSE Chloride intracellular channel protein $1 \mathrm{OS}=$ Mus musculus GN=Clic1 PE sp|P55096|ABCD3_MOUSE ATP-binding cassette sub-family D member 3 OS=Mus musculus GN=A $>$ sp $\mid$ P16125|LDHB MOUSE L-lactate dehydrogenase $B$ chain OS=Mus musculus GN=Ldhb $P E=1 \mathrm{SV}=$ $>$ sp|Q9QUI0|RHOA_MOUSE Transforming protein RhoA OS=Mus musculus GN=Rhoa PE=1 SV=1 $>$ sp|O70325|GPX41_MOUSE Phospholipid hydroperoxide glutathione peroxidase, mitochondrial OS=N $>$ sp|P63085|MK01_MOUSE Mitogen-activated protein kinase 1 OS=Mus musculus GN=Mapk1 PE=1 $\begin{array}{ll}-0.349 & 0.325 \\ -0.340 & 0.327\end{array}$ $\begin{array}{rr}-0.340 & 0.327 \\ -0.338 & 0.330\end{array}$ $0.340>$ > 0.342
sp|Q6IRU2|TPM4_MOUSE Tropomyosin alpha-4 chain OS=Mus musculus GN=Tpm4 PE
sp|O89053|COR1A_MOUSE Coronin-1A OS=Mus musculus GN=Coro1a $P E=1 \mathrm{SV}=5$ sp|O89053|COR1A_MOUSE Coronin-1A OS=Mus musculus GN=Coro1a $\mathrm{PE}=1 \mathrm{SV}=5$ $>$ sp|P84075|HPCA_MOUSE Neuron-specific calcium-binding protein hippocalcin OS=Mus $\mathrm{m}$ $>$ sp|P68510|1433F_MOUSE 14-3-3 protein eta OS=Mus musculus GN=Ywhah $\mathrm{PE}=1 \mathrm{SV}=2$ $>$ sp|Q99L88|SNTB1_MOUSE Beta-1-syntrophin OS=Mus musculus GN=Sntb1 PE=1 SV=4 $>$ sp|Q99L88|SNTB1_MOUSE Beta-1-syntrophin OS=Mus musculus GN=Sntb1 PE=1
$>$ sp|Q02566|MYH6_MOUSE Myosin-6 OS=Mus musculus GN=Myh6 PE=1 SV=2 Cell adhesion GO:0007155 0.347

Q61282|PGCA_MOUSE Aggrecan core protein OS=Mus musculus GN=Acan PE=1 SV

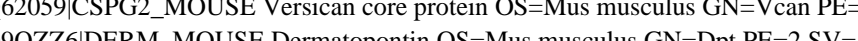
P29788|VTNC_MOUSE Vitronectin OS=Mus musculus GN=Vtn $P E=1 \mathrm{SV}=2$ P3906_/NPNT_MOUSE Nephronectin OS=Mus musculus GN=Npnt PE=1 SV=1 |P21956|MFGM_MOUSE Lactadherin OS=Mus musculus GN=Mfge8 PE=1 SV=3 O88207|CO5A1_MOUSE Collagen alpha-1(V) chain OS=Mus musculus GN=Col5a1 PE

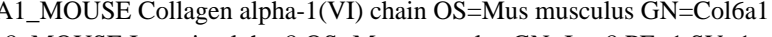
Q640N1|AEBP1_MOUSE Adipocyte enhancer-binding protein $1 \mathrm{OS}=$ Mus musculus GN
$\mathrm{P} 12023$ |A4 MOUSE Amyloid beta A4 protein OS=Mus musculus GN=App PE $=1 \mathrm{SV}=3$ A6H584|CO6A5_MOUSE Collagen alpha-5(VI) chain OS=Mus musculus GN=Col6a5 PE Q62009|POSTN_MOUSE Periostin OS=Mus musculus GN=Postn $P E=1 \mathrm{SV}=2$ Q61879|MYH10_MOUSE Myosin-10 OS=Mus musculus GN=Myh10 PE $=1 \mathrm{SV}=2$ QQ99K41|EMIL1_MOUSE EMILIN-1 OS=Mus musculus GN=Emilin1 PE=1 SV=1 P26039|TLN1_MOUSE Tidogen-2 OS=Mus musculus GN=Nid2 PE $=1 \mathrm{SV}=2$ Q8BS97|Q8BS97_MOUSE Versican core protein OS=Mus musculus GN=Vcan PE=2 |Q62470|ITA3_MOUSE Integrin alpha-3 OS=Mus musculus GN=Itga3 PE=1 SV=1 Q61490|CD166_MOUSE CD166 antigen OS=Mus musculus GN=Alcam PE=1 SV=3 Q8VI36|PAXI_MOUSE Paxillin OS=Mus musculus GN=Pxn PE=1 SV=1

$\mathrm{Zq}$


$>$ sp|P50431|GLYC_MOUSE Serine hydroxymethyltransferase, cytosolic OS=Mus musculus GN=Shmt $>$ sp|Q9QUM9|PSA6_MOUSE Proteasome subunit alpha type-6 OS=Mus musculus GN=Psma6 PE=1 S $>$ sp|P31750|AKT1_MOUSE RAC-alpha serine/threonine-protein kinase OS=Mus musculus GN=Akt1 $>$ sp|Q8BVI4|DHPR_MOUSE Dihydropteridine reductase OS=Mus musculus GN=Qdpr PE=1 SV=2 $>$ sp|Q9CQW1|YKT6_MOUSE Synaptobrevin homolog YKT6 OS=Mus musculus GN=Ykt6 PE=2 SV= $>$ sp|P56380|AP4A_MOUSE Bis(5'-nucleosyl)-tetraphosphatase [asymmetrical] OS=Mus musculus GN= $>$ sp|Q07417|ACADS MOUSE Short-chain specific acyl-CoA dehydrogenase, mitochondrial OS=Mus n $>$ sp|Q61425|HCDH_MOUSE Hydroxyacyl-coenzyme A dehydrogenase, mitochondrial OS=Mus muscu Sp|Q9D0I9|SYRC_MOUSE Arginine--tRNA ligase, cytoplasmic OS=Mus musculus GN=Rars PE=2 S sp|Q8BMF4|ODP2_MOUSE Dihydrolipoyllysine-residue acetyltransferase component of pyruvate deh sp|Q8BGB8|COQ4_MOUSE Ubiquinone biosynthesis protein COQ4 homolog, mitochondrial OS=Mu $>$ sp|Q91VD9|NDUS1_MOUSE NADH-ubiquinone oxidoreductase $75 \mathrm{kDa}$ subunit, mitochondrial OS= sp|Q9CRB9|CHCH3 MOUSE Coiled-coil-helix-coiled-coil-helix domain-containing protein 3, mitoch $>\operatorname{tr}|\mathrm{Q} 3 \mathrm{U} 1 \mathrm{C} 4| \mathrm{Q} 3 \mathrm{U} 1 \mathrm{C} 4 \_$MOUSE MCG1271 OS=Mus musculus GN=Secisbp2 PE=2 SV=1

$>$ sp|Q9DCX2|ATP5H_MOUSE ATP synthase subunit d, mitochondrial OS=Mus musculus GN=Atp5h tr|Q99N15|Q99N15_MOUSE 17beta-hydroxysteroid dehydrogenase type 10/short chain L-3-hydroxya sp|Q9D6J5|NDUB8_MOUSE NADH dehydrogenase [ubiquinone] 1 beta subcomplex subunit 8, mitoc sp|Q8BKZ9|ODPX_MOUSE Pyruvate dehydrogenase protein X component, mitochondrial OS=Mus n $>$ sp|Q8BGT8|PHIPL_MOUSE Phytanoyl-CoA hydroxylase-interacting protein-like OS=Mus musculus $>$ sp|Q91WD5|NDUS2 MOUSE NADH dehydrogenase [ubiquinone] iron-sulfur protein 2, mitochondri $>$ sp|Q8CAY6|THIC_MOUSE Acetyl-CoA acetyltransferase, cytosolic OS=Mus musculus GN=Acat2 PI $>$ sp|Q8BIJ6|SYIM_MOUSE Isoleucine--tRNA ligase, mitochondrial OS=Mus musculus GN=Iars2 PE=: sp|P51174|ACADL_MOUSE Long-chain specific acyl-CoA dehydrogenase, mitochondrial OS=Mus n sp|P08228|SODC_MOUSE Superoxide dismutase [Cu-Zn] OS=Mus musculus GN=Sod1 PE=1 SV=2 sp|Q99LP6|GRPE1_MOUSE GrpE protein homolog 1, mitochondrial OS=Mus musculus GN=Grpel1 $>$ sp|P80313|TCPH_MOUSE T-complex protein 1 subunit eta $\mathrm{OS}=$ Mus musculus $\mathrm{GN}=\mathrm{Cct} 7 \mathrm{PE}=1 \mathrm{SV}=1$ 7 75 MOUSE Stress-70 protein, mitochondrial OS=Mus musculus GN=Hspa9 PE=1 SV $>$ sp|P63168|DYL1_MOUSE Dynein light chain 1, cytoplasmic OS=Mus musculus GN=Dynll1 PE=1 S sp|Q9DBM2|ECHP_MOUSE Peroxisomal bifunctional enzyme OS=Mus musculus GN=Ehhadh PE=1 sp|P43024|CX6A1_MOUSE Cytochrome c oxidase subunit 6A1, mitochondrial OS=Mus musculus GI sp|Q9CXW2|RT22_MOUSE 28S ribosomal protein S22, mitochondrial OS=Mus musculus GN=Mrps sp|Q9DC70|NDUS7_MOUSE NADH dehydrogenase [ubiquinone] iron-sulfur protein 7, mitochondria $>$ sp|Q9Z1Z2|STRAP_MOUSE Serine-threonine kinase receptor-associated protein OS=Mus musculus $\mathrm{C}$ (CPT2_MOUSE Carnitine O-palmitoyltransferase 2, mitochondrial OS=Mus musculus GN $>$ sp $\mid$ P47934|CACP_MOUSE Carnitine O-acetyltransferase OS=Mus musculus GN=Crat $P E=1$ SV=3 $>$ sp|Q9CQH3|NDUB5_MOUSE NADH dehydrogenase [ubiquinone] 1 beta subcomplex subunit 5, mito sp|P56480|ATPB_MOUSE ATP synthase subunit beta, mitochondrial OS=Mus musculus GN=Atp5b sp|Q63844|MK03_MOUSE Mitogen-activated protein kinase 3 OS=Mus musculus GN=Mapk3 PE=1 $>$ sp|P53395|ODB2_MOUSE Lipoamide acyltransferase component of branched-chain alpha-keto acid d $>$ sp|P28352|APEX1_MOUSE DNA-(apurinic or apyrimidinic site) lyase OS=Mus musculus GN=Apex1 $>$ sp|Q64521|GPDM_MOUSE Glycerol-3-phosphate dehydrogenase, mitochondrial OS=Mus musculus C $>$ sp|Q8VCL2|SCO2_MOUSE Protein SCO2 homolog, mitochondrial OS=Mus musculus GN=Sco2 PE= $>$ sp|Q8BFR5|EFTU_MOUSE Elongation factor Tu, mitochondrial OS=Mus musculus GN=Tufm PE=1 sp|P62492|RB11A_MOUSE Ras-related protein Rab-11A OS=Mus musculus GN=Rab11a PE=1 SV= sp|Q9D855|QCR7_MOUSE Cytochrome b-c1 complex subunit 7 OS=Mus musculus GN=Uqcrb PE=1 $>$ sp|O70378|EMC8_MOUSE ER membrane protein complex subunit 8 OS=Mus musculus GN=Emc8 P SP|Q9Z0X1|AIFM1_MOUSE Apoptosis-inducing factor 1 mitochondrial OS=Mus musculus $\mathrm{GN}=\mathrm{A}$

(
$-0.015$

$-0.011 \quad 0.448$
sp|Q61292|LAMB2_MOUSE Laminin subunit beta-2 OS=Mus musculus GN=Lamb2 PE=2 sp|Q02788|CO6A2_MOUSE Collagen alpha-2(VI) chain OS=Mus musculus GN=Col6a2 PE tr|Q91XX7|Q91XX7_MOUSE Protocadherin gamma B2 OS=Mus musculus GN=Pcdhgb2 P Sp|Q80YX1|TENA_MOUSE Tenascin OS=Mus musculus GN=Tnc PE $=1 \mathrm{SV}=1$

$>$ sp|Q9WVH9|FBLN5_MOUSE Fibulin-5 OS=Mus musculus GN=Fbln5 PE=2 SV=1

tr|B8JK39|B8JK39_MOUSE Integrin alpha 9 OS=Mus musculus GN=Itga9 PE=3 SV=1

tr|B9EHT6|B9EHT6_MOUSE Fn1 protein OS=Mus musculus GN=Fn1 PE=2 SV=1

tr|F8VQJ3|F8VQJ3_MOUSE Laminin subunit gamma-1 OS=Mus musculus GN=Lamc1 PE sp|P62746|RHOB_MOUSE Rho-related GTP-binding protein RhoB OS=Mus musculus GN $>$ sp|Q9EPC1|PARVA_MOUSE Alpha-parvin OS=Mus musculus GN=Parva PE=1 SV=1 sp|P26231|CTNA1_MOUSE Catenin alpha-1 OS=Mus musculus GN=Ctnna1 PE=1 SV=1 sp|Q3UTJ2|SRBS2_MOUSE Sorbin and SH3 domain-containing protein 2 OS=Mus musculu $>\mathrm{sp}|\mathrm{Q} 8 \mathrm{BFW7}| \mathrm{LPP}$ MOUSE Lipoma-preferred partner homolog OS=Mus musculus GN=Lpp sp|Q8CIZ8|VWF_MOUSE von Willebrand factor OS=Mus musculus GN=Vwf PE $=1 \mathrm{SV}=2$ sp|Q9Z0M6|CD97_MOUSE CD97 antigen OS=Mus musculus GN=Cd97 PE=1 SV=2 $>$ sp|Q71FD7|FBLI1_MOUSE Filamin-binding LIM protein 1 OS=Mus musculus GN=Fblim1 sp|Q9QUP5|HPLN1_MOUSE Hyaluronan and proteoglycan link protein $1 \mathrm{OS}=$ Mus muscul $>$ sp|Q9WTR5|CAD13_MOUSE Cadherin-13 OS=Mus musculus GN=Cdh13 PE=1 SV=2 sp|O70309|ITB5_MOUSE Integrin beta- 5 OS=Mus musculus GN=Itgb5 PE=1 SV=2 $>$ sp|Q64727|VINC_MOUSE Vinculin OS=Mus musculus GN=Vcl PE $=1 \mathrm{SV}=4$ sp|P02468|LAMC1_MOUSE Laminin subunit gamma-1 OS=Mus musculus GN=Lamc1 PEsp|Q8R2Y2|MUC18_MOUSE Cell surface glycoprotein MUC18 OS=Mus musculus GN=M tr|A2RS43|A2RS43_MOUSE Protein Pcdh7 OS=Mus musculus GN=Pcdh7 PE=2 SV=1 $>$ sp|Q61735|CD47_MOUSE Leukocyte surface antigen CD47 OS=Mus musculus GN=Cd47 sp|Q9D1H9|MFAP4_MOUSE Microfibril-associated glycoprotein 4 OS=Mus musculus GN PD|Q8BZF8|PGM5_MOUSE Phosphoglucomutase-like protein $5 \mathrm{OS}=$ Mus musculus GN=P $>\mathrm{sp} \mid \mathrm{P97300|}$ |NPTN_MOUSE Neuroplastin OS=Mus musculus GN=Nptn PE=1 SV=3

sp|Q08481|PECA1_MOUSE Platelet endothelial cell adhesion molecule OS=Mus musculus sp|P14206|RSSA_MOUSE 40S ribosomal protein SA OS=Mus musculus GN=Rpsa PE=1 S $>$ sp|P29533|VCAM1_MOUSE Vascular cell adhesion protein 1 OS=Mus musculus GN=Vca tr|E9QB01|E9QB01_MOUSE Neural cell adhesion molecule $1 \mathrm{OS}=$ Mus musculus GN=Nca sp|P30999|CTND1_MOUSE Catenin delta-1 OS=Mus musculus GN=Ctnnd1 PE=1 SV=2 $>$ sp|P35441|TSP1_MOUSE Thrombospondin-1 OS=Mus musculus GN=Thbs1 $\mathrm{PE}=1 \mathrm{SV}=$ sp|Q99JY8|LPP3_MOUSE Lipid phosphate phosphohydrolase 3 OS=Mus musculus GN=Pp sp|P82198|BGH3 MOUSE Transforming growth factor-beta-induced protein ig-h3 OS=Mus sp|Q61330|CNTN2_MOUSE Contactin-2 OS=Mus musculus GN=Cntn2 PE=2 SV=2 sp|O70423|AOC3_MOUSE Membrane primary amine oxidase OS=Mus musculus GN=Aoc sp|Q9QUIO|RHOA_MOUSE Transforming protein RhoA OS=Mus musculus GN=Rhoa PE= $>$ sp|Q60847|COCA1_MOUSE Collagen alpha-1(XII) chain OS=Mus musculus GN=Col12a1 $>$ sp|P35330|ICAM2_MOUSE Intercellular adhesion molecule 2 OS=Mus musculus GN=Ican sp|Q8VCC9|SPON1_MOUSE Spondin-1 OS=Mus musculus GN=Spon1 PE=2 SV=1 sp|O54890|ITB3_MOUSE Integrin beta-3 OS=Mus musculus GN=Itgb3 PE=1 SV=2 sp|P10493|NID1_MOUSE Nidogen-1 OS=Mus musculus GN=Nid1 PE=1 SV=2 $>$ sp|Q60634|FLOT2_MOUSE Flotillin-2 OS=Mus musculus GN=Flot2 PE=1 SV=2

$0.557>$ sp|P13595|NCAM1_MOUSE Neural cell adhesion molecule 1 OS=Mus musculus GN=Ncan $0.559>$ >sp $\mid$ P40240|CD9_MOUSE CD9 antigen OS=Mus musculus GN=Cd9 PE=1 SV=2 $0.562>$ tr| G3UW60|G3UW60_MOUSE MCG14773 OS=Mus musculus GN=Susd5 PE=4 SV=1

$0.564 \mid>$ sp|Q60675|LAMA2_MOUSE Laminin subunit alpha-2 OS=Mus musculus GN=Lama2 PE=1 
$>$ tr|Q8JZU2|Q8JZU2 MOUSE Protein Slc25a1 OS=Mus musculus GN=Slc25a1 PE=2 SV=1 sp|Q9Z1X4|ILF3_MOUSE Interleukin enhancer-binding factor 3 OS=Mus musculus GN=Ilf3 PE=1 S sp|Q5SSK3|TEFM_MOUSE Transcription elongation factor, mitochondrial OS=Mus musculus GN=T tr|Q924B0|Q924B0_MOUSE Inositol (Myo)-1(Or 4)-monophosphatase 1 OS=Mus musculus GN=Imp $>$ sp|Q9QYB1|CLIC4_MOUSE Chloride intracellular channel protein 4 OS=Mus musculus GN=Clic4 Pl $>$ sp|Q6ZWQ0|SYNE2_MOUSE Nesprin-2 OS=Mus musculus GN=Syne2 PE=1 SV=2

$>$ sp|P25688|URIC_MOUSE Uricase OS=Mus musculus GN=Uox PE $=1 \mathrm{SV}=2$

sp|Q9DCM0|ETHE1_MOUSE Protein ETHE1, mitochondrial OS=Mus musculus GN=Ethe1 PE=1 SV $>$ sp|Q91V92|ACLY_MOUSE ATP-citrate synthase OS=Mus musculus GN=Acly PE=1 SV=1 sp|P52480|KPYM_MOUSE Pyruvate kinase isozymes M1/M2 OS=Mus musculus GN=Pkm PE=1 SV $>$ sp|Q99JR1|SFXN1_MOUSE Sideroflexin-1 OS=Mus musculus GN=Sfxn1 PE=1 SV=3

$>$ sp|O08749|DLDH_MOUSE Dihydrolipoyl dehydrogenase, mitochondrial OS=Mus musculus GN=Dld $>$ sp|Q8VEM8|MPCP_MOUSE Phosphate carrier protein, mitochondrial OS=Mus musculus GN=Slc25a $>$ sp|Q922W5|P5CR1_MOUSE Pyrroline-5-carboxylate reductase 1, mitochondrial OS=Mus musculus $C$ $>$ sp|Q8CH40|NUDT6_MOUSE Nucleoside diphosphate-linked moiety X motif 6 OS=Mus musculus GP sp|Q9D3D9|ATPD_MOUSE ATP synthase subunit delta, mitochondrial OS=Mus musculus GN=Atp5 sp|P56395|CYB5_MOUSE Cytochrome b5 OS=Mus musculus GN=Cyb5a PE=1 SV=2

sp|Q9WTP6|KAD2_MOUSE Adenylate kinase 2, mitochondrial OS=Mus musculus GN=Ak2 PE=1 S $>$ sp|Q99J99|THTM_MOUSE 3-mercaptopyruvate sulfurtransferase OS=Mus musculus GN=Mpst PE=1 $>$ sp|Q921H8|THIKA_MOUSE 3-ketoacyl-CoA thiolase A, peroxisomal OS=Mus musculus GN=Acaa1 sp|P17665|COX7C_MOUSE Cytochrome c oxidase subunit 7C, mitochondrial OS=Mus musculus GN $>$ sp|Q9D3P8|PLRKT_MOUSE Plasminogen receptor (KT) OS=Mus musculus GN=Plgrkt PE=1 SV= sp|Q8BH04|PCKGM_MOUSE Phosphoenolpyruvate carboxykinase [GTP], mitochondrial OS=Mus m sp|P28474|ADHX_MOUSE Alcohol dehydrogenase class-3 OS=Mus musculus GN=Adh5 PE=1 SV= sp|P62259|1433E_MOUSE 14-3-3 protein epsilon OS=Mus musculus GN=Ywhae $\mathrm{PE}=1 \mathrm{SV}=1$

$>$ sp|Q9D6K8|FUND2 MOUSE FUN14 domain-containing protein $2 \mathrm{OS}=$ Mus musculus GN=Fundc2 PI $>$ sp|Q9R112|SQRD_MOUSE Sulfide:quinone oxidoreductase, mitochondrial OS=Mus musculus GN=S sp|P19096|FAS_MOUSE Fatty acid synthase $\mathrm{OS}=$ Mus musculus GN=Fasn $\mathrm{PE}=1 \mathrm{SV}=2$

$>$ sp|Q9CQX2|CYB5B_MOUSE Cytochrome b5 type B OS=Mus musculus GN=Cyb5b PE=1 SV=1 $>$ sp|Q64105|SPRE_MOUSE Sepiapterin reductase OS=Mus musculus GN=Spr PE=1 SV=1

sp|Q8VHJ7|PRGC2_MOUSE Peroxisome proliferator-activated receptor gamma coactivator 1-beta OS sp|Q9WUM5|SUCA_MOUSE Succinyl-CoA ligase [ADP/GDP-forming] subunit alpha, mitochondrial $>$ tr|Q91VA7|Q91VA7_MOUSE Isocitrate dehydrogenase $3(\mathrm{NAD}+)$ beta OS=Mus musculus GN=Idh3b $>$ sp|P26443|DHE3_MOUSE Glutamate dehydrogenase 1, mitochondrial OS=Mus musculus GN=Glud1 >sp|Q9QZH6|ECSIT_MOUSE Evolutionarily conserved signaling intermediate in Toll pathway, mitoch $>$ sp|P37040|NCPR_MOUSE NADPH--cytochrome P450 reductase OS=Mus musculus GN=Por PE=1 S $>$ sp|O09005|DEGS1_MOUSE Sphingolipid delta(4)-desaturase DES1 OS=Mus musculus GN=Degs1 P sp|P08074|CBR2_MOUSE Carbonyl reductase [NADPH] 2 OS=Mus musculus GN=Cbr2 PE=1 SV=1 $>$ sp|Q9D7B6|ACAD8_MOUSE Isobutyryl-CoA dehydrogenase, mitochondrial OS=Mus musculus GN= $>$ sp|P35700|PRDX1_MOUSE Peroxiredoxin-1 OS=Mus musculus GN=Prdx1 PE=1 SV=1

$>$ sp|Q9QYR9|ACOT2_MOUSE Acyl-coenzyme A thioesterase 2, mitochondrial OS=Mus musculus GN $>$ sp|Q9D6R2|IDH3A_MOUSE Isocitrate dehydrogenase [NAD] subunit alpha, mitochondrial OS=Mus I $>$ sp|O89023|TPP1_MOUSE Tripeptidyl-peptidase 1 OS=Mus musculus GN=Tpp1 PE=1 SV=2 $>$ sp|Q60932|VDAC1_MOUSE Voltage-dependent anion-selective channel protein 1 OS=Mus musculus $>$ sp|Q9CPY7|AMPL_MOUSE Cytosol aminopeptidase OS=Mus musculus GN=Lap3 PE=1 SV=3 $>$ sp|P36552|HEM6_MOUSE Coproporphyrinogen-III oxidase, mitochondrial OS=Mus musculus GN=C $>$ sp|Q9CQN1|TRAP1_MOUSE Heat shock protein $75 \mathrm{kDa}$, mitochondrial OS=Mus musculus GN=Trap $>$ sp|Q60930|VDAC2_MOUSE Voltage-dependent anion-selective channel protein 2 OS=Mus musculus

$>$ sp|Q8C196|CPSM_MOUSE Carbamoyl-phosphate synthase [ammonia], mitochondrial OS=Mus musc
.567|>sp|Q71LX4|TLN2_MOUSE Talin-2 OS=Mus musculus GN=Tln2 PE=1 SV=3

$>$ sp|P63001|RAC1_MOUSE Ras-relded \begin{tabular}{llll}
$>>$ sp|Q02248|CTNB1_MOUSE Catenin beta-1 OS=Mus musculus GN=Ctnnb1 PE=1 SV=1 & 1.328 & 0.913 \\
\hline
\end{tabular} . 4 >sp|Q61738|ITA7_MOUSE Integrin alpha-7 OS=Mus musculus GN=Itga7 PE=1 SV=3 $\quad \begin{array}{ll}1.587 & 0.934\end{array}$ >sp|O35114|SCRB2_MOUSE Lysosome membrane protein 2 OS=Mus musculus GN=Scarb2 1.649 $>S \mathrm{Q}$ 3V3R 4ITA1_MOUSE Integrin alpha-1 OS=MuS musculus $\mathrm{GN}=\mathrm{Itga} 1 \mathrm{PE}=2 \mathrm{SV}=2$ (1) SPP|Q082 >sp|P14094|AT1B__MOUSE Sodium/potassium-transporting ATPase subunit beta-1 OS=Mus $5.091 \quad 0.97$ \begin{tabular}{l|rrr}
0.586 & $>$ sp|P02469|LAMB1_MOUSE Laminin subunit beta-1 OS=Mus musculus GN=Lamb1 PE=1 $\leqslant$ & 5.615 & 0.985 \\
0.589 & $>$ sp|Q2UY11|COSA1_MOUSE Collagen alpha-1(XXVIII) chain OS=Mus musculus GN=Colz & 12.820 & 0.995 \\
\hline
\end{tabular} 0.591 0.594 Calcium ion binding GO:0005509 0.596

$0.599>$ sp|P70663|SPRL1_MOUSE SPARC-like protein 1 OS=Mus musculus GN=Sparcl1 PE=1 SV

$0.601>>$ sp|Q62059|CSPG2_MOUSE Versican core protein OS=Mus musculus GN=Vcan PE=1 SV= 0.604 >sp|Q8BPB5|FBLN3_MOUSE EGF-containing fibulin-like extracellular matrix protein 1 OS 0.606 >tr|Q6ZWQ9|Q6ZWQ9_MOUSE MCG5400 OS=Mus musculus GN=Myl12a PE=2 SV=1

0.609 >sp|Q91V88|NPNT_MOUSE Nephronectin OS=Mus musculus GN=Npnt PE=1 SV=1

0.611 >sp|Q8K4G1|LTBP4_MOUSE Latent-transforming growth factor beta-binding protein 4 OS= 0.614 >tr|F8WIT2|F8WIT2_MOUSE Annexin OS=Mus musculus GN=Anxa6 PE=3 SV=1

0.616 >sp|Q3THE2|ML12B_MOUSE Myosin regulatory light chain 12B OS=Mus musculus GN=M .619 >sp|Q9D2N4|DTNA_MOUSE Dystrobrevin alpha OS=Mus musculus GN=Dtna PE=1 SV=2 $0.621>$ sp|Q60605|MYL6_MOUSE Myosin light polypeptide 6 OS=Mus musculus GN=Myl6 PE= $0.623>$ sp|P08032|SPTA1_MOUSE Spectrin alpha chain, erythrocytic 1 OS=Mus musculus GN=Spt >sp|Q9R0K7|AT2B2_MOUSE Plasma membrane calcium-transporting ATPase 2 OS=Mus m > >sp|Q9CQ19|MYL9_MOUSE Myosin regulatory light polypeptide 9 OS=Mus musculus GN=$0.631>$ sp|P57780|ACTN4_MOUSE Alpha-actinin-4 OS=Mus musculus GN=Actn4 PE=1 SV=1

$0.633>\operatorname{tr} \mid$ A2AQ53|A2AQ53_MOUSE Fibrillin $1 \mathrm{OS}=$ Mus musculus GN=Fbn1 $\mathrm{PE}=4 \mathrm{SV}=1$

.636 >sp|Q8CI43|MYL6B_MOUSE Myosin light chain 6B OS=Mus musculus GN=Myl6b PE=2 $0.638>\operatorname{tr} \mid$ Q61636|Q61636_MOUSE G-utrophin (Predicted protein) OS=Mus musculus GN=Utrn PE

.643 >tr|Q8C845|Q8C845_MOUSE EF hand domain containing 2 OS=Mus musculus GN=Efhd2 0.646 >sp|P11531|DMD_MOUSE Dystrophin OS=Mus musculus GN=Dmd PE=1 SV=3

0.648 >sp|Q3V0K9|PLSI_MOUSE Plastin-1 OS=Mus musculus GN=Pls1 PE=2 SV=1

$0.651>>s p|Q 08879|$ FBLN1_MOUSE Fibulin-1 OS=Mus musculus GN=Fbln1 PE=1 SV=2

$653>$ sp|O08795|GLU2B_MOUSE Glucosidase 2 subunit beta OS=Mus musculus GN=Prkcsh PE=

sp|P21981|TGM2_MOUSE Protein-glutas 2 subus $0.658>$ sp|O08529|CAN2_MOUSE Calpain-2 catalytic subunit OS=Mus musculus GN=Capn2 PE=2 $0.660>$ sp|P37889|FBLN2_MOUSE Fibulin-2 OS=Mus musculus GN=Fbln2 PE=1 SV=2

$0.663>\operatorname{tr} \mid$ E9Q6R7|E9Q6R7 MOUSE Protein Utrn OS=Mus musculus GN=Utrn PE=4 SV=

0.665 >sp|Q7TPR4|ACTN1_MOUSE Alpha-actinin-1 OS=Mus musculus GN=Actn1 PE=1 SV=1

$0.668>>$ sp|A2ASQ1|AGRIN_MOUSE Agrin OS=Mus musculus GN=Agrn PE=1 SV=1

$0.670>$ sp|P10107|ANXA1_MOUSE Annexin A1 OS=Mus musculus GN=Anxa1 PE=1 SV=2

$0.673>>$ sp|Q07076|ANXA7_MOUSE Annexin A7 OS=Mus musculus GN=Anxa7 PE=2 SV=2

0.675 >sp|P14069|S10A6_MOUSE Protein S100-A6 OS=Mus musculus GN=S100a6 PE=1 SV=3

$0.678>$ sp|Q99K51|PLST_MOUSE Plastin-3 OS=Mus musculus GN=Pls3 PE=1 SV=3

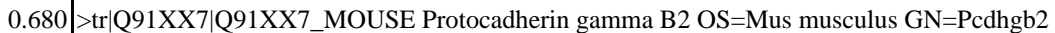

$0.683>$ sp|Q9D4J1|EFHD1_MOUSE EF-hand domain-containing protein D1 OS=Mus musculus GN

$0.685 \mid>$ sp|Q8CG19|LTBP1_MOUSE Latent-transforming growth factor beta-binding protein 1 OS=1

\section{$\mathrm{Zq}$}

rank/N

$\begin{array}{rr}-7.129 & 0.005 \\ -3.806 & 0.014 \\ -3.415 & 0.023\end{array}$

$-3.815$

\begin{tabular}{ll}
-3.415 & 0.023 \\
-3.282 & 0.032 \\
\hline-3.021 & 0.041
\end{tabular} 
$>$ sp|P46471|PRS7_MOUSE 26S protease regulatory subunit 7 OS=Mus musculus GN=Psmc2 PE=1 SV: $>$ sp|P33434|MMP2_MOUSE $72 \mathrm{kDa}$ type IV collagenase OS=Mus musculus GN=Mmp2 PE=2 SV=1 $>$ sp|E9Q557|DESP_MOUSE Desmoplakin OS=Mus musculus GN=Dsp PE=3 SV=1

sp|P06151|LDHA_MOUSE L-lactate dehydrogenase A chain OS=Mus musculus GN=Ldha PE=1 SV= $>$ sp|Q9CR61|NDUB7_MOUSE NADH dehydrogenase [ubiquinone] 1 beta subcomplex subunit 7 OS= PSP21981|TGM2 MOUSE P sp|Q641K1|CBPC1_MOUSE Cytosolic carboxypeptidase 1 OS=Mus musculus GN=Agtpbp1 PE=1 S sp|Q921G7|ETFD_MOUSE Electron transfer flavoprotein-ubiquinone oxidoreductase, mitochondrial C sp|Q8BTZ7|GMPPB_MOUSE Mannose-1-phosphate guanyltransferase beta OS=Mus musculus GN=C sp|O08709|PRDX6_MOUSE Peroxiredoxin-6 OS=Mus musculus GN=Prdx6 PE=1 SV=3

sp|Q9CQZ5|NDUA6_MOUSE NADH dehydrogenase [ubiquinone] 1 alpha subcomplex subunit 6 OS= $>$ sp|Q9WTP7|KAD3 MOUSE GTP:AMP phosphotransferase, mitochondrial OS=Mus musculus GN=A $>$ sp|Q9JLJ2|AL9A1_MOUSE 4-trimethylaminobutyraldehyde dehydrogenase OS=Mus musculus GN= $>$ sp|Q9JIK9|RT34_MOUSE 28S ribosomal protein S34, mitochondrial OS=Mus musculus GN=Mrps34 sp|Q8VCM4|LIPT_MOUSE Lipoyltransferase 1, mitochondrial OS=Mus musculus GN=Lipt1 PE=2 S $>$ sp|Q9D6U8|F162A_MOUSE Protein FAM162A OS=Mus musculus GN=Fam162a PE=2 SV=1

sp|P99029|PRDX5_MOUSE Peroxiredoxin-5, mitochondrial OS=Mus musculus GN=Prdx5 PE=1 SV $>$ sp|Q791V5|MTCH2_MOUSE Mitochondrial carrier homolog 2 OS=Mus musculus GN=Mtch2 PE=1 $>$ sp|Q9CQJ8|NDUB9 MOUSE NADH dehydrogenase [ubiquinone] 1 beta subcomplex subunit 9 OS=N $>$ sp|Q9CQ54|NDUC2_MOUSE NADH dehydrogenase [ubiquinone] 1 subunit C2 OS=Mus musculus G $>$ sp|Q68FD5|CLH1_MOUSE Clathrin heavy chain 1 OS=Mus musculus GN=Cltc PE=1 SV=3

sp|Q9CZW4|ACSL3_MOUSE Long-chain-fatty-acid--CoA ligase 3 OS=Mus musculus GN=Acsl3 PE sp|Q9CQ62|DECR_MOUSE 2,4-dienoyl-CoA reductase, mitochondrial OS=Mus musculus GN=Decr1 $>$ sp $\mid$ P18242|CATD_MOUSE Cathepsin D OS=Mus musculus GN=Ctsd PE=1 SV=1

sp|Q9CXZ1|NDUS4_MOUSE NADH dehydrogenase [ubiquinone] iron-sulfur protein 4, mitochondria $>$ sp|Q62425|NDUA4_MOUSE NADH dehydrogenase [ubiquinone] 1 alpha subcomplex subunit 4 OS= $>$ sp|P47791|GSHR_MOUSE Glutathione reductase, mitochondrial OS=Mus musculus GN=Gsr PE=2 S $>$ sp|Q9Z2Z6|MCAT_MOUSE Mitochondrial carnitine/acylcarnitine carrier protein OS=Mus musculus sp|Q03265|ATPA_MOUSE ATP synthase subunit alpha, mitochondrial OS=Mus musculus GN=Atp5a sp|P63101|1433Z_MOUSE 14-3-3 protein zeta/delta OS=Mus musculus GN=Ywhaz PE=1 SV=1

$>$ sp|Q06890|CLUS_MOUSE Clusterin OS=Mus musculus GN=Clu PE $=1 \mathrm{SV}=1$

$>$ sp|Q61171|PRDX2_MOUSE Peroxiredoxin-2 OS=Mus musculus GN=Prdx2 PE=1 SV=3

$>$ sp|P00405|COX2_MOUSE Cytochrome c oxidase subunit 2 OS=Mus musculus GN=Mtco2 PE=1 SV=

$>$ sp|O88844|IDHC_MOUSE Isocitrate dehydrogenase [NADP] cytoplasmic OS=Mus musculus GN=Idh $>$ sp|Q9WVL0|MAAI_MOUSE Maleylacetoacetate isomerase OS=Mus musculus GN=Gstz1 PE=1 SV= $>$ sp|Q9CYW4|HDHD3_MOUSE Haloacid dehalogenase-like hydrolase domain-containing protein 3 OS sp|P42125|ECI1_MOUSE Enoyl-CoA delta isomerase 1, mitochondrial OS=Mus musculus GN=Eci1 $>$ sp |P10605|CATB_MOUSE Cathepsin B OS=Mus musculus GN=Ctsb PE=1 SV=2

$>$ sp|P15532|NDKA_MOUSE Nucleoside diphosphate kinase A OS=Mus musculus GN=Nme1 PE=1 SV $>$ sp|P29758|OAT_MOUSE Ornithine aminotransferase, mitochondrial OS=Mus musculus GN=Oat PE= $>$ sp|Q8CI78|RMND1_MOUSE Required for meiotic nuclear division protein 1 homolog OS=Mus musc $>$ sp|Q9DCN2|NB5R3_MOUSE NADH-cytochrome b5 reductase 3 OS=Mus musculus GN=Cyb5r3 PE= $>$ sp|Q8R404|QIL1_MOUSE Protein QIL1 OS=Mus musculus GN=Qil1 PE=2 SV=1

sp|Q9QYA2|TOM40_MOUSE Mitochondrial import receptor subunit TOM40 homolog OS=Mus mus sp|Q8VCW8|ACSF2_MOUSE Acyl-CoA synthetase family member 2, mitochondrial OS=Mus muscu SP|Q9CQC7|NDUB4_MOUSE NADH dehydrogenase [ubiquinone] 1 beta subcomplex subunit 4 OS= $>$ sp|P56135|ATPK MOUSE ATP synthase subunit $\mathrm{f}$, mitochondrial OS=Mus musculus GN=Atp5j2 PE

$>$ sp|Q06185|ATP5I_MOUSE ATP synthase subunit e, mitochondrial OS=Mus musculus GN=Atp5i PE=
$.688 \mid>$ sp $\mid$ Q9WVH9|FBLN5_MOUSE Fibulin-5 OS=Mus musculus GN=Fbln5 PE=2 SV=1

.690 >sp|Q05186|RCN1_MOUSE Reticulocalbin-1 OS=Mus musculus GN=Rcn1 PE=1 SV=1 $0.693>$ >sp|P19221|THRB_MOUSE Prothrombin OS=Mus musculus GN=F2 PE=1 SV=1

$.695>>$ pp|P07091|S10A4_MOUSE Protein S100-A4 OS=Mus musculus GN=S100a4 PE=1 SV=1 $>$ sp|P70302|STIM1_MOUSE Stromal interaction molecule 1 OS=Mus musculus GN=Stim1 P > >sp|Q9EQP2|EHD4_MOUSE EH domain-containing protein 4 OS=Mus musculus GN=Ehd4 >sp|O35887|CALU_MOUSE Calumenin OS=Mus musculus GN=Calu PE=1 SV=1 $>$ PD|Q9ESD7|DYSF MOUSE Dysferlin OS=Mus musculus GN=Dysf $\mathrm{PE}=1 \mathrm{SV}=3$ $>$ sp|Q60902|EP15R_MOUSE Epidermal growth factor receptor substrate 15-like 1 OS=Mus >sp|Q9D8Y0|EFHD2_MOUSE EF-hand domain-containing protein D2 OS=Mus musculus G $>$ sp|P12815|PDCD6_MOUSE Programmed cell death protein 6 OS=Mus musculus GN=Pdcd $>$ sp|P97347|RPTN_MOUSE Repetin OS=Mus musculus GN=Rptn PE=1 SV=1

$>$ sp|Q9WVJ9|FBLN4_MOUSE EGF-containing fibulin-like extracellular matrix protein 2 OS= $>$ sp|Q06138|CAB39_MOUSE Calcium-binding protein 39 OS=Mus musculus GN=Cab39 PE= $>$ sp|Q9WTR5|CAD13_MOUSE Cadherin-13 OS=Mus musculus GN=Cdh13 PE=1 SV=2 sp|Q9JI91|ACTN2_MOUSE Alpha-actinin-2 OS=Mus musculus GN=Actn2 PE=1 SV=2 $>$ sp|Q61233|PLSL_MOUSE Plastin-2 OS=Mus musculus GN=Lcp1 PE=1 SV=4 >sp|Q8R3B1|PLCD1_MOUSE 1-phosphatidylinositol 4,5-bisphosphate phosphodiesterase del (4) >tr|A2RS43|A2RS43_MOUSE Protein Pcdh7 OS=Mus musculus GN=Pcdh7 PE=2 SV=1 >sp|Q8BLY1|SMOC1_MOUSE SPARC-related modular calcium-binding protein 1 OS=Mus >sp|P43025|TETN_MOUSE Tetranectin OS=Mus musculus GN=Clec3b PE=1 SV=2 $>$ sp|Q8BP92|RCN2_MOUSE Reticulocalbin-2 OS=Mus musculus GN=Rcn2 PE=2 SV=1 4 >sp|O88456|CPNS1_MOUSE Calpain small subunit 1 OS=Mus musculus GN=Capns1 PE=2 >tr|A3KGU7|A3KGU7_MOUSE Protein Spna2 OS=Mus musculus GN=Spna2 PE=4 SV=1 $99>$ >sp|O35639|ANXA3_MOUSE Annexin A3 OS=Mus musculus GN=Anxa3 PE $=1 \mathrm{SV}=4$ $>$ sp|Q61554|FBN1_MOUSE Fibrillin-1 OS=Mus musculus GN=Fbn1 PE=1 SV=1

>tr|A1BN54|A1BN54_MOUSE Alpha actinin 1a OS=Mus musculus GN=Actn1 PE=2 SV=1 >sp|Q8R429|AT2A1_MOUSE Sarcoplasmic/endoplasmic reticulum calcium ATPase 1 OS=M >sp|P52430|PON1_MOUSE Serum paraoxonase/arylesterase 1 OS=Mus musculus GN=Pon1 $>$ sp|P52633|STAT6_MOUSE Signal transducer and transcription activator 6 OS=Mus muscul 4 >sp|P07356|ANXA2_MOUSE Annexin A2 OS=Mus musculus GN=Anxa2 PE=1 SV=2 7 >tr|Q91UZ1|Q91UZ1_MOUSE Phospholipase C beta 4 OS=Mus musculus GN=Plcb4 PE=2 S $>$ sp|P35441|TSP1_MOUSE Thrombospondin-1 OS=Mus musculus GN=Thbs1 PE=1 SV=1

$0.781>$ sp|Q62165|DAG1_MOUSE Dystroglycan OS=Mus musculus GN=Dag1 PE=1 SV=4 0.784 >sp|P97384|ANX11_MOUSE Annexin A11 OS=Mus musculus GN=Anxa11 PE=1 SV=2 0.786 >sp|P63028|TCTP_MOUSE Translationally-controlled tumor protein OS=Mus musclus GN= 0.789 >sp|Q8BH59|CMC1_MOUSE Calcium-binding mitochondrial carrier protein Aralar1 OS=Mu $0.791>$ sp|Q91ZX7|LRP1_MOUSE Prolow-density lipoprotein receptor-related protein 1 OS=Mus m $0.794>\operatorname{tr}|\mathrm{Q} 6 X L Q 8| Q 6 X L Q 8$ MOUSE Calumenin OS=Mus musculus GN=Calu PE=2 SV=1 $0.796>>s p \mid$ O70423|AOC3_MOUSE Membrane primary amine oxidase OS=Mus musculus GN=Aoc 3 0.799 >sp|O35350|CAN1_MOUSE Calpain-1 catalytic subunit OS=Mus musculus GN=Capn1 PE=2 $0.801>>s p \mid$ P08207|S10AA_MOUSE Protein S100-A10 OS=Mus musculus GN=S100a10 PE=2 SV= 0.804 >sp|Q9WVK4|EHD1_MOUSE EH domain-containing protein $1 \mathrm{OS}=$ Mus musculus GN=Ehd $0.806 \mid>$ sp $\mid$ P61022|CHP1_MOUSE Calcineurin B homologous protein $1 \mathrm{OS}=$ Mus musculus GN=Chp 
$>$ sp|P14152|MDHC_MOUSE Malate dehydrogenase, cytoplasmic OS=Mus musculus GN=Mdh1 PE=1 sp|Q01768|NDKB_MOUSE Nucleoside diphosphate kinase B OS=Mus musculus GN=Nme2 PE=1 SV $>$ sp|Q9CXT8|MPPB_MOUSE Mitochondrial-processing peptidase subunit beta OS=Mus musculus GN $>$ sp|P16675|PPGB_MOUSE Lysosomal protective protein OS=Mus musculus GN=Ctsa PE=1 SV=1 $>$ sp|P12787|COX5A_MOUSE Cytochrome c oxidase subunit 5A, mitochondrial OS=Mus musculus GN $>$ sp|Q8K2B3|DHSA_MOUSE Succinate dehydrogenase [ubiquinone] flavoprotein subunit, mitochondri $>$ sp|Q99LC3|NDUAA_MOUSE NADH dehydrogenase [ubiquinone] 1 alpha subcomplex subunit $10, \mathrm{~m}$ $>$ sp|Q9DCS3|MECR_MOUSE Trans-2-enoyl-CoA reductase, mitochondrial OS=Mus musculus GN=M sp|Q60715|P4HA1_MOUSE Prolyl 4-hydroxylase subunit alpha-1 OS=Mus musculus GN=P4ha1 PE= sp|Q9DC69|NDUA9_MOUSE NADH dehydrogenase [ubiquinone] 1 alpha subcomplex subunit 9, mit sp|Q9CR62|M2OM_MOUSE Mitochondrial 2-oxoglutarate/malate carrier protein OS=Mus musculus ( $>$ sp|Q9WTQ8|TIM23 MOUSE Mitochondrial import inner membrane translocase subunit Tim23 OS= SPIQ91YT0|NDUV $>$ sp|P19536|COX5B_MOUSE Cytochrome c oxidase subunit 5B mitochondrial OS=Mus musculus GN $>$ sp|P54310|LIPS_MOUSE Hormone-sensitive lipase OS=Mus musculus GN=Lipe PE=1 SV=2 sp|Q7TMF3|NDUAC_MOUSE NADH dehydrogenase [ubiquinone] 1 alpha subcomplex subunit $12 \mathrm{O}$ sp|Q60575-3|KIF1B_MOUSE Isoform 3 of Kinesin-like protein KIF1B OS=Mus musculus GN=Kif1b sp|P56392|CX7A1_MOUSE Cytochrome c oxidase subunit 7A1, mitochondrial OS=Mus musculus GI $>$ sp|Q60931|VDAC3_MOUSE Voltage-dependent anion-selective channel protein 3 OS=Mus musculus $>$ sp|P17710|HXK1_MOUSE Hexokinase-1 OS=Mus musculus GN=Hk1 PE=1 SV=3

$>$ sp|P18572|BASI_MOUSE Basigin OS=Mus musculus GN=Bsg PE=1 SV=2

$>$ sp|Q9DCJ5|NDUA8_MOUSE NADH dehydrogenase [ubiquinone] 1 alpha subcomplex subunit 8 OS= $>$ sp|Q9DCJ5|NDUA8_MOUSE NADH dehydrogenase [ubiquinone] 1 alpha subcomplex subunit 8 OS= sp|P00397|COX1_MOUSE Cytochrome c oxidase subunit 1 OS=Mus musculus GN=Mtco1 PE=3 SV sp|Q99KK9|SYHM_MOUSE Probable histidine--tRNA ligase, mitochondrial OS=Mus musculus GN= sp|Q9CR68|UCRI_MOUSE Cytochrome b-c1 complex subunit Rieske, mitochondrial OS=Mus muscu $>$ sp|P99028|QCR6_MOUSE Cytochrome b-c1 complex subunit 6, mitochondrial OS=Mus musculus GN $>$ sp|A6H611|MIPEP_MOUSE Mitochondrial intermediate peptidase OS=Mus musculus GN=Mipep PE $>$ sp|Q9Z110|P5CS_MOUSE Delta-1-pyrroline-5-carboxylate synthase OS=Mus musculus GN=Aldh18a sp|P31938|MP2K1_MOUSE Dual specificity mitogen-activated protein kinase kinase 1 OS=Mus musc sp|Q9CPQ3|TOM22_MOUSE Mitochondrial import receptor subunit TOM22 homolog OS=Mus musc $>$ sp $\mid$ Q8BH59|CMC1_MOUSE Calcium-binding mitochondrial carrier protein Aralar1 OS=Mus musculu $>$ sp|Q62167|DDX3X_MOUSE ATP-dependent RNA helicase DDX3X OS=Mus musculus GN=Ddx3x $>$ sp|Q99L04|DHRS1_MOUSE Dehydrogenase/reductase SDR family member 1 OS=Mus musculus GN $>$ sp|P31786|ACBP_MOUSE Acyl-CoA-binding protein OS=Mus musculus GN=Dbi PE=1 SV=2 $>$ sp|Q91V61|SFXN3_MOUSE Sideroflexin-3 OS=Mus musculus GN=Sfxn3 PE=1 SV=1 sp|Q9CPP6|NDUA5_MOUSE NADH dehydrogenase [ubiquinone] 1 alpha subcomplex subunit 5 OS= $>$ sp|Q64442|DHSO_MOUSE Sorbitol dehydrogenase OS=Mus musculus GN=Sord PE=1 SV=3 sp|Q8BGC4|ZADH2_MOUSE Zinc-binding alcohol dehydrogenase domain-containing protein 2 OS= $>$ sp|Q8BGH2|SAM50_MOUSE Sorting and assembly machinery component 50 homolog OS=Mus mus $>$ sp|Q9CPQ1|COX6C_MOUSE Cytochrome c oxidase subunit 6C OS=Mus musculus GN=Cox6c PE=1 tr|Q91WX5|Q91WX5_MOUSE Bak1 protein OS=Mus musculus GN=Bak1 PE=2 SV=1

tr|Q8R5L1|Q8R5L1_MOUSE Complement component $1 \mathrm{Q}$ subcomponent-binding protein, mitochond $>$ sp|P54822|PUR8_MOUSE Adenylosuccinate lyase OS=Mus musculus GN=Adsl PE=2 SV=2

sp|Q9CZ13|QCR1_MOUSE Cytochrome b-c1 complex subunit 1, mitochondrial OS=Mus musculus $C$ $>$ sp|Q9ERS2|NDUAD_MOUSE NADH dehydrogenase [ubiquinone] 1 alpha subcomplex subunit 13 Os $>$ sp|P67778|PHB MOUSE Prohibitin OS=Mus musculus GN=Phb PE=1 SV=1

sp|Q9CQ75|NDUA2_MOUSE NADH dehydrogenase [ubiquinone] 1 alpha subcomplex subunit 2 OS=

$>$ sp|Q922Q1|MOSC2_MOUSE MOSC domain-containing protein 2, mitochondrial OS=Mus musculus (
.809|>sp|P14824|ANXA6_MOUSE Annexin A6 OS=Mus musculus GN=Anxa6 PE=1 SV=3

$0.811>$ >sp|O70258|SGCE_MOUSE Epsilon-sarcoglycan OS=Mus musculus GN=Sgce PE=2 SV=2 0.814 >sp|P14211|CALR_MOUSE Calreticulin OS=Mus musculus GN=Calr PE=1 SV=1

0.816 >sp|Q8BH97|RCN3_MOUSE Reticulocalbin-3 OS=Mus musculus GN=Rcn3 PE=2 SV=1 |P10493|NID1_MOUSE Nidogen-1 OS=Mus musculus GN=Nid1 PE=1 SV=2

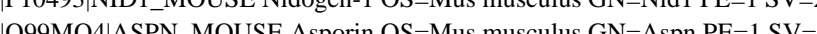
|Q61576|FKB10_MOUSE Peptidyl-prolyl cis-trans isomerase FKBP10 OS=Mus musculus |Q9QXY6|EHD3_MOUSE EH domain-containing protein 3 OS=Mus musculus GN=Ehd3 p|P62748|HPCL1_MOUSE Hippocalcin-like protein 1 OS=Mus musculus GN=Hpcal1 PE=: P50543|S10AB_MOUSE Protein S100-A11 OS=Mus musculus GN=S100a11 PE=2 SV=1 P47713|PA24A_MOUSE Cytosolic phospholipase A2 OS=Mus musculus GN=Pla2g4a PI |Q9Z247|FKBP9_MOUSE Peptidyl-prolyl cis-trans isomerase FKBP9 OS=Mus musculus Sp|P42225|STAT1_MOUSE Signal transducer and activator of transcription 1 OS=Mus mus |Q02819|NUCB1_MOUSE Nucleobindin-1 OS=Mus musculus GN=Nucb1 PE=1 SV=2 P82350|SGCA_MOUSE Alpha-sarcoglycan OS=Mus musculus GN=Sgca PE=1 SV=1 作 sp|Q3UYK3|TBCD9_MOUSE TBC1 domain family member 9 OS=Mus musculus GN=Tbc Sp|Q99M71|EPDR1_MOUSE Mammalian ependymin-related protein 1 OS=Mus musculus $>$ pp|P35564|CALX_MOUSE Calnexin OS=Mus musculus GN=Canx PE=1 SV=1 sp|Q64521|GPDM_MOUSE Glycerol-3-phosphate dehydrogenase, mitochondrial OS=Mus n 作

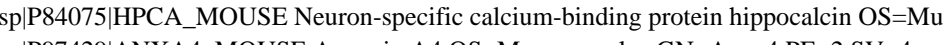

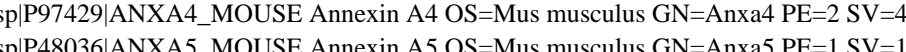
sp|Q9Z184|PADI3_MOUSE Protein-arginine deiminase type-3 OS=Mus musculus GN=Pad

\section{Lipid metabolic process GO:0006629}

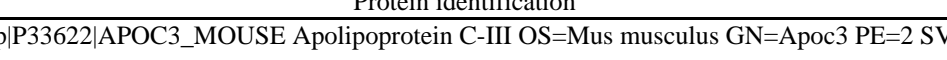
Q00623|APOA1_MOUSE Apolipoprotein A-I OS $=$ Mus musculus GN=Apoa1 PE $=1$
P01027|CO3_MOUSE Complement C3 OS=Mus musculus GN=C3 PE $=1$ SV $=3$ p|Q9Z2V4|PCKGC_MOUSE Phosphoenolpyruvate carboxykinase, cytosolic [GTP] OS=M 35452|O35452_MOUSE Protein Tnxb OS=Mus musculus GN=Tnxb PE=2 SV=1 P08226|APOE_MOUSE Apolipoprotein E OS=Mus musculus GN=Apoe PE=1 SV=2 Q (10) O35074|PTGIS_MOUSE Prostacyclin synthase OS=Mus musculus GN=Ptgis $\mathrm{PE}=2 \mathrm{SV}$ Q921H8|THIKA MOUSE 3-ketoacyl-CoA thiolase A, peroxisomal OS=Mus musculus Q9DCN2|NB5R3_MOUSE NADH-cytochrome b5 reductase 3 OS=Mus musculus GN=C |O70503|DHB12_MOUSE Estradiol 17-beta-dehydrogenase 12 OS=Mus musculus GN=H |Q8VCW8|ACSF2_MOUSE Acyl-CoA synthetase family member 2, mitochondrial OS=M p|P22437|PGH1_MOUSE Prostaglandin G/H synthase 1 OS=Mus musculus GN=Ptgs1 PE= P|Q9R0Q7|TEBP_MOUSE Prostaglandin E synthase 3 OS=Mus musculus GN=Ptges3 PE= |Q8R3B1|PLCD1_MOUSE 1-phosphatidylinositol 4,5-bisphosphate phosphodiesterase del P|Q9JHU9|INO1_MOUSE Inositol-3-phosphate synthase 1 OS=Mus musculus GN=Isyna1 ] 0.925 >sp|P51174|ACADL_MOUSE Long-chain specific acyl-CoA dehydrogenase, mitochondrial O
0.927 >sp|Q9DBL1|ACDSB_MOUSE Short/branched chain specific acyl-CoA dehydrogenase, mitor 
>sp|Q9R0X4|ACOT9_MOUSE Acyl-coenzyme A thioesterase 9, mitochondrial OS=Mus musculus GN $>$ sp|P48962|ADT1_MOUSE ADP/ATP translocase 1 OS=Mus musculus GN=Slc25a4 PE=1 SV=4 $>$ sp|Q9D6J6|NDUV2_MOUSE NADH dehydrogenase [ubiquinone] flavoprotein 2, mitochondrial OS= >sp|P56391|CX6B1_MOUSE Cytochrome c oxidase subunit 6B1 OS=Mus musculus GN=Cox6b1 PE=1 $>$ sp|Q9CQA3|DHSB_MOUSE Succinate dehydrogenase [ubiquinone] iron-sulfur subunit, mitochondrial $>$ sp|P30416|FKBP4_MOUSE Peptidyl-prolyl cis-trans isomerase FKBP4 OS=Mus musculus GN=Fkbp $>$ sp|Q8CAQ8|IMMT_MOUSE Mitochondrial inner membrane protein OS=Mus musculus GN=Immt PE $>$ sp|P13707|GPDA_MOUSE Glycerol-3-phosphate dehydrogenase [NAD(+)], cytoplasmic OS=Mus mu $>$ sp|Q9DB77|QCR2_MOUSE Cytochrome b-c1 complex subunit 2, mitochondrial OS=Mus musculus $\mathrm{C}$ $>$ sp|O35129|PHB2_MOUSE Prohibitin-2 OS=Mus musculus GN=Phb2 PE=1 SV=1

$>$ sp|P51881|ADT2_MOUSE ADP/ATP translocase 2 OS=Mus musculus GN=Slc25a5 PE=1 SV=3

$>$ sp|Q9CQ69|QCR8_MOUSE Cytochrome b-c1 complex subunit 8 OS=Mus musculus GN=Uqcrq PE=1 $>$ sp|Q8K3J1|NDUS8 MOUSE NADH dehydrogenase [ubiquinone] iron-sulfur protein 8, mitochondrial $>$ sp|P03888|NU1M_MOUSE NADH-ubiquinone oxidoreductase chain 1 OS=Mus musculus GN=Mtnd $>$ sp|Q9DCS9|NDUBA_MOUSE NADH dehydrogenase [ubiquinone] 1 beta subcomplex subunit $10 \mathrm{OS}=$ >sp|Q9DB20|ATPO_MOUSE ATP synthase subunit $\mathrm{O}$, mitochondrial OS=Mus musculus GN=Atp5o P $>$ sp|Q9R269|PEPL_MOUSE Periplakin OS=Mus musculus GN=Ppl PE=1 SV=1

sp|P20108|PRDX3 MOUSE Thioredoxin-dependent peroxide reductase, mitochondrial OS=Mus musc $>$ sp|O08807|PRDX4_MOUSE Peroxiredoxin-4 OS=Mus musculus GN=Prdx4 PE=1 SV=1

$>$ sp|P19783|COX41_MOUSE Cytochrome c oxidase subunit 4 isoform 1, mitochondrial OS=Mus musc $>$ sp|Q8R1I1|QCR9_MOUSE Cytochrome b-c1 complex subunit 9 OS=Mus musculus GN=Uqcr10 PE= $>$ sp|Q9CPQ8|ATP5L_MOUSE ATP synthase subunit g, mitochondrial OS=Mus musculus GN=Atp5l P $>$ sp|Q91W97|HKDC1_MOUSE Putative hexokinase HKDC1 OS=Mus musculus GN=Hkdc1 PE=2 SV $>$ sp|Q3UIU2|NDUB6_MOUSE NADH dehydrogenase [ubiquinone] 1 beta subcomplex subunit 6 OS=N sp|Q9D0M3|CY1_MOUSE Cytochrome c1, heme protein, mitochondrial OS=Mus musculus GN=Cyc $>$ sp|Q9CQQ7|AT5F1_MOUSE ATP synthase subunit b, mitochondrial OS=Mus musculus GN=Atp5f1 $>$ sp|Q99LY9|NDUS5_MOUSE NADH dehydrogenase [ubiquinone] iron-sulfur protein 5 OS=Mus mus sp|P12242|UCP1_MOUSE Mitochondrial brown fat uncoupling protein 1 OS=Mus musculus GN=Ucp $>$ sp|Q8BQU3|ACN9_MOUSE Protein ACN9 homolog, mitochondrial OS=Mus musculus GN=Acn9 PE

Extracellular matrix GO:0031012

\begin{tabular}{|c|c|c|}
\hline Protein identification & $\mathrm{Zq}$ & $\mathrm{rank} / \mathrm{N}$ \\
\hline |Q05504|Q05504_MOUSE Collagen alpha 3 chain type VI (Fragment) OS=Mus musculus GN=Col6a & -4.119 & 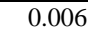 \\
\hline |Q9Z1T2|TSP4_MOUSE Thrombospondin-4 OS=Mu & -2.244 & \\
\hline & -2.195 & \\
\hline 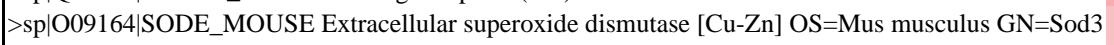 & -1.698 & 0.039 \\
\hline sp|P58459|ATS1 & -1.626 & 0.051 \\
\hline sp|P12246|SAM & -1.551 & 0.062 \\
\hline $\mathrm{sp} \mid \mathrm{Q} \mathrm{s}$ & -1.312 & 0.073 \\
\hline $\mathrm{sp} \mid \mathrm{Q}$ & -1.257 & 0.08 \\
\hline$>\operatorname{tr} \mid \mathrm{F} 8 \mathrm{Y}$ & -1.144 & \\
\hline 资 & -1.139 & \\
\hline $\mathrm{tr} \mid \mathrm{Q} 8$ & -1.120 & 0.1 \\
\hline$>$ sp|P28481|c & -0.986 & 0.12 \\
\hline SE Nidogen- $1 \mathrm{OS}=$ Mus musculus GN=Nid1 $\mathrm{PE}=1 \mathrm{SV}=2$ & -0.927 & \\
\hline sp|Q9DC & -0.801 & 0.152 \\
\hline & -0.686 & 0.16 \\
\hline & 0.676 & \\
\hline 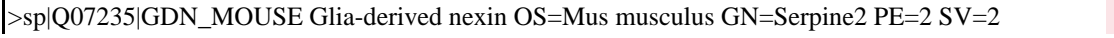 & 655 & 0.1 \\
\hline
\end{tabular}
sp|P42125|ECI1_MOUSE Enoyl-CoA delta isomerase 1, mitochondrial OS=Mus musculus sp|P35235|PTN11_MOUSE Tyrosine-protein phosphatase non-receptor type 11 OS=Mus mu $>$ sp|P47713|PA24A_MOUSE Cytosolic phospholipase A2 OS=Mus musculus GN=Pla2g4a PI sp|Q8BMP6|GCP60_MOUSE Golgi resident protein GCP60 OS=Mus musculus GN=Acbd3 sp|Q9D2R0|AACS_MOUSE Acetoacetyl-CoA synthetase OS=Mus musculus GN=Aacs PE= sp|Q03265|ATPA_MOUSE ATP synthase subunit alpha, mitochondrial OS=Mus musculus sp|Q8VDP6|CDIPT_MOUSE CDP-diacylglycerol--inositol 3-phosphatidyltransferase OS=M sp|O08709|PRDX6_MOUSE Peroxiredoxin-6 OS=Mus musculus GN=Prdx6 PE=1 SV=3 sp|Q05816|FABP5_MOUSE Fatty acid-binding protein, epidermal OS=Mus musculus GN=F sp|O88307|SORL_MOUSE Sortilin-related receptor OS=Mus musculus GN=Sorl1 PE=2 SV $>$ sp|Q61206|PA1B2_MOUSE Platelet-activating factor acetylhydrolase IB subunit beta OS=M sp|Q3TCN2|PLBL2_MOUSE Putative phospholipase B-like 2 OS=Mus musculus GN=Plbd2 sp|Q8VCT4|CES1D_MOUSE Carboxylesterase 1D OS=Mus musculus GN=Ces1d PE=1 SV sp|P50171|DHB8_MOUSE Estradiol 17-beta-dehydrogenase 8 OS=Mus musculus GN=Hsd1 $>$ sp|P52840|ST1A1_MOUSE Sulfotransferase 1A1 OS=Mus musculus GN=Sult1a1 PE=2 SV: 
sp|Q61398|PCOC1_MOUSE Procollagen C-endopeptidase enhancer 1 OS=Mus musculus GN=Pcolce $>$ sp|P07901|HS90A_MOUSE Heat shock protein HSP 90-alpha OS=Mus musculus GN=Hsp90aa1 PE= $>$ sp|Q9D2L5|CPXM2_MOUSE Inactive carboxypeptidase-like protein X2 OS=Mus musculus GN=Cpxı $>$ sp|Q8CIZ8|VWF_MOUSE von Willebrand factor OS=Mus musculus GN=Vwf PE=1 SV=2

$>\operatorname{tr}|\mathrm{Q} 3 T A F 9| \mathrm{Q} 3 T$ AF9_MOUSE Putative uncharacterized protein OS=Mus musculus $\mathrm{GN}=\mathrm{Bgn} \mathrm{PE}=2 \mathrm{SV}$ $>$ sp|Q99K41|EMIL1_MOUSE EMILIN-1 OS=Mus musculus GN=Emilin1 PE=1 SV=1

$>$ sp|Q640N1|AEBP1_MOUSE Adipocyte enhancer-binding protein 1 OS=Mus musculus GN=Aebp1 PI $>$ sp $\mid$ P11087|CO1A1_MOUSE Collagen alpha-1(I) chain OS=Mus musculus GN=Col1a1 PE=1 SV=4 sp $|\mathrm{Q} 00780|$ CO8A1_MOUSE Collagen alpha-1(VIII) chain OS=Mus musculus GN=Col8a1 PE=1 SV= tr|Q99K31|Q99K31_MOUSE Col6a3 protein (Fragment) OS=Mus musculus GN=Col6a3 PE=2 SV=1 $>$ sp|P08121|CO3A1_MOUSE Collagen alpha-1(III) chain OS=Mus musculus GN=Col3a1 PE=2 SV=4 $>$ sp|Q80YX1|TENA_MOUSE Tenascin OS=Mus musculus GN=Tnc PE $=1 \mathrm{SV}=1$

$>$ sp|O88322|NID2 MOUSE Nidogen-2 OS=Mus musculus GN=Nid2 PE=1 SV=2

$>$ sp|P28653|PGS1_MOUSE Biglycan OS=Mus musculus GN=Bgn PE=2 SV=1

$>$ sp|Q61554|FBN1_MOUSE Fibrillin-1 OS=Mus musculus GN=Fbn1 PE=1 SV=1

$>$ sp|Q3UQ28|PXDN_MOUSE Peroxidasin homolog OS=Mus musculus GN=Pxdn PE=2 SV=2

sp|Q8CD91|SMOC2_MOUSE SPARC-related modular calcium-binding protein 2 OS=Mus musculus

sp|Q8CG19|LTBP1_MOUSE Latent-transforming growth factor beta-binding protein 1 OS=Mus musc $>$ sp $\mid$ Q01149|CO1A2_MOUSE Collagen alpha-2(I) chain OS=Mus musculus GN=Col1a2 PE=2 SV=2 $>\operatorname{tr}|\mathrm{O} 88493| \mathrm{O} 88493$ MOUSE Type VI collagen alpha 3 subunit OS=Mus musculus GN=Col6a3 PE=2 $>$ sp|P97873|LOXL1_MOUSE Lysyl oxidase homolog 1 OS=Mus musculus GN=Loxl1 PE=2 SV=3

tr|Q9Z0I9|Q9Z0I9_MOUSE Collagen alpha3(VI) (Fragment) OS=Mus musculus GN=Col6a3 PE=2 S sp|P08228|SODC_MOUSE Superoxide dismutase [Cu-Zn] OS=Mus musculus GN=Sod1 PE=1 SV=2 sp|Q8R2G6|CCD80_MOUSE Coiled-coil domain-containing protein 80 OS=Mus musculus GN=Ccdc tr|Q3UZF9|Q3UZF9_MOUSE Putative uncharacterized protein (Fragment) OS=Mus musculus GN=Fn $>$ sp|Q9QZZ6|DERM_MOUSE Dermatopontin OS=Mus musculus GN=Dpt PE=2 SV=1

$>$ sp|Q08879-2|FBLN1_MOUSE Isoform C of Fibulin-1 OS=Mus musculus GN=Fbln1

$>\operatorname{tr|} \mid$ B1B0C7|B1B0C7_MOUSE Basement membrane-specific heparan sulfate proteoglycan core protein $>$ sp|Q62005|ZP1_MOUSE Zona pellucida sperm-binding protein $1 \mathrm{OS}=$ Mus musculus GN=Zp1 PE=1 S sp|Q8C4U3|SFRP1_MOUSE Secreted frizzled-related protein 1 OS=Mus musculus GN=Sfrp1 PE=1 S tr|B7ZNH7|B7ZNH7_MOUSE Collagen alpha-1(XIV) chain OS=Mus musculus GN=Col14a1 PE=2 S $>$ sp|P97298|PEDF_MOUSE Pigment epithelium-derived factor OS=Mus musculus GN=Serpinf1 PE=1 $>$ sp|P27090|TGFB2_MOUSE Transforming growth factor beta-2 OS=Mus musculus GN=Tgfb2 PE=1 $\mathrm{s}$ tr|A2AQ53|A2AQ53_MOUSE Fibrillin $1 \mathrm{OS}=$ Mus musculus $\mathrm{GN}=\mathrm{Fbn} 1 \mathrm{PE}=4 \mathrm{SV}=1$

$>$ sp|P28654|PGS2_MOUSE Decorin OS=Mus musculus GN=Dcn PE=2 SV=1

$>$ sp|Q61001|LAMA5_MOUSE Laminin subunit alpha-5 OS=Mus musculus GN=Lama5 PE=1 SV=4 tr $\mid$ Q3UHH3|Q3UHH3_MOUSE Putative uncharacterized protein OS=Mus musculus GN=Hspg2 PE=2 sp|P82198|BGH3_MOUSE Transforming growth factor-beta-induced protein ig-h3 OS=Mus musculus $>$ sp|P21844|CMA1_MOUSE Chymase OS=Mus musculus GN=Cma1 PE=1 SV=2

sp|Q62219|TGFI1_MOUSE Transforming growth factor beta-1-induced transcript 1 protein OS=Mus r $>$ sp|P50608|FMOD_MOUSE Fibromodulin OS=Mus musculus GN=Fmod PE=2 SV=1

$>$ sp|P33434|MMP2_MOUSE $72 \mathrm{kDa}$ type IV collagenase OS=Mus musculus GN=Mmp2 PE=2 SV=1

$>$ sp|Q62009-2|POSTN_MOUSE Isoform 2 of Periostin OS=Mus musculus GN=Postn

$>$ sp|Q61292|LAMB2_MOUSE Laminin subunit beta-2 OS=Mus musculus GN=Lamb2 PE=2 SV=2

$>$ sp $\mid$ P18242|CATD_MOUSE Cathepsin D OS=Mus musculus GN=Ctsd PE $=1 \mathrm{SV}=1$

sp|P14211|CALR_MOUSE Calreticulin OS=Mus musculus GN=Calr PE=1 SV=1

$>$ sp|Q04857|CO6A1_MOUSE Collagen alpha-1(VI) chain OS=Mus musculus GN=Col6a1 PE=2 SV=1 $>$ sp|Q06890|CLUS_MOUSE Clusterin OS=Mus musculus GN=Clu PE $=1 \mathrm{SV}=1$

$>$ sp|Q02788|CO6A2_MOUSE Collagen alpha-2(VI) chain OS=Mus musculus GN=Col6a2 PE=2 SV=3
$-0.618$

- $0.208>\mathrm{sp}|\mathrm{O} 35678|$ MGLL_MOUSE Monoglyceride lipase OS=Mus musculus GN=Mgll PE=1 SV=1 >sp|Q8JZK9|HMCS1_MOUSE Hydroxymethylglutaryl-CoA synthase, cytoplasmic OS=Mus $0.230>$ >sp|Q9DBL9|ABHD5_MOUSE 1-acylglycerol-3-phosphate O-acyltransferase ABHD5 OS=M

$-0.500 \quad 0.242>$ sp|Q9CY27|TECR_MOUSE Very-long-chain enoyl-CoA reductase OS=Mus musculus GN= $-0.333$ $-0.328 \quad 0.287$ $\begin{array}{ll}-0.324 & 0.298\end{array}$ $-0.263 \quad 0.309$ $-0.156 \quad 0.320$ $-0.137 \quad 0.33$

$-0.137 \quad 0.343$

$-0.136 \quad 0.354$ $-0.134 \quad 0.365$ $-0.074 \quad 0.376$ $\begin{array}{cc}-0.012 & 0.38 \\ 0.035 & 0.399\end{array}$ $\begin{array}{ll}0.035 & 0.399\end{array}$ sp|Q9EQ06|DHB11_MOUSE Estradiol 17-beta-dehydrogenase 11 OS=Mus musculus GN= sp|Q8BMS1|ECHA_MOUSE Trifunctional enzyme subunit alpha, mitochondrial OS=M $>$ sp|P51910|APOD_MOUSE Apolipoprotein D OS=Mus musculus GN=Apod PE=2 SV=1 sp|P50172|DHI1_MOUSE Corticosteroid 11-beta-dehydrogenase isozyme 1 OS=Mus muscu
sp|Q07417|ACADS_MOUSE Short-chain specific acyl-CoA dehydrogenase, mitochondrial sp|Q8CGN5|PLIN1_MOUSE Perilipin-1 OS=Mus musculus GN=Plin1 PE=1 SV=2

\section{Mitochondrion GO:0005739}

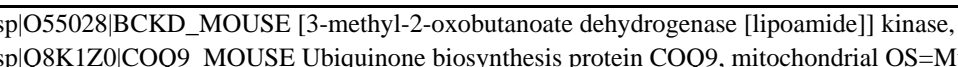

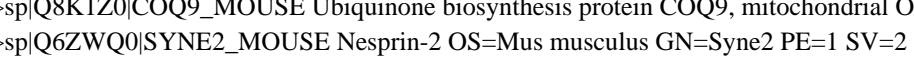
sp|Q06890|CLUS_MOUSE Clusterin OS=Mus musculus GN=Clu PE=1 SV=1

sp|Q8BGC4|ZADH2_MOUSE Zinc-binding alcohol dehydrogenase domain-containing prote $>$ sp|O09164|SODE_MOUSE Extracellular superoxide dismutase [Cu-Zn] OS=Mus musculus $>$ sp|Q920A7|AFG31_MOUSE AFG3-like protein 1 OS=Mus musculus GN=Afg311 PE=2 SV sp|Q8BP47|SYNC_MOUSE Asparagine--tRNA ligase, cytoplasmic OS=Mus musculus GN sp|Q60931|VDAC3_MOUSE Voltage-dependent anion-selective channel protein 3 OS=Mu tr|Q8R5L1|Q8R5L1_MOUSE Complement component 1 Q subcomponent-binding protein, sp|Q91V61|SFXN3_MOUSE Sideroflexin-3 OS=Mus musculus GN=Sfxn3 PE=1 SV=1 sp|Q61171|PRDX2_MOUSE Peroxiredoxin-2 OS=Mus musculus GN=Prdx2 PE=1 SV=3 sp|Q9Z2Y8|PROSC_MOUSE Proline synthase co-transcribed bacterial homolog protein OS sp|Q9DC29|ABCB6_MOUSE ATP-binding cassette sub-family B member 6, mitochondrial sp|Q9JM14|NT5C_MOUSE 5'(3')-deoxyribonucleotidase, cytosolic type OS=Mus musculus $>$ sp|Q8CH40|NUDT6_MOUSE Nucleoside diphosphate-linked moiety X motif 6 OS=Mus m sp|Q3THF9|CQ10B_MOUSE Coenzyme Q-binding protein COQ10 homolog B, mitochondri $>$ sp|Q8VCF0|MAVS_MOUSE Mitochondrial antiviral-signaling protein OS=Mus musculus $\mathrm{C}$ sp|O35658|C1QBP_MOUSE Complement component $1 \mathrm{Q}$ subcomponent-binding protein, $\mathrm{m}$ $>$ tr|Q3U2G2|Q3U2G2_MOUSE Heat shock $70 \mathrm{kDa}$ protein $4 \mathrm{OS}=$ Mus musculus GN=Hspa4 sp|P13707|GPDA_MOUSE Glycerol-3-phosphate dehydrogenase [NAD(+)], cytoplasmic OS tr|D3Z7C8|D3Z7C8_MOUSE Branched-chain-amino-acid aminotransferase OS=Mus muscu $>$ sp|P21981|TGM2_MOUSE Protein-glutamine gamma-glutamyltransferase 2 OS=Mus musc sp|Q9DCS9|NDUBA_MOUSE NADH dehydrogenase [ubiquinone] 1 beta subcomplex subu sp|Q8BGB8|COQ4_MOUSE Ubiquinone biosynthesis protein COQ4 homolog, mitochondri sp|O08756|HCD2_MOUSE 3-hydroxyacyl-CoA dehydrogenase type-2 OS=Mus musculus IB1AWZ3|B1AWZ3_MOUSE Novel prote sp|Q99JR1|SFXN1_MOUSE Sideroflexin-1 OS=Mus musculus GN=Sfxn1 PE=1 SV=3 sp|P56395|CYB5_MOUSE Cytochrome b5 OS=Mus musculus GN=Cyb5a PE=1 SV=2 0.680 >sp|P23927|CRYAB_MOUSE Alpha-crystallin B chain OS=Mus musculus GN=Cryab PE=1 $0.691>$ >sp|O70325|GPX41_MOUSE Phospholipid hydroperoxide glutathione peroxidase, mitochond 0.702 >sp|Q9CZW5|TOM70_MOUSE Mitochondrial import receptor subunit TOM70 OS=Mus mus 0.713 >sp|Q9WTP7|KAD3 MOUSE GTP·AMP phosphotransferase, mitochondrial OS=Mus muscu 0.713 >sp|Q9WTP7|KAD3_MOUSE GTP:AMP phosphotransferase, mitochondrial OS=Mus musc 0.736 >sp |O08807|PRDX4_MOUSE Peroxiredoxin-4 OS=Mus musculus GN=Prdx4 PE=1 SV=1 
>sp|Q8VEE1|LMCD1_MOUSE LIM and cysteine-rich domains protein 1 OS=Mus musculus GN=Lmc $>$ sp|O70503|DHB12_MOUSE Estradiol 17-beta-dehydrogenase 12 OS=Mus musculus GN=Hsd17b12 P $>$ sp|P51885|LUM_MOUSE Lumican OS=Mus musculus GN=Lum PE=1 SV=2

$>$ sp|P07214|SPRC_MOUSE SPARC OS=Mus musculus GN=Sparc PE=1 SV=1

$>$ sp|Q05793|PGBM_MOUSE Basement membrane-specific heparan sulfate proteoglycan core protein O $>$ sp|Q01339|APOH_MOUSE Beta-2-glycoprotein 1 OS=Mus musculus GN=Apoh PE=1 SV=1

$>$ sp $\mid$ P11276|FINC_MOUSE Fibronectin OS=Mus musculus GN=Fn1 PE=1 SV=4

sp|Q8C6K9|CO6A6_MOUSE Collagen alpha-6(VI) chain OS=Mus musculus GN=Col6a6 PE=1 SV=2 >tr|B9EHT6|B9EHT6_MOUSE Fn1 protein OS=Mus musculus GN=Fn1 PE=2 SV=1 $>$ sp|Q9WVH9|FBLN5_MOUSE Fibulin-5 OS=Mus musculus GN=Fbln5 PE $=2 \mathrm{SV}=$ $>$ sp|Q62000|MIME_MOUSE Mimecan OS=Mus musculus GN=Ogn PE=2 SV=1

$>$ sp|Q9R118|HTRA1_MOUSE Serine protease HTRA1 OS=Mus musculus GN=Htra1 PE=1 SV=2 $>$ sp|P16045|LEG1_MOUSE Galectin-1 OS=Mus musculus GN=Lgals1 PE=1 SV=3

$>$ sp|Q8K4G1|LTBP4_MOUSE Latent-transforming growth factor beta-binding protein 4 OS=Mus mus $>$ sp|Q3UTY6|THSD4_MOUSE Thrombospondin type-1 domain-containing protein 4 OS=Mus musculu $>$ sp|Q99JR5|TINAL_MOUSE Tubulointerstitial nephritis antigen-like OS=Mus musculus GN=Tinagl1 $>\mathrm{sp}|\mathrm{P} 08122|$ CO4A2_MOUSE Collagen alpha-2(IV) chain OS=Mus musculus GN=Col4a2 PE $=2 \mathrm{SV}=4$ $>$ sp|P39061|COIA1_MOUSE Collagen alpha-1(XVIII) chain OS=Mus musculus GN=Col18a1 PE=1 SV $>$ sp|Q8BPB5|FBLN3_MOUSE EGF-containing fibulin-like extracellular matrix protein 1 OS=Mus mus $>$ sp|Q61282|PGCA MOUSE Aggrecan core protein OS=Mus musculus GN=Acan PE=1 SV=2

$>$ sp|Q9D1H9|MFAP4_MOUSE Microfibril-associated glycoprotein 4 OS=Mus musculus GN=Mfap4 P $>$ sp $|\mathrm{Q} 61245|$ COBA1_MOUSE Collagen alpha-1(XI) chain OS=Mus musculus GN=Col11a1 PE=1 SV= sp|O35206|COFA1_MOUSE Collagen alpha-1(XV) chain OS=Mus musculus GN=Col15a1 PE=1 SV

\section{Intermediate filament GO:0005882}

$>$ sp|P46660|AINX_MOUSE Alpha-internexin OS=Mus musculus GN=Ina PE=1 SV=2 sp|O55135|IF6_MOUSE Eukaryotic translation initiation factor 6 OS=Mus musculus GN=Eif6 PE=1 $>$ sp|P08551|NFL_MOUSE Neurofilament light polypeptide OS=Mus musculus GN=Nefl PE=1 SV= $>$ sp|P14733|LMNB1_MOUSE Lamin-B1 OS=Mus musculus GN=Lmnb1 PE=1 SV=3 $>$ sp|P15331|PERI_MOUSE Peripherin OS=Mus musculus GN=Prph PE=1 SV=2 $>$ sp|E9Q557|DESP_MOUSE Desmoplakin OS=Mus musculus GN=Dsp PE $=3 \mathrm{SV}=1$ $>$ sp|P48678|LMNA_MOUSE Prelamin-A/C OS=Mus musculus GN=Lmna PE $=1 \mathrm{SV}=2$ $>$ sp|P21619|LMNB2_MOUSE Lamin-B2 OS=Mus musculus GN=Lmnb2 PE=1 SV=2

tr|Q3TFD9|Q3TFD9_MOUSE Putative uncharacterized protein OS=Mus musculus $\mathrm{GN}=\mathrm{Vim} \mathrm{PE}=2$ $>$ sp|P31001|DESM_MOUSE Desmin OS=Mus musculus GN=Des $P E=1$ SV=3 $>$ sp|Q6P5H2|NEST_MOUSE Nestin OS=Mus musculus GN=Nes PE=1 SV=1 sp|P03995|GFAP_MOUSE Glial fibrillary acidic protein OS=Mus musculus GN=Gfap PE=1 SV=4 $>$ sp|Q91ZU6|DYST_MOUSE Dystonin OS=Mus musculus GN=Dst PE $=1$ SV=1

$>$ sp|P20152|VIME MOUSE Vimentin OS=Mus musculus GN=Vim PE=1 SV=3

\section{Muscle contraction GO:0006936}

\begin{tabular}{|c|c|c|}
\hline Protein identification & $\mathrm{Zq}$ & $\mathrm{rank} / \mathrm{N}$ \\
\hline$>$ sp|Q60605|MYL6_MOUSE Myosin light polypeptide 6 OS=Mus musculus GN=Myl6 PE=1 SV=3 & -0.430 & -0.036 \\
\hline |>sp|A2ASS6|TITIN_MOUSE Titin OS=Mus & 0.138 & 0.012 \\
\hline >sp|Q64018|GLRA1_MOUSE Glycine receptor subunit alpha-1 OS=Mus musculus GN=Glra1 PE=1 SI & 0.673 & 0.056 \\
\hline |>sp|P58774-2|TPM2_MOUSE Isoform 2 of Tropomyosin beta chain OS=Mus musculus GN=Tpm2 & 0.737 & 0.061 \\
\hline$>\operatorname{tr}|\mathrm{Q} 8 \mathrm{VCQ} 8| \mathrm{Q} 8 \mathrm{VCQ} 8$ MOUSE Caldesmon $1 \mathrm{OS}=$ Mus musculus GN=Cald1 PE=2 SV=1 & 1.453 & 0.121 \\
\hline tr|F6T2Z7|F6T2Z7_MOUSE Protein Cald1 (Fragment) OS=Mus musculus GN=Cald1 PE & 1.640 & 0.13 \\
\hline
\end{tabular}

>sP|Q97105|CLIC1_MOUSE Chloride intracellular channel protein 1 OS=Mus musculus GN Pp|P08074|CBR2_MOUSE Carbonyl reductase [NADPH] 2 OS=Mus musculus GN=Cbr2 sp|Q8BVI4|DHPR_MOUSE Dihydropteridise [NADPH] 2 OS=Mus musculus GN=Cbr2 sp|Q791V5|MTCH2_MOUSE Mitochondrial carrier homolog 2 OS=Mus musculus GN=Mt $>$ sp|Q8BW75|AOFB_MOUSE Amine oxidase [flavin-containing] B OS=Mus musculus GN= $>$ sp|P14152|MDHC_MOUSE Malate dehydrogenase, cytoplasmic OS=Mus musculus GN=M sp|Q8BU85|MSRB3_MOUSE Methionine-R-sulfoxide reductase B3, mitochondrial OS=M $>$ sp|Q9DBF1|AL7A1_MOUSE Alpha-aminoadipic semialdehyde dehydrogenase OS=Mus mu $>$ sp|Q9D4J1|EFHD1_MOUSE EF-hand domain-containing protein D1 OS=Mus musculus GN sp|Q9DCM2|GSTK1_MOUSE Glutathione S-transferase kappa 1 OS=Mus musculus GN=G $>$ sp|P35700|PRDX1_MOUSE Peroxiredoxin-1 OS=Mus musculus GN=Prdx1 PE=1 SV=1 sp|Q9CYG7|TOM34_MOUSE Mitochondrial import receptor subunit TOM34 OS=Mus mus sp|Q7TMF3|NDUAC_MOUSE NADH dehydrogenase [ubiquinone] 1 alpha subcomplex sub $>$ sp|P12242|UCP1_MOUSE Mitochondrial brown fat uncoupling protein 1 OS=Mus musculus sp|Q8K2B3|DHSA_MOUSE Succinate dehydrogenase [ubiquinone] flavoprotein subunit, $\mathrm{m}$ Sp|Q99LB2|DHRS4_MOUSE Dehydrogenase/reductase SDR family member 4 OS=Mus mu tr|Q924B0|Q924B0_MOUSE Inositol (Myo)-1(Or 4)-monophosphatase 1 OS=Mus musculus sp|Q9Z2I0|LETM1_MOUSE LETM1 and EF-hand domain-containing protein 1, mitochond $>$ sp|Q9CWZ7|SNAG_MOUSE Gamma-soluble NSF attachment protein OS=Mus musculus sp|Q9Z0X1|AIFM1_MOUSE Apoptosis-inducing factor 1, mitochondrial OS=Mus musculus sp|Q9CQQ7|AT5F1_MOUSE ATP synthase subunit b, mitochondrial OS=Mus musculus GN $>$ sp|Q2TPA8|HSDL2_MOUSE Hydroxysteroid dehydrogenase-like protein 2 OS=Mus muscul $>$ sp|Q7TNF8|RIMB1_MOUSE Peripheral-type benzodiazepine receptor-associated protein 1 sp|P17156|HSP72_MOUSE Heat shock-related $70 \mathrm{kDa}$ protein $2 \mathrm{OS}=$ Mus musculus GN=H sp|Q60932|VDAC1_MOUSE Voltage-dependent anion-selective channel protein 1 OS=Mus $>$ sp|Q78PY7|SND1_MOUSE Staphylococcal nuclease domain-containing protein 1 OS=Mus sp|P51881|ADT2 MOUSE ADP/ATP translocase 2 OS=Mus musculus GN=Slc25a5 PE=1 sp|Q91ZR1|RAB4B_MOUSE Ras-related protein Rab-4B OS=Mus musculus GN=Rab4b PE $>$ sp|P56375|ACYP2_MOUSE Acylphosphatase-2 OS=Mus musculus GN=Acyp2 PE=2 SV=2 sp|P19096|FAS_MOUSE Fatty acid synthase OS=Mus musculus GN=Fasn $P E=1 \mathrm{SV}=2$ sp|P46471|PRS7_MOUSE 26S protease regulatory subunit 7 OS=Mus musculus GN=Psmc2 $>$ sp|Q9DCM0|ETHE1_MOUSE Protein ETHE1, mitochondrial OS=Mus musculus GN=Ethe sp|Q921H8|THIKA_MOUSE 3-ketoacyl-CoA thiolase A, peroxisomal OS=Mus musculus G sp|Q9DCN2|NB5R3 MOUSE NADH-cytochrome b5 reductase 3 OS $=$ Mus musculus GN=C: $>$ sp|P15105|GLNA_MOUSE Glutamine synthetase OS=Mus musculus GN=Glul PE $=1 \mathrm{SV}=6$ sp|P06151|LDHA_MOUSE L-lactate dehydrogenase A chain OS=Mus musculus GN=Ldha sp|Q8BX02|KANK2_MOUSE KN motif and ankyrin repeat domain-containing protein $2 \mathrm{OS}$ $>$ sp|Q9D3P8|PLRKT_MOUSE Plasminogen receptor (KT) OS=Mus musculus GN=Plgrkt PE sp|P58281|OPA1_MOUSE Dynamin-like 120 kDa protein, mitochondrial OS=Mus musculus Sp|Q8BTZ7|GMPPB_MOUSE Mannose-1-phosphate guanyltransferase beta OS=Mus musC sp|Q3UIU2|NDUB6_MOUSE NADH dehydrogenase [ubiquinone] 1 beta subcomplex subu sp|O70591|PFD2_MOUSE Prefoldin subunit 2 OS=Mus musculus GN=Pfdn2 PE $=2 \mathrm{SV}=2$ sp|Q9DAR7|DCPS_MOUSE m7GpppX diphosphatase OS=Mus musculus GN=Dcps PE=1 sp|Q9CQW1|YKT6_MOUSE Synaptobrevin homolog YKT6 OS=Mus musculus GN=Ykt6 sp|P63085|MK01_MOUSE Mitogen-activated protein kinase 1 OS=Mus musculus GN=Map $>$ sp|Q8VCW8|ACSF2_MOUSE Acyl-CoA synthetase family member 2, mitochondrial OS=M (N) Q60930|VDAC2_MOUSE Voltage-dependent anion-selective channel protein 2 OS=Mus

sp|Q62167|DDX3X_MOUSE ATP-dependent RNA helicase DDX3X OS=Mus musculus GN 
$>$ sp|E9PZQ0|RYR1_MOUSE Ryanodine receptor 1 OS=Mus musculus GN=Ryr1 PE=1 SV=1 $>$ sp|Q8CI43|MYL6B_MOUSE Myosin light chain 6B OS=Mus musculus GN=Myl6b PE=2 SV=1 $>$ sp|P26883|FKB1A_MOUSE Peptidyl-prolyl cis-trans isomerase FKBP1A OS=Mus musculus GN=Fkb $>$ sp|Q5SX39|MYH4_MOUSE Myosin-4 OS=Mus musculus GN=Myh4 PE=1 SV=1

sp|Q02566|MYH6_MOUSE Myosin-6 OS=Mus musculus GN=Myh6 PE=1 SV=2

SP|Q5SX 40|MYH1_MOUSE Myosin-1 OS=Mus musculus GN=Myh1 PE=1 SV=1
1.944

$1.978-0.162$
$>$ sp|Q99KR3|LACB2_MOUSE Beta-lactamase-like protein 2 OS=Mus musculus GN=Lactb2 sp|Q922B1|MACD1_MOUSE O-acetyl-ADP-ribose deacetylase MACROD1 OS=Mus musc $>$ sp|Q8CAQ8|IMMT_MOUSE Mitochondrial inner membrane protein OS=Mus musculus GN $>$ sp|P52196|THTR_MOUSE Thiosulfate sulfurtransferase OS=Mus musculus GN=Tst PE=1 S sp|P30416|FKBP4_MOUSE Peptidyl-prolyl cis-trans isomerase FKBP4 OS=Mus musculus C sp|Q01768|NDKB_MOUSE Nucleoside diphosphate kinase B OS=Mus musculus GN=Nme $>$ sp|P99028|QCR6_MOUSE Cytochrome b-c1 complex subunit 6, mitochondrial OS=Mus mu sp|Q921G7|ETFD_MOUSE Electron transfer flavoprotein-ubiquinone oxidoreductase, mito $>$ sp|Q8QZT1|THIL_MOUSE Acetyl-CoA acetyltransferase, mitochondrial OS=Mus musculus $>$ sp|Q8K4Z3|NNRE_MOUSE NAD(P)H-hydrate epimerase OS=Mus musculus GN=Apoa1bp $>$ sp|Q9JHW2|NIT2_MOUSE Omega-amidase NIT2 OS=Mus musculus GN=Nit2 PE=1 SV=1 $>$ sp|P05132|KAPCA_MOUSE cAMP-dependent protein kinase catalytic subunit alpha OS=M $>$ sp|Q99LX0|PARK7_MOUSE Protein DJ-1 OS=Mus musculus GN=Park7 PE=1 SV=1

$>$ sp|Q6PHN9|RAB35_MOUSE Ras-related protein Rab-35 OS=Mus musculus GN=Rab35 PE $>\operatorname{tr} \mid$ G5E895|G5E895_MOUSE MCG142264, isoform CRA_b OS=Mus musculus GN=Akr1b1 $>$ sp|P62264|RS14_MOUSE 40S ribosomal protein S14 OS=Mus musculus GN=Rps14 PE=2 $\mathrm{s}$ $>$ sp|P46638|RB11B_MOUSE Ras-related protein Rab-11B OS=Mus musculus GN=Rab11b P $>$ sp|P08228|SODC_MOUSE Superoxide dismutase [Cu-Zn] OS=Mus musculus GN=Sod1 PE $>$ sp|Q9CQA3|DHSB_MOUSE Succinate dehydrogenase [ubiquinone] iron-sulfur subunit, mit $>$ sp|P52503|NDUS6_MOUSE NADH dehydrogenase [ubiquinone] iron-sulfur protein 6, mito $>$ sp|Q8CG76|ARK72_MOUSE Aflatoxin B1 aldehyde reductase member 2 OS=Mus musculu $>$ sp|Q64105|SPRE_MOUSE Sepiapterin reductase OS=Mus musculus GN=Spr $\mathrm{PE}=1 \mathrm{SV}=1$ $>$ sp|Q64516|GLPK_MOUSE Glycerol kinase OS=Mus musculus GN=Gk PE=2 SV=2 $>$ sp|P03930|ATP8_MOUSE ATP synthase protein 8 OS=Mus musculus GN=Mtatp8 PE=1 SV $>$ sp|P28271|ACOC_MOUSE Cytoplasmic aconitate hydratase OS=Mus musculus GN=Aco1 F $>$ sp|P51174|ACADL_MOUSE Long-chain specific acyl-CoA dehydrogenase, mitochondrial O $>$ sp|Q9QX60|DGUOK_MOUSE Deoxyguanosine kinase, mitochondrial OS=Mus musculus G $>$ sp|Q9DBL1|ACDSB_MOUSE Short/branched chain specific acyl-CoA dehydrogenase, mito $>$ sp|Q8BMF3|MAON_MOUSE NADP-dependent malic enzyme, mitochondrial OS=Mus mus $>$ sp|Q9DCT2|NDUS3_MOUSE NADH dehydrogenase [ubiquinone] iron-sulfur protein 3, mi sp|Q8R2Y8|PTH2_MOUSE Peptidyl-tRNA hydrolase 2, mitochondrial OS=Mus musculus $>$ sp|Q9Z1Q9|SYVC_MOUSE Valine--tRNA ligase OS=Mus musculus GN=Vars PE=2 SV=1 $>$ sp|P28474|ADHX_MOUSE Alcohol dehydrogenase class-3 OS=Mus musculus GN=Adh5 P >sp|Q9JLJ2|AL9A1_MOUSE 4-trimethylaminobutyraldehyde dehydrogenase OS=Mus musC $>$ sp|P35385|HSPB7_MOUSE Heat shock protein beta-7 OS=Mus musculus GN=Hspb7 PE=1 $>$ sp|Q8BH04|PCKGM_MOUSE Phosphoenolpyruvate carboxykinase [GTP], mitochondrial O $>$ sp|P37040|NCPR_MOUSE NADPH--cytochrome P450 reductase OS=Mus musculus GN=P $>$ sp|Q9CZB0|C560_MOUSE Succinate dehydrogenase cytochrome b560 subunit, mitochondr >sp|Q9DCS3|MECR_MOUSE Trans-2-enoyl-CoA reductase, mitochondrial OS=Mus muscul $>$ sp|Q6P8J7|KCRS_MOUSE Creatine kinase S-type, mitochondrial OS=Mus musculus $\mathrm{GN}=\mathrm{C}$ Q sp|Q8C3X2CC90_MOUSE Coiled-oil domain-coraning pos $>$ sp|O70378|EMC8_MOUSE ER membrane protein complex subunit 8 OS=Mus musculus GN
$>$ sp|Q922D8|C1TC_MOUSE C-1-tetrahydrofolate synthase, cytoplasmic OS=Mus musculus C $>$ sp|Q9D0S9|HINT2_MOUSE Histidine triad nucleotide-binding protein 2, mitochondrial OS= $>$ sp|Q9CR21|ACPM_MOUSE Acyl carrier protein, mitochondrial OS=Mus musculus GN=Nd $>$ sp|Q3V132|ADT4_MOUSE ADP/ATP translocase 4 OS=Mus musculus GN=Slc25a31 PE=2 Sp|Q9QYB1|CLIC4 MOUSE Chloride intracellular channel protein $4 \mathrm{OS}=\mathrm{Mus}$ musculus $\mathrm{GN}$

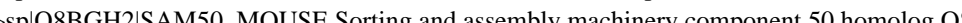
>sp|Q9CQX2|CYB5B_MOUSE Cytochrome b5 type B OS=Mus musculus GN=Cyb5b PE=1 : 
$>$ sp|Q91V92|ACLY_MOUSE ATP-citrate synthase OS=Mus musculus GN=Acly PE $=1 \mathrm{SV}=$ sp|Q9QZ23|NFU1_MOUSE NFU1 iron-sulfur cluster scaffold homolog, mitochondrial OS= sp|Q9R0H0|ACOX1_MOUSE Peroxisomal acyl-coenzyme A oxidase 1 OS=Mus musculus sp|Q8C0V9|FRMD6_MOUSE FERM domain-containing protein 6 OS=Mus musculus GN= $>$ sp|Q06185|ATP5I_MOUSE ATP synthase subunit e, mitochondrial OS=Mus musculus GN= sp|P11352|GPX1_MOUSE Glutathione peroxidase 1 OS=Mus musculus GN=Gpx1 PE=1 S $>$ sp|Q5SWU9|ACACA_MOUSE Acetyl-CoA carboxylase $1 \mathrm{OS}=$ Mus musculus GN=Acaca PI sp|P19157|GSTP1_MOUSE Glutathione S-transferase P 1 OS=Mus musculus GN=Gstp1 PE $>$ sp|P31786|ACBP_MOUSE Acyl-CoA-binding protein OS=Mus musculus GN=Dbi PE=1 S $>$ sp|P47791|GSHR_MOUSE Glutathione reductase, mitochondrial OS=Mus musculus GN=G sp|P06801|MAOX_MOUSE NADP-dependent malic enzyme OS=Mus musculus GN=Me1 $>$ sp|P28867|KPCD_MOUSE Protein kinase C delta type OS=Mus musculus GN=Prkcd PE=1 $>$ sp|Q9CQ75|NDUA2_MOUSE NADH dehydrogenase [ubiquinone] 1 alpha subcomplex sub sp|Q5U458|DJC11_MOUSE DnaJ homolog subfamily C member 11 OS=Mus musculus GN sp|Q9JK48|SHLB1_MOUSE Endophilin-B1 OS=Mus musculus GN=Sh3glb1 PE=1 SV=1 $>$ sp|Q9R112|SQRD_MOUSE Sulfide:quinone oxidoreductase, mitochondrial OS=Mus muscu sp|Q99MN9|PCCB_MOUSE Propionyl-CoA carboxylase beta chain, mitochondrial OS=Mus $>$ sp|Q8BIJ6|SYIM_MOUSE Isoleucine--tRNA ligase, mitochondrial OS=Mus musculus GN=1 sp|Q91WS0|CISD1_MOUSE CDGSH iron-sulfur domain-containing protein 1 OS=Mus mus sp|Q99LC3|NDUAA_MOUSE NADH dehydrogenase [ubiquinone] 1 alpha subcomplex sub tr|A2AFQ2|A2AFQ2_MOUSE 3-hydroxyacyl-CoA dehydrogenase type-2 OS=Mus muscul $>$ sp|Q8K1R7|NEK9_MOUSE Serine/threonine-protein kinase Nek9 OS=Mus musculus GN= sp|Q8BH59|CMC1_MOUSE Calcium-binding mitochondrial carrier protein Aralar1 OS=M $>$ sp|Q99MN1|SYK_MOUSE Lysine--tRNA ligase OS=Mus musculus GN=Kars PE=1 SV=1 $>$ sp|Q9Z1Z2|STRAP_MOUSE Serine-threonine kinase receptor-associated protein OS=Mus $m$ tr|Q5XJF6|Q5XJF6_MOUSE Ribosomal protein OS=Mus musculus GN=Rpl10a PE=2 SV=1 $>$ sp|Q99JB2|STML2_MOUSE Stomatin-like protein 2 OS=Mus musculus GN=Stoml2 PE=1 sp|O35129|PHB2_MOUSE Prohibitin-2 OS=Mus musculus GN=Phb2 PE=1 SV=1

tr|Q91VA7|Q91VA7_MOUSE Isocitrate dehydrogenase 3 (NAD+) beta OS=Mus musculus sp|Q8R4NO|CLYBL_MOUSE Citrate lyase subunit beta-like protein, mitochondrial OS=M sp|P62259|1433E_MOUSE 14-3-3 protein epsilon OS=Mus musculus GN=Ywhae PE=1 SV = $>$ sp|Q920E5|FPPS_MOUSE Farnesyl pyrophosphate synthase OS=Mus musculus GN=Fdps $P$ $>$ sp|Q9WV85|NDK3_MOUSE Nucleoside diphosphate kinase 3 OS=Mus musculus GN=Nme Sp|E9Q557|DESP_MOUSE Desmoplakin OS=Mus musculus $\mathrm{GN}=\mathrm{Dsp} \mathrm{PE}=3 \mathrm{SV}=1$

$>$ sp|Q91WK5|GCSH_MOUSE Glycine cleavage system H protein, mitochondrial OS=Mus mı 

$>$ sp|P05202|AATM_MOUSE Aspartate aminotransferase, mitochondrial OS=Mus musculus $>$ sp|Q9QUIO|RHOA_MOUSE Transforming protein RhoA OS=Mus musculus GN=Rhoa PE= sp|P47738|ALDH2_MOUSE Aldehyde dehydrogenase, mitochondrial OS=Mus musculus Gr $>$ sp|O55137|ACOT1_MOUSE Acyl-coenzyme A thioesterase 1 OS=Mus musculus GN=Aco sp|O70571|PDK4_MOUSE [Pyruvate sp|Q60936|ADCK3 MOUSE Chaperone activi

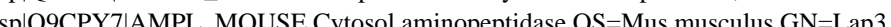
$>$ sp|Q9D6K8|FUND2_MOUSE FUN14 domain-containing protein 2 OS=Mus musculus GN= sp|Q9CPV4|GLOD4_MOUSE Glyoxalase domain-containing protein 4 OS=Mus musculus sp|O35459|ECH1_MOUSE Delta(3,5)-Delta(2,4)-dienoyl-CoA isomerase, mitochondrial OS: sp|P61922|GABT_MOUSE 4-aminobutyrate aminotransferase, mitochondrial OS=Mus mus $>$ sp|Q9Z2Z6|MCAT_MOUSE Mitochondrial carnitine/acylcarnitine carrier protein OS=Mus $>$ sp|O35855|BCAT2 MOUSE Branched-chain-amino-acid aminotransferase, mitochondrial O $>$ sp|Q9JIQ3|DBLOH_MOUSE Diablo homolog, mitochondrial OS=Mus musculus GN=Diab $>$ sp|Q8CHT0|AL4A1_MOUSE Delta-1-pyrroline-5-carboxylate dehydrogenase, mitochondri $>$ sp|Q99L13|3HIDH_MOUSE 3-hydroxyisobutyrate dehydrogenase, mitochondrial OS=Mus sp|P45952|ACADM_MOUSE Medium-chain specific acyl-CoA dehydrogenase, mitochondri sp|P03911|NU4M_MOUSE NADH-ubiquinone oxidoreductase chain 4 OS=Mus musculus sp|Q3TL44|NLRX1_MOUSE NLR family member X1 OS=Mus musculus GN=Nlrx1 PE=2 $>\mathrm{sp}|\mathrm{P} 18242|$ CATD MOUSE Cathepsin D OS=Mus musculus GN=Ctsd PE $=1 \mathrm{SV}=1$

sp|P84091|AP2M1_MOUSE AP-2 complex subunit mu OS=Mus musculus GN=Ap2m1 PE sp|Q99J99|THTM_MOUSE 3-mercaptopyruvate sulfurtransferase OS=Mus musculus GN=M $>$ sp|P80313|TCPH_MOUSE T-complex protein 1 subunit eta OS=Mus musculus GN=Cct7 PE $>$ sp|Q9CPQ3|TOM22_MOUSE Mitochondrial import receptor subunit TOM22 homolog OS= sp|Q922W5|P5CR1_MOUSE Pyrroline-5-carboxylate reductase 1, mitochondrial OS=Mus m $>$ sp|Q9DCX2|ATP5H_MOUSE ATP synthase subunit d, mitochondrial OS=Mus musculus G $>\mathrm{sp}|\mathrm{Q} 68 \mathrm{FD} 5| \mathrm{CLH} 1$ MOUSE Clathrin heavy chain $1 \mathrm{OS}=$ Mus musculus $\mathrm{GN}=\mathrm{Cltc} \mathrm{PE}=1 \mathrm{SV}=$ sp|P10605|CATB_MOUSE Cathepsin B OS=Mus musculus GN=Ctsb PE $=1 \mathrm{SV}=2$

$>$ pp|P70404|IDHG1_MOUSE Isocitrate dehydrogenase [NAD] subunit gamma 1, mitochondri sp|P16332|MUTA_MOUSE Methylmalonyl-CoA mutase, mitochondrial OS=Mus musculus sp|P50136|ODBA_MOUSE 2-oxoisovalerate dehydrogenase subunit alpha, mitochondrial Os $>$ pp|Q8C5H8|NAKD1_MOUSE NAD kinase domain-containing protein $1 \mathrm{OS}=$ Mus musculus >sp|P48962|ADT1_MOUSE ADP/ATP translocase 1 OS=Mus musculus GN=Slc25a4 PE=1 S $>$ sp|Q9CZD3|SYG_MOUSE Glycine--tRNA ligase OS=Mus musculus GN=Gars PE=1 SV=1 sp|Q9CQ69|QCR8_MOUSE Cytochrome b-c1 complex subunit 8 OS=Mus musculus GN=U sp|O88844|IDHC_MOUSE Isocitrate dehydrogenase [NADP] cytoplasmic OS=Mus muscul sp|Q9R0X4|ACOT9_MOUSE Acyl-coenzyme A thioesterase 9, mitochondrial OS=Mus mus sp|Q9CR62|M2OM_MOUSE Mitochondrial 2-oxoglutarate/malate carrier protein OS=Mus tr|E9QPQ8|E9QPQ8 MOUSE 39S ribosomal protein L48, mitochondrial OS=Mus musculus $>$ sp|O08749|DLDH_MOUSE Dihydrolipoyl dehydrogenase, mitochondrial OS=Mus musculus $>$ sp|Q99LD8|DDAH2_MOUSE N(G),N(G)-dimethylarginine dimethylaminohydrolase $2 \mathrm{OS}=$ sp|Q9D6Y7|MSRA_MOUSE Mitochondrial peptide methionine sulfoxide reductase OS=M $>$ sp|Q63844|MK03_MOUSE Mitogen-activated protein kinase 3 OS=Mus musculus GN=Ma sp|Q99LB6|MAT2B MOUSE Methionine adenosyltransferase 2 subunit beta OS=Mus mus $>$ sp|P52825|CPT2_MOUSE Carnitine O-palmitoyltransferase 2, mitochondrial OS=Mus mus sp|P39688|FYN_MOUSE Tyrosine-protein kinase Fyn OS=Mus musculus GN=Fyn PE=1 S >sp|Q9CWJ9|PUR9_MOUSE Bifunctional purine biosynthesis protein PURH OS=Mus muscu 

$>$ sp|Q99KB8|GLO2_MOUSE Hydroxyacylglutathione hydrolase, mitochondrial OS=Mus mu $>$ sp|P12787|COX5A_MOUSE Cytochrome c oxidase subunit 5A, mitochondrial OS=Mus mus $>$ sp|P41216|ACSL1_MOUSE Long-chain-fatty-acid--CoA ligase 1 OS=Mus musculus GN=A sp|Q9CZ42|NNRD_MOUSE ATP-dependent (S)-NAD(P)H-hydrate dehydratase OS=Mus m sp|Q8R164|BPHL_MOUSE Valacyclovir hydrolase OS=Mus musculus GN=Bphl PE=2 SV= $>$ sp|Q9JK42|PDK2_MOUSE [Pyruvate dehydrogenase [lipoamide]] kinase isozyme 2, mitoch $>$ sp|Q3ULD5|MCCB_MOUSE Methylcrotonoyl-CoA carboxylase beta chain, mitochondrial C $>$ sp|Q91VS7|MGST1_MOUSE Microsomal glutathione S-transferase 1 OS=Mus musculus G $>$ sp|Q9D019|SYRC_MOUSE Arginine--tRNA ligase, cytoplasmic OS=Mus musculus GN=R sp|Q9QUM9|PSA6_MOUSE Proteasome subunit alpha type-6 OS=Mus musculus GN=Psma sp|P70296|PEBP1_MOUSE Phosphatidylethanolamine-binding protein 1 OS=Mus musculus $>$ sp|Q8BFR5|EFTU_MOUSE Elongation factor Tu, mitochondrial OS=Mus musculus GN=Tu $>$ pp|P00329|ADH1_MOUSE Alcohol dehydrogenase $1 \mathrm{OS}=$ Mus musculus $\mathrm{GN}=\mathrm{Adh} 1 \mathrm{PE}=2 \mathrm{~S}$ sp|Q9D023|MPC2_MOUSE Mitochondrial pyruvate carrier 2 OS=Mus musculus GN=Mpc2 sp|P16125|LDHB_MOUSE L-lactate dehydrogenase B chain OS=Mus musculus GN=Ldhb $>$ sp|Q9D0M3|CY1_MOUSE Cytochrome c1, heme protein, mitochondrial OS=Mus musculus $>$ sp|Q61425|HCDH_MOUSE Hydroxyacyl-coenzyme A dehydrogenase, mitochondrial OS=M $>$ sp|Q8K1J6|TRNT1_MOUSE CCA tRNA nucleotidyltransferase 1, mitochondrial OS=Mus sp|Q9QYA2|TOM40_MOUSE Mitochondrial import receptor subunit TOM40 homolog OS $>$ sp|Q32MW3|ACO10_MOUSE Acyl-coenzyme A thioesterase 10, mitochondrial OS=Mus m $>$ sp|P97742|CPT1A_MOUSE Carnitine O-palmitoyltransferase 1, liver isoform OS=Mus mus $>$ sp|Q8BH95|ECHM_MOUSE Enoyl-CoA hydratase, mitochondrial OS=Mus musculus GN= $>$ sp|P08249|MDHM_MOUSE Malate dehydrogenase, mitochondrial OS=Mus musculus GN= sp $\mid$ O08528|HXK2_MOUSE Hexokinase-2 OS=Mus musculus GN=Hk2 PE=1 SV=1

sp|Q9JLZ3|AUHM_MOUSE Methylglutaconyl-CoA hydratase, mitochondrial OS=Mus mu sp|P29758|OAT_MOUSE Ornithine aminotransferase, mitochondrial OS=Mus musculus GN sp|P20108|PRDX3_MOUSE Thioredoxin-dependent peroxide reductase, mitochondrial OS= $>$ sp|Q9CQN6|TM14C_MOUSE Transmembrane protein 14C OS=Mus musculus GN=Tmem $>$ sp|Q91WD5|NDUS2_MOUSE NADH dehydrogenase [ubiquinone] iron-sulfur protein 2, mi $>$ sp|Q07813|BAX_MOUSE Apoptosis regulator BAX OS=Mus musculus GN=Bax PE=1 SV= sp|Q8JZN5|ACAD9 MOUSE Acyl-CoA dehydrogenase family member 9, mitochondrial Os sp|Q9QYR9|ACOT2_MOUSE Acyl-coenzyme A thioesterase 2, mitochondrial OS=Mus mu $>$ sp|P09671|SODM_MOUSE Superoxide dismutase [Mn], mitochondrial OS=Mus musculus $>$ pp|Q05920|PYC_MOUSE Pyruvate carboxylase, mitochondrial OS=Mus musculus GN=Pc sp|P63101|1433Z_MOUSE 14-3-3 protein zeta/delta OS=Mus musculus GN=Ywhaz PE=1 sp|Q91VR2|ATPG_MOUSE ATP synthase subunit gamma, mitochondrial OS=Mus musculu >sp|P99029|PRDX5 _MOUSE Peroxiredoxin-5, mitochondrial OS=Mus musculus GN=Prdx5 sp|P58044|IDI1_MOUSE Isopentenyl-diphosphate Delta-isomerase 1 OS=Mus musculus GN sp|Q6PB66|LPPRC_MOUSE Leucine-rich PPR motif-containing protein, mitochondrial OS= sp|P67778|PHB_MOUSE Prohibitin OS=Mus musculus GN=Phb PE=1 SV=1

sp|Q9D1G1|RAB1B_MOUSE Ras-related protein Rab-1B OS=Mus musculus GN=Rab1b P $>$ pp|Q9CRD0|OCAD1_MOUSE OCIA domain-containing protein $1 \mathrm{OS}=$ Mus musculus GN= $>$ sp|P47934|CACP_MOUSE Carnitine O-acetyltransferase OS=Mus musculus GN=Crat PE=1 $>\mathrm{sp} \mid \mathrm{P} 18572$ |BASI_MOUSE Basigin OS=Mus musculus GN=Bsg $\mathrm{PE}=1 \mathrm{SV}=2$

sp|Q99JY0|ECHB_MOUSE Trifunctional enzyme subunit beta, mitochondrial OS=Mus mus 
$>$ sp|Q91W97|HKDC1_MOUSE Putative hexokinase HKDC1 OS=Mus musculus GN=Hkdc1 sp|Q8BWT1|THIM_MOUSE 3-ketoacyl-CoA thiolase, mitochondrial OS=Mus musculus G $>$ sp|P54310|LIPS_MOUSE Hormone-sensitive lipase OS=Mus musculus GN=Lipe PE=1 SV sp|Q8JZQ2|AFG32_MOUSE AFG3-like protein 2 OS=Mus musculus GN=Afg312 PE=1 SV $>$ sp|Q99L04|DHRS1_MOUSE Dehydrogenase/reductase SDR family member 1 OS=Mus mus $>$ sp|Q99LB7|SARDH_MOUSE Sarcosine dehydrogenase, mitochondrial OS=Mus musculus C sp|P54071|[DHP MOUSE Isocitrate dehydrogenase [NADP], mitochondrial OS=Mus musc sp|P56480|ATPB_MOUSE ATP synthase subunit beta, mitochondrial OS=Mus musculus G sp|Q99KI0|ACON_MOUSE Aconitate hydratase, mitochondrial OS=Mus musculus GN=Ac $>$ sp|Q9DC70|NDUS7_MOUSE NADH dehydrogenase [ubiquinone] iron-sulfur protein 7, mit sp|Q9WUR2|ECI2_MOUSE Enoyl-CoA delta isomerase 2, mitochondrial OS=Mus musculu $>$ pp|Q9DCB8|ISCA2_MOUSE Iron-sulfur cluster assembly 2 homolog, mitochondrial $O S=M$ P $>$ pp|P11499|HS90B_MOUSE Heat shock protein HSP 90-beta OS=Mus musculus GN=Hsp90 sp|Q9CQ54|NDUC2_MOUSE NADH dehydrogenase [ubiquinone] 1 subunit C2 OS=Mus m $>$ sp|P35235|PTN11_MOUSE Tyrosine-protein phosphatase non-receptor type 11 OS=Mus m >sp|Q99MR8|MCCA_MOUSE Methylcrotonoyl-CoA carboxylase subunit alpha, mitochondri $>$ sp|P56135|ATPK_MOUSE ATP synthase subunit $\mathrm{f}$, mitochondrial OS=Mus musculus GN= $>$ sp|Q9JHR7|IDE_MOUSE Insulin-degrading enzyme OS=Mus musculus GN=Ide PE=1 SV= sp|Q9CXZ1|NDUS4_MOUSE NADH dehydrogenase [ubiquinone] iron-sulfur protein 4, mi sp|Q91ZA3|PCCA_MOUSE Propionyl-CoA carboxylase alpha chain, mitochondrial OS=M sp|Q04447|KCRB_MOUSE Creatine kinase B-type OS=Mus musculus GN=Ckb PE=1 SV=1 sp|Q8BMP6|GCP60_MOUSE Golgi resident protein GCP60 OS=Mus musculus GN=Acbd3 $>$ sp|P63328|PP2BA_MOUSE Serine/threonine-protein phosphatase 2B catalytic subunit alph $>$ sp|Q03265|ATPA_MOUSE ATP synthase subunit alpha, mitochondrial OS=Mus musculus sp|Q9CZU6|CISY MOUSE Citrate synthase, mitochondrial OS=Mus musculus GN=Cs PE= $>$ sp|P24270|CATA_MOUSE Catalase OS=Mus musculus GN=Cat $P E=1 \mathrm{SV}=4$

$>$ sp|Q91VD9|NDUS1_MOUSE NADH-ubiquinone oxidoreductase $75 \mathrm{kDa}$ subunit, mitochon $>$ sp|Q8CGK3|LONM_MOUSE Lon protease homolog, mitochondrial OS=Mus musculus GN sp|P59017|B2L13_MOUSE Bcl-2-like protein 13 OS=Mus musculus GN=Bcl2113 PE=1 SV sp|Q9CQZ5|NDUA6_MOUSE NADH dehydrogenase [ubiquinone] 1 alpha subcomplex sub $>$ sp|Q64373|B2CL1_MOUSE Bcl-2-like protein 1 OS=Mus musculus GN=Bcl2l1 PE=1 SV=1 $>$ sp|Q9JHI5|IVD_MOUSE Isovaleryl-CoA dehydrogenase, mitochondrial OS=Mus musculus $>$ sp|O08709|PRDX6 MOUSE Peroxiredoxin-6 OS=Mus musculus GN=Prdx6 PE=1 SV=3

$>$ sp|Q8VEM8|MPCP_MOUSE Phosphate carrier protein, mitochondrial OS=Mus musculus G] sp|Q9D3D9|ATPD_MOUSE ATP synthase subunit delta, mitochondrial OS=Mus musculus sp|P38647|GRP75_MOUSE Stress-70 protein, mitochondrial OS=Mus musculus GN=Hspa9 sp|Q9EQ20|MMSA_MOUSE Methylmalonate-semialdehyde dehydrogenase [acylating], mi $>$ sp|Q60597|ODO1_MOUSE 2-oxoglutarate dehydrogenase, mitochondrial OS=Mus muscul us $>$ sp|P54775|PRS6B_MOUSE 26S protease regulatory subunit 6B OS=Mus musculus GN=Psn >sp|Q9DB77|QCR2_MOUSE Cytochrome b-c1 complex subunit 2, mitochondrial OS=Mus m $>$ sp|P26638|SYSC _MOUSE Serine--tRNA ligase, cytoplasmic OS=Mus musculus GN=Sars P sp|Q9WTP6|KAD2_MOUSE Adenylate kinase 2, mitochondrial OS=Mus musculus GN=Ak sp|Q9CZ13|QCR1_MOUSE Cytochrome b-c1 complex subunit 1, mitochondrial OS=Mus $>$ sp|Q9CPQ8|ATP5L_MOUSE ATP synthase subunit g, mitochondrial OS=Mus musculus GN $>$ sp|Q62465|VAT1_MOUSE Synaptic vesicle membrane protein VAT-1 homolog OS=Mus m $>$ sp|Q9DB20|ATPO MOUSE ATP synthase subunit $\mathrm{O}$, mitochondrial OS=Mus musculus $\mathrm{GN}$ 
$>$ sp|Q91YT0|NDUV1_MOUSE NADH dehydrogenase [ubiquinone] flavoprotein 1, mitochon $>$ sp|Q9D855|QCR7_MOUSE Cytochrome b-c1 complex subunit 7 OS=Mus musculus GN=U $>$ sp|P07901|HS90A_MOUSE Heat shock protein HSP 90-alpha OS=Mus musculus GN=Hsp9 sp|P31938|MP2K1_MOUSE Dual specificity mitogen-activated protein kinase kinase 1 OS=1 $>$ sp|O54724|PTRF MOUSE Polymerase I and transcript release factor OS=Mus musculus GN $>$ sp|P63168|DYL1_MOUSE Dynein light chain 1, cytoplasmic OS=Mus musculus GN=Dynll $>$ sp|Q9CYH2|F213A_MOUSE Redox-regulatory protein FAM213A OS=Mus musculus GN=I $>$ sp|O08553|DPYL2_MOUSE Dihydropyrimidinase-related protein 2 OS=Mus musculus GN sp|Q64442|DHSO_MOUSE Sorbitol dehydrogenase OS=Mus musculus GN=Sord PE=1 SV $>$ sp|P54822|PUR8_MOUSE Adenylosuccinate lyase OS=Mus musculus GN=Adsl PE=2 SV=، $>$ sp|Q9D051|ODPB_MOUSE Pyruvate dehydrogenase E1 component subunit beta, mitochon $>$ sp|Q9DCW4|ETFB_MOUSE Electron transfer flavoprotein subunit beta OS=Mus musculus (1) (1) ent subunit alpha, somatic $\mathrm{f}$ sp|Q9ERS2|NDUAD_MOUSE NADH dehydrogenase [ubiquinone] 1 alpha subcomplex sub $>$ sp|O89023|TPP1_MOUSE Tripeptidyl-peptidase 1 OS=Mus musculus GN=Tpp1 PE=1 SV=: sp|P63038|CH60_MOUSE $60 \mathrm{kDa}$ heat shock protein, mitochondrial OS=Mus musculus GN $>$ sp|Q9CQR4|ACO13_MOUSE Acyl-coenzyme A thioesterase 13 OS=Mus musculus GN=A $>$ sp|Q64433|CH10 MOUSE $10 \mathrm{kDa}$ heat shock protein, mitochondrial OS=Mus musculus $\mathrm{GN}$ (1) sp|P19536_COX5B_LOUSE Cytochome c oxidase subuit 5B, mitochndia OS=Mus mus sp|Q9CQ92|FIS1_MOUSE Mitochondrial fission 1 protein OS=Mus musculus GN=Fis1 PE sp|Q9D2G2|ODO2_MOUSE Dihydrolipoyllysine-residue succinyltransferase component of sp|P62492|RB11A_MOUSE Ras-related protein Rab-11A OS=Mus musculus GN=Rab11a $>$ sp|Q9CQH3|NDUB5_MOUSE NADH dehydrogenase [ubiquinone] 1 beta subcomplex subu tr $|\mathrm{Q} 3 \mathrm{UKH} 3| \mathrm{Q} 3 \mathrm{UKH} 3$ MOUSE Putative uncharacterized protein $\mathrm{OS}=\mathrm{Mus}$ musculus $\mathrm{GN}=\mathrm{Ac}$ sp|Q8QZS1|MIBCH_MOUSE 3-hydroxyisobutyyl-CoA hydrolase, sp|P85094|SC2A_MOUSE Isochorismatase domain-containing protein 2A, nitochondrial sp|Q9D6U8|F162A_MOUSE Protein FAM162A OS=Mus musculus GN=Fam162a PE=2 S $>$ sp|Q8BMF4|ODP2_MOUSE Dihydrolipoyllysine-residue acetyltransferase component of py $>$ pp|Q9CWSO|DDAH1_MOUSE N(G),N(G)-dimethylarginine dimethylaminohydrolase 1 OS sp|P17710|HXK1_MOUSE Hexokinase-1 OS=Mus musculus GN=Hk1 PE=1 SV=3

sp|P97807|FUMH_MOUSE Fumarate hydratase, mitochondrial OS=Mus musculus GN=Fh P $>$ sp|P00405|COX_MOUSE Cytochrome c oxidase subunit 2 OS=Mus musculus GN=Mtco2 
$>$ sp|O08599|STXB1_MOUSE Syntaxin-binding protein $1 \mathrm{OS}=$ Mus musculus GN=Stxbp1 PE=

sp|Q05421|CP2E1_MOUSE Cytochrome P450 2E1 OS=Mus musculus GN=Cyp2e1 PE=1 S

sp|P00397|COX1_MOUSE Cytochrome c oxidase subunit 1 OS=Mus musculus GN=Mtco1

sp|Q07417|ACADS_MOUSE Short-chain specific acyl-CoA dehydrogenase, mitochondrial

sp|Q62425|NDUA4_MOUSE NADH dehydrogenase [ubiquinone] 1 alpha subcomplex sub

sp|Q8R1I1|QCR9_MOUSE Cytochrome b-c1 complex subunit 9 OS=Mus musculus GN=Uq

sp|Q9DC69|NDUA9_MOUSE NADH dehydrogenase [ubiquinone] 1 alpha subcomplex subu

sp|Q9JMH6|TRXR1_MOUSE Thioredoxin reductase 1, cytoplasmic OS=Mus musculus GN-

Pp|P50518|VATE1_MOUSE V-type poton ATPase 1, cybit E 1 OS=Mus musculus GN=A

sp|Q64133|AOFA_MOUSE Amine oxidase [flavin-containing] A OS=Mus musclus GN=M

$>$ sp|Q8VCL2|SCO2_MOUSE Protein SCO2 homolog, mitochondrial OS=Mus musculus GN=

tr|D3YZ32|D3YZ32_MOUSE Mitochondrial fission 1 protein OS=Mus musculus GN=Fis1

tr|Q80X68|Q80X68_MOUSE Citrate synthase OS=Mus musculus GN=Csl PE=1 SV=1

sp|A2ARZ3|FSIP2_MOUSE Fibrous sheath-interacting protein $2 \mathrm{OS}=$ Mus musculus GN=F

tr|Q3TCI7|Q3TCI7_MOUSE Putative uncharacterized protein OS=Mus musculus GN=Ldh 